\title{
The Unfolded Protein Response and its interplay with the MAPK-mediated pheromone response pathway in Ustilago maydis
}

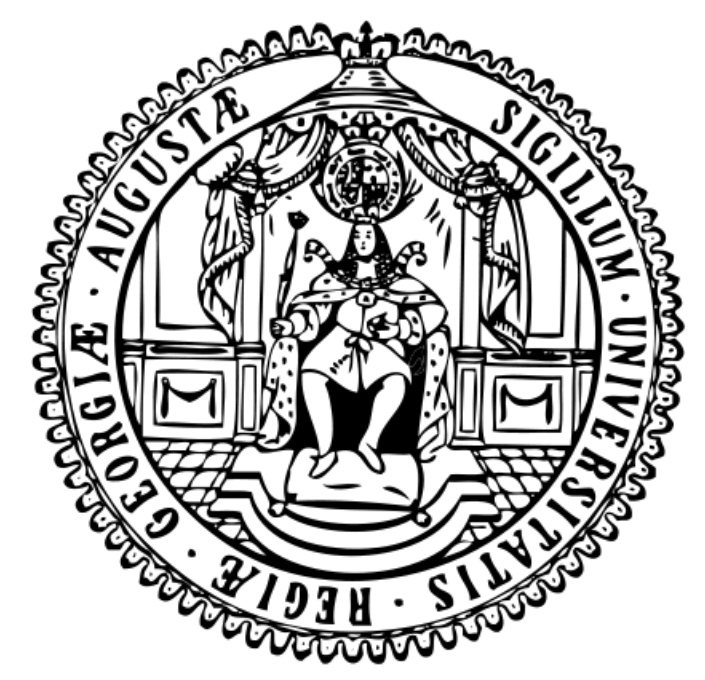

\section{Dissertation}

for the award of the degree

"Doctor rerum naturalium"

\author{
of the Georg-August-Universität Göttingen \\ within the doctoral program "PRoTECT (IRTG2172)" \\ of the Georg-August-University School of Science (GAUSS) \\ submitted by \\ Lara Schmitz \\ from Gummersbach
}

Göttingen 2019 



\section{Thesis Committee}

apl. Prof. Dr. Kai Heimel (Supervisor and $1^{\text {st }}$ Reviewer)

Institute of Microbiology and Genetics

Department of Molecular Microbiology and Genetics, Georg-August-University Göttingen

Prof. Dr. Gerhard Braus (2 ${ }^{\text {nd }}$ Reviewer)

Institute of Microbiology and Genetics

Department of Molecular Microbiology and Genetics, Georg-August-University Göttingen

Prof. Dr. James Kronstad

Michael Smith Laboratories

Department of Microbiology and Immunology

Faculty of Agricultural Sciences, The University of British Columbia

\section{Members of the Examination Board}

Prof. Dr. Ivo Feußner

Albrecht von Haller Institute

Department of Plant Biochemistry, Georg-August-University Göttingen

Prof. Dr. Volker Lipka

Schwann-Schleiden Research Center

Department of Plant Cell Biology, Georg-August-University Göttingen

Prof. Dr. Andrea Polle

Büsgen Institute

Department of Forest Botany and Tree Physiology, Georg-August-University Göttingen

Date of oral examination: 11.07.2019 


\section{List of publications}

\section{Publications part of this dissertation:}

Schmitz, L., McCotter, S., Kretschmer, M., Kronstad, J.W., and Heimel, K. (2018) Transcripts and tumors: regulatory and metabolic programming during biotrophic phytopathogenesis. F1000 Res 7: 1-8.

Schmitz, L., Schwier, M.A., Heimel, K. (2019) Suppression of mating-type signaling via the unfolded protein response is mediated by the dual specificity phosphatase Rok1. (unpublished)

Schmitz, L., Kronstad, J.W., Heimel, K. (2019) Novel tools for conditional gene expression enable the analysis of stage-specific protein functions in the Ustilago maydis/maize pathosystem. (unpublished)

\section{Other publications:}

Hampel, M., Jakobi, M., Schmitz, L., Meyer, U., Finkernagel, F., Doehlemann, G., and Heimel, K. (2016) Unfolded Protein Response (UPR) Regulator Cib1 Controls Expression of Genes Encoding Secreted Virulence Factors in Ustilago maydis. PLoS One 11: 1-16. 


\section{Table of Content}

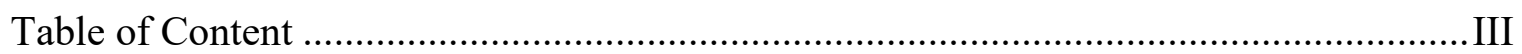

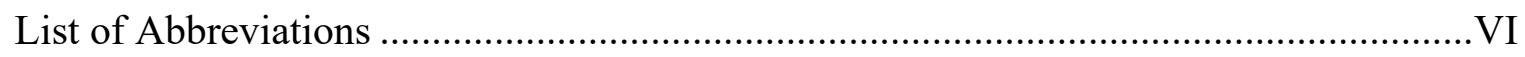

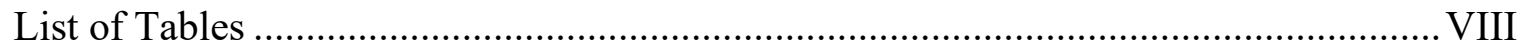

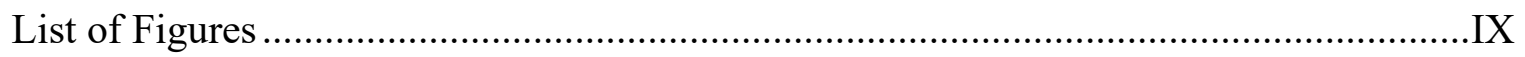

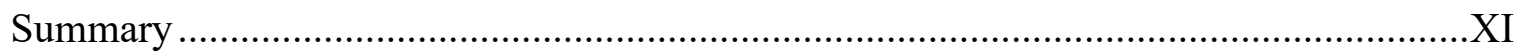

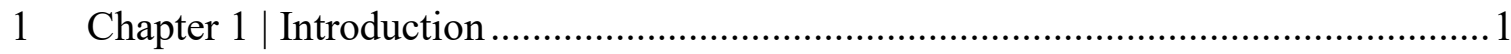

$1.1 \quad$ The Unfolded Protein Response....................................................................

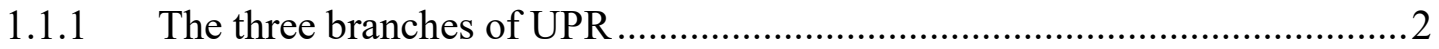

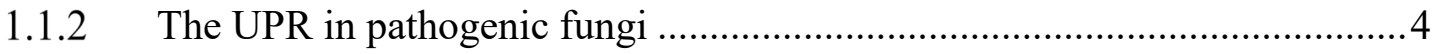

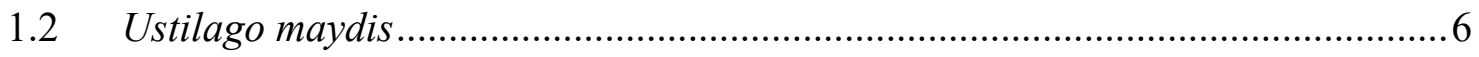

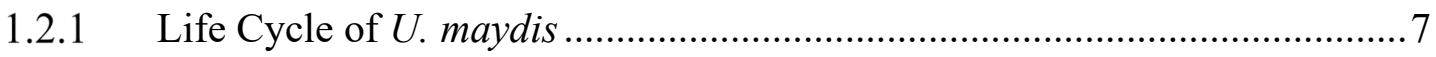

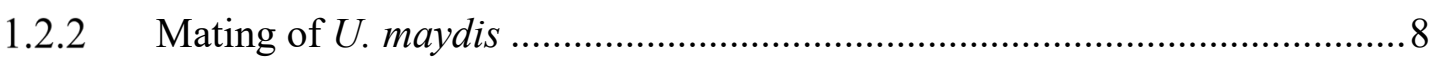

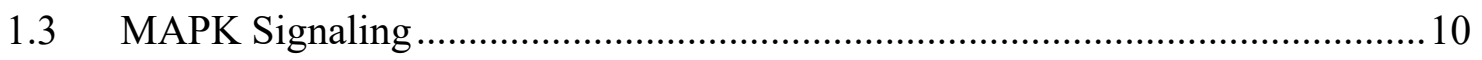

1.3.1 The pheromone response in U. maydis is mediated by MAPK- and

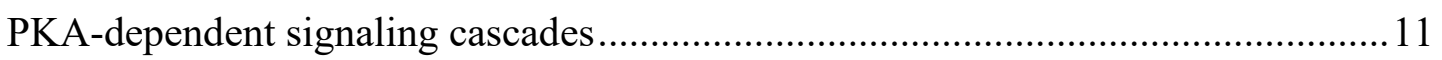

1.3.2 The dual specificity phosphatase Rok1 inhibits the pheromone response...13

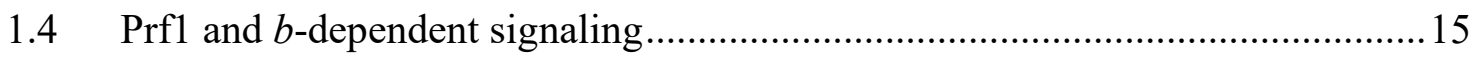

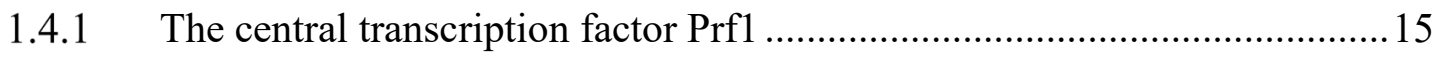

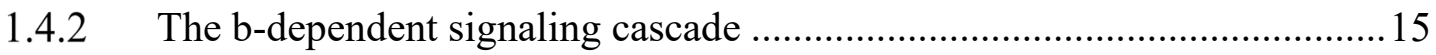

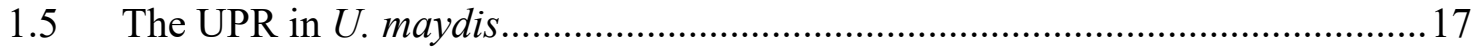

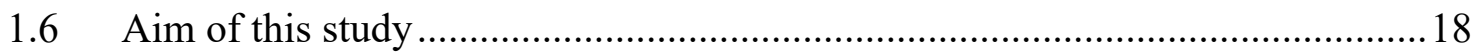

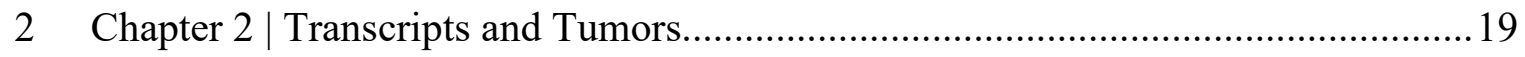

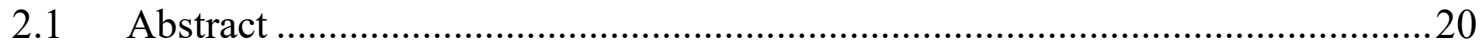

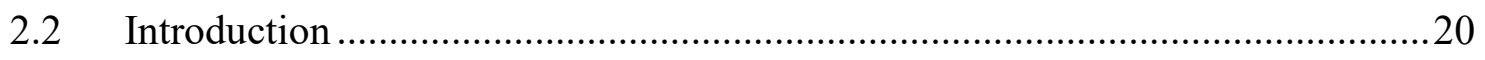

2.3 Transcriptome analysis throughout the infection process..............................22

2.4 Transcriptome changes reveal extensive metabolic remodeling in tumors.........24 
Table of Content

2.5 Beyond U. maydis: transcriptome studies with other smut fungi .....................26

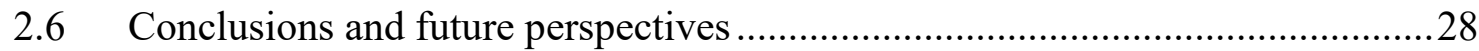

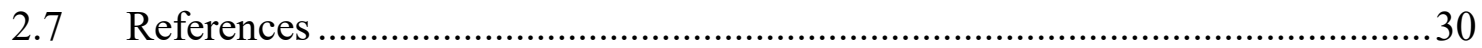

3 Chapter 3 | UPR-dependent inhibition of mating type signaling...............................35

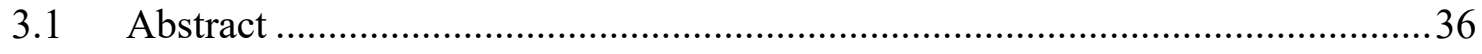

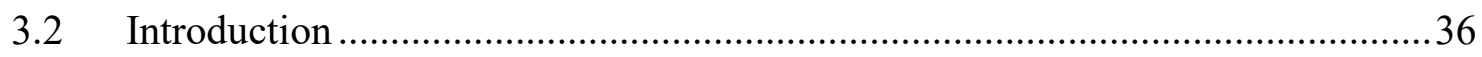

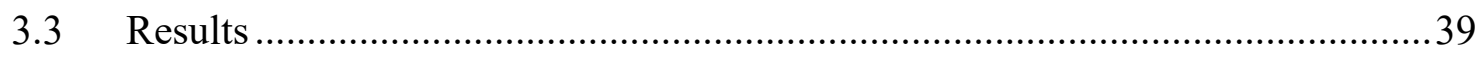

3.3.1 UPR activity inhibits $b$-mating type dependent regulatory network ...........39

3.3.2 UPR represses morphological and transcriptional response to pheromone.41

3.3.3 Induced expression of $f u z 7^{D D}$ rescues the morphological but not the transcriptional UPR-dependent inhibition of pheromone signaling ..........................43

3.3.4 Phosphorylation of Kpp2 is suppressed by the UPR ...............................45

3.3.5 UPR-dependent suppression of Kpp2 phosphorylation depends on the dual

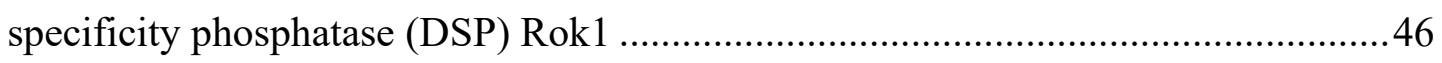

3.3.6 The UPR affects mating type-dependent signaling upstream and downstream of $\mathrm{bE} / \mathrm{bW}$

3.3.7 The MAPK Kpp2 is dispensable for biotrophic development in planta .....51

3.4 Discussion 53

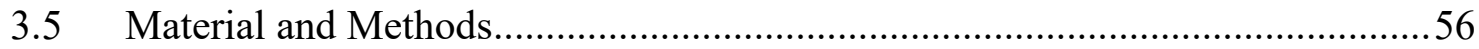

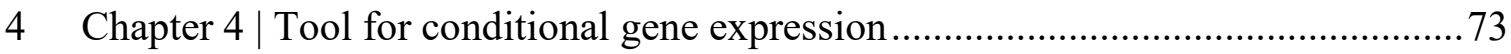

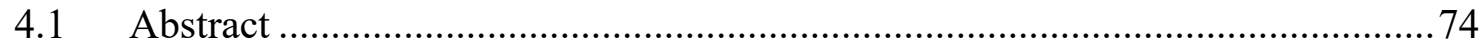

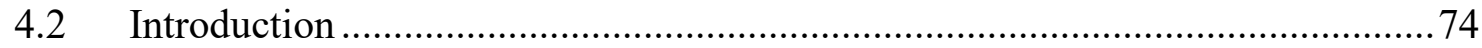

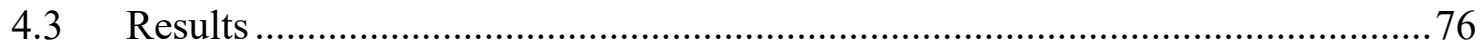

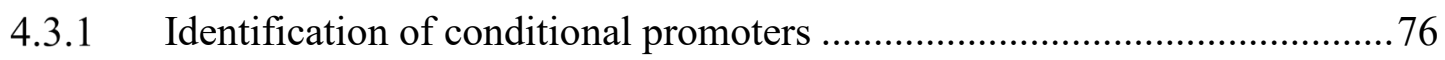

4.3.2 Cib1 is required throughout biotrophic development in planta ...................78

4.3.3 Genomic localization and presence of a resistance cassette affects gene

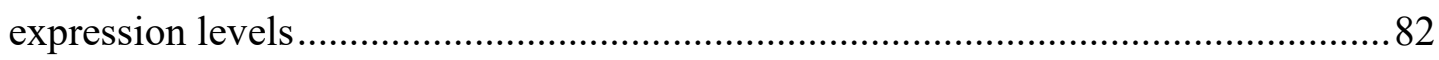


4.3.4 Overexpression of $c i b 1^{\text {s }}$ does not disturb pathogenic development in planta 85

4.4 Discussion .88

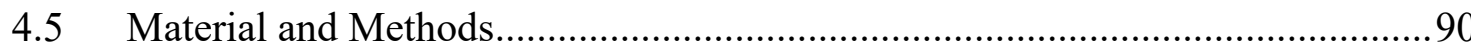

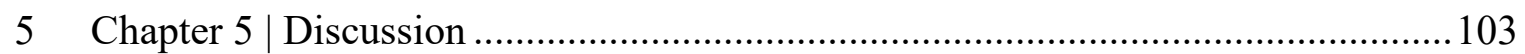

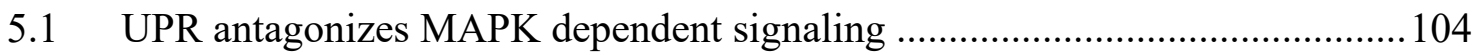

5.1.1 Active UPR signaling interferes with $a$ - and $b$-mating type dependent gene expression and $b$-dependent filament formation 104

5.1.2 UPR-dependent dephosphorylation of the MAPK Kpp2 represses the morphological and transcriptional response to a pheromone stimulus. 105

5.1.3 UPR-dependent suppression of Kpp2 phosphorylation depends on the DSP Rok1 107

5.1.4 The UPR and mating type-dependent signaling cascades interact on multiple levels 110

5.1.5 Role of the Kpp2-Rok1 interaction during pathogenic development in planta 110

5.2 A new approach for conditional gene expression

5.2.1 Promoters with conditional activity enable stage-specific gene expression 113

5.2.2 The locus of gene integration and the presence of resistance cassettes affect gene expression

5.2.3 UPR activity is important at later stages of biotrophic development in planta 116

5.2.4 Overexpression of $c i b 1^{\mathrm{s}}$ in planta does not lead to UPR hyperactivation.118

References 120 


\section{List of Abbreviations}

\begin{tabular}{|c|c|c|c|}
\hline$\%$ & percent & Hyg & hygromycin \\
\hline$\Delta$ & deletion & $\operatorname{Hyg}^{\mathrm{R}}$ & hygromycin resistance \\
\hline$\mu \mathrm{g}$ & microgram & Kan & kanamycin \\
\hline$\mu \mathrm{l}$ & microliter & $\mathrm{Kan}^{\mathrm{R}}$ & kanamycin resistance \\
\hline$\mu \mathrm{m}$ & micrometer & $\mathrm{kb}$ & kilobase \\
\hline Ade & adenine & $\mathrm{kDa}$ & kilo Dalton $=1000$ Dalton \\
\hline Amp & ampicillin & 1 & liter \\
\hline $\mathrm{Amp}^{\mathrm{R}}$ & ampicillin resistance & $\mathrm{M}$ & molar \\
\hline approx. & approximately & MAPK & mitogen-activated protein \\
\hline Ara & arabinose & & kinase \\
\hline ATP & adenosine triphosphate & MAPKK & MAPK-kinase \\
\hline bp & base pair & MAPKKK & MAPKK-kinase \\
\hline${ }^{\circ} \mathrm{C}$ & degree Celsius & $\mathrm{mg}$ & milligram \\
\hline ca. & circa & $\min$ & minute \\
\hline \multirow[t]{2}{*}{ cAMP } & Cyclic adenosine & $\mathrm{mL}$ & milliliter \\
\hline & monophosphate & $\mathrm{mM}$ & millimolar \\
\hline $\mathrm{Cbx}$ & carboxine & mRNA & messenger RNA \\
\hline $\mathrm{CC}$ & charcoal & $\mathrm{NaCl}$ & sodium chloride \\
\hline $\mathrm{CM}$ & complete medium & $\mathrm{Nat}^{\mathrm{R}}$ & Nourseothricin resistance \\
\hline C-terminal & carboxy-terminal & $\mathrm{nm}$ & nanometer \\
\hline DMSO & dimethyl sulfoxide & N-terminal & amino-terminal \\
\hline DNA & deoxyribonucleic acid & $\mathrm{OD}_{600}$ & optical density at 600 \\
\hline \multirow[t]{2}{*}{ dNTP } & deoxyribonucleoside & & nanometer \\
\hline & triphosphate & ORF & open reading frame \\
\hline DTT & dithiothreitol & PCR & polymerase chain reaction \\
\hline E. coli & Escherichia coli & PD & potato dextrose \\
\hline e.g. & exempli gratia & pers. & Personal \\
\hline et al. & and others & $P f u$ & Pyrococcus furiosus \\
\hline $\mathrm{EtOH}$ & ethanol & PKA & protein kinase $\mathrm{A}$ \\
\hline Glc & glucose & $\mathrm{pH}$ & potentia hydrogenii \\
\hline h & hour & RNA & ribonucleic acid \\
\hline $\mathrm{H}_{2} \mathrm{O}$ & hydrogen oxide & RT & room temperature \\
\hline
\end{tabular}


S. cerevisiae Saccharamyces cerevisiae

U. maydis Ustilago maydis

WT

wild type
YEPS yeast extract, peptone,

sucrose 
List of Tables

\section{List of Tables}

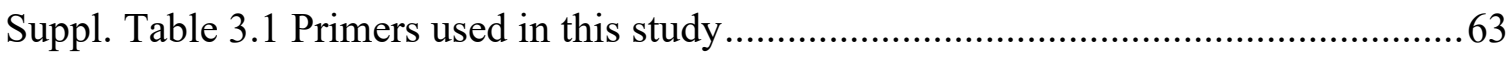

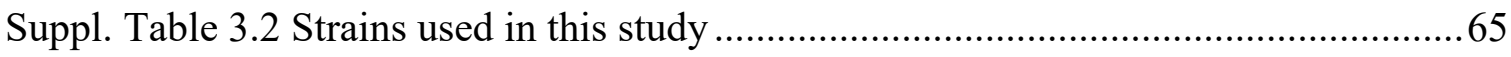

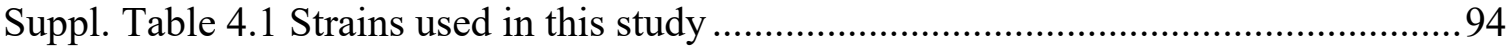

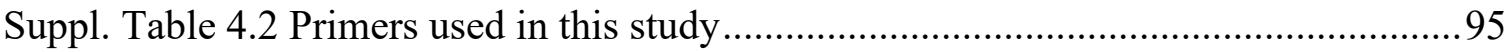




\section{List of Figures}

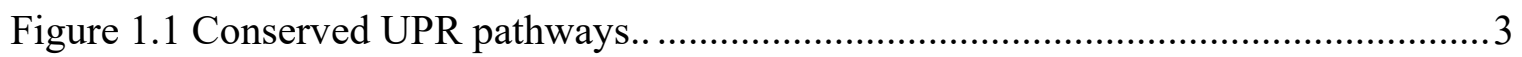

Figure 1.2 Corn smut disease caused by $U$. maydis .................................................. 6

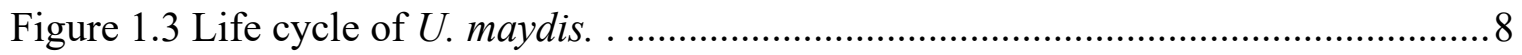

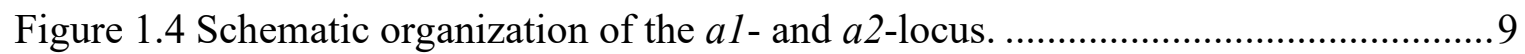

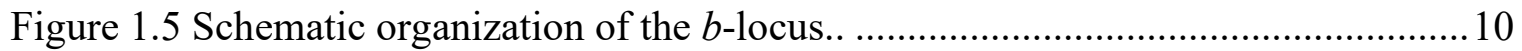

Figure 1.6 Schematic picture of the pheromone dependent MAPK signaling cascade.....12

Figure 1.7 Prf1- and bE/bW-dependent regulation of initial stages of pathogenic

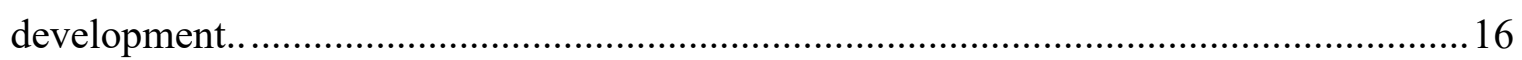

Figure 2.1 Tumor formation on maize by $U$. maydis ....................................................2

Figure 2.2 Regulators, effectors, and metabolism during Ustilago maydis infection of maize.

Figure 3.1 UPR suppresses b-dependent filament formation and gene expression. (A). ..40 Figure 3.2 The morphological and transcriptional response to pheromone is inhibited by UPR activity

Figure 3.3 The transcriptional and morphological response bifurcates downstream of Fuz7..

Figure 3.4 Expression of $c i b 1^{s}$ strongly reduces Kpp2 phosphorylation

Figure 3.5 Dephosphorylation of Kpp2 upon $c i b 1^{s}$ expression depends on the DPS Rok1..

Figure 3.6 Constitutive expression of $b E / b W$ does not restore filamentous growth or gene expression.. .50

Figure $3.7 \mathrm{Kpp} 2$ is dispensable for pathogenic development in planta............................52

Figure 4.1 Identification and testing of promoters for conditional gene expression.........77

Figure 4.2 Conditional cibl expression restores ER-stress resistance, but not pathogenicity..

Figure 4.3 Conditional cibl expression impairs pathogenic development and induces $P R$ gene expression in planta. .81

Figure 4.4 The locus of integration and presence of a resistance cassette influence gene expression.. .85

Figure 4.5 Expression of cib1 is required to coordinate $c i b 1^{s}$ activity to enable full virulence of $U$. maydis.. .88 
List of Figures

Figure 5.1 Model of the crosstalk between the UPR and mating type signaling pathways..

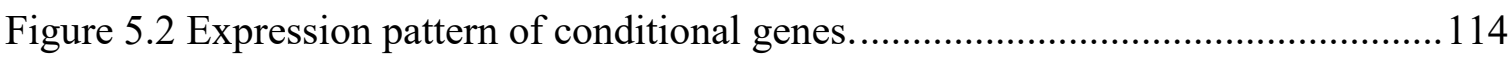

Figure 5.3 Cloning strategies for conditional gene expression................................... 116

\section{List of Supplemental Figures}

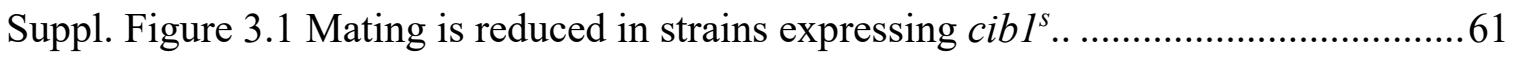

Suppl. Figure 3.2 Rok1 does physically interact with the MAPKs Kpp2 and Kpp6........62

Suppl. Figure 3.3 rokl deletion does not restore expression of the $a$-mating type genes and prf1. 62

Suppl. Figure 4.1 $\triangle U M A G_{-} 12184$ and $\triangle U M A G_{-} 03597$ strains do not show increased sensitivity towards cell wall- or ER-stress. .94 


\section{Summary}

Ustilago maydis is a biotrophic plant pathogen that causes the corn smut disease. Sexual and pathogenic development of the fungus are tightly connected, U. maydis relies on its host, Zea mays, to complete its life cycle. Prerequisite for plant infection is the fusion of two compatible, haploid sporidia to form the infectious dikaryotic filament. Cell/cell recognition and the subsequent fusion event are regulated by the biallelic $a$-mating type locus. Perception of compatible pheromone triggers a G2 cell cycle arrest and conjugation tube formation. After formation of the infectious filament, the $\mathrm{bE} / \mathrm{bW}$-heterodimer, encoded by the multiallelic $b$-mating type locus, controls all further steps of pathogenic development, including maintenance of the G2 cell cycle arrest, filamentous growth and formation of appressoria to penetrate the plant surface.

After plant penetration, U. maydis relies on the unfolded protein response (UPR), a conserved eukaryotic signaling pathway, to ensure efficient secretion of effectors to suppress the plant defense response and establish a compatible biotrophic interaction. The UPR is specifically activated in planta and continuously active during plant infection. However, premature UPR activity interferes with formation of the dikaryotic filament by inhibiting $b E, b W$ and $r b f l$ expression.

In this study, I show that the UPR affects pathogenic development on multiple levels. UPR activity not only has extensive inhibitory effects on the $b$-dependent transcription factor network, but also interferes with the transcriptional and morphological response to pheromone. UPR activity leads to increased dephosphorylation of the MAPK Kpp2 by the dual specificity phosphatase Rok1, leading to reduced activity of the pheromone response factor 1 (Prf1), and the Prf1-regulated $a$ - and $b$-mating type genes. Crosstalk between the UPR and mating type pathways provide a regulatory feedback mechanism to dampen the $a$ - and $b$-mating type pathways. In this way, hypervirulence is prevented and the compatible biotrophic fungal/plant interaction is preserved. Since effector gene expression is regulated by the $b$-pathway, increased UPR activity results in reduced effector gene expression, thereby effectively lowering endoplasmic reticulum (ER) stress levels.

Moreover, I established a novel expression system, allowing the depletion or overexpression of any given gene at defined stages of biotrophic development in planta. Proof-of-principle experiments demonstrated that a functional UPR is not only required directly after plant penetration, but also crucial to ensure continuous suppression of plant defense responses at later stages of pathogenic development in planta. 
Summary

In summary, I uncovered novel regulatory cross-connections between pathways regulating the ER stress response and pathogenic growth of the fungus in planta. My results strongly suggest that efficient host colonization and maintenance of fungal biotrophy are balanced by negative feedback loops between pathways, aligning the intracellular physiology to the organismic interaction between the fungus and its host plant. 


\section{Chapter $1 \mid$ Introduction}

\subsection{The Unfolded Protein Response}

The ER is the largest organelle in eukaryotic cells and a dynamic network. Synthesis, folding, modification and quality control of proteins take place at/in the ER (reviewed in Braakman and Hebert, 2013). After translation initiation in the cytosol, the ribosome-mRNA complex is recruited to the ER membrane by the signal recognition particle (SRP) and the protein is co-translationally inserted into the ER. During and after protein synthesis, secreted and transmembrane proteins are folded and further modified. Two of the most common modifications are N-glycosylation and the formation of disulfide bonds (Siberstein and Gilmore, 1996; Fassio and Sitia, 2002). These modifications can potentially affect stability, conformation/folding and function of a protein (Helenius, 2002; Hanson et al., 2009; Wallis and Freedman, 2011). To most secretory proteins, N-linked glycans are added by an oligosaccharyltransferase. This modification takes place during or soon after translocation of the polypeptide into the ER (Nilsson and von Heijne, 1993). Protein disulfide isomerases catalyze the formation, isomerization and reduction of disulfide bonds, thereby also assisting the correct folding of a protein (Braakman and Hebert, 2013). Correctly folded and modified proteins are targeted to the Golgi apparatus and are subsequently secreted or transported to the cell membrane. During protein folding and modification, truncated polypeptides, misfolded intermediates and non-natively modified proteins can form aggregates and accumulate in the cell (Dobson, 2003; Hartl and Hayer-Hartl, 2009). Acutely damaged proteins are either repaired or targeted for degradation by the ER-Associated protein Degradation (ERAD) machinery (Smith et al., 2011; Ruggiano et al., 2014). An imbalance (ER stress) between protein demand of a cell and the capacity of the ER machinery induces an adaptive pathway called Unfolded Protein Response (UPR) (Ron and Walter, 2007). The UPR is a highly conserved eukaryotic signaling pathway to restore ER homeostasis. UPR activity inhibits the overall protein translation, increases expression of chaperones, folding enzymes, ERAD proteins and membrane lipids, the latter resulting in ER expansion (Schuck et al., 2009). Thereby, ER stress is relieved (Cox et al., 1997; Travers et al., 2000). If ER proteostasis cannot be restored, apoptosis is induced to protect surrounding cells from toxic effects (Shore et al., 2011; Tabas and Ron, 2011; Hetz, 2012; Sano and Reed, 2013). 
Chapter 1 | Introduction

\subsubsection{The three branches of UPR}

In mammals/higher eukaryotes, three parallel UPR pathways exist. Each pathway defines a distinct arm of the UPR and consists of a stress transducer integral to the ER membrane that senses un- or misfolded proteins in the ER lumen. The Activating Transcription Factor-6 (ATF6) and the Protein Kinase RNA-like ER Kinase (PERK) pathways are only found in metazoans. By contrast, the Inositol-Requiring Enzyme-1 (IRE1) induced pathway is conserved in all eukaryotes. ATF6 belongs to a class of ER stress transducers localized to the ER membrane, and contains a stress-sensing domain in the ER lumen (Haze et al., 1999) (Figure 1.1A). Upon ER stress, ATF6 is transported to the Golgi apparatus where it is cleaved by Site 1 Protease (S1P) and Site 2 Protease (S2P). Cleavage by these proteases releases a cytosolic DNA-binding portion, $\operatorname{ATF} 6(\mathrm{~N})$. This fragment functions as a Basic Leucin Zipper (bZIP) transcription factor that enters the nucleus and induces expression of UPR target genes (Haze et al., 1999).

The second stress-responsive factor, PERK, is also ER membrane localized (Figure 1.1B). It consists of a cytoplasmic protein kinase domain and a lumenal domain for sensing of ER stress. Upon ER stress, PERK oligomerizes and is activated by trans-autophosphorylation (Bertolotti et al., 2000). Phosphorylated PERK inhibits the $\alpha$-subunit of eukaryotic translation Initiation Factor-2 (eIF2 $\alpha$ ) by phosphorylation. This globally reduces the amount of newly synthesized proteins by lowering the levels of translation initiation (Harding et al., 1999). Interestingly, phosphorylation of eIF2 $\alpha$ also contributes to expression of UPR targets and translation of Activating Transcription Factor 4 (ATF4). ATF4 induces expression of factors involved in amino acid import, glutathione biosynthesis and resistance to oxidative stress (Harding et al., 2003). Among the transcriptionally activated genes is C/EBP-Homologous Protein $(\mathrm{CHOP})$, a factor involved in growth arrest and apoptosis (Zinszner et al., 1998; Ma et al., 2002).

The third branch consists of the evolutionary conserved kinase/RNase IRE1 (Ron and Walter, 2007) (Figure 1.1C). Ire1p was first identified in a screen for mutants defective in KAR2 (BiP in mammals) induction in Saccharomyces cerevisiae (Cox et al., 1993). Similar to PERK, Ire1p also consists of a stress sensor domain in the ER lumen and a cytoplasmic protein kinase domain (Cox et al., 1993; Mori et al., 1993; Harding et al., 1999; Bertolotti et al., 2000). Upon ER stress, binding of un- or misfolded proteins to the lumenal domain of Ire1 $\mathrm{p}$ and dissociation of Kar2p/BiP result in oligomerization and activation of Ire $1 \mathrm{p}$ via trans-autophosphorylation (Sidrauski and Walter, 1997; Korennykh et al., 2009; Walter and Gardner, 2011). In contrast to PERK, Irelp exclusively phosphorylates itself and 
contains an RNase domain that causes unconventional, cytoplasmic splicing of an mRNA encoding a bZIP transcription factor (Shamu and Walter, 1996; Sidrauski and Walter, 1997). This factor is termed Homologous for ATF/CREB 1 (Hac1p) in S. cerevisiae/ X-Box Binding Protein1 (XBP1) in mammals (Cox and Walter, 1996; Kawahara et al., 1998; Rüegsegger et al., 2001; Yoshida et al., 2001). The spliced mRNA is translated into the active transcription factor Hac1p/XBP1, which functions as central regulator of the UPR by binding to Unfolded Protein Response Elements (UPREs) in target gene promoters (Mori et al., 1992). This induces expression of chaperones, components of the ERAD pathway and proteins mediating lipid synthesis resulting in relieve of ER stress (Cox et al., 1997; Travers et al., 2000; Murray et al., 2004).

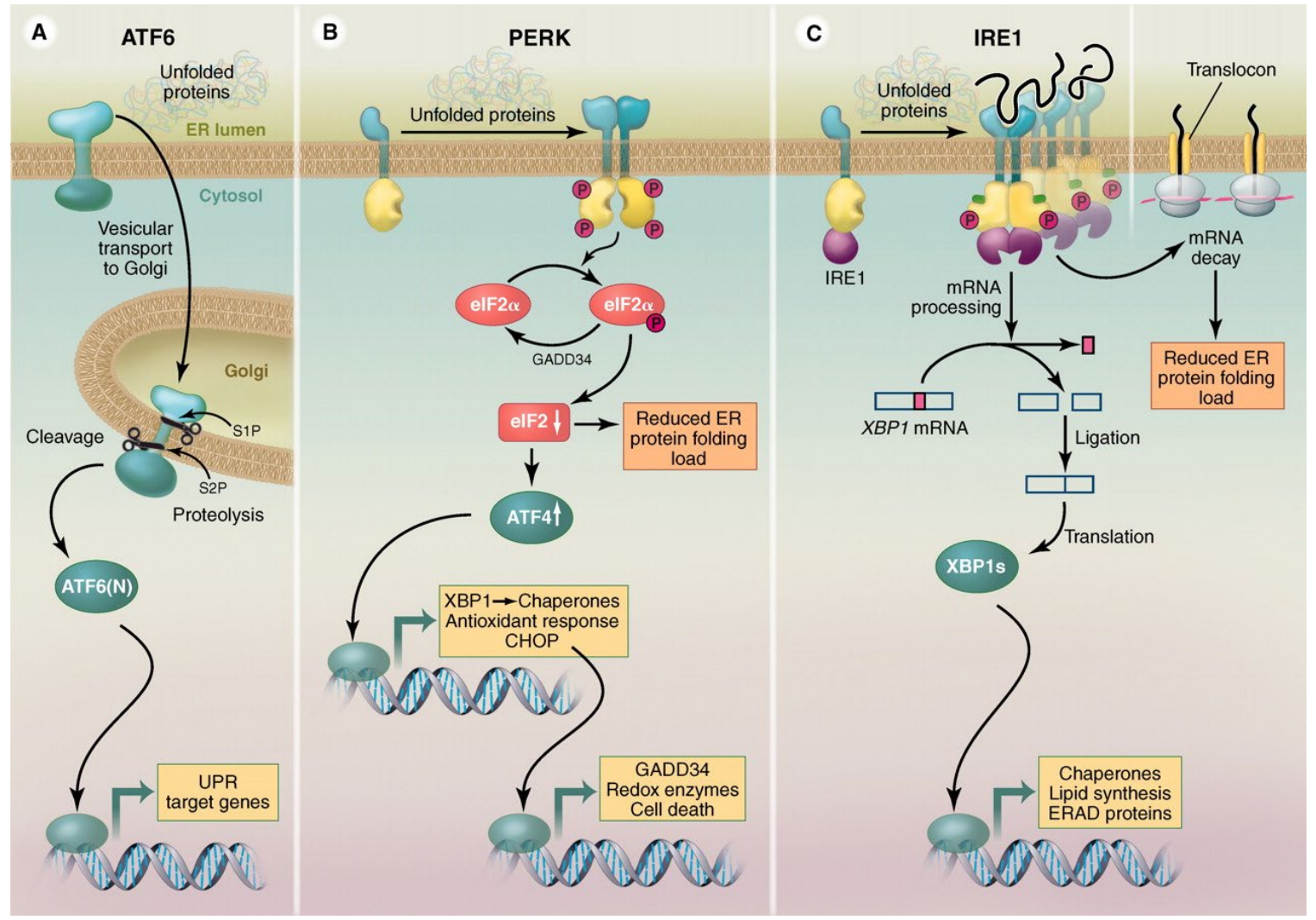

Figure 1.1 Conserved UPR pathways. The three signal transducers (ATF6, PERK, IRE1) sense unfolded proteins in the ER lumen and transmit that information resulting in production of bZIP transcription factors that induce expression of UPR target genes. (A) The ATF6 pathway is only found in metazoans and is activated by proteolysis of ATF6. (B) The PERK pathway is also only found in metazoans and activated by phosphorylation of eIF2 $\alpha$. Thereby, translation of the ATF4 transcription factor is induced. (C) The IRE1 pathway is highly conserved in all eukaryotes. UPR is activated by unconventional cytoplasmic splicing of the mRNA encoding the central UPR transcription factor XBP1/HAC1. Modified from Ron and Walter, 2011. 
In $S$. cerevisiae, the unspliced HAC1 mRNA is present in the cytoplasm during phases of low ER stress. Two layers of post-transcriptional silencing ensure that no unspliced Haclp is present and undesirably activates the UPR (Di Santo et al., 2016). Formation of a secondary structure by the 5' UTR blocks translation initiation by preventing binding of the ribosome (Rüegsegger et al., 2001). Additionally, unspliced Hac1p is rapidly degraded by recognition of the unique $\mathrm{C}$-terminus, ubiquitination and proteasomal degradation. Both layer rely on sequences found in the intron of the mRNA/protein (Di Santo et al., 2016). In mammals, both, the spliced and the unspliced form of XBP1 mRNA are translated but differ in their properties. The protein XBP $1^{\mathrm{u}}$ functions as a negative regulator of the UPR. It contains a nuclear exclusion signal in its $\mathrm{C}$-terminal, $\mathrm{XBP} 1^{\mathrm{u}}$-specific region, enabling the protein to shuttle between nucleus and cytoplasm, while $\mathrm{XBP} 1^{\mathrm{s}}$ is exclusively localized in the nucleus (Tirosh et al., 2006; Yoshida et al., 2006). XBP $1^{\mathrm{u}}$ and $\mathrm{XBP} 1^{\mathrm{s}}$ form a complex in the nucleus which is exported to the cytoplasm and rapidly degraded by the proteasome due to the degradation motif present in XBP1 ${ }^{u}$ (Tirosh et al., 2006; Yoshida et al., 2006; Yoshida et al., 2009).

\subsubsection{The UPR in pathogenic fungi}

The UPR is described as a conserved pathway and central regulator of virulence in human and plant pathogenic fungi (Heimel, 2015). A functional UPR is necessary for interaction with the host and adaption to changing environments during the infection. For the human pathogens Aspergillus fumigatus and Cryptococcus neoformans, being able to grow at body temperature of the host is essential for virulence. Deletion of HAC1 or IRE1 homologs lead to reduced thermotolerance (Richie et al., 2009; Cheon et al., 2011), indicating a conserved connection between the UPR and temperature-dependent growth. Additionally, loss of the UPR increased cell wall stress sensitivity in both fungi (Richie et al., 2009; Cheon et al., 2011), demonstrating interplay between the cell wall integrity (CWI) pathway and the UPR. In S. cerevisiae, the UPR is activated by the CWI MAPK pathway during cell wall stress, suggesting that the connection between cell wall integrity and the UPR might be conserved (Scrimale et al., 2009).

In the necrotrophic pathogen Alternaria brassicicola, the UPR is also important for pathogenic development and adaption to the host (Joubert et al., 2011). Deletion mutants of the gene encoding the Hac1 homolog showed a complete loss of virulence, higher sensitivity towards cell wall stress and reduced secretory capacity (Joubert et al., 2011). 
Additionally, besides ER-stress, the plant-derived compound camalexin is also able to activate the UPR in A. brassicicola, and UPR defective mutants showed higher sensitivity towards camalexin (Joubert et al., 2011). Camalexin most likely damages fungal membranes. In response, the fungus reacts with drug efflux and cell wall reinforcement (Sellam et al., 2007). The rice blast fungus Magnaporte oryzae also depends on the UPR for conidiation and virulence (Tang et al., 2015).

While basic features of the Ire1-induced UPR pathway are conserved in most fungi, the fission yeast Schizosaccharomyces pombe and the human pathogen Candida glabrata constitute exceptions (Kimmig et al., 2012; Miyazaki et al., 2013). S. pombe does not contain a Haclp homolog in its genome, and the ER-stress response solely depends on the homolog of Ire1p. Upon ER-stress, a subset of mRNAs encoding proteins of the secretory pathway are targeted for a process called Regulated Ire1-dependent decay (RIDD), which is normally only found in higher eukaryotes. RIDD is characterized by Ire1-dependent cleavage of various ER-associated mRNAs reducing the total load for the ER folding machinery (Kimmig et al., 2012). Additionally, Ire1 processes Bipl mRNA resulting in its stabilization. In C. glabrata, Ire1 no longer splices Hac1 mRNA, but employs a pathway similar to RIDD to cope with ER stress (Miyazaki et al., 2013).

The UPR in higher eukaryotes is not only important to counteract ER-stress, but is also implicated in a number of developmental processes in organisms like Drosophila, fish, mice and also humans. (reviewed in Mitra and Ryoo, 2019). For fungi, less is known about the role of the UPR in development. In S. cerevisiae, it was shown that activation of the UPR or expression of the spliced Haclp transcription factor suppressed pseudohyphal growth and meiosis (Schröder et al., 2000). Splicing of HACl occurred under high nitrogen conditions, probably due to high protein translation rates and thus, higher amounts of unfolded proteins in the ER (Schröder et al., 2000). In the basidiomycete U. maydis, multiple connections between the UPR and (pathogenic) development have been demonstrated and will be further discussed in 1.5. 


\subsection{Ustilago maydis}

The corn smut pathogen $U$. maydis belongs to the phylum of Basidiomycetes, which constitute together with the Ascomycetes the subkingdom of higher fungi. U. maydis was first described/mentioned by the French biologists Bonnet, Aymen and Tillet around 1750 (Christensen, 1963).

As most smut fungi, U. maydis has a narrow host range and specifically infects corn (Zea mays) and its close relative teosinte (Euchlena mexicana) (Doebley, 1992). In order to successfully infect its host plant, U. maydis has to form a dikaryotic, infectious filament by

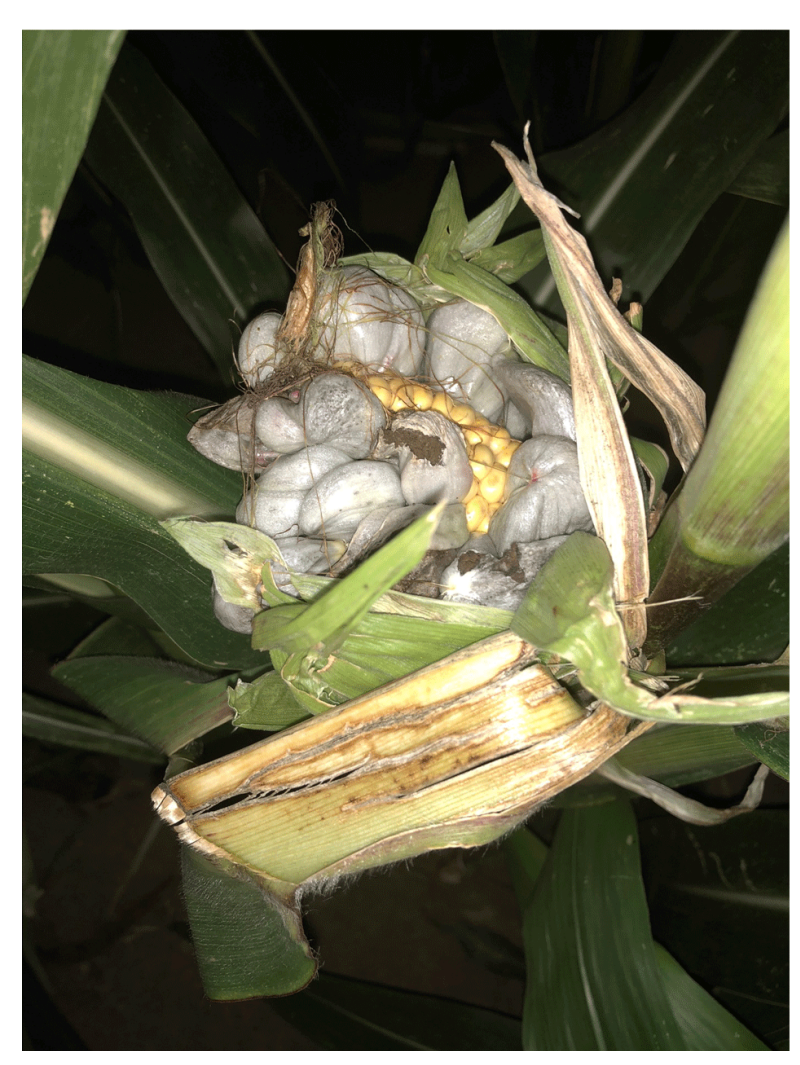

Figure 1.2 Corn smut disease caused by $U$. maydis. Corncob infected by $U$. maydis. Photos were taken in September 2018, Göttingen. From Schmitz et al., 2018 mating with a compatible partner (Banuett and Herskowitz, 2006). Infection with U. maydis induces plant tumor formation (Christensen, 1963) (Figure 1.2). In the tumors, massive fungal proliferation and spore formation take place. At the end of a vegetative period, the diploid, melanin containing spores are released into the environment resulting in the typical "smutted" appearance of infected plants.

U. maydis is an important organism in laboratory research. It is a well-established model organism to analyze basic recombination mechanisms as well as sexual development and fungal-plant interaction (Bakkeren et al., 2008; Steinberg and Perez-Martin, 2008; Brefort et al., 2009a; Lanver et al., 2018).

U. maydis is easy to cultivate in the laboratory, has a fully annotated genome and a variety of established molecular techniques for genetic modification (Brachmann et al., 2004; Feldbrügge et al., 2004; Kämper et al., 2006; Steinberg and Perez-Martin, 2008). Finally, $U$. maydis completes its life cycle within two weeks under laboratory conditions, thus, it constitutes a well-suited model organism to study host-pathogen interactions. These properties establish $U$. maydis as an ideal model organism that provides advantage over other smut and related rust fungi that are often genetically difficult to access. 


\subsubsection{Life Cycle of $\boldsymbol{U}$. maydis}

In U. maydis, pathogenic and sexual development is closely interlinked. Host infection is necessary for proliferation, formation of spores and completion of its life cycle (Banuett, 1992).

The life cycle of $U$. maydis can be divided in a saprophytic and a biotrophic phase. During vegetative growth, the haploid cells of $U$. maydis grow yeast-like by budding (Figure 1.3.1) and are not able to infect the host plant. The onset of the biotrophic stage is initiated upon fusion of two haploid cells and generation of an infectious dikaryotic filament (Banuett, 1992). Sexual and pathogenic development are controlled by a tetrapolar mating type system (Hartmann et al., 1996a; Bölker, 2001; Feldbrügge et al., 2004). The biallelic $a$-mating type locus encodes a pheromone-receptor system which mediates cell/cell recognition and fusion of two genetically compatible cells (Schulz et al., 1990; Bölker et al., 1992). Only cells that differ in their mating type loci are able to fuse and form the infectious hyphae. Perception of compatible pheromone triggers a G2 cell cycle arrest and results in formation of conjugation tubes (Figure 1.3.2) to mediate cell fusion (Figure 1.3.3) (Spellig et al., 1994). After formation of the dikaryotic filament, different alleles of the multiallelic $b$-locus are necessary to maintain the cell cycle arrest, induce filamentous growth and infect the plant (Gillissen et al., 1992). On the leaf surface, dikaryotic filaments elongate by tip-growth with cytoplasm accumulating at the tip of the hyphae while older parts are sealed off by septa (Banuett and Herskowitz, 1994). Plant invasion is mediated by formation of appressoria (Snetselaar and Mims, 1992; Snetselaar and Mims, 1993) (Figure 1.3.4), specific infection structures that facilitate plant penetration independent of turgorbased mechanical force (Figure 1.3.5). A biotrophic interface for fungal-plant interaction is created by invagination of the plant cell plasma membrane. Only after successful plant penetration, the G2 cell cycle arrest is released and the fungus proliferates in planta by mitotic cell divisions and branching (Banuett and Herskowitz, 1996). Correct distribution of nuclei is achieved by formation of clamp cells (Scherer et al., 2006). Later during the infection, massive inter- and intracellular proliferation of the fungal hypha takes place in tumor tissue (Figure 1.3.6). After subsequent karyogamy, the hyphal sections fragment and differentiate into melanized diploid teliospores (Banuett and Herskowitz, 1996) (Figure 1.3.7). These spores are released into the environment and are able to germinate under suitable conditions. By meiosis and budding of from the promycelium, haploid sporidia are produced (Figure 1.3.1) (Christensen, 1963). 


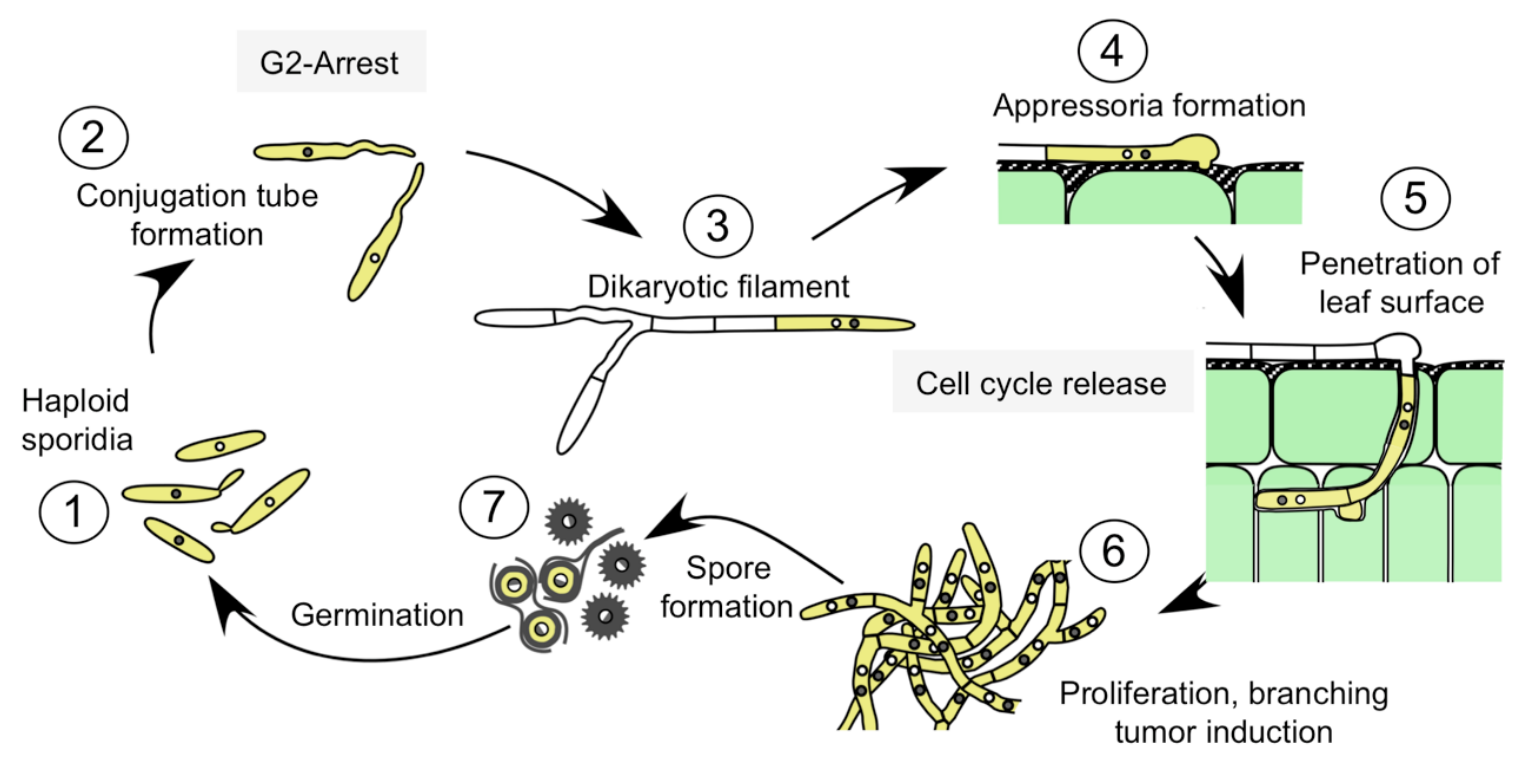

Figure 1.3 Life cycle of $\boldsymbol{U}$. maydis. Schematic diagram of the different developmental stages during sexual and pathogenic development: (1) Yeast-like growth of the haploid sporidia by budding. (2) Conjugation tube formation and cell fusion. (3) Filamentous growth of the dikaryotic filament. (4) Formation of appressoria on the plant surface. (5) Penetration of plant tissue and release of cell cycle arrest. (6) Proliferation and branching of the fungal hypha, followed by karyogamy. (7) Differentiation into melanized diploid teliospores. Life cycle modified from Kämper et al., 2006.

\subsubsection{Mating of $\boldsymbol{U}$. maydis}

Sexual reproduction of fungi is controlled by mating type loci. Basidiomycetes either have a bi- or a tetrapolar mating type system, whereby the tetrapolar system is predominant (Heitman, 2015). Fungi with a bipolar mating type system usually have bi-allelic mating type loci (a and $\alpha$ ). Tetrapolar mating type systems rely on two independent loci that specify the mating type. Both loci can be multiallelic resulting in thousands of different possible combinations/mating types (Heitman, 2015). The tetrapolar mating type system in $U$. maydis is characterized by two independent loci, called $a$ - and $b$-locus. These loci encode a pheromone/receptor system and homeodomain-transcription factors (Morrow and Fraser, 2009). The biallelic a-locus of $U$. maydis encodes the pheromone Mating factor a (Mfa1 or Mfa2) and the Pheromone receptor a (Pra1 or Pra2) (Figure 1.4). The pheromone precursor consists of 40 (Mfa1) or 38 (Mfa2) amino acids and is post-translationally modified by farnesylation and carboxyl methyl esterification of the C-terminus (Spellig et al., 1994). Additionally, the precursor is processed, resulting in a mature pheromone of 13 and 9 amino acids, respectively. Pra1 and Pra2 show similarities to STE3 pheromone receptors, contain seven transmembrane domains and are coupled to heterodimeric G-proteins (Bölker et al., 1992). 


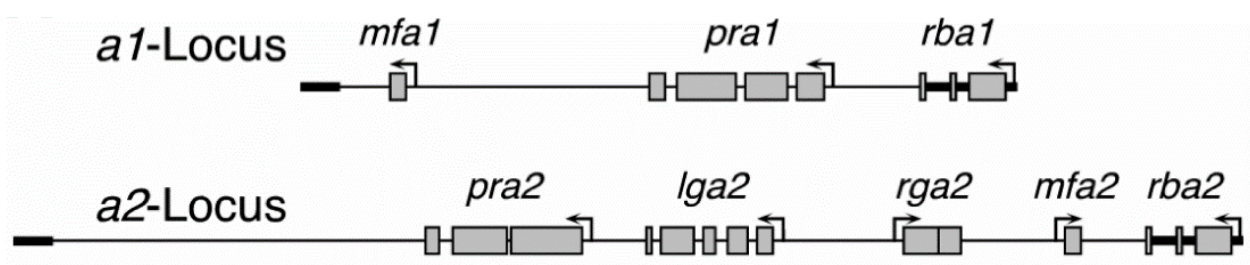

Figure 1.4 Schematic organization of the a1- and a2-locus. $m f a 1$ and $m f a 2$ encode the pheromone precursor. pral and pra2 encode for the two pheromone receptors. Modified from Brachmann, 2001.

A compatible pheromone is perceived by a receptor of the opposite mating type, resulting in a G2 cell cycle arrest and formation of conjugation tubes along the pheromone gradient (Spellig et al., 1994; Snetselaar et al., 1996; García-Muse et al., 2003). Transduction of the pheromone signal is achieved via two parallel cascades, the mitogen-activated protein-kinase (MAPK)- and the cAMP-dependent protein kinase A (PKA)-signaling pathway (see 1.3). Both converge on the key transcription factor Prf1 (see 1.4.1), which binds to pheromone response elements (PREs) in promoter regions of target genes. Thereby, expression of a large set of genes, including the $a$-mating type genes, is induced (Urban et al., 1996).

After fusion of two haploid cells, further pathogenic and sexual development is controlled by the multiallelic $b$-mating type locus (Urban et al., 1996). Each $b$-locus encodes a pair of homeodomain proteins called $b E$ and $b W$ (Figure 1.5A), that comprise of $473(\mathrm{bE}$ ) and 645 (bW) amino acids (Schulz et al., 1990; Gillissen et al., 1992). Both proteins consist of a highly variable N-terminal domain of about 120 amino acids and a conserved C-terminal region that contains the homeodomain motif (Gillissen et al., 1992; Kämper et al., 1995). The variable N-terminal part is responsible for non-self-recognition and formation of a heterodimer (Kronstad and Leong, 1990; Schulz et al., 1990; Gillissen et al., 1992). Only if the dikaryon contains nuclei with different $b$-mating type alleles (Figure 1.5B), bE and $\mathrm{bW}$ are able to dimerize and form the transcriptionally active bE/bW-heterodimer (Kämper et al., 1995).

Formation of an active b-heterodimer is necessary and sufficient to induce filamentous growth and pathogenic development (Bölker et al., 1995). An active heterodimer suppresses expression of the $a$-mating type genes and, as a master regulator, induces a transcriptional cascade. By that, filamentous growth and the G2 cell cycle arrest are maintained and the host plant surface can be penetrated by formation of appressoria (Kahmann and Kämper, 2004; Wahl et al., 2010b). Haploid strains containing compatible $b E$ and $b W$ genes are pathogenic and can infect the host and complete the lifecycle without the need of a mating partner (Bölker et al., 1995; Kämper et al., 2006). 
A

\section{B}
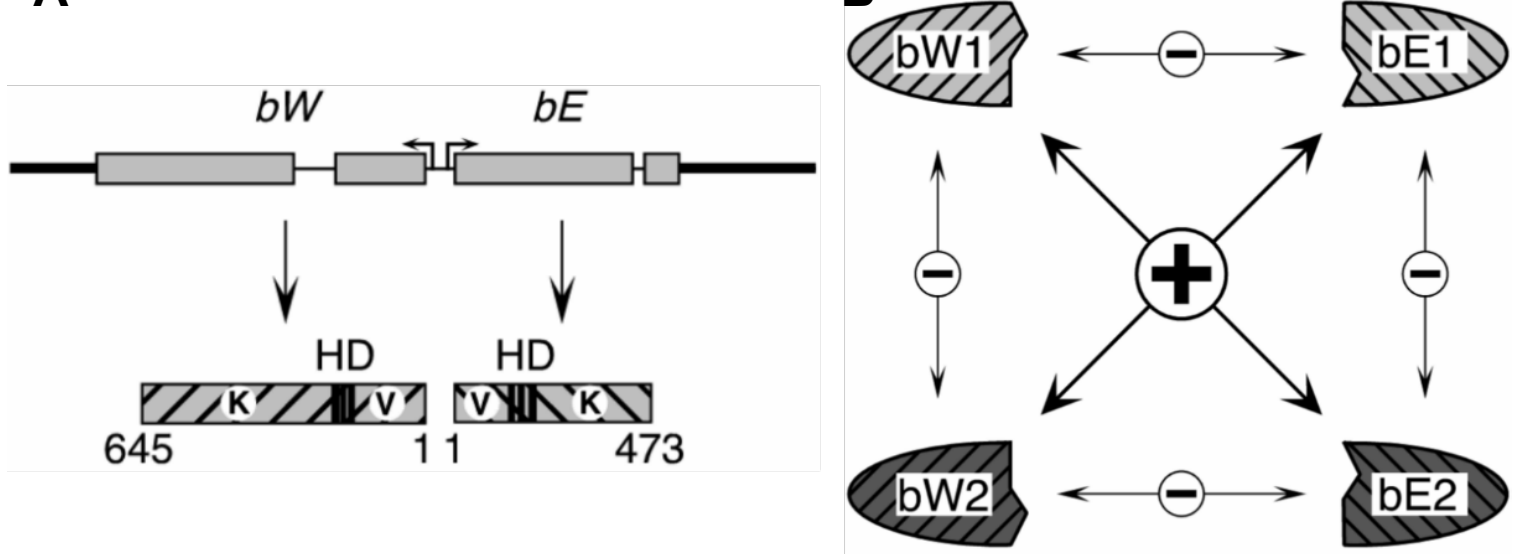

Figure 1.5 Schematic organization of the $\boldsymbol{b}$-locus. (A) The $b$-locus encodes the homeodomain proteins bW and $b E$. $\mathbf{V}$ indicates the variable N-terminal domains of $b E$ and $b W$. (B) Dimerization and formation of the $\mathrm{bE} / \mathrm{bW}$ heterodimer is only possible when the proteins derive from different mating type loci. Modified from Brachmann, 2001.

\subsection{MAPK Signaling}

MAPKs are evolutionary conserved components of eukaryotic signaling pathways. They control a multitude of different physiological processes, such as proliferation, differentiation, cell death and adaption to various stress conditions (Pearson et al., 2001; Cargnello and Roux, 2011). MAPK cascades are present in all eukaryotes and activated upon sensing of diverse signals including pheromones or cellular stress (Kültz and Burg, 1998; Widmann et al., 1999). A MAPK signaling cascade typically consists of three hierarchically acting kinases: the MAPK kinase kinase (MAPKKK) activates the MAPK kinase (MAPKK) that in turn activates the terminal MAPK. Common MAPK targets are transcription factors, which are phosphorylated to activate or inhibit expression of target genes. MAPKs are typically activated by phosphorylation of two specific threonine and tyrosine residues separated by a single amino acid. This conserved TXY motif is characteristic for MAPKs and essential for their enzymatic activity, as originally shown for the mammalian Extracellular Signal-regulated Protein Kinase 2 (ERK2) (Robbins et al., 1993). All members of the ERK1/2 subgroup of MAPKs are activated by extracellular signals and contain the TEY motif (Pearson et al., 2001).

MAPK pathways with varying in- and outputs use overlapping sets of components. Different strategies like organization into modules via scaffold proteins, docking site interactions, cross-pathway inhibition and isolation of components via stadium specific expression or localization are used to prevent undesirable leakage of signal or crosstalk between pathways (Bardwell, 2006; Dard and Peter, 2006; Saito, 2010; Good et al., 2011). 


\subsubsection{The pheromone response in $U$. maydis is mediated by MAPK- and PKA-dependent signaling cascades}

In $U$. maydis, signal transduction in response to pheromone perception is mediated by MAPK and PKA signaling cascades. Both pathways and their interaction control the processes of mating, morphogenesis and pathogenic development (Mayorga and Gold, 1999; Andrews et al., 2000; Mayorga and Gold, 2001; Müller et al., 2003). The MAPK cascade consists of the MAPKKK Kpp4 (Ubc4) (Andrews et al., 2000; Müller et al., 2003), MAPKK Fuz7 (Ubc5) (Banuett and Herskowitz, 1994) and MAPK Kpp2 (Ubc3) (Mayorga and Gold, 1999; Müller et al., 1999) (Figure 1.6). The kinases were initially identified as Ustilago bypass of cyclase proteins (Ubc3, Ubc4 and Ubc5), because they can suppress the filamentous phenotype of the adenylate cyclase (uacl) mutant of U. maydis (Gold et al., 1994; Mayorga and Gold, 1998; Mayorga and Gold, 1999). Two additional $u b c$ genes, $u b c 1$ and $u b c 2$, have been identified encoding the regulatory subunit of the protein kinase $\mathrm{A}$ (PKA) Adr1 and a Ste50-like protein (Gold et al., 1997). The cAMP-dependent PKA cascade and the pheromone dependent MAPK signaling cascade act in parallel and are interconnected (Mayorga and Gold, 1999). Ubc2, is a novel adaptor protein functioning upstream of the MAPK module (Mayorga and Gold, 1999; Mayorga and Gold, 2001; Klosterman et al., 2008). The N-terminal part of Ubc2 contains a sterile $\alpha$ motif (SAM) domain and a ras association (RA) domain (Mayorga and Gold, 2001). Both domains are potentially involved in protein-protein interactions and commonly present in Ste50-like fungal adaptor proteins. Ubc2 physically interacts with the MAPKKK Kpp4 via the SAM domain present in its N-terminus (Müller et al., 2003; Klosterman et al., 2008). The presence of an RA domain suggests a potential interaction with Ras or Ras-like proteins, including the family of small GTPases/GTP-binding proteins. U. maydis contains two Ras proteins, one of them, Ras2, functions upstream of the MAPK cascade (Lee and Kronstad, 2002), but interaction of Ras 2 and the adaptor Ubc2 could not be shown. Both, the SAM and the RA domain of $\mathrm{Ubc} 2$ are essential for filamentous growth (Klosterman et al., 2008). Pheromone binding to the cognate receptor activates the MAPK module resulting in phosphorylation and thereby activation of the terminal MAPK Kpp2 (Figure 1.6). Kpp2 is required for transcriptional and morphological response to a pheromone signal and thus for the mating process (Müller et al., 1999). It phosphorylates the pheromone response factor 1 (Prf1), which in turn induces expression of a defined set of pheromone-responsive genes 
(for details, see 1.4.1). By contrast, formation of conjugation tubes and appressoria as well as induction of the G2 cell cycle arrest are controlled by Kpp2 but are independent of Prf1 (García-Muse et al., 2003; Müller et al., 2003). While Kpp2 phosphorylation and its kinase activity are necessary to induce conjugation tube formation, the transcriptional response to the pheromone stimulus only depends on phosphorylation of Kpp2 and is independent of its kinase activity (Müller et al., 2003). Initiation of a G2 cell cycle arrest ensures synchronization of the cell cycle during conjugation tube formation and cell/cell fusion (García-Muse et al., 2003).

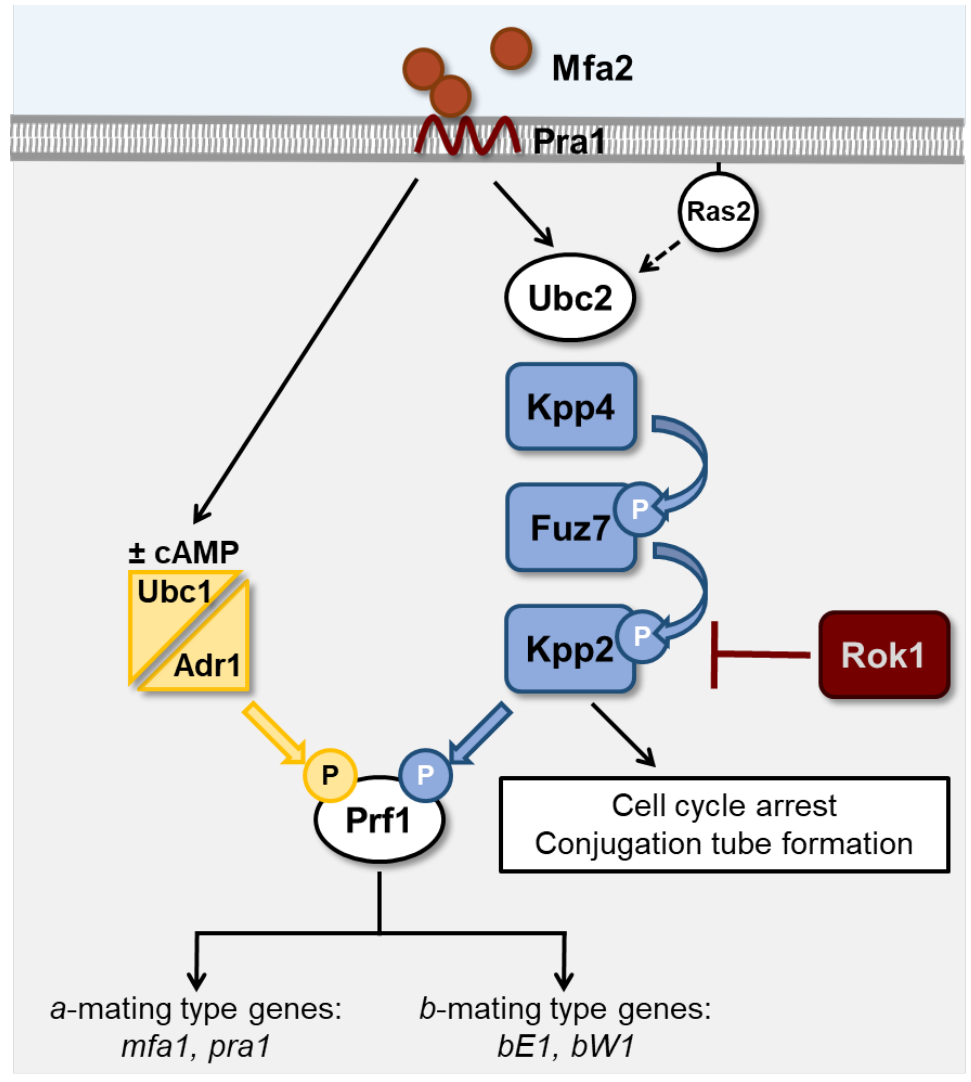

Figure 1.6 Schematic picture of the pheromone-dependent MAPK signaling cascade. Binding of the pheromone Mfa2 (Mfa1) to the compatible pheromone receptor Pral (Pra2) activates the adaptor protein Ubc2. Ubc2 physically interacts with the MAPKKK Kpp4 and thereby activates the MAPK module (Kpp4/Fuz7/Kpp2). The terminal MAPK Kpp2 phosphorylates Prf1 and, independent of Prf1, induces a G2 cell cycle arrest and formation of conjugation tubes. The cAMP-dependent PKA pathway is also activated upon pheromone sensing. The catalytic subunit of the PKA, Adr1, also phosphorylates and activates Prf1. Phosphorylated Prf1 induces expression of the $a$ - and $b$-mating type genes.

After fusion on the plant surface, the $\mathrm{bE} / \mathrm{bW}$ heterodimer is formed and a second MAPK, Kpp6, is Rbf1-dependently expressed (for details see 1.4.2 (Brachmann et al., 2003; Heimel et al., 2010b)). It has been shown that Kpp6 is part of the same MAPK module and also phosphorylated by the MAPKK Fuz7 (Brachmann et al., 2003; Di Stasio, 2009a). Although Kpp6 can partially substitute Kpp2 during cell fusion, it is not involved in pheromone signaling. Phosphorylation of Kpp6 is required for penetration of the plant surface after appressoria formation (Brachmann et al., 2003). It has been shown that the factors Synthetic high osmolarity sensitive (Sho1) and Multicopy suppressor of a budding defect 
(Msb2), which regulate appressorium development in response to a hydrophobic surface, function upstream of Kpp6 (Lanver et al., 2010a). Moreover, Sho1 directly interacts with

Kpp6, providing a rationale of how appressoria formation and plant penetration are connected and regulated (Mendoza-Mendoza et al., 2009a; Lanver et al., 2010a).

A third MAPK, Crk1, is also phosphorylated by the MAPKK Fuz7 (Garrido et al., 2004). Similar to Kpp2, Crk1 controls the transcriptional and morphological response to the pheromone signal, but is also required for filamentous growth after cell/cell fusion (Garrido et al., 2004). In addition to the common TEY phosphorylation motif, which is present in all three described MAPKs (Kpp2, Crk1, Kpp6), Crk1 contains two additional MAPK phosphorylation sites in its N-terminus. Phosphorylation of these sites is required for filament formation and most likely carried out by Kpp2 or Crk1 itself (Garrido et al., 2004). Constitutive active, unphosphorylatable or kinase-dead mutants of the MAPK module are available. The Fuz $7^{\mathrm{DD}}$ mutant protein harbors two point mutations resulting in the amino acid substitutions S259D and T263D to mimic constitutive phosphorylation of Fuz7 (Müller et al., 2003). Expression of $f u z^{D D}$ activates the MAPK module and thus mimics the pheromone response on transcriptional and morphological level (Müller et al., 2003). Other mutants, which are either kinase dead or non-phosphorylatable, are available for the MAPKs Kpp2, Kpp6 and Crk1. Kpp2AEF, Kpp6AEF and Crk1AEF contain two amino acid substitutions in the conserved TEY motif (Kpp2: T182A, Y184F; Kpp6: T355A, Y357F; Crk1: T253A, Y255F) rendering the MAPKs unphosphorylatable (Madhani et al., 1997; Müller et al., 1999; Brachmann et al., 2003; Garrido et al., 2004). The amino acid substitutions K50R (Kpp2), K220R (Kpp6) and K106R (Crk1) on the other hand lead to a kinase-dead protein which is defective in ATP binding (Brachmann, 2001; Müller, 2003; Garrido et al., 2004). By using these mutants, effects depending on a specific function, i.e. the kinase-activity or effect of MAPK phosphorylation can be analyzed.

\subsubsection{The dual specificity phosphatase Rok1 inhibits the pheromone response}

Phosphatases regulate kinase activity by dephosphorylation of specific amino acids. The group of MAP-Kinase-Phosphatases (MKP) specifically removes phosphate of tyrosine or/and threonine residues in the TXY motif of MAPKs. Three different types of MKPs exist: protein tyrosine phosphatases remove the phosphate of tyrosine residues, serine/threonine phosphatases dephosphorylate phosphothreonine and dual-specificity phosphatases (DSP) can remove tyrosine as well as threonine phosphorylation (Saxena and 
Mustelin, 2000). In U. maydis, the dual specificity phosphatase Rok1 regulates activity of the pheromone dependent MAPK signaling pathway. Rok1 dephosphorylates and thereby reduces activity of the MAPKs Kpp2 and Kpp6. Overexpression of rok1 interferes with the transition from budding to conjugation tube formation upon pheromone stimulus, most likely by preventing accumulation of phosphorylated Kpp2 (Di Stasio et al., 2009). Additionally, rokl overexpression reduces $b$-dependent filament formation and pathogenicity. By contrast, rokl deletion results in a strong filamentous phenotype independent of a pheromone stimulus or active $b$-signaling possibly due to increased activity of the MAPK module. $\Delta r o k l$ strains display increased b-gene expression, appressoria formation and more efficient colonization of the host leading to increased virulence of the fungus (Di Stasio et al., 2009). Rok1 harbors a protein-tyrosine phosphatase domain containing the highly conserved HCXXXXXXR motif characteristic for DSP (Denu et al., 1996; Di Stasio, 2009a). Additionally, the N-terminus of Rok1 contains one potential MAPK interaction site (D-box) and two potential MAPK phosphorylation sites. In the C-terminal extension, two of three total PEST sequences and three potential MAPK interaction sites are found (Jacobs et al., 1999; Di Stasio, 2009a). PEST sequences are rich in the amino acids proline, glutamate, serine and threonine and serve as proteolytic signals, leading to rapid protein degradation (Rogers et al., 1986; Rechsteiner, 1996). Furthermore, two potential Rok1 phosphorylation sites in the Nterminus of the protein have been predicted (Di Stasio, 2009a). MKPs like the DSP Rok1 are often regulated on a transcriptional as well as on a post-translational level (GonzálezRubio et al., 2019). rokl gene expression is induced upon pheromone stimulus/activation of the MAPK cascade, suggesting a role of Rok1 in a negative feedback loop controlling MAPK activity (Zarnack et al., 2008; Di Stasio et al., 2009). Post-translational regulation of MKPs is achieved by various mechanisms including protein-protein interactions, phosphorylation and reversible oxidation of the catalytic cysteine residue in the conserved DSP motif (Camps et al., 1998; Kamata et al., 2005; Patterson et al., 2009). Binding of DSP to their MAPK substrates can increase their catalytic activity by conformational changes (Camps et al., 1998; Fjeld et al., 2000). Moreover, phosphatases can be directly phosphorylated by their MAPK substrates leading to increased stability and activation (Brondello et al., 1999; Patterson et al., 2009). The exact mechanism of Rok1 regulation remains unknown, but possibly involves regulation on multiple levels as characteristic for proteins involved in fine-tuning of major signal cascades. 


\subsection{Prf1 and $b$-dependent signaling}

\subsubsection{The central transcription factor Prf1}

The high mobility group (HMG) transcription factor Prf1 is the central transcriptional regulator of pheromone signaling in U. maydis (Hartmann et al., 1996b; Kaffarnik et al., 2003). Prfl induces gene expression by binding to specific pheromone response elements (PREs) in promoter regions of target genes, including the $a$ - and $b$-mating type genes (Hartmann et al., 1996b; Urban et al., 1996). Prf1 is regulated on a transcriptional and on a post-translational level. Upon phosphorylation by the MAPK Kpp2 and/or the PKA Adr1 (Müller et al., 1999; Kaffarnik et al., 2003), it is activated and induces expression of different gene sets (Zarnack et al., 2008). Transcription of $p r f 1$ is regulated by interplay of at least four transcription factors downstream of the MAPKs Kpp2 and Crk1 and constitute a second level of regulation (García-Muse et al., 2003; Garrido et al., 2004). The transcription factors Rop1 and Hap2, Prf1 via autoregulation and an unknown factor controlled by Crk1 control prfl expression (Hartmann et al., 1996a; Hartmann et al., 1999; Garrido and Pérez-Martín, 2003; Garrido et al., 2004; Brefort et al., 2005; MendozaMendoza et al., 2009b). Prf1 induces its own transcription via binding to two pheromone response elements (PREs) in its promoter (Hartmann et al., 1996b; Urban et al., 1996). The unknown factor most likely integrates nutritional inputs and binds to an upstream activating sequence (UAS) in the promoter of prfl (Garrido and Pérez-Martín, 2003; Garrido et al., 2004).

\subsubsection{The b-dependent signaling cascade}

While the bE and bW proteins are already Prf1-dependently expressed in haploid sporidia during mating, the $\mathrm{bE} / \mathrm{bW}$ heterodimer can only form after fusion of two compatible cells (Kämper et al., 1995). Subsequent sexual and pathogenic development, maintenance of the G2 cell cycle arrest and filamentous growth depend on an active b-heterodimer (Bölker et al., 1995; Brachmann, 2001; Wahl et al., 2010b). Binding of bE/bW to conserved DNA sequences, so called b-binding sites $(b b s)$, in promoter regions of target genes induces expression of a large gene set (Romeis et al., 2000; Brachmann et al., 2001). 345 b-dependent genes have been identified by microarray analysis (Heimel et al., 2010b). Four of them, lgal (Romeis et al., 2000), frb52 (Brachmann et al., 2001), clp1 (Scherer et al., 2006) and rbfl (Heimel et al., 2010b) contain bbs in their promoters and are direct targets of the heterodimer. More than $90 \%$ of the b-dependent genes are regulated by the central Regulator of $b$-filament 1 (Rbf1), a $\mathrm{C} 2 \mathrm{H} 2$ zinc finger transcription factor (Heimel et al., 
2010b). These genes include additional regulators, such as the zinc finger transcription factor b-induced zinc finger 1 (Bizl), and the two homeodomain transcription factors Homeodomain protein 1 (Hdp1) and Homeodomain protein 2 (Hdp2) (Heimel et al., 2010b). Rbf1 is necessary and sufficient for maintenance of the b-mediated G2 cell cycle arrest, filamentous growth, formation of appressoria and penetration of the plant surface (Figure 1.7A) (Heimel et al., 2010b).
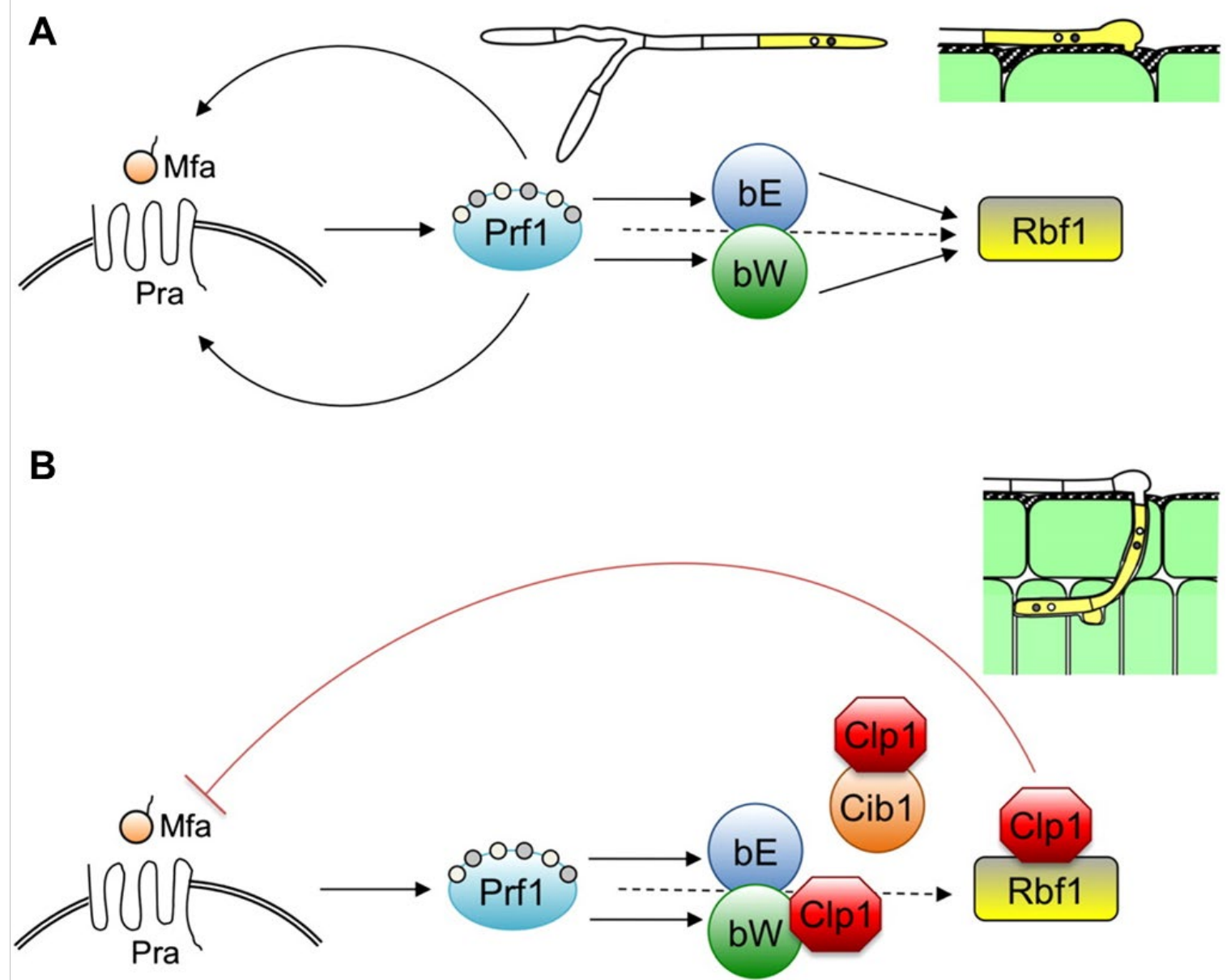

Figure 1.7 Prf1- and bE/bW-dependent regulation of initial stages of pathogenic development. (A) Formation of the active $\mathrm{bE} / \mathrm{bW}$ heterodimer induces expression of $r b f 1$. Rbf1 regulates maintenance of the G2 cell cycle arrest, elongation of the filament and appressoria formation to penetrate the plant surface. (B) After plant penetration, Clp1 interacts with bW and Rbfl and thereby represses a- and b-dependent gene regulation. Interaction with the bZIP transcription factor Cib1 is necessary for stabilization of Clp1 (see 1.5). Modified from Heimel et al., 2010.

The protein Clampless1 (Clp1) is also a direct $\mathrm{bE} / \mathrm{bW}$ target and required for release of the cell cycle arrest and proliferation in planta (Heimel et al., 2010a). clp1 mRNA can be detected rapidly after induction of the heterodimer, but the protein is only present after plant penetration (Scherer et al., 2006; Heimel et al., 2010a). Clp1 regulates development in planta by physical interaction with bW and Rbf1 (Heimel et al., 2010a). Interaction with bW blocks b-dependent gene expression and development (Scherer et al., 2006) while 
interaction with Rbf1 inhibits $a$-mating type gene expression and the morphological pheromone response (Figure 1.7B) (Heimel et al., 2010a). This releases the cell cycle block after plant penetration and facilitates proliferation of the fungus (Heimel et al., 2013).

\subsection{The UPR in U. maydis}

During penetration of the plant, the host plasma membrane invaginates and covers the fungal hyphae creating an interaction zone. This allows efficient secretion of effectors to suppress the plant defense and redirect nutrients to the fungal hyphae (Kämper et al., 2006; Skibbe et al., 2010; Djamei and Kahmann, 2012). Overall, 554 secreted proteins have been predicted for U. maydis (Müller et al., 2008). All of them have to be synthesized and further modified resulting in high stress levels imposed on the ER and secretory machinery. This is counteracted by activation of the UPR ((Walter and Ron, 2011), for details see 1.1). The Clp1 interacting bZIP1 (Cib1) is the main regulator of the UPR in U. maydis (Heimel et al., 2010a). Cib1 is the homolog of Hac1p in yeast and XBP1 in higher eukaryotes (Heimel et al., 2013). Similar to yeast and mammals, un- and misfolded proteins in the ER lumen are sensed by the functional homolog of the RNase/kinase Irelp. Activated Ire1 processes cibl mRNA via unconventional splicing (Heimel et al., 2013). The spliced transcript is translated into the active transcription factor, $\mathrm{Cib}^{\mathrm{s}}$, that induces expression of UPR target genes.

Beside its function as central regulator of the UPR, Cib1 also coordinates biotrophic development (Heimel et al., 2013). After penetration of the plant surface, Cib1 physically interacts with Clp1 and thereby stabilizes the protein (Heimel et al., 2010a; Heimel et al., 2013). This leads to accumulation of Clp1 and to the Clp1-mediated release of the cell cycle block and proliferation in planta (Heimel et al., 2013). Deletion of cibl results in a loss of virulence and resembles the $c l p 1$ deletion phenotype where development stops directly after penetration of the plant surface (Scherer et al., 2006; Heimel et al., 2010a).

Correct timing of UPR activity seems to be crucial for pathogenic development of U. maydis. Premature UPR activation inhibits expression of $b E, b W$ and $r b f l$ and suppresses $b$-dependent filament formation (Heimel et al., 2013). The transition from yeast-like growth to formation of the infectious dikaryotic filament is blocked. Neither constitutive $b E / b W$ expression nor $c l p 1$ deletion completely restored filament formation in $c i b 1^{\text {s }}$ expressing strains (Heimel et al., 2013). Thus, the UPR inhibits the b-pathway by at 
Chapter 1 | Introduction

least one additional, yet unknown, mechanism and is part of a regulatory network that coordinates pathogenic development of $U$. maydis (Heimel et al., 2013).

\subsection{Aim of this study}

The UPR is a highly conserved eukaryotic signaling pathway crucial for maintenance of ER homeostasis during growth in planta. Activation of the UPR after plant penetration is necessary for efficient effector secretion and supports development and proliferation. By contrast, premature activation of the UPR blocks early steps of pathogenic development by suppressing expression of the main regulators of filamentous growth. Thus, correct timing of UPR activation is crucial for developmental progression and pathogenicity of the fungus. It is known that the UPR and the developmental pathways interact at multiple levels. However, it has not been addressed where the crosstalk takes place and how this crosstalk affects pathogenic development in planta. The aim of this study is to identify the signal hub(s) that mediate crosstalk between the UPR and the developmental pathways and to analyze in which way the signaling cascade operating upstream of the $\mathrm{bE} / \mathrm{bW}$-heterodimer is affected. The role of the identified signal hub(s) and its function during pathogenic development in planta will be analyzed. A conditional gene expression system will be established to specifically address protein functions and the relevance of crosstalk between signaling pathway components during pathogenic growth in planta. 


\section{Chapter 2 | Transcripts and Tumors}

Results described in chapter 2 were published in F1000 Research (doi: 10.12688/f1000research.16404.1):

\section{Transcripts and Tumors: Regulatory and metabolic programming during biotrophic phytopathogenesis}

Lara Schmitz $^{1 *}$, Sean McCotter ${ }^{2 *}$, Matthias Kretschmer $^{2 *}$, James Kronstad $^{2}$, Kai Heimel $^{1}$

${ }^{1}$ Georg-August-University-Göttingen, Institute for Microbiology and Genetics

Department of Molecular Microbiology and Genetics, Grisebachstr. 8

D-37077 Göttingen, Germany

${ }^{2}$ Michael Smith Laboratories, Department of Microbiology and Immunology, University of British Columbia, Vancouver, BC, Canada V6T 1Z4

*These authors contributed equally to the review.

\section{Author contribution:}

JK wrote chapter 2.1. LS wrote chapter 2.3. SM wrote chapter 2.5. MK wrote chapter 2.4. $\mathrm{KH}$ wrote chapter 2.2 and 2.6. LS and $\mathrm{KH}$ designed the figures. 


\subsection{Abstract}

Biotrophic fungal pathogens of plants must sense and adapt to the host environment to complete their life cycles. Recent transcriptome studies of the infection of maize by the biotrophic pathogen Ustilago maydis are providing molecular insights into an ordered program of changes in gene expression and the deployment of effectors, as well as key features of nutrient acquisition. In particular, the transcriptome data provide a deeper appreciation of the complexity of the transcription factor network that controls the biotrophic program of invasion, proliferation and sporulation. Additionally, transcriptome analysis during tumor formation, a key late stage in the life cycle, revealed features of the remodeling of host and pathogen metabolism that may support the formation of tremendous numbers of spores. Transcriptome studies are also appearing for other smut species during interactions with their hosts thereby providing opportunities for comparative approaches to understand biotrophic adaptation.

Keywords: Ustilago maydis, transcriptome, RNAseq, pathogenicity, regulators, effectors, nutrients, metabolism

\subsection{Introduction}

Fungal phytopathogens in the order Ustilaginales generally attack cereal and grass plants to cause smut diseases, so named because of the tremendous masses of sooty spores produced in infected tissue. Biotrophic pathogens such as the smut fungi are obligately dependent on living hosts to successfully complete their life cycles. Effective adaptation to the host environment is therefore critical for overcoming the plant immune response and successfully exploiting host nutrients through remodeling of metabolism and effective competition. The mechanisms by which biotrophic pathogens manipulate host metabolism to divert carbon, nitrogen or micronutrients such as iron for their own use are starting to be identified. Among the Ustilaginales, the Zea mays (maize) pathogen Ustilago maydis has emerged as an experimentally tractable model for studying the adaptation of biotrophic fungal pathogens to the host environment. As an example of the intricate interaction with the plant host, the life cycle of $U$. maydis involves germination of diploid teliospores on host tissue with subsequent mating between haploid meiotic progeny to form an invasive filamentous cell type (Brefort et al., 2009b; Heimel et al., 2010b). The filaments then form 
appressoria (invasion structures) to penetrate host tissue with subsequent extensive proliferation, induction of tumors (galls) and eventual formation of melanized teliospores (Brefort et al., 2009; Lanver et al., 2014) (Figure 2.1). A large number of effectors are predicted for $U$. maydis and these proteins are thought to play key roles in managing the infection process. To date, characterized functions include the effectors Cmu1, Pit2, Pep1 and Tin 2 that influence host defense, and See1 and Rsp3 that influence tumor progression and defense, respectively (Lanver et al., 2017; Ma et al., 2018).

In this review we focus on recent genome-wide transcriptome studies of the infection

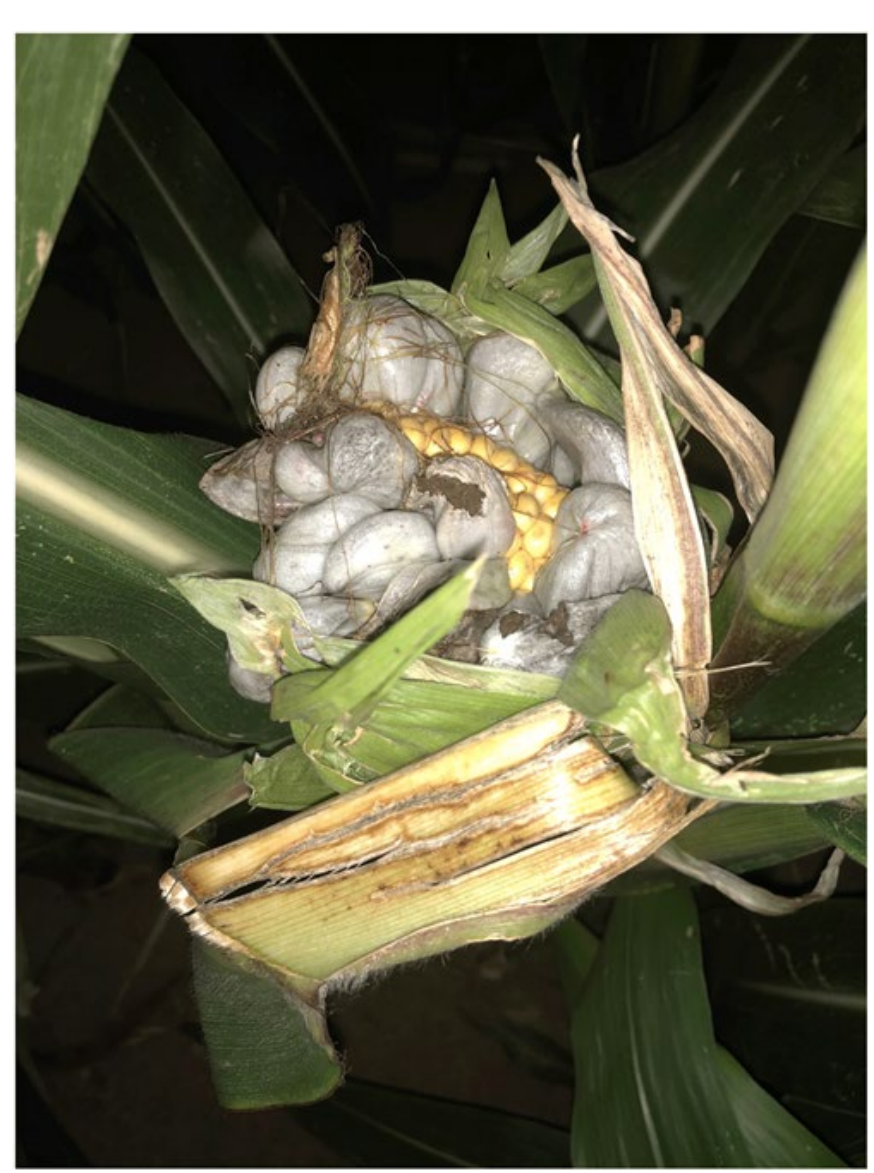

Figure 2.1 Tumor formation on maize by $U$. maydis. An infected maize cob found in a cornfield close to Göttingen, Germany, in September 2018. At the end of the growing season, infected kernels give rise to greatly enlarged and bulbous plant tumors filled with black teliospores. Tumors can develop on all aerial parts of the plant but are most prominent in infected cobs. process that have provided insights into the transcriptional regulation associated with disease, the deployment of effectors by $U$. maydis to manage the infection process, and the remodeling of host metabolism during fungal proliferation. Although not covered here, earlier studies also examined the transcriptome of $U$. maydis in culture and during infection (Eichhorn et al., 2006; Doehlemann et al., 2008; Heimel et al., 2010b; Horst et al., 2010; Skibbe et al., 2010; Wahl et al., 2010b; Zahiri et al., 2010; Jonkers et al., 2012; MartínezSoto and Ruiz-Herrera, 2013; FrancoFrías et al., 2014; Lanver et al., 2014; Islamovic et al., 2015; Tollot et al., 2016; León-Ramírez et al., 2017; Sánchez-Arreguin et al., 2017).

We also refer readers to a wealth of primary literature and recent reviews on the role of transcription factors, regulators and effectors in the disease process for U. maydis (Flor-Parra et al., 2006; Heimel et al., 2010a; Lanver et al., 2010a; Wahl et al., 2010b; Zahiri et al., 2010; Heimel et al., 2013; Tollot et al., 2016). 


\subsection{Transcriptome analysis throughout the infection process.}

A recent time-resolved and genome-wide study of transcriptional changes occurring during the $U$. maydis/maize interaction provides detailed insights into the processes underlying infection, tumor formation and sporogenesis (Lanver et al., 2018). Among 14 regulatory modules identified, three gene sets/developmental programs were "virulence-specific" and upregulated during discrete developmental stages (Figure 2.2). These gene sets are specifically expressed during: 1) growth on the plant surface (early); 2) biotrophic development in planta (middle) and; 3) tumor formation (late/sporogenesis). In all three sets, genes encoding secreted proteins (effectors) are significantly overrepresented, emphasizing their critical role during biotrophic development. Metabolic changes are also predicted and, importantly, several transcription factors with a strong connection to each respective module were identified as potential key regulators (Figure 2.2).

\begin{tabular}{|c|c|c|c|}
\hline & $\begin{array}{l}\text { Plant surface } \\
\text { (0.5-1 dpi) }\end{array}$ & $\begin{array}{l}\text { Proliferation in planta } \\
\text { (2-4 dpi) }\end{array}$ & $\begin{array}{l}\text { Tumor and spore formation } \\
\qquad \text { (4-8 dpi) }\end{array}$ \\
\hline $\begin{array}{l}\text { Potential key } \\
\text { regulators }\end{array}$ & $\begin{array}{l}\mathrm{bE} / \mathrm{bW} \\
\mathrm{Rbf1}\end{array}$ & $\begin{array}{l}\text { Hdp2 } \\
\text { Biz1 }\end{array}$ & $\begin{array}{l}\text { Nlt1 } \\
\text { Fox1 } \\
\text { Ros1 }\end{array}$ \\
\hline Effectors & $\begin{array}{l}49 \text { potential effectors } \\
\text { Pep1 }\end{array}$ & $\begin{array}{c}228 \text { potential effectors } \\
\text { Pep1 } \\
\text { Pit2 } \\
\text { Cmu1 } \\
\text { Tin2 } \\
\text { Rsp3 } \\
\text { See1 }\end{array}$ & $\begin{array}{l}38 \text { potential effectors } \\
\text { See } 1\end{array}$ \\
\hline Nutrient assimilation & $\begin{array}{l}\text { - Hydrolysis and uptake of plant cell } \\
\text { wall components }\end{array}$ & $\begin{array}{l}\text {-Ammonium transp.: Ump1, Ump2 } \\
\text { - Oligopeptide transp.: Opt2, Opt3, } \\
\text { Opt4 } \\
\text { - Sucrose transporter Srt1 } \\
\text { - Glucose transporter Hxt1 } \\
\text { - Aspartic proteases }\end{array}$ & $\begin{array}{l}\text { - Protein catabolism } \\
\text { - Autophagy } \\
\text { - lipid transport and metabolism } \\
\text { - nitrogen and carbon catabolite } \\
\text { derepressors }\end{array}$ \\
\hline
\end{tabular}

Figure 2.2 Regulators, effectors, and metabolism during Ustilago maydis infection of maize. Overview of transcriptional regulation, the timing of effector function, and metabolic changes and requirements during early, middle, and late stages of the U. maydis-maize interaction. DPI, days post inoculation.

For the early stage of infection, it is known that the zinc finger transcription factor Rbf1 is required and sufficient for the initial steps of pathogenic development (Heimel et al., 2010b; Heimel et al., 2010a). The recent work by Lanver et al., 2018, revealed that Rbf1 shows a strong regulatory connectivity to genes expressed at the early stage (0.5-1 days post inoculation). Among the 398 genes that are upregulated, 49 encode for secreted proteins that are enriched for hydrolytic enzymes with a predicted function in appressoriamediated plant penetration. Several genes induced on the leaf surface encode sugar and 
nitrogen-related transporters, indicating priming of $U$. maydis for altered nutrient availability during plant colonization.

The subsequent middle phase of biotrophic development (2-4 DPI) involves the reinitiation of fungal proliferation, establishment of a compatible biotrophic interaction and the beginning of tumor formation. Enriched functional categories in the transcriptome include nitrogen and carbon source-related processes, and components of the secretory pathway including the unfolded protein response (UPR) (Lanver et al., 2018) Transcriptional regulation during the biotrophic stage is modulated by direct interaction between UPR components and key regulators of fungal development and pathogenicity (Heimel et al., 2013). Potential transcriptional regulators of this stage include the Rbf1regulated homeodomain transcription factor Hdp2 (Heimel et al., 2010b; Lanver et al., 2014) and the $\mathrm{C} 2 \mathrm{H} 2$-zinc finger transcription factor Biz1 (Flor-Parra et al., 2006).

Among the 228 genes for effectors that were upregulated in the middle stage, 153 lack functional signatures. However, the characterized genes encoding the effectors pep 1, pit2, cmu1, seel and tin2, are highly induced at this stage, and these are required for full virulence of $U$. maydis and/or suppression of plant defense reactions (reviewed in Lanver et al., 2017). Cell type-specific transcriptome analysis revealed that genes encoding the core effectors Pep1, Pit2, Cmu1 and Tin2 are expressed independent of the plant organ or cell type, whereas See1 is exclusively expressed in leaf tissue (Schilling et al., 2014; Redkar et al., 2015). Tumor formation occurs by distinct mechanisms in hyperplastic bundle sheath derived tumor cells (HPT) or hypertrophic mesophyll derived tumor cells (HTT). See1 mediates only bundle-sheath cell-derived tumor formation, whereas mesophyll-derived tumor formation is independent of See1 (Matei et al., 2018).

During tumor formation in the middle stage, the ammonium transporters Ump1 and Ump2, and the oligopeptide transporters Opt2, Opt3, and Opt4, appear to mediate nitrogen acquisition. Ump1, Ump2, and Opt2 are induced already on the leaf surface and further upregulated in planta (Lanver et al., 2018; Paul et al., 2018). The fact that two secreted aspartic proteases are co-expressed with the OPTs suggests that breakdown of extracellular proteins and peptide uptake may be part of the biotrophic developmental program of $U$. maydis (Lanver et al., 2018). Carbon assimilation during biotrophic development is known to mainly occur via the high-affinity sucrose and glucose transporters Srt1 and Hxt1, respectively. While Srt1 is strongly induced during plant colonization, Hxt1 expression is 
independent of the fungus/plant interaction (Wahl et al., 2010a; Schuler et al., 2015).

At later developmental stages (4-8 DPI), a third wave of effectors guides tumor maturation and production of melanized teliospores. One important regulator after establishment of the biotrophic interaction is the APSES transcription factor Nlt1. Nlt1 appears to guide tumor formation on leaf tissue, but not anthocyanin and tumor formation on the base of the stem (Lanver et al., 2018). Another factor contributing to the regulation of effector gene expression at the later stage is the forkhead transcription factor Fox1. Fox1 is required for attenuation of plant defense responses and for full expression of 141 genes, of which 38 encode potential effectors, as well as several proteins involved in sugar processing and transport, and secondary metabolism (Zahiri et al., 2010). Genes encoding effectors that are specifically expressed at late stages of biotrophic development are largely unexplored functionally. Potentially, they function to sustain tumor development and guide spore formation, as well as formation of a mucilaginous matrix in which spores are embedded. A key regulator of these late-stage events is the DNA-binding WOPR protein Ros1. Ros1 function is essential for sporogenesis and the majority of Ros1 target genes are involved in metabolism and cellular transport. The Ros1-dependent induction of late effector genes and repression of early effector-encoding genes indicates that Ros1-mediated regulation occurs by direct and indirect mechanisms, involving additional regulators (Morrison et al., 2012). As described below, further insights into late stage changes in both host and pathogen gene regulation were obtained by examining transcriptomes specifically in tumors.

\subsection{Transcriptome changes reveal extensive metabolic remodeling in tumors.}

Recent transcriptome profiling experiments of the late stage of infection when tumors were evident identified host genes that were up- (4086) or down- (5237) regulated (Kretschmer et al., 2017a; Kretschmer et al., 2017b). Downregulated functional categories included metabolic pathways for amino acids, organic acids, lipids, and photosynthesis. By contrast, upregulated functional categories were related to plant defense, lipid and carbohydrate metabolism. As expected for proliferating maize cells during tumor formation, meristem maintenance functions were upregulated during infection. Additionally, maize transcription factors that are normally expressed in leaf tissue during plant development were downregulated in infected plants. In contrast, transcription factors normally expressed in flowers were upregulated in tumor tissue. Thus, $U$. maydis appears to inhibit the transition 
to adult tissue development and instead induces a flowering program. Chloroplast functions related to vegetative tissue, such as photosynthesis, lipid production, amino acid formation and secondary metabolism, were downregulated while reproductive functions such as sucrose and starch metabolism were upregulated during infection. Maize mutants in host components related to the vegetative to flowering transition, the biogenesis of the chloroplast, or starch formation, respectively, all showed altered susceptibility to $U$. maydis. For example, impaired chloroplast formation in the whyl mutant led to increased susceptibility, while the inactive/delayed transition to a flowering state (id1) and the reduced starch formation leading to lower sink capacity (su1) led to increased plant resistance (Kretschmer et al., 2017a; Kretschmer et al., 2017b).

Consistent with earlier work, transcriptome profiling of tumor tissue supported an important role of sugar transporters for metabolic exploitation of the host (Wahl et al., 2010a; Schuler et al., 2015; Kretschmer et al., 2017a). Hexoses and other sugars represent major carbon sources for $U$. maydis, and the fungus possesses 19 proteins with similarities to sugar/hexose-like transporters. As mentioned above, transporters such as Hxt1 and Srt1 play important roles during infection. Hxt1 is constitutively expressed and has transport activity mainly for glucose but also fructose and mannose. As a high-affinity glucose transporter, Hxt1 also possesses glucose-sensing activity and competes with the host for glucose molecules freed from sucrose by invertase. Previous work involving the deletion of the gene for this transporter or expression of a constitutive active (sensing but not transport activity) version resulted in strains with reduced or fully abolished virulence, respectively (Schuler et al., 2015). As indicated, the transporter Srt1 is only induced in planta and transports sucrose. Based on its extremely high-affinity to sucrose (up to 200fold vs. a typical plant transporter) it outcompetes the sucrose transport functions of the host. The virulence of deletion strains for srt 1 was strongly reduced (Wahl et al., 2010a). Deletion of both $h x t 1$ and srt 1 further reduced fungal virulence thus indicating a simultaneous uptake and use of sucrose and glucose by the pathogen during infection. Other carbon sources such as organic acids (fatty acids or carboxylic acids) may also contribute to the carbon supply of the pathogen at early time points of infection on the cuticle and at a late stage during spore formation (Kretschmer et al., 2012). Monocarboxylic acids are a carbon source for $U$. maydis in vitro but can have detrimental effects on fungal longevity and ROS resistance (Kretschmer et al., 2018). Ustilago is known to inhibit the transition to $\mathrm{C} 4$ metabolism that is dependent on the dicarboxylate malate as transport acid between 
cells. However, it is still unknown how and if different host derived carboxylic acids affect fungal virulence.

In general, the transcriptome analyses of infected tissue support the idea that control of chloroplast functions is a critical aspect of the infection of maize by U. maydis. Moreover, primary and secondary metabolic functions, and plant defense contributions of the chloroplast appear to be suppressed or altered during infection. In particular, chloroplasts were observed to accumulate high amounts of starch, consistent with an overall increased starch content in tumor tissue (Doehlemann et al., 2008; Kretschmer et al., 2017a; Matei et al., 2018). However, the mechanisms by which U. maydis impacts the chloroplast are still unknown.

\subsection{Beyond $U$. maydis: transcriptome studies with other smut fungi}

A number of transcriptome studies have also been carried out for host-pathogen interactions involving other species in the Ustilaginales. For example, Que et al., 2014, examined the interaction between the sugarcane smut Sporisorium scitamineum and its host Saccharum officinarum, comparing the transcriptional responses of resistant (Yacheng05-179) and susceptible ("ROC"22) plant lines. This study focused on the transcriptional responses of the host at 24, 48 and 120 hours after inoculation (HAI) in resistant and susceptible cultivars. The susceptible line showed an overall increased number of differentially expressed genes (DEGs), whereas the resistant cultivar triggers a rapid response to pathogen infection. Consistently, most DEGs were associated with stress and defense responses to the pathogen. The resistant cultivar revealed a rapid peak in DEGs at 48 hours, and more DEGs were associated with disease resistance when compared to the susceptible cultivar. The susceptible cultivar showed a similar accumulation of disease resistanceassociated DEGs at the 120 hour time point, highlighting the difference in the timing of the response to infection by the resistant and susceptible lines. Categories of genes showing differential expression encoded metabolic functions associated with resistance, hormone signaling, flavonoid biosynthesis, cell wall fortification, and defense-related transcription. Interestingly, a total of 26 host chitinases were differentially expressed in the $S$. scitamineum-infected plants. Expression of two of the chitinases in tobacco led to increased disease resistance against Fusarium solani and Botrytis cinerea. 
In a more recent transcriptome study, Wang et al., 2017, investigated the interaction between the edible culm-gall forming fungus Ustilago esculenta and the host Zizania latifolia, an aquatic grass. Interestingly, infected plant material is harvested as a prized vegetable in Asia. The transcriptome study compared infections for two strains of the fungus, the teliospore-forming strain Huijiao and the non-sporulating strain Jiaobai. In general, DEGs were more frequently observed in plants infected with the Jiaobai strain versus the Huijiao strain, although the proportion of fungal transcripts was lower in the former infection. Huijiao-infected plants exhibited a peak of 57 percent of total transcripts of fungal origin at 10 days after swelling (DAS), compared to only $\sim 5$ percent for Jiaobai.

With regard to host gene expression, clear differences were observed over the course of infection. That is, for plants infected with the Jiaobai and Huijiao strains, 18 and 12 percent of the host genes were upregulated at each time point, while 52 and 60 percent were upregulated only at specific time points in comparison to uninfected controls. Only a small fraction of the Z. latifolia genes were found to be consistently upregulated across all time points: i.e., $<1$ percent for infection with Huijiao and $\sim 2$ percent for infection with Jiaobai indicating that most Z. latifolia genes are expressed in a developmental-stage specific manner. A similar expression pattern was also observed for downregulated host genes. Additionally, 170 and 205 DEGs with potential roles in culm-gall formation were identified at 1 and 10 DAS, respectively. These genes encoded proteins with predicted roles in primary and secondary metabolism, as well as in plant-pathogen interactions and plant hormone signal transduction.

Comparisons between host genes consistently upregulated by Jiaobai and Huijiao point to an important role for developmental stage-specific gene expression in culm gall formation, this time on the part of the pathogen. This observation of stage specific gene expression in the fungal pathogen mirrors the observations by Lanver et al., 2018, with regard to $U$. maydis. Applying the criteria described above for host genes, 78 and 109 pathogen genes were identified at 1 and 10 DAS that may play roles in culm gall formation. Fungal genes which were specifically upregulated at 1 DAS included un-annotated genes and genes coding for proteins with predicted functions in nucleotide binding, transcriptional regulation, hydrolase activity, transferase activity and transport. Genes specifically upregulated at 10 DAS fell into the same categories. 
In addition to $U$. maydis, other members of the Ustilaginales have been investigated as models for studying biotrophic phytopathology. For example, Rabe et al. (Kretschmer et al., 2012) carried out a comprehensive study of the genome, transcriptome and utility as a model system of the grass pathogen Ustilago bromivora and one of its hosts Brachypodium sp. Initially, a susceptible line, $\mathrm{Bd} 28$, was identified after screening a number of Brachypodium sp. accessions for susceptibility to U. bromivora. In addition to extensive characterization of $U$. bromivora and its interaction with the host, a transcriptome analysis compared fungal gene expression of $U$. bromivora in axenic culture with infected stems of $\mathrm{Bd} 28$. It was found that 7.3 percent of the identified fungal transcripts were upregulated while 16.8 percent were downregulated. It is notable that these percentages are far lower than those reported by Lanver et al., 2018, for differential expression of $U$. maydis genes during plant infection. For U. bromivora, transcripts encode potential effectors were enriched among upregulated transcripts during infection (30.8\%). In comparison, only $5.7 \%$ of the total transcripts overall were predicted to encode secreted proteins. Among the genes for predicted secreted proteins, $84.1 \%$ encoded proteins of unknown function, compared with a frequency of $46.4 \%$ for all of the proteins encoded by $U$. bromivora. This

finding is consistent with other studies indicating that most secreted effectors of plant pathogenic fungi are uncharacterized and poorly conserved between species (Lanver et al., 2018). The observation that all of the functionally characterized effectors identified in $U$. maydis also have homologs in U. bromivora highlights the importance of fungal model systems to investigate molecular principles of effector functions.

\subsection{Conclusions and future perspectives}

Overall, recent transcriptome profiling has revealed dynamic changes in transcription of pathogen genes encoding metabolic functions (e.g., for nitrogen and carbon metabolism) that likely reflect shifting environmental conditions throughout the disease process as $U$. maydis colonizes tissue, induces tumors, and sporulates. It is fascinating that the changes in pathogen gene expression are integrated with and driven by effector-mediated manipulation of host cell metabolism and plant immunity. Time-resolved analyses greatly increase our understanding of individual stages of pathogenic development and how transitions between those are coordinated. Future work will exploit the accumulated wealth of data to experimentally address predictions about pathogen effectors and their regulators, and the importance of specific metabolic changes for both the pathogen and the host. For 
example, it is of keen interest to understand how different host environments are sensed and translated into adapted transcriptional programs. Also, future work will further examine the details of tumor formation. In this regard, comparisons between non-tumor forming smuts, such as U. esculenta, Sporisorium reilianum, and Ustilago hordei, and U. maydis may provide clues about the timing of tumor induction, and the corresponding specific pathogen and host functions. The sophisticated molecular tools established for U. maydis, and the emerging tools for $U$. bromivora, will provide novel insights that are relevant specifically for tumor induction and sporulation by other smut fungi, as well as generally for the mechanisms of biotrophy.
Abbreviations
(DAS) days after swelling
(DEGs) differentially expressed genes
(DPI) days post inoculation
(HAI) hours after inoculation
(HTT) hypertrophic tumor cell
(HPT) hyperplastic tumor cell
(UPR) unfolded protein response

\section{Disclosure}

The authors declare that they have no disclosures.

\section{Grand information}

Our research on Ustilago maydis is supported by a Discovery grant (to J.W.K.) and a CREATE training award (to the PRoTECT group) from the Natural Sciences and Engineering Research Council of Canada, and by grants from the Deutsche Forschungsgemeinschaft (DFG) to (K.H) and the DFG-funded International Research Training Group (PRoTECT). 
Chapter $2 \mid$ Transcripts and Tumors

\section{Acknowledgements}

We apologize to those colleagues whose work could not be cited due to space limitations. The picture showing a maize cob infected with $U$. maydis was generously provided by Melina A. Schwier.

\subsection{References}

Brefort, T., Doehlemann, G., Mendoza-Mendoza, A., Reissmann, S., Djamei, A., and Kahmann, R. (2009) Ustilago maydis as a Pathogen . Annu Rev Phytopathol 47: 423-445.

Doehlemann, G., Wahl, R., Horst, R.J., Voll, L.M., Poree, F., Stitt, M., et al. (2008) Reprogramming a maize plant: transcriptional and metabolic changes induced by the fungal biotroph Ustilago maydis. The Plant Journal 181-195.

Eichhorn, H., Lessing, F., Winterberg, B., Schirawski, J., Kämper, J., and Kahmann, R. (2006) A Ferroxidation/Permeation Iron Uptake System Is Required for Virulence in Ustilago maydis. Plant Cell 18: 3332-3345.

Flor-Parra, I., Vranes, M., Kämper, J., and Perez-Martin, J. (2006) Biz1, a Zinc Finger Protein Required for Plant Invasion by Ustilago maydis, Regulates the Levels of a Mitotic Cyclin. Plant Cell Online 18: 2369-2387.

Franco-Frías, E., Ruiz-Herrera, J., and Aréchiga-Carvajal, E.T. (2014) Transcriptomic analysis of the role of Rim101/PacC in the adaptation of Ustilago maydis to an alkaline environment. Microbiology 160: 1985-1998.

Heimel, K., Freitag, J., Hampel, M., Ast, J., Bolker, M., and Kamper, J. (2013) Crosstalk between the Unfolded Protein Response and Pathways That Regulate Pathogenic Development in Ustilago maydis. Plant Cell 25: 4262-4277

Heimel, K., Scherer, M., Schuler, D., Kamper, J., and Kämper, J. (2010a) The Ustilago maydis Clp1 Protein Orchestrates Pheromone and $b$-Dependent Signaling Pathways to Coordinate the Cell Cycle and Pathogenic Development. Plant Cell 22: 2908-2922.

Heimel, K., Scherer, M., Vranes, M., Wahl, R., Pothiratana, C., Schuler, D., et al. (2010b) The transcription factor Rbfl is the master regulator for $b$-mating type controlled pathogenic development in Ustilago maydis. PLoS Pathog 6: 17-18. 
Horst, R.J., Doehlemann, G., Wahl, R., Hofmann, J., Schmiedl, A., Kahmann, R., et al. (2010) Ustilago maydis Infection Strongly Alters Organic Nitrogen Allocation in Maize and Stimulates Productivity of Systemic Source Leaves ${ }^{[\mathrm{W}][\mathrm{OA}]}$. Plant Physiol 152: 293308.

Islamovic, E., García-Pedrajas, M.D., Chacko, N., Andrews, D.L., Covert, S.F., and Gold, S.E. (2015) Transcriptome Analysis of a Ustilago maydis ust1 Deletion Mutant Uncovers Involvement of Laccase and Polyketide Synthase Genes in Spore Development. Mol PlantMicrobe Interact 28: 42-54.

Jonkers, W., Rodriguez Estrada, A.E., Lee, K., Breakspear, A., May, G., and Kistler, H.C. (2012) Metabolome and Transcriptome of the Interaction between Ustilago maydis and Fusarium verticillioides In Vitro. Appl Environ Microbiol 3656-3667.

Kretschmer, M., Croll, D., and Kronstad, J.W. (2017a) Maize susceptibility to Ustilago maydis is influenced by genetic and chemical perturbation of carbohydrate allocation. Mol Plant Pathol 18: 1222-1237.

Kretschmer, M., Croll, D., and Kronstad, J.W. (2017b) Chloroplast-associated metabolic functions influence the susceptibility of maize to Ustilago maydis. Mol Plant Pathol 18: $1210-1221$.

Kretschmer, M., Klose, J., and Kronstad, J.W. (2012) Defects in Mitochondrial and Peroxisomal $\beta$-Oxidation Influence Virulence in the Maize Pathogen Ustilago maydis. Eukaryot Cell 11: 1055-1066.

Kretschmer, M., Lambie, S., Croll, D., and Kronstad, J.W. (2018) Acetate provokes mitochondrial stress and cell death in Ustilago maydis. Mol Microbiol 107: 488-507.

Lanver, D., Berndt, P., Tollot, M., Naik, V., Vranes, M., Warmann, T., et al. (2014) Plant Surface Cues Prime Ustilago maydis for Biotrophic Development. PLoS Pathog 10.

Lanver, D., Mendoza-Mendoza, A., Brachmann, A., and Kahmann, R. (2010) Sho1 and Msb2-Related Proteins Regulate Appressorium Development in the Smut Fungus Ustilago maydis . Plant Cell 22: 2085-2101.

Lanver, D., Müller, A.N., Happel, P., Schweizer, G., Haas, F.B., Franitza, M., et al. (2018) The Biotrophic Development of Ustilago maydis Studied by RNA-Seq Analysis. Plant Cell 30: $300-323$. 
Lanver, D., Tollot, M., Schweizer, G., Presti, L. Lo, Reissmann, S., Ma, L.S., et al. (2017) Ustilago maydis effectors and their impact on virulence. Nat Rev Microbiol 15: 409-421. León-Ramírez, C.G., Cabrera-Ponce, J.L., Martínez-Soto, D., Sánchez-Arreguin, A., Arechiga-Carvajal, E.T., and Ruiz-Herrera, R. (2017) Transcriptomic analysis of basidiocarp development in Ustilago maydis (DC) Cda. Fungal Biol Rev 101: 34-45.

Ma, L., Wang, L., Trippel, C., Mendoza-Mendoza, A., Ullmann, S., Moretti, M., et al. (2018) The Ustilago maydis repetitive effector Rsp3 blocks the antifungal activity of mannose-binding maize proteins. Nat Commun. 1-15.

Martínez-Soto, D., and Ruiz-Herrera, J. (2013) Transcriptomic analysis of the dimorphic transition of Ustilago maydis induced in vitro by a change in $\mathrm{pH}$. Fungal Genet Biol 5859: $116-125$.

Matei, A., Ernst, C., Markus, G., Thiele, B., Altmüller, J., Walbot, V., et al. (2018) How to make a tumour: cell type specific dissection of Ustilago maydis-induced tumour development in maize leaves. New Phytol 217: 1681-1695.

Morrison, E.N., Donaldson, M.E., and Saville, B.J. (2012) Identification and analysis of genes expressed in the Ustilago maydis dikaryon: Uncovering a novel class of pathogenesis genes. Can J Plant Pathol 34: 417-435.

Paul, J.A., Wallen, R.M., Zhao, C., Shi, T., and Perlin, M.H. (2018) Coordinate regulation of Ustilago maydis ammonium transporters and genes involved in mating and pathogenicity*. Fungal Biol 1-12.

Redkar, A., Hoser, R., Schilling, L., Zechmann, B., Krzymowska, M., Walbot, V., and Doehlemann, G. (2015) A Secreted Effector Protein of Ustilago maydis Guides Maize Leaf Cells to Form Tumors. Plant Cell 27: 1332-1351.

Sánchez-Arreguin, J.A., Hernandez-Onate, M.A., León-Ramirez, C.G., and Ruiz-Herrera, J. (2017) Transcriptional analysis of the adaptation of Ustilago maydis during growth under nitrogen fixation conditions. J Basic Microbiol 1-8.

Schilling, L., Matei, A., Redkar, A., Walbot, V., and Doehlemann, G. (2014) Virulence of the maize smut Ustilago maydis is shaped by organ-specific effectors. Mol Plant Pathol 15: $780-789$. 
Schuler, D., Wahl, R., Wippel, K., Vranes, M., Münsterkötter, M., Sauer, N., and Kämper, J. (2015) Hxt1, a monosaccharide transporter and sensor required for virulence of the maize pathogen Ustilago maydis. New Phytol 206: 1086-1100.

Skibbe, S.D., Doehlemann, G., Fernandes, J., and Walbot, V. (2010) Maize Tumors Caused by Ustilago maydis Require Organ-Specific Genes in Host and Pathogen. Science 67: 8993.

Tollot, M., Assmann, D., Becker, C., Altmüller, J., Dutheil, J.Y., Wegner, C.-E., and Kahmann, R. (2016) The WOPR Protein Ros1 Is a Master Regulator of Sporogenesis and Late Effector Gene Expression in the Maize Pathogen Ustilago maydis. PLoS Pathog 137.

Wahl, R., Wippel, K., Goos, S., Kämper, J., and Sauer, N. (2010a) A novel high-affinity sucrose transporter is required for virulence of the plant pathogen Ustilago maydis. PLoS Biol 8: 1-11.

Wahl, R., Zahiri, A., and Kämper, J. (2010b) The Ustilago maydis b mating type locus controls hyphal proliferation and expression of secreted virulence factors in planta. Mol Microbiol 75: 208-220.

Zahiri, A., Heimel, K., Wahl, R., Rath, M., and Kämper, J. (2010) The Ustilago maydis Forkhead Transcription Factor Fox1 Is Involved in the Regulation of Genes Required for the Attenuation of Plant Defenses During Pathogenic Development . Mol Plant-Microbe Interact 23: 1118-1129 
3 Chapter 3 | UPR-dependent inhibition of mating type signaling

\section{Suppression of mating type signaling via the unfolded protein response is mediated by the dual specificity phosphatase Rok1}

Lara Schmitz ${ }^{1}$, Melina A. Schwier ${ }^{1}$, Kai Heimel ${ }^{1}$

${ }^{1}$ Georg-August-University-Göttingen, Institute for Microbiology and Genetics

Department of Molecular Microbiology and Genetics, Grisebachstr. 8

D-37077 Göttingen, Germany

\section{Author contribution:}

LS and KH designed the study. LS carried out the experiments. MAS performed Yeast-Two-Hybrid analysis. LS designed the figures. LS wrote and $\mathrm{KH}$ edited the manuscript. 
Chapter 3 | UPR-dependent inhibition of mating type signaling

\subsection{Abstract}

The basidiomycete Ustilago maydis depends on the unfolded protein response to maintain homeostasis of the endoplasmic reticulum (ER) during pathogenic development in planta. UPR activity is required for efficient secretion of effectors and suppression of the plant defense response. The UPR is specifically activated after plant penetration and connected to the $b$-mating type dependent signaling cascade that controls pathogenic development. Interaction between the UPR regulator Cib1 and the $b$-dependent regulator of development, Clp1, facilitates proliferation in planta. By contrast, premature UPR activation inhibits expression of the central regulators of pathogenic development, $b E, b W$ and $r b f 1$, thereby interfering with the formation of the infectious dikaryotic filament. We show that the UPR not only suppresses the $b$-regulatory network, but also interferes with the transcriptional and morphological response to pheromone. The MAPK Kpp2, which is part of the pheromone-responsive MAPK module, is dephosphorylated upon UPR activity, resulting in reduced activity of the central transcriptional regulator Prf1. Importantly, deletion of the dual specificity phosphatase rok1 restored Kpp2 phosphorylation. Since we observed that activity of the mating type pathway is suppressed by additional mechanisms, our study suggests that an extended regulatory crosstalk between the UPR and mating type pathways occurs during pathogenic growth inside the host plant.

\subsection{Introduction}

In fungal pathogens, crosstalk between signaling pathways is essential for accurate execution of developmental programs and adaption to the host environment. The biotrophic plant pathogen $U$. maydis is highly adapted to its host plant Zea mays (maize) and plant colonization is prerequisite for completion of its life cycle (Banuett, 1992). Pathogenic development depends on the formation of the filamentous dikaryon, which is formed upon fusion of two compatible cells and capable to infect the plant (Bölker et al., 1995). After plant penetration and establishment of a compatible biotrophic interaction, the fungus grows intracellular without disrupting the hosts plasma membrane, followed by extensive hyphal proliferation and tumor induction.

The initial stages of pathogenic development are controlled by the biallelic $a$-mating type locus. The $a$ locus encodes a pheromone ( $m f a) /$ receptor ( $p r a)$ system mediating cell-cell recognition and mating of two compatible haploid cells (Bölker et al., 1992). Perception of compatible pheromone triggers a G2 cell cycle arrest, conjugation tube formation and 
increased expression of pheromone-responsive genes. Signal transduction within the pheromone response is mediated by a cAMP-dependent Protein kinase A (PKA) and a Mitogen-activated protein kinase (MAPK) module. The MAPK module comprises the MAPKKK Kpp4 (Andrews et al., 2000; Müller et al., 2003), the MAPKK Fuz7 (Banuett and Herskowitz, 1994) and the terminal MAPK Kpp2 (Mayorga and Gold, 1999; Müller et al., 1999). Activity of Kpp2 controls conjugation tube formation and promotes phosphorylation of the pheromone response factor 1 (Prfl) (Müller et al., 2003). Prf1 belongs to the class of high-mobility-group (HMG) box proteins and regulates, depending on its phosphorylation status, transcription of the $a$ - and $b$-mating type genes (Urban et al., 1996; Kaffarnik et al., 2003; Zarnack et al., 2008).

After fusion of two compatible cells, the b-heterodimer, encoded by the multiallelic $b$-mating type locus, regulates all subsequent steps of sexual and pathogenic development. Transcriptional active, heterodimeric complexes are formed by the $b E$ and $b W$ homeodomain proteins only when derived from different alleles (Kämper et al., 1995). $\mathrm{bE} / \mathrm{bW}$-dependent gene regulation is orchestrated by a hierarchically transcription factor network that controls expression of approximately 340 genes (Heimel et al., 2010b). Activation of this pathway facilitates maintenance of the cell cycle arrest, filamentous growth and penetration of the plant surface (Schulz et al., 1990; Kämper et al., 1995). Direct targets of the b-heterodimer include $c l p 1$, the central regular of fungal development in planta, and rbf1 (Heimel et al., 2010a; Heimel et al., 2010b). Rbf1 is a C2H2 zinc finger transcription factor and key regulator downstream of the $\mathrm{bE} / \mathrm{bW}$ heterodimer, it is required for regulation of more than $90 \%$ of the b-dependent genes (Heimel et al., 2010b). Consequently, Rbfl is required and sufficient to induce filamentous growth, maintain the G2 cell cycle arrest, appressoria formation and plant penetration. Only after successful plant penetration, the cell cycle arrest is released and proliferation and mitotic division of the dikaryotic filament is initiated. This developmental switch is controlled by the Clp1 protein that is post-transcriptionally regulated and specifically accumulates after plant penetration (Scherer et al., 2006; Heimel et al., 2010a). Clp1 mediates the release from the cell cycle block via physical interaction with bW and Rbf1, inhibiting the function of the b-heterodimer and the pheromone response pathway, respectively. Hence, establishment of a negative feedback loop leads to quenching of the $a$ - and $b$-mating type pathways, releasing the cell cycle block and inducing fungal proliferation in planta. 
Chapter 3 | UPR-dependent inhibition of mating type signaling

To establish a compatible biotrophic interaction between $U$. maydis and its host plant maize, the fungus secrets a large number effector proteins (Lo Presti et al., 2015a; Lanver et al., 2017). The concerted upregulation of effector gene expression results in increased stress on the endoplasmic reticulum (ER) that is counteracted by the unfolded protein response (UPR). The UPR is a highly conserved eukaryotic signaling pathway to maintain ER homeostasis by increasing the ER folding capacity and increased degradation of terminally misfolded and potentially toxic proteins. The ER-membrane localized RNase/kinase Ire1p is activated upon sensing of un- or misfolded proteins, leading to unconventional cytoplasmic splicing of the mRNA encoding the central transcriptional regulator of the UPR termed Hac1p in yeast, XBP1 in mammals and Cib1 in U. maydis (Ron and Walter, 2007; Walter and Ron, 2011; Heimel et al., 2013). Ire1-dependent removal of the unconventional intron promotes expression of the Hac1-like transcriptional activator, thereby regulating UPR target gene expression (Cox et al., 1997; Kawahara et al., 1998; Rüegsegger et al., 2001). Although the overall UPR is protective, prolonged or hyper-activation of the UPR is deleterious for the cell and can lead to UPR-induced cell death (Shore et al., 2011; Tabas and Ron, 2011; Hetz, 2012).

The UPR-mediated ER stress resistance is critical for virulence of pathogenic fungi. In U. maydis, the UPR is specifically activated after plant penetration and coordinates biotrophic growth in planta (Heimel et al., 2013). Upon physical interaction between the UPR regulator $\mathrm{Cib} 1$ and the $\mathrm{Clp} 1$ protein, Clp1 accumulates and initiates fungal proliferation in planta. By contrast, premature UPR activation interferes with the formation of $b$-dependent filaments and expression of $b E, b W$ and $r b f 1$ genes. This inhibitory effect is independent of Clp1, suggesting that additional crosstalk between the UPR and mating type pathways exist in $U$. maydis.

Here, we show that an active UPR interferes with the mating type-dependent signaling cascade on multiple levels. Constitutive activation of the UPR inhibits the morphological and transcriptional response of haploid cells towards pheromone stimulus. We demonstrate that crosstalk between the UPR and the pheromone response pathway leads to almost abolished phosphorylation of the MAPK Kpp2. Phosphorylation can be rescued by deleting the dual specificity phosphatase rokl. Since both pathways are active in planta, and deletion of rokl results in hypervirulence, our results suggest that the UPR effectively antagonizes mating type dependent signaling as a mechanism to maintain biotrophy. 


\subsection{Results}

\subsubsection{UPR activity inhibits $b$-mating type dependent regulatory network}

In $U$. maydis, the $\mathrm{bE} / \mathrm{bW}$-heterodimer is the central regulator of pathogenic development (Schulz et al., 1990; Gillissen et al., 1992; Kämper et al., 1995; Heimel et al., 2010b). In the solopathogenic strain SG200, expression of the compatible $b E 1 / b W 2$ genes supersedes the need of a mating partner to trigger the pathogenic program (Kämper et al., 2006). UPR activity is regulated by unconventional splicing of the cibl mRNA, encoding the central regulator of the UPR in U. maydis. To study the effects of a constitutive active UPR on pathogenic development, the intronless $c i b 1^{\mathrm{s}}$ mRNA under control of its endogenous promoter was integrated into the ip locus of strain SG200 in single $\left(c i b 1^{\mathrm{s}}\right)$ or multiple copies $\left(c i b 1^{\mathrm{s}(\mathrm{x})}\right)\left(\right.$ Heimel et al., 2013). Expression of $c i b 1^{\mathrm{s}}$ is sufficient to activate the UPR in an ER-stress independent manner. Previous studies demonstrated that filament formation of SG200 is suppressed by an active UPR by a dose-dependent reduction of $b E, b W$ and $r b f 1$ transcript levels (Figure 3.1A) (Heimel et al., 2013). Since the b-regulatory network is composed of hierarchically arranged transcription factors, we determined expression levels of the key transcriptional regulators Biz1, Hdp1 and Hdp2 (Flor-Parra et al., 2006; Heimel et al., 2010b; Lanver et al., 2014) downstream of Rbf1 by quantitative reverse transcriptase (qRT)-PCR analysis. Consistent with the results obtained for the $b$-genes and $r b f 1$, expression levels of all three transcription factors were significantly and dose-dependently reduced by $c i b 1^{\text {s }}$ (Figure 3.1B). This suggests that UPR activity leads to the suppression of the complete b-regulatory network.

To address at which stage the UPR interferes with the mating type signaling pathway, we investigated the effects of UPR activity on the pheromone response pathway acting upstream of $\mathrm{bE} / \mathrm{bW}$. To this end, we determined expression levels of $m f a 1$, pral (encoding the pheromone precursor and the pheromone receptor, respectively) and of prfl in SG200 (WT control) and the $c i b 1^{\mathrm{s}}$-expressing derivative. In comparison to SG200, expression of all three genes was significantly reduced in $c i b l^{\text {s}}$-expressing strains (Figure 3.1C). Moreover, expression of two transcriptional regulators of prf1, hap2 and rop1, and of additional Prf1 target genes was suppressed by the UPR (Zarnack et al., 2008) (Figure 3.1D). Interestingly, UPR activity suppressed Prf1-dependent expression (UMAG_00306, $U M A G \_05348$ and $\left.U M A G \_06190\right)$ as well as repression (UMAG_10838) of target genes (Figure 3.1D). This suggests that the negative effect of the UPR leads to reduced Prfl activity and interferes with mating type signaling upstream of the b-heterodimer. 
A

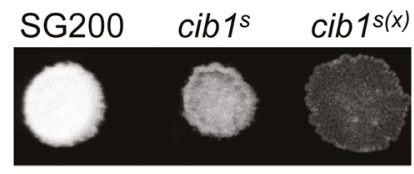

C

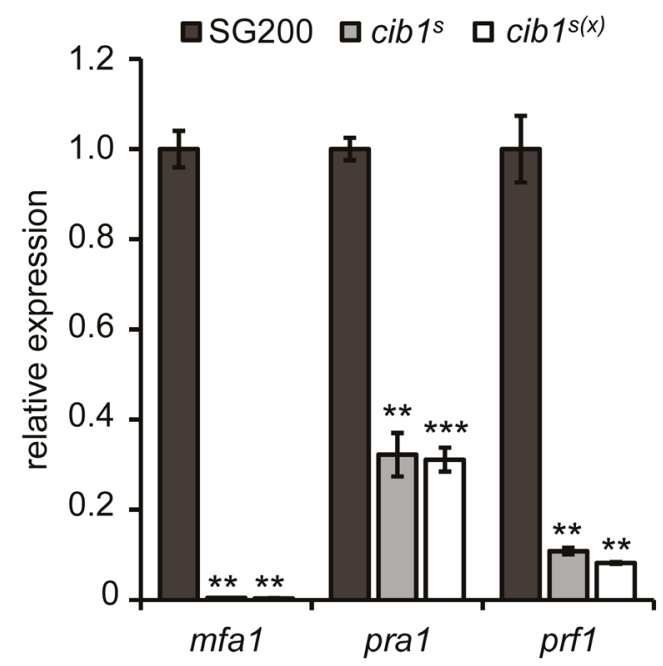

B

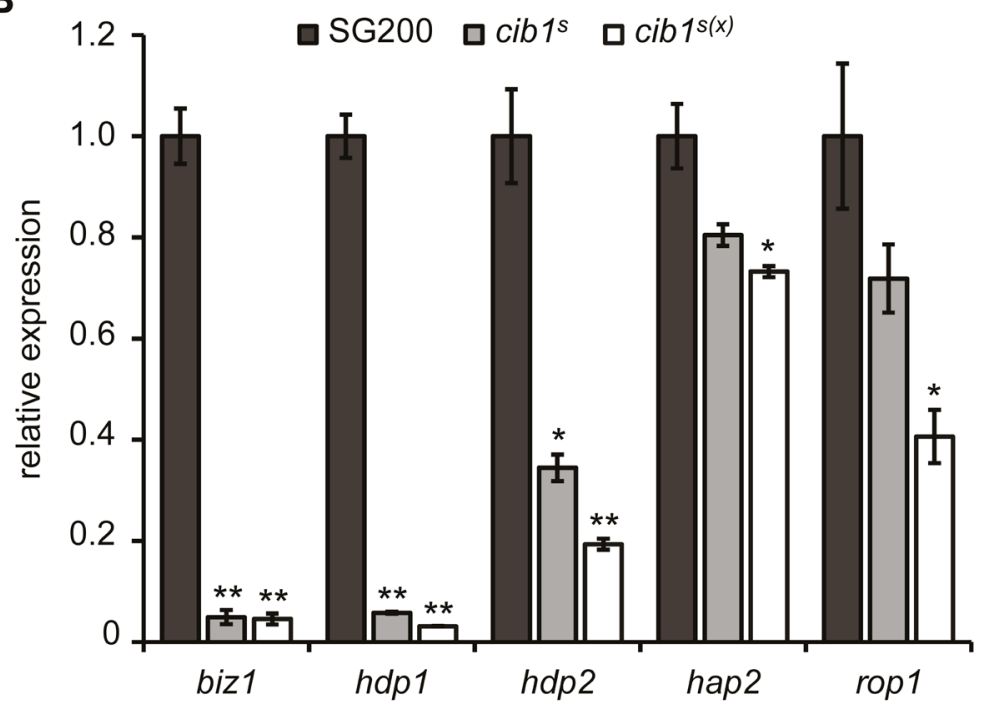

D

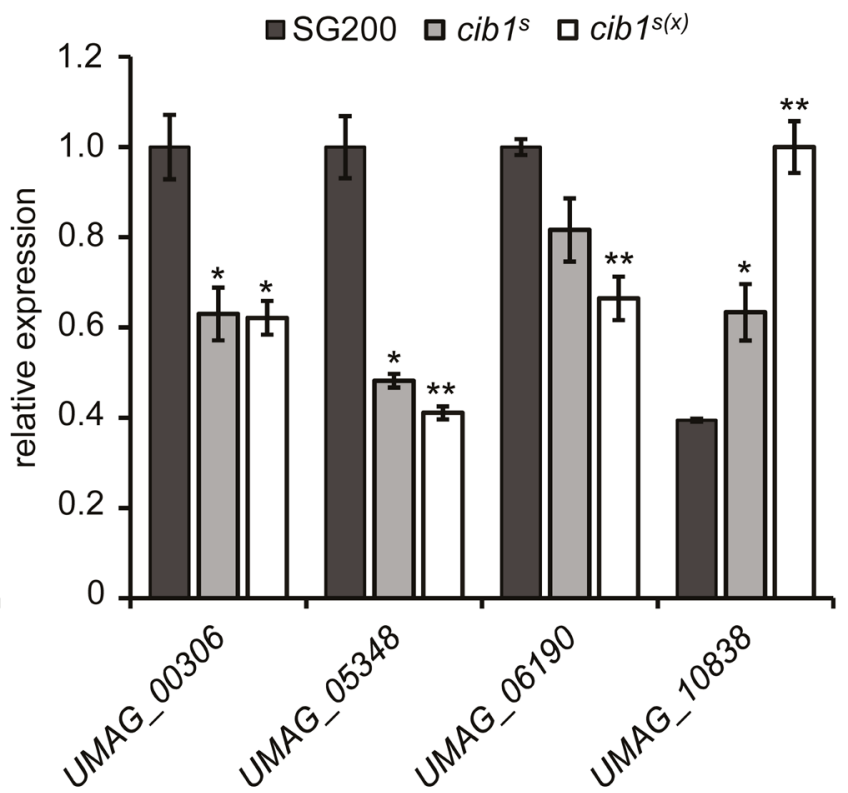

Figure 3.1 UPR suppresses b-dependent filament formation and gene expression. (A) Analysis of $b$-dependent filament formation. SG200 and derivatives constitutively expressing $c i b 1^{\mathrm{s}}$ or $c i b 1^{\mathrm{s}(\mathrm{x})}$ were spotted on potato dextrose solid media supplemented with $1 \%$ charcoal. Photos were taken after 24 hours at $28^{\circ} \mathrm{C}$. White fuzzy colonies indicate the formation of filaments. (B-D) qRT-PCR analysis of gene expression in SG200 and derivatives expressing one $\left(c i b 1^{\mathrm{s}}\right)$ or multiple copies $\left(c i b 1^{\mathrm{s}(\mathrm{x})}\right)$ of $c i b 1^{\mathrm{s}}$. elF2b was used for normalization. Expression values represent the mean of three biological replicates with two technical duplicates each. Error bars represent the SEM. Statistical significance was calculated using the students $t$ test. $* \mathrm{P}$ value $<0.05, * * \mathrm{P}<0.01$, and $* * * \mathrm{P}<0.001$ (B) Expression of $r b f 1$-regulated transcription factors bizl, $h d p 1$ and $h d p 2$ and regulators of prf1 expression, rop1 and hap2. (C) Expression of the pheromone genes mfal, pral and the central transcription factor prfl. (D) Expression values of genes UMAG_00306, $U M A G_{-} 05348, \quad U M A G_{-} 06190$ and $U M A G_{-} 10838$, which are differentially expressed upon MAPK-dependent phosphorylation of Prf1. 


\subsubsection{UPR represses morphological and transcriptional response to pheromone}

The solopathogenic strain SG200 expresses a compatible pheromone/pheromone receptor pair and compatible $b$-genes, leading to constitutive activation of $a$ - and $b$-mating type pathways. Both pathways are regulated and connected by various feedback loops (Urban et al., 1996; Zarnack et al., 2008; Heimel et al., 2010a). Similar to the results obtained in the SG200 background, mating assays between compatible FB1 and FB2 strains revealed reduced filament formation in $c i b l^{\text {s}}$-expressing strains on charcoal containing solid media (Suppl. Figure 3.3). To specifically address the effect of the UPR on the pheromone response (controlled by the a-mating type locus), we treated liquid cultures of FB1 (WT control) and $c i b 1^{\mathrm{s}}$-expressing derivatives with synthetic $a 2$ pheromone and quantified conjugation tube formation. This revealed that conjugation tube formation was reduced in $c i b 1^{\mathrm{s}}$-expressing strains when compared to FB1 (Figure 3.2A). In FB1, 32.9\% of the cells formed conjugation tubes $6 \mathrm{~h}$ after treatment with compatible $a 2$ pheromone $(2.5 \mu \mathrm{g} / \mathrm{mL})$. By contrast, only $17.3 \%$ and $4.5 \%$ of the cells formed conjugation tubes in FB1 $\mathrm{cibl}^{\mathrm{s}}$ and FB1 $c i b 1^{\mathrm{s}(\mathrm{x})}$ strains under identical conditions (Figure 3.2B). This demonstrates that the UPR inhibits the morphological response to pheromone in a dose-dependent manner.

We next tested if suppression of the pheromone-induced morphological response correlates with an altered transcriptional response. To this end, expression levels of the $a$-mating type genes $m f a l$ and pral and of prfl in pheromone treated FB1 (WT) and $\mathrm{cibl}^{\mathrm{s}}$-expressing derivatives were determined by qRT-PCR. This revealed that basal expression levels of all three genes were significantly reduced in the $c i b l^{\mathrm{s}}$-expressing strain compared to FB1. Pheromone treatment resulted in strongly increased transcript levels of $m f a l$, pral and prfl in FB1, whereas gene induction in the $c i b 1^{\mathrm{s}}$-expressing strain was only slightly increased and approximately 5-fold lower than in FB1 (Figure 3.2C). This suggests that an active UPR inhibits both, the morphological and the transcriptional response to pheromone.

Since signal transduction within the pheromone response is mediated by PKA- and MAPK-pathways, we first tested whether UPR activation affects the activity of the cAMP-dependent PKA pathway. To specifically activate the PKA pathway, we treated FB1 (WT control) and FB1 $\mathrm{cibl}^{\mathrm{s}}$ cells with $6 \mathrm{mM}$ cAMP and monitored expression of $m f a 1$ and pral. This revealed that cAMP-induced expression of $m f a l$ and pral was lower in $c i b 1^{\mathrm{s}}$ expressing strains when compared to FB1 (Figure 3.2D). However, the inhibitory effect of an active UPR after cAMP treatment was only modest and less pronounced when compared 
to pheromone treated cells. Therefore, the strong impact of an active UPR on the pheromone response pathway is most likely achieved via the MAPK signaling pathway.

A

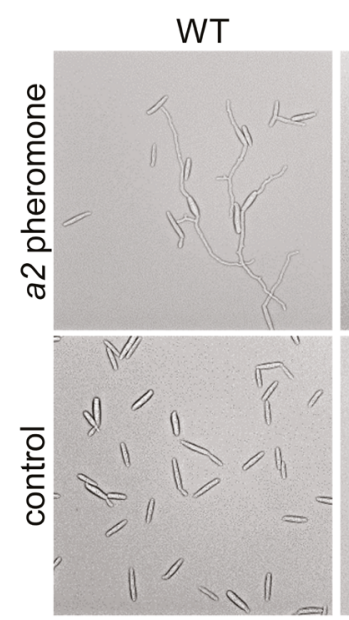

C

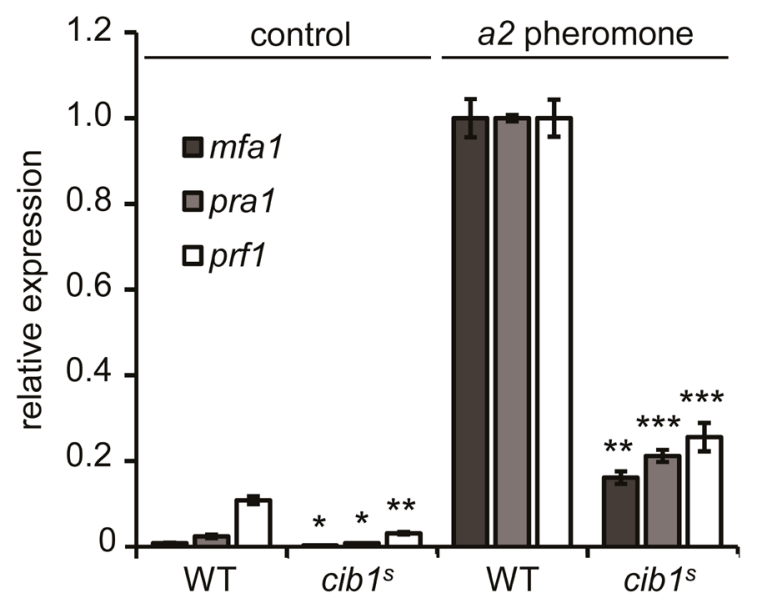

B

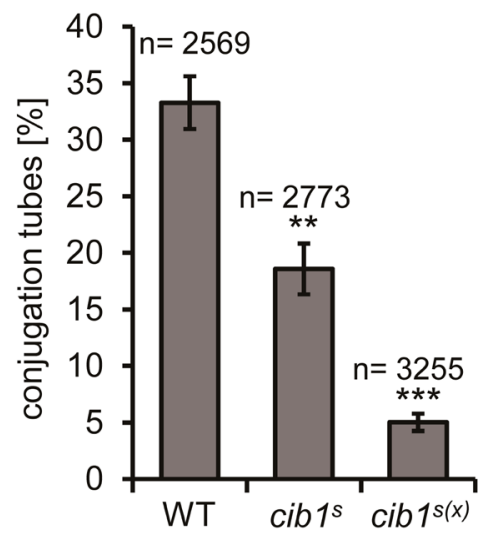

Figure 3.2 The morphological and transcriptional response to pheromone is inhibited by UPR activity. (A) Microscopic analysis of pheromone-induced conjugation tube formation. Wild type strain FB1 (WT) and derivatives constitutively expressing $c i b 1^{\mathrm{s}}$ or $c i b 1^{\mathrm{s}(\mathrm{x})}$ were treated with synthetic $a 2$-pheromone or DMSO as negative control and grown for 6 hours at $28^{\circ} \mathrm{C}$ under rotation. Formation of conjugation tubes was monitored by bright field microscopy. Bar $=10 \mu \mathrm{m}$. (B) Quantification of conjugation tube formation. The bars represent mean value of three independent experiments. Error bars represent the SD. $n=$ number of total cells counted. Statistical significance was calculated using the students $t$ test. ${ }^{* *} \mathrm{P}$ value $<0.01$, and $* * * \mathrm{P}<0.001$. (C) qRTPCR analysis of $m f a 1$, pral and prfl gene expression in FB1 (WT) and derivatives expressing $c i b 1^{\mathrm{s}}$ after treatment with synthetic $a 2$ pheromone. Cells were treated with synthetic $a 2$ pheromone for 6 hours at $28^{\circ} \mathrm{C}$. $e I F 2 b$ was used for normalization. Expression values represent the mean of three biological replicates with two technical duplicates each. Error bars represent the SEM. Statistical significance was calculated using the students $t$ test. $* \mathrm{P}$ value $<0.05,{ }^{*} \mathrm{P}<0.01$, and $* * * \mathrm{P}<0.001$. (D) qRT-PCR analysis of genes $m f a 1$ and pra 1 in FB1 (WT) and derivatives expressing $c i b 1^{\mathrm{s}}$ after treatment with cAMP. Cells were treated with $6 \mathrm{mM}$ cAMP for 12 hours at $28^{\circ} \mathrm{C}$. $e I F 2 b$ was used for normalization. Expression values represent the mean of two biological replicates with two technical duplicates each. Error bars represent the SEM. 


\subsubsection{Induced expression of $f u z^{D D}$ rescues the morphological but not the transcriptional UPR-dependent inhibition of pheromone signaling}

To address at which stage the UPR and pheromone pathway interact, we expressed the active MAPK kinase $f u z 7^{D D}$ under control of the arabinose-inducible $\mathrm{crg}$ promoter in FB1 and FB1 $\mathrm{cibl}^{\mathrm{s}}$ strains (Brachmann et al., 2001; Müller et al., 2003). Arabinose-induced expression of $f u z 7^{D D}$ leads to genetic activation of the MAPK pathway independent of pheromone sensing and results in formation of filamentous cells, resembling conjugation tubes (Müller et al., 2003). Induced expression of $f u z 7^{D D}$ triggered formation of conjugation tube-like structures in $86.8 \%$ of the FB1 cells and in $95.9 \%$ of $c i b 1^{\mathrm{s}}$ cells (Figure 3.3A and B). Conversely, transcript levels of the pheromone genes $m f a 1$, pral and of prfl were highly induced by $f u z 7^{D D}$ in FB1 but significantly lower (3-fold) when $c i b 1^{\text {s }}$ was expressed (Figure 3.3C). Hence, the UPR inhibits the Fuz $7^{\mathrm{DD}}$-induced gene regulation but not the morphological response. Interestingly, the transcriptional regulation of the $\mathrm{mfal}$ and pral genes is Prfl-dependent, whereas the morphological response is not (Müller et al., 2003; Brefort et al., 2005). This indicates that the UPR negatively affects Prf1 activity, which is regulated by phosphorylation of the MAPK Kpp2. 
A

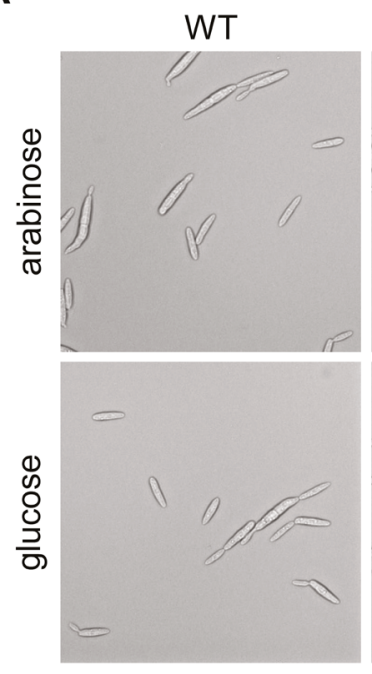

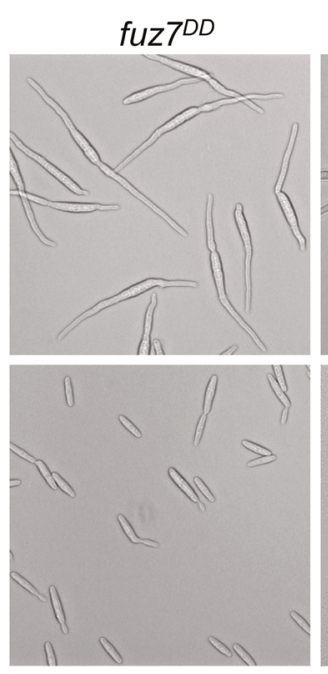

B

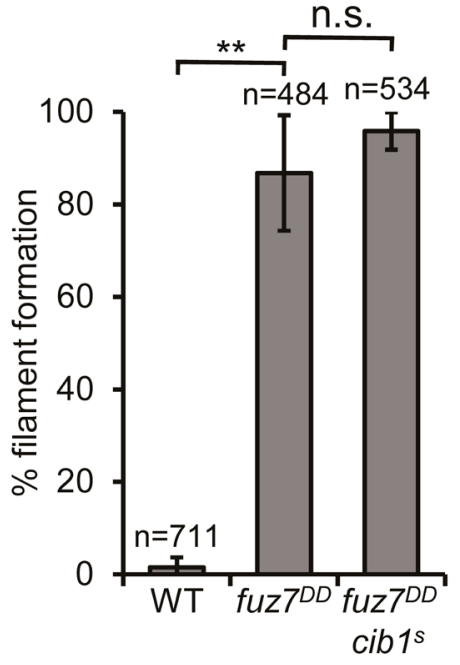

C

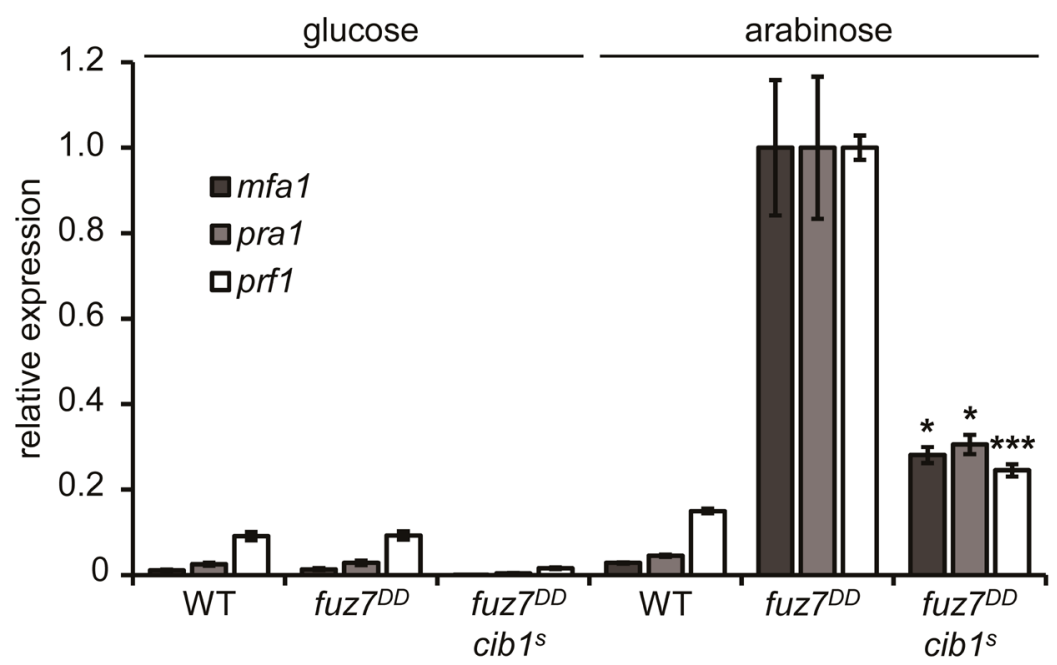

Figure 3.3 The UPR differentially affects $\mathrm{Fuz}^{\mathrm{DD}}$-induced morphological and transcriptional responses. (A) Microscopic analysis of $f u z 7^{D D}$-induced conjugation tube-like structures. Strain FB1 (WT), FB1 $f u z 7^{D D}$ ( $f u z 7^{D D}$ expression under control of the arabinose-inducible $\operatorname{crg} 1$ promoter) and a derivative expressing $\mathrm{cib} 1^{\mathrm{s}}$ were grown for 6 hours at $28^{\circ} \mathrm{C}$ in $\mathrm{CM}$-glucose (non-inducing) or $\mathrm{CM}$-arabinose (inducing) medium. Formation of conjugation tube-like structures was monitored by bright field microscopy. Bar $=10 \mu \mathrm{m}$. (B) Quantification of formation of conjugation tube-like structures. Cells were counted based on microscopic pictures and number of cells forming conjugation tubes was determined. The bars represent mean value of three independent experiments. Error bars represent the SD. $n=$ number of total cells counted. Statistical significance was calculated using the students $t$ test. $* *$ P value $<0.01$. (C) qRT-PCR analysis of $m f a 1$, pral and prf1 gene expression in FB1 (WT), FB1 $f u z 7^{D D}$ ( $f u z 7^{D D}$ expression under control of the arabinoseinducible $\mathrm{crg} 1$ promoter) and a derivative expressing $c i b 1^{\text {s }}$. Cells were grown for 6 hours at $28^{\circ} \mathrm{C}$ in $\mathrm{CM}$ glucose (non-inducing) or CM-arabinose (inducing) medium. $e I F 2 b$ was used for normalization. Expression values represent the mean of three biological replicates with two technical duplicates each. Error bars represent the SEM. Statistical significance was calculated using the students $t$ test. $* \mathrm{P}$ value $<0.05$, and $* * * \mathrm{P}$ $<0.001$. 


\subsubsection{Phosphorylation of Kpp2 is suppressed by the UPR}

To address if the UPR affects the MAPK cascade at the level of Kpp2, we expressed a functional Kpp2-GFP fusion protein under the control of its endogenous promoter from the endogenous genomic locus in WT $\left(f u z 7^{D D}\right)$ and $c i b 1^{\mathrm{s}}$-expressing $\left(f u z 7^{D D} c i b 1^{\mathrm{s}}\right)$ strains. Western hybridization revealed that abundance of Kpp2-GFP was not altered by $f u z 7^{D D}$ or $c i b 1^{\mathrm{s}}$ expression in all tested strains and conditions (Figure 3.4A). By contrast, phosphorylation of the Kpp2-GFP fusion protein was strongly increased in response to induced expression of $f u z 7^{D D}$ compared to the non-induced control. Importantly, this effect was almost completely abolished in the $c i b 1^{\mathrm{s}}$-expressing strains under identical conditions (Figure 3.4A). Since Fuz $7^{\mathrm{DD}}$-mediated activation of the MAPK pathway relies on an artificial expression system, we monitored the effect of an active UPR on Kpp2 phosphorylation in response to pheromone treatment. Consistent with the results obtained using $f u z 7^{D D}$-expressing strains, expression of $c i b 1^{\mathrm{s}}$ did not affect abundance of Kpp2-GFP but resulted in strongly reduced phosphorylation of Kpp2-GFP (Figure 3.4B). This indicates that the UPR negatively affects phosphorylation and thereby activity of Kpp2.

A

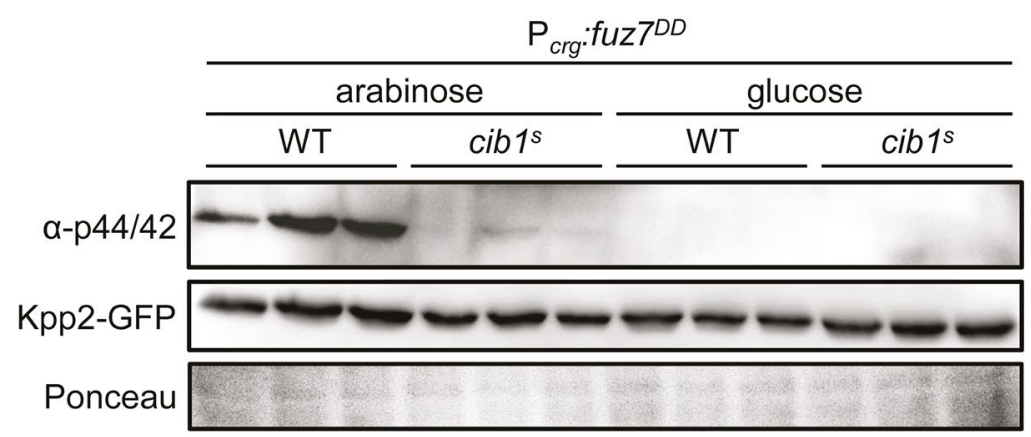

B

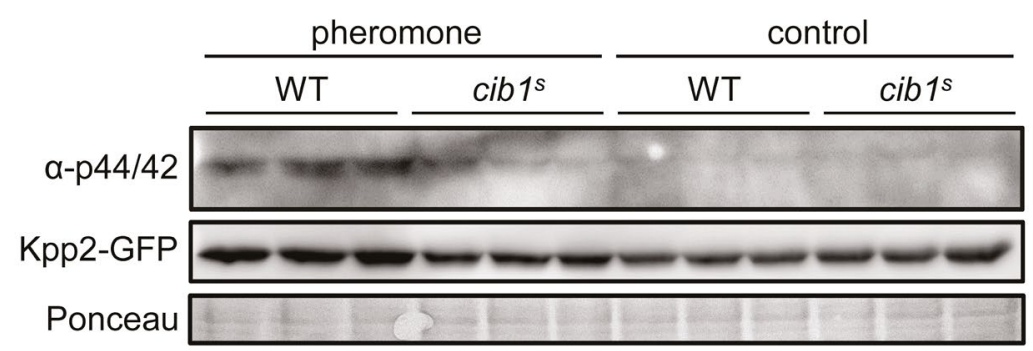

Figure 3.4 Expression of cibl $^{\text {s }}$ diminishes Kpp2 phosphorylation. (A) Immunoblot analysis of Kpp2-GFP protein levels and phosphorylation. Derivatives of strains FB1 $f u z 7^{D D}\left(f u z 7^{D D}\right.$ expression under control of the arabinose-inducible $\mathrm{crg} 1$ promoter, WT) and FB1 $\mathrm{fuz}^{D D} \mathrm{cibl}^{\mathrm{s}}\left(\mathrm{cibl}^{\mathrm{s}}\right)$ expressing a Kpp2-GFP fusion protein were grown in CM-glucose (non-inducing) or CM-arabinose (inducing) liquid medium for 6 hours at $28^{\circ} \mathrm{C}$. Kpp2-GFP was visualized using a GFP-specific antibody. Phosphorylation of Kpp2-GFP was visualized using the $\alpha$-phospho44/42 antibody that recognizes the phosphorylated TEY motif of MAPKs. The Ponceau stained membrane served as loading control. (B) Immunoblot analysis with the same strains as in (A) treated with synthetic $a 2$ pheromone for 6 hours at $28^{\circ} \mathrm{C}$. The same antibodies as in (A) were used to visualize Kpp2-GFP and MAPK phosphorylation. The Ponceau stained membrane served as loading control. 
Chapter 3 | UPR-dependent inhibition of mating type signaling

\subsubsection{UPR-dependent suppression of Kpp2 phosphorylation depends on the dual specificity phosphatase (DSP) Rok1}

The physical interaction between MAPK-pathway components is prerequisite for signal transduction within MAPK cascades and regulated by scaffold proteins to establish signaling modules and foster efficient phospho-transfer between interacting proteins (Choi et al., 1994; Dard and Peter, 2006). To analyze if the UPR affects the interaction between Fuz7 and Kpp2, we expressed Kpp2-GFP and Fuz ${ }^{\mathrm{DD}}$-HA fusion proteins in FB1 (WT control) and $c i b l^{\mathrm{s}}$ expressing strains. Cells were grown under $f u z 7^{D D}$-inducing conditions and immunoprecipitation of Kpp2-GFP was performed using GFP-trap beads (Chromotek). Co-immunoprecipitation of Fuz $7^{\mathrm{DD}}$-HA was tested using an HA-specific antibody (Sigma). This revealed that Fuz $7^{\mathrm{DD}}$-HA was co-precipitated in similar amounts in the WT and $c i b 1^{\mathrm{s}}$ expressing strain. By contrast, Fuz $7^{\mathrm{DD}}$-HA was not co-immunoprecipitated in the control strain expressing free GFP instead of Kpp2-GFP (Figure 3.5A). This indicates that Kpp2 and Fuz7 interaction is specific and not affected by UPR activity.

Activity of MAPKs is regulated by relative activities of phosphorylating and dephosphorylating proteins. Previously, it has been shown that the DSP Rok1 mediates dephosphorylation of Kpp2 and thereby counteracts activity of pheromone response pathway (Di Stasio et al., 2009). To test if the inhibitory effect of an active UPR is mediated by Rok1, we generated rokl deletion strains in the WT (FB1 fuz $7^{D D}$ ) and the $c i b 1^{\mathrm{s}}$ expressing derivative (FB1 $f u z 7^{D D} c i b 1^{\mathrm{s}}$ ). Consistent with our previous results, Kpp2-GFP abundance was not altered under any of the conditions tested. Strikingly, induced expression of $f u z 7^{D D}$ resulted in Kpp2 phosphorylation in both, WT and $c i b 1^{\mathrm{s}}$ expressing $\Delta r o k 1$ cells (Figure 3.5B). Hence, deletion of rokl fully repressed the negative effect of an active UPR on Kpp2 phosphorylation.

To test how the UPR regulates Rok1, we compared Rok1 transcript and protein levels between WT and $c i b l^{s}$-expressing strains. rokl gene expression is approximately 2-fold increased by $f u z 7^{D D}$ in both strains (Figure 3.5C), but only slightly reduced in $c i b 1^{\mathrm{s}}$-expressing strains, when compared to the WT control. This indicates that the UPR affects Rok1 in a posttranscriptional manner. To test for potential UPR-induced alterations of Rok1 protein levels, we expressed a Rok1-mCherry fusion protein under control of the endogenous promoter from the endogenous genomic locus in WT and $c i b 1^{\mathrm{s}}$-expressing strains. Since the fusion protein was not detectable by Western hybridization, Rok1-mCherry was enriched by immunoprecipitation. Total protein amounts of $U$. maydis protein extracts were adjusted and immunoprecipitation was performed with a higher 
volume of mCherry-Trap beads (Chromotek) to prevent saturation of the beads and enable a quantitative comparison between both strains. While Rok1-mCherry was not detectable under non-induced conditions, protein levels were similar in both strains in response to $f u z 7^{D D}$-mediated genetic activation of the MAPK pathway (Figure 3.5D). Taken together, these results suggest that the UPR regulates Rok1 on posttranslational level, potentially by influencing Rok1 activity.

Surprisingly, in contrast to the restored Kpp2 phosphorylation in rok1 deletion strains, expression levels of $m f a l$, pral and of $p r f l$ were still $c i b l^{\text {s}}$-dependently suppressed. Hence, phosphorylation of Kpp2 is not sufficient for $m f a 1$, pral and prfl expression under these conditions (Suppl. Figure 3.3). In conclusion, this suggests that the UPR interferes with expression of Prf1 target genes on multiple levels. 
Chapter 3 | UPR-dependent inhibition of mating type signaling

A

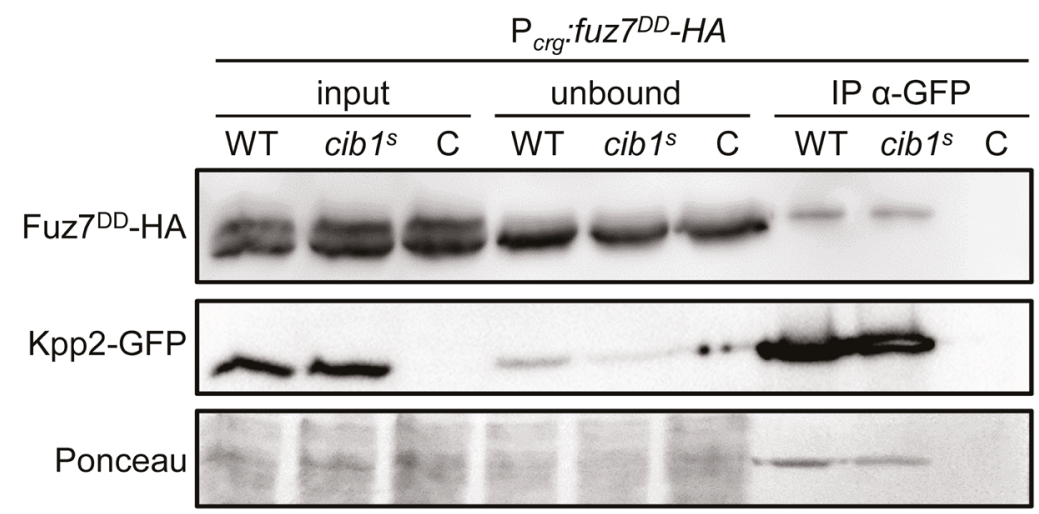

B

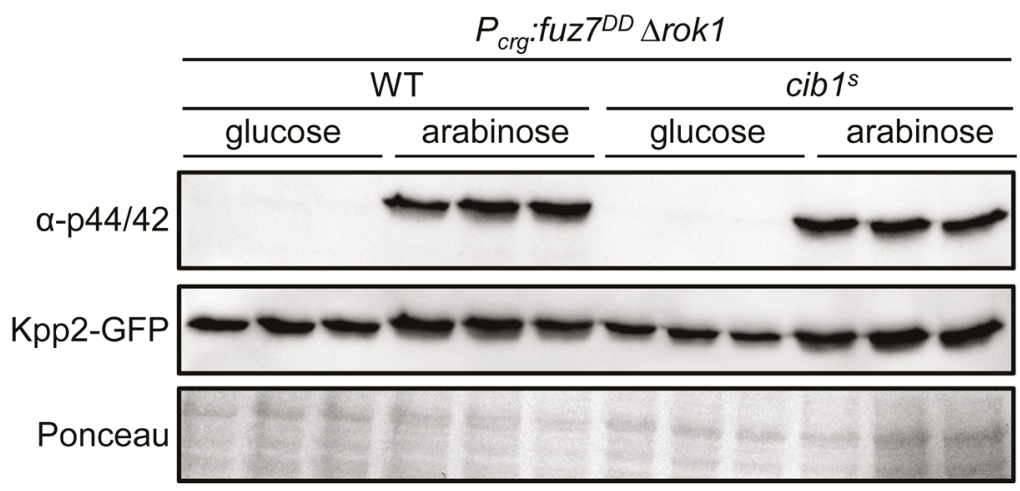

C

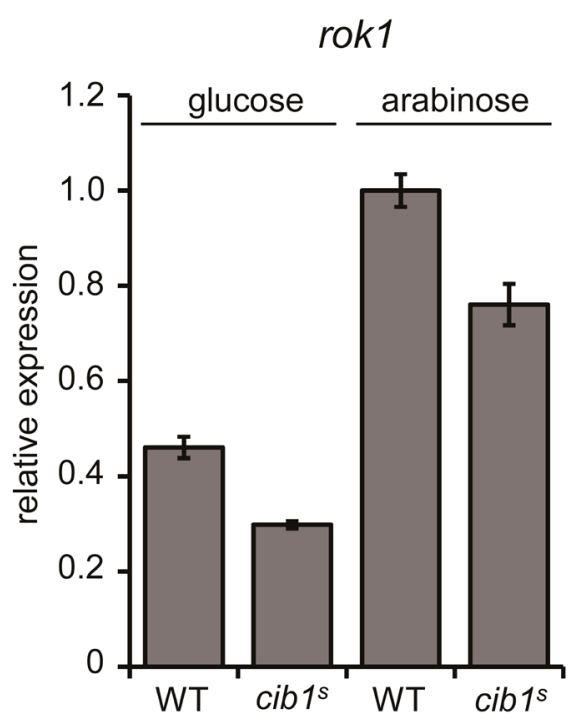

D

IP a-mCherry

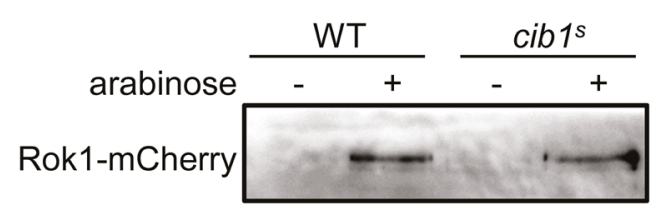


Figure 3.5 Dephosphorylation of Kpp2 upon cib1 ${ }^{\text {s }}$ expression depends on the DPS Rok1. (A) Co-immunoprecipitation analysis of Kpp2-GFP and Fuz $7^{D D}$-HA. Derivatives of strains FB1 fuz $7^{D D}$ $k p p 2-G F P(\mathrm{WT})$ and FB1 $f u z 7^{D D} k p p 2-G F P c i b 1^{\mathrm{s}}\left(c i b 1^{\mathrm{s}}\right)$ containing a Fuz $7^{\mathrm{DD}}-\mathrm{HA}$ fusion protein under control of the arabinose-inducible $\mathrm{crg} 1$ promoter were grown in CM-arabinose (inducing) liquid medium for 6 hours at $28^{\circ} \mathrm{C}$. A strain expressing untagged Kpp2 served as negative control. Total protein extract was subjected to immunoprecipitation using GFP-specific beads. Co-immunoprecipitation of Fuz $7^{\mathrm{DD}}$-HA was visualized using an HA-specific antibody. Kpp2-GFP was visualized using an GFP-specific antibody. The Ponceau stained membrane served as loading control. (B) Immunoblot analysis of Kpp2-GFP protein levels and phosphorylation in rokl deletion strains. Derivatives of strains FB1 $f u z 7^{D D}$ (WT) and FB1 $f u z 7^{D D} c i b 1^{\mathrm{s}}$ $\left(c i b 1^{\mathrm{s}}\right)$ deleted for rokl were grown in CM-glucose (non-inducing) or CM-arabinose (inducing) liquid medium for 6 hours at $28^{\circ} \mathrm{C}$. Cells were subjected to immunoblot analysis, Kpp2-GFP levels were visualized using a GFP-specific antibody. Phosphorylation of Kpp2-GFP was visualized using the $\alpha$-phospho44/42 antibody that specifically recognizes the phosphorylated TEY motif of MAPKs. The Ponceau stained membrane served as loading control. (C) qRT-PCR analysis of rokl gene expression in FB1 $f u z 7^{D D}$ (WT) and a derivative expressing cibl ${ }^{\mathrm{s}}$. Cells were grown for 6 hours in CM-glucose (non-inducing) or $\mathrm{CM}$-arabinose (inducing) medium at $28^{\circ} \mathrm{C}$. $e I F 2 b$ was used for normalization. Expression values represent the mean of three biological replicates with two technical duplicates each. Error bars represent the SEM. (D) Immunoblot analysis of Rok1-mCherry protein levels. Derivatives of strains FB1 fuz $7^{D D}$ rokl-mCherry (WT) and FB1 fuz $7^{D D}$ rokl-mCherry cibl ${ }^{\mathrm{s}}\left(\mathrm{cib1} 1^{\mathrm{s}}\right)$ were grown in CM-glucose (non-inducing) or CM-arabinose (inducing) liquid medium for 6 hours at $28^{\circ} \mathrm{C}$. Rok1-mCherry protein was enriched from U. maydis total protein extract by immunoprecipitation with RFP-Trap beads. Total protein amount was normalized before subjected to immunoprecipitation. Rok1-mCherry was visualized using an $\alpha$-RFP [G6G] specific antibody.

\subsubsection{The UPR affects mating type-dependent signaling upstream and downstream of $\mathbf{b E} / \mathrm{bW}$}

To further examine the possibility of multiple interactions of the UPR and the pheromone and $b$-dependent signaling pathway, we tested if constitutive expression of the $b$-heterodimer is sufficient to suppress $c i b 1^{\mathrm{s}}$-dependent inhibition of filament formation and gene expression. To this end, we ectopically integrated $c i b 1^{\mathrm{s}}$ into $U$. maydis strain HA103, in which the compatible $b E 1 / b W 2$ genes are expressed under control of the constitutive active tef promoter (Hartmann et al., 1996b). Screening of two individual transformants revealed different $c i b 1^{\mathrm{s}}$ expression levels, most likely depending on the place and number of integration events (Figure 3.6A). Spotting assays on charcoal containing solid media revealed extensive filament formation of HA103 that was suppressed by expression of $\mathrm{cibl} \mathrm{I}^{\mathrm{s}}$ in a dose-dependent manner (Figure 3.6A). Consistently, expression levels of the b-target genes $r b f 1, b i z 1, h d p 1$, hdp2, dik6 and of $p r f 1$ were reduced by $c i b 1^{s}$ (Figure 3.6B). To test if the reduced filament formation and b-target gene expression in $c i b 1^{\mathrm{s}}$-expressing strains affects virulence, plant infection assays were performed. This revealed that the virulence of $c i b 1^{\mathrm{s}}$-expressing strains was significantly reduced in comparison to the HA103 WT control (Figure 3.6C). These observations are reminiscent of the results obtained with the SG200 strain in previous studies (Heimel et al., 2013) and confirm that the UPR affects regulation of the pheromone and b-dependent signaling pathways in a complex manner. 
A

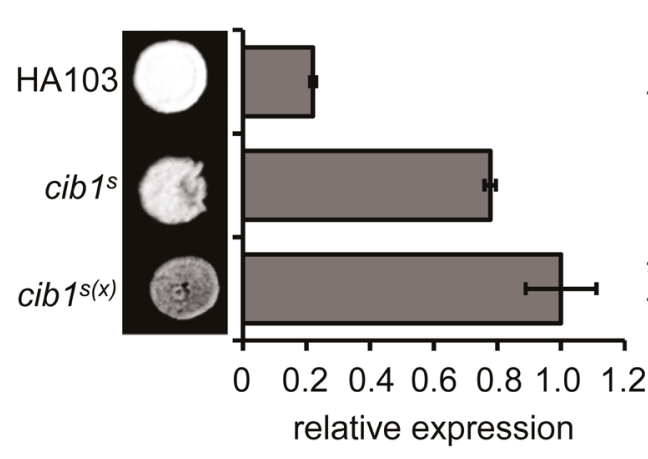

C

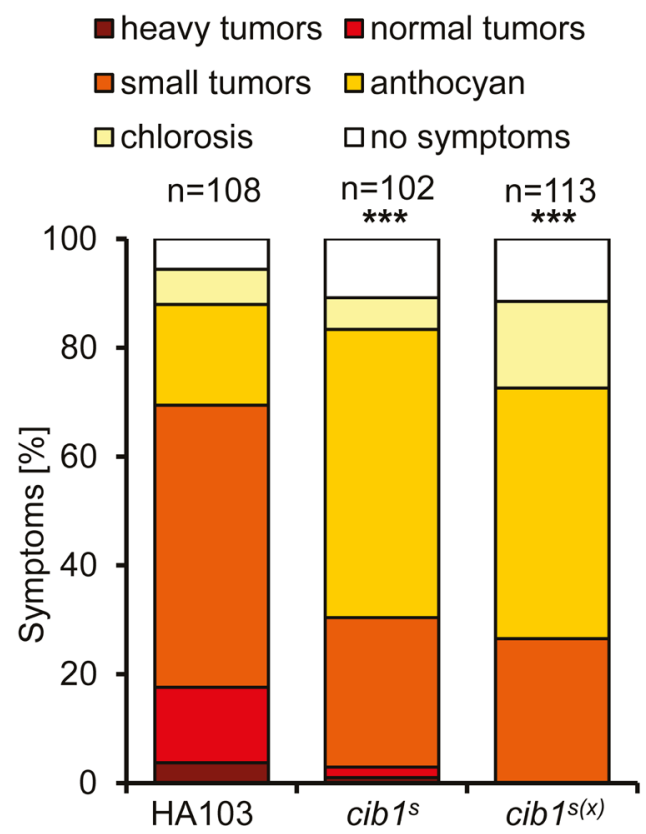

B

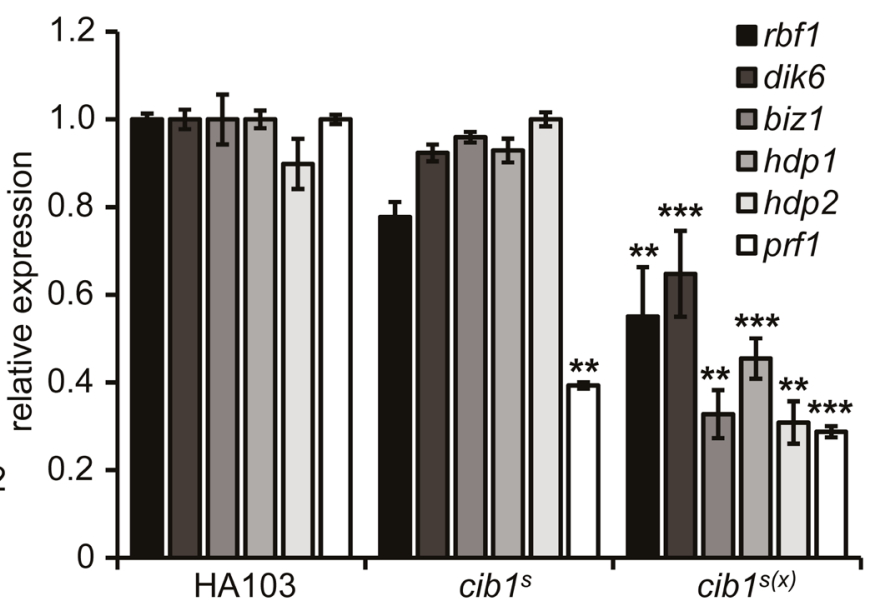

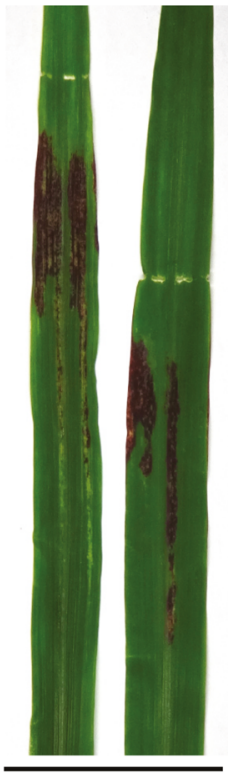

cib1s

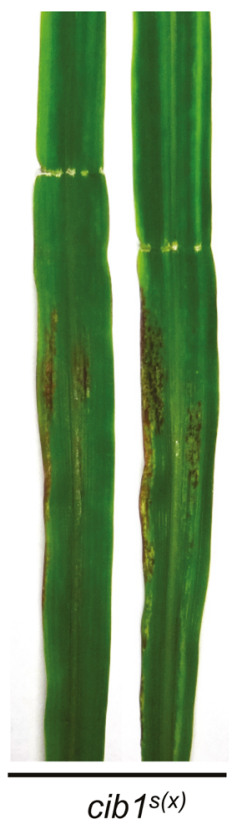

Figure 3.6 Constitutive expression of $b E / b W$ does not restore filamentous growth or gene expression. (A) Analysis of $b$-dependent filament formation. HA103 (constitutive $b E / b W$ expression) and derivatives with different $c i b 1^{\mathrm{s}}$ expression level were spotted on potato dextrose solid media supplemented with $1 \%$ charcoal. Photos were taken after 24 hours at $28^{\circ} \mathrm{C}$. White fuzzy colonies indicate the formation of filaments. (B) qRT-PCR analysis of $r b f 1$, dik6, $b i z 1, h d p 1, h d p 2$ and $p r f 1$ gene expression in strains used for the filamentous growth test in (A). eIF2b was used for normalization. Expression values represent the mean of three biological replicates with two technical duplicates each. Error bars represent the SEM. Statistical significance was calculated using the students $t$ test. $* * \mathrm{P}$ value $<0.01$, and $* * * \mathrm{P}<0.001$. (C) Plant infection assay with the solopathogenic strain HA103 and derivatives used in (A). Strains were inoculated into 8 dayold- maize seedlings. Disease symptoms were rated 8 days post inoculation (dpi) and grouped into categories as shown in the figure legend. $n=$ number of inoculated plants. Photos were taken at $8 \mathrm{dpi}$ and represent the most common infection symptom. Significance was calculated using the Mann-Whitney-test. ***P value $<$ 0.001 . 


\subsubsection{The MAPK Kpp2 is dispensable for biotrophic development in planta}

Due to the partially redundant function of Kpp2 and Kpp6, both MAPK are important but not essential for pathogenicity (Müller et al., 1999; Brachmann et al., 2003). Kpp2 is activated during the mating process and important for appressoria formation (Müller et al., 1999), whereas phosphorylation of Kpp6 is required for plant penetration (Brachmann et al., 2003). To investigate the function of Kpp2 after plant penetration, we used a conditional gene expression approach allowing the stage-specific gene expression during development in planta. FB1 $\Delta k p p 2$ and FB2 $\Delta k p p 2$ strains were transformed with constructs expressing kpp2 under control of the conditional promoters $\mathrm{P}_{U M A G_{-}} 12184$ and $\mathrm{P}_{U M A G_{-} 03597 \text {. These }}$ promoters are active during the first two $\left(\mathrm{P}_{U M A G_{-} 12184}\right)$ or four $\left(\mathrm{P}_{U M A G_{-} 03597}\right)$ days post inoculation (the detailed description of our conditional gene expression approach will be published elsewhere) but show strongly reduced activity at subsequent stages of pathogenic growth in planta (Lanver et al., 2018). We used compatible combinations of FB1 and FB2 (WT controls) and derivatives thereof for plant infection experiments. Consistent with previous studies, virulence of compatible combinations of $\Delta k p p 2$ strains was significantly impaired compared to FB1/FB2 (Figure 3.7) (Müller et al., 1999). Importantly, strains expressing $\mathrm{P}_{U M A G 12184: k p p 2}$ or $\mathrm{P}_{U M A G_{-} 03597: k p p 2}$ fully restored virulence (Figure 3.7). Hence, $\operatorname{kp} 2$ is required for the mating process and important for appressoria formation but dispensable for pathogenic development in planta. We observed that Rok1 interacts with Kpp2 and Kpp6 in the Yeast-two-Hybrid system (Suppl. Figure 3.2) and that both MAP kinases are dephosphorylated by Rok1 (Brachmann et al., 2003; Di Stasio et al., 2009). Thus, it is conceivable that the biological role of the crosstalk between UPR and the mating-type pathways might affect the function of both proteins. 
Chapter 3 | UPR-dependent inhibition of mating type signaling
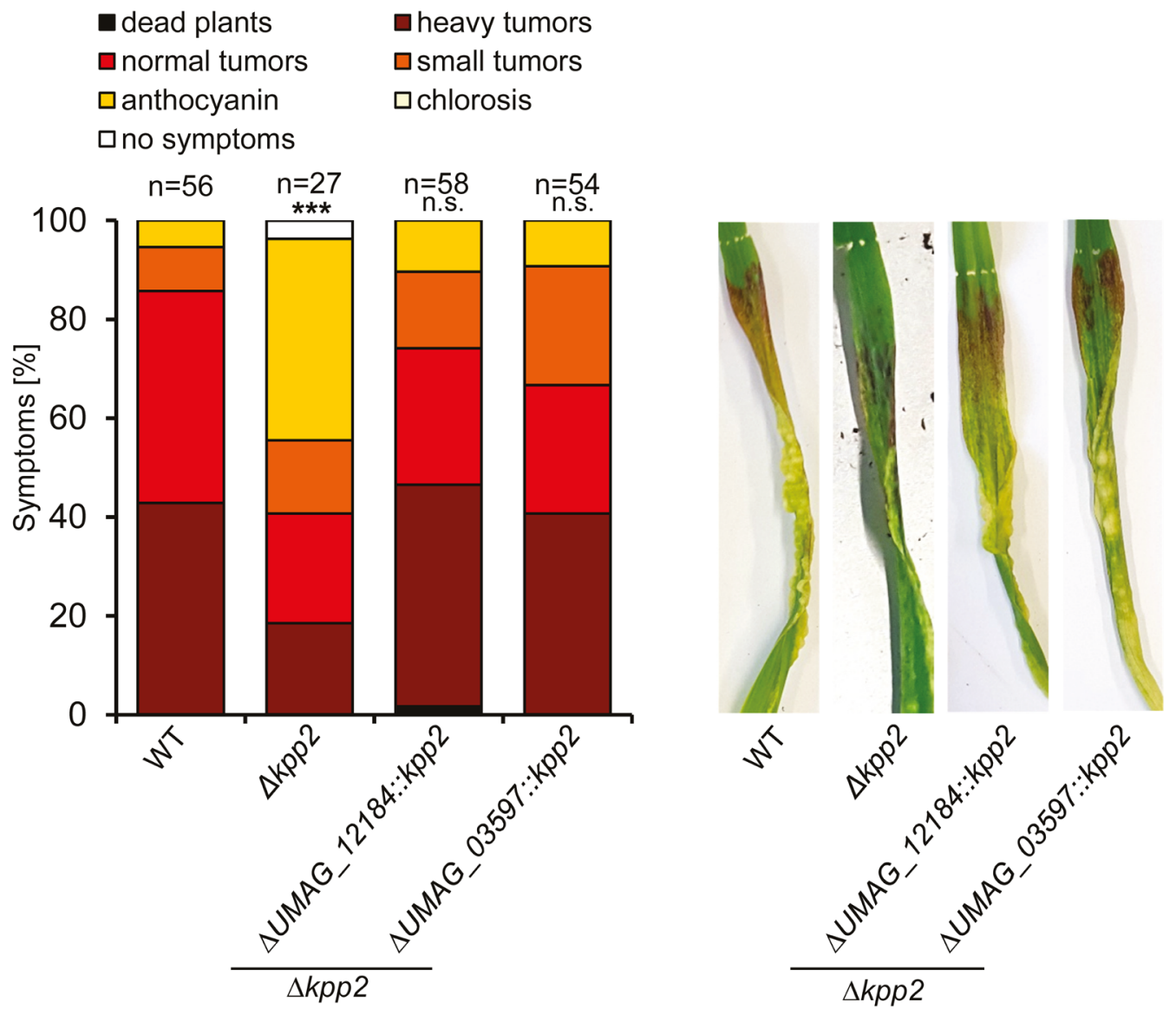

Figure 3.7 Kpp2 is dispensable for pathogenic development in planta. Plant infection assay with compatible combinations of the haploid strains FB1 x FB2 (WT), FB1 $\Delta k p p 2$ x FB2 $\Delta k p p 2(\Delta k p p 2)$ and derivatives of $\Delta k p p 2$ strains expressing $\mathrm{P}_{U M A G_{-} 12184}: k p p 2$ or $\mathrm{P}_{U M A G_{-} 03597}: k p p 2$ were inoculated into 8-day-old maize seedlings. Disease symptoms were rated 8 days after inoculation and grouped into categories as shown in the figure legend. $\mathrm{n}=$ number of inoculated plants. Photos were taken at $8 \mathrm{dpi}$ and represent the most common infection symptom. Significance was calculated using the Mann-Whitney-test. *** P value $<0.001$. 


\subsection{Discussion}

In $U$. maydis, the UPR is connected to the control of biotrophic growth via the interaction between the Hac1-like UPR regulator, Cib1, and Clp1. In this study, we show that the UPR affects pathogenic development of $U$. maydis on additional levels. While the plant-specific activation of the UPR fosters pathogenic growth in planta, premature UPR activation inhibits pathogenic development prior host plant penetration. We observed that UPR activity counteracts pheromone-dependent signaling by suppression of Kpp2 phosphorylation in a rokl-dependent manner, identifying Rok1 as a novel regulatory target of the UPR.

Expression analysis confirmed the tight connection and the extensive inhibitory effects of the UPR on the $b$-dependent transcription factor network (Heimel et al., 2013).

Analysis of the pheromone response pathway operating upstream of the b-heterodimer revealed that constitutive UPR signaling suppressed both, the morphological and transcriptional pheromone response. By contrast, in the human pathogenic fungus Cryptococcus neoformans, deletion of IRE1 resulted in increased pheromone gene expression, but defects in sexual mating (Jung et al., 2016). Sensing of compatible pheromone by haploid $U$. maydis cells results in activation of the cAMP-dependent PKA Adr1 and MAPK-mediated signaling pathway, finally leading to differential phosphorylation and activation of Prf1 by Adr1 and Kpp2. Kpp2 itself is activated by phosphorylation and regulates the transcriptional pheromone response via Prfl and the morphological response in a Prfl-independent manner (Müller et al., 2003). After pheromone treatment, both responses are inhibited by an active UPR, whereas only pheromone gene expression but not the formation of conjugation tubes was suppressed after genetic activation of the pheromone pathway. These findings are in line with previous results, illustrating that the morphological and the transcriptional response bifurcate downstream of Kpp2 (Müller et al., 2003). Importantly, phosphorylation of Kpp2 was strongly reduced by the UPR under these conditions, suggesting that Kpp2 phosphorylation is dispensable for conjugation tube formation. This contrasts the finding that strains expressing non-phosphorylatable Kpp2 $2^{\mathrm{AEF}}$ mutant proteins fail to form conjugation tubes upon pheromone stimulus (Müller et al., 2003). It thus appears possible, that the genetic activation of the pathway does not fully reflect the "natural" signaling dynamics.

The interaction between the UPR and MAPK pathways is not limited to U. maydis. In the human pathogens Aspergillus fumigatus and C. neoformans, deletion of genes encoding 
Chapter 3 | UPR-dependent inhibition of mating type signaling

homologs of Hac1 or Ire1 resulted in increased susceptibility to cell wall stress, suggesting that a functional UPR is required for and connected to the cell wall integrity (CWI) pathway (Richie et al., 2009; Cheon et al., 2011; Feng et al., 2011). By contrast, deletion of cib1 did not affect resistance to cell wall perturbing agents (Pinter et al., 2019), indicating that this function is not conserved in U. maydis. In the baker's yeast Saccharomyces cerevisiae, activation of the CWI pathway results in UPR activation (Scrimale et al., 2009) and the UPR is augmented by delayed PKA signaling, leading to an extended secondary response to ER stress (Pincus et al., 2014). However, since UPR activity in U. maydis did not affect PKA-dependent induction of the pheromone pathway genes $m f a l$ and pral, it appears unlikely that PKA signaling is connected to the inhibitory role of the UPR on the pheromone pathway in U. maydis.

Deletion of the DSP rok1 restored phosphorylation of Kpp2 in strains with active UPR, indicating a UPR-dependent regulation of Rok1. To allow tight control of signaling, MAP kinase phosphatases (MKP) are often regulated on multiple levels, including transcriptional and post-translational control (González-Rubio et al., 2019). Transcription of the rok1 ortholog in S. cerevisiae, MSG5, is induced by the pheromone stimulus placing Msg5p in a negative feedback loop with the pheromone signaling cascade (Doi et al., 1994). Similarly, rokl transcript levels in U. maydis were elevated upon activation of the MAPK module (Di Stasio et al., 2009). However, since neither transcript nor Rok1 protein levels were altered by an active UPR, additional, post-translational mechanisms regulating Rok1 activity under these conditions must exist. Post-translational modifications of MKPs like phosphorylation and ubiquitination can alter subcellular localization, stability and specificity of phosphatases. S. cerevisiae Msg5p is phosphorylated by its MAPK targets Fus3 and Slt2 as part of a regulatory feedback mechanism (Doi et al., 1994; Flández et al., 2004). In the hemibiotrophic blast fungus Magnaporthe oryzae (Pyricularia oryzae), the MKP Pmp1 dephosphorylates the MAPKs Pmk1 (Fus3/Kpp2 ortholog) and Mps1 upon phosphorylation at a conserved serine residue (Wang et al., 2017). Accordingly, two potential Rok1 phosphorylation sites have been predicted in U. maydis (Di Stasio, 2009a), but the role of phosphorylation in activation of Rok1 has not been addressed, yet.

Although deletion of rokl restored Kpp2 phosphorylation, transcription of the genes $m f a 1$, pral and prfl was still inhibited, indicating that phosphorylation of Kpp2 is not sufficient to trigger Prf1-dependent gene expression under these conditions. This points towards existence of an additional layer of UPR/MAPK crosstalk that suppresses Prf1 function 
and/or transcription. In line with this assumption, CHIPseq analysis revealed binding of Cibl to the prfl promoter. However, under the conditions tested, no influence on $\operatorname{prfl}$ gene expression was observed (Pinter et al., 2019). Alternatively, prfl might be suppressed via Hap2 and Rop1 (Brefort et al., 2005; Mendoza-Mendoza et al., 2009b), both of which are required for $p r f l$ expression and transcriptionally suppressed by the UPR. Since these three transcription factors are part of various feedback loops within the pheromone response pathway, it is speculative whether these effects are direct or indirect.

Deletion of rokl results in hypervirulence of $U$. maydis reflected by an increase in filamentous growth, appressoria formation and host colonization. These effects were connected to the elevated expression of $b E$ and $b W$ genes (Di Stasio et al., 2009). Consistently, hypervirulence was also observed in strains overexpressing the pheromone receptor Pra (FB1 P otef:pra2) (Wahl et al., 2010). This suggests that activity of the $a$ - and $b$-dependent signaling pathways is directly connected to aggressiveness of $U$. maydis. However, since $U$. maydis is a biotrophic pathogen, successful completion of its life cycle is dependent on the living host plant. Hence, effective control mechanisms are required to prevent excessive and potentially deleterious effects of host colonization. In this way, the UPR-dependently increased activity of Rok1 might quench activity of the mating type pathway to ensure the efficient and balanced development of $U$. maydis inside the host plant. Apparently, increased expression of a large number of effector-encoding genes during pathogenic development is controlled by the b-heterodimer or $b$-dependently regulated transcription factors, including Rbf1, Hdp2 and potentially Biz1 (Flor-Parra et al., 2006; Heimel et al., 2010b; Lanver et al., 2014; Lanver et al., 2018; Schmitz et al., 2018). As a consequence, increased ER stress levels would initiate a negative feedback on these regulators leading to reduced effector gene expression, allowing the fungus to recover from ER stress.

Remarkably, constitutive expression of $b E / b W$ is not sufficient to restore filamentous growth and pathogenicity in cells with active UPR. We consider it likely that i) this might result from Clp1, which becomes stabilized by the UPR and directly interferes with the function of the b-heterodimer (Heimel et al., 2010a; Heimel et al., 2013) or ii) by a cumulative effect of Clp1-dependent repression and the parallel inhibition of the central components within the pheromone pathway, some of which have been speculated to be important for pathogenic development in planta. 
Chapter 3 | UPR-dependent inhibition of mating type signaling

However, conditional gene expression suggests that $\mathrm{Kpp} 2$ is not required at later stages of biotrophic development in planta. By contrast, the MAPK Kpp6 is part of the same MAPK module, but important for appressorial function during penetration of the plant surface (Brachmann et al., 2003). Kpp6 is highly similar to Kpp2 (66\% identity) and dephosphorylated by Rok1 (Brachmann et al., 2003; Kaffarnik et al., 2003; MendozaMendoza et al., 2009a; Di Stasio et al., 2009). Thus, it is tempting to speculate that the UPR also inhibits Kpp6 function, which might be important for fungal pathogenicity after plant penetration.

Taken together, we uncovered crosstalk between the UPR and mating type-regulated pathways that affect the pathogenic program on multiple levels. We consider it likely that this is of relevance to maintain fungal biotrophy during the interaction with the host plant.

\subsection{Material and Methods}

\section{Strains and Growth Conditions}

Escherichia coli TOP10 strain was used for cloning purposes and amplification of plasmid DNA. U. maydis cells were grown at $28^{\circ} \mathrm{C}$ in YEPS light medium (Tsukuda et al., 1988), complete medium (CM) (Holliday, 1974) or yeast nitrogen base (YNB) medium (Mahlert et al., 2006; Freitag et al., 2011). Media and growth conditions used to induce gene expression driven by the $\mathrm{crgl}$ promoter was performed according to the protocol of Brachmann et al., 2001. Yeast-two-hybrid analysis was performed according to the Matchmaker 3 manual (Clontech). S. cerevisiae cells containing Y2H plasmids were grown in SD medium with -Leu/-Trp DO Supplement (Clontech) and spotted on SD solid media with -Leu/-Trp DO Supplement (Clontech) or -Ade/-His-Leu-Trp DO Supplement (Clontech). U. maydis strains used in this study are listed in Suppl. Table 3.2.

\section{DNA and RNA Procedures}

Molecular methods followed described protocols (Sambrook et al., 1989). DNA isolation from U. maydis and transformation procedures were performed according to Schulz et al., 1990. For gene deletions, a PCR-based approach and the SfiI insertion cassette system was used (Brachmann et al., 2004; Kämper, 2004). Linearized plasmid DNA or PCR amplified DNA was used for homologous integration into the genome. Correct integration was verified using Southern hybridization. Total RNA was extracted from exponentially 
growing cells in axenic culture using Trizol reagent (Invitrogen) according to the manufacturer's instructions. To check RNA integrity, total RNA was run on an agarose-gel and visualized by ethidium bromide staining. Residual DNA was removed from RNA samples using the TURBO DNA-free ${ }^{\mathrm{TM}}$ Kit (Ambion/Lifetechnologies). cDNA was synthesized using the RevertAid First Strand cDNA Synthesis Kit, Thermo Scientific. Primers used in this study are listed in Suppl. Table 3.1.

\section{Quantitative RT-PCR}

qRT-PCR analysis was performed as described before (Hampel et al., 2016). For all qRT-PCR experiments, three independent biological replicates and two technical replicates were analyzed using the MESA Green qPCR Mastermix Plus (Eurogentec). qRT-PCR was performed using the CFX Connect Real-Time PCR Detection System and analyzed with the CFX Manager Maestro Software (BioRad).

\section{Plasmid Construction}

For the $k p p 2-G F P$ fusion, $1 \mathrm{~kb}$ upstream of the kpp2 startcodon and $1 \mathrm{~kb}$ of the $k p p 2$ open reading frame (ORF) were PCR amplified from genomic DNA and ligated to the $3.7 \mathrm{~kb}$ Sfil GFP-Hyg ${ }^{R}$ fragment of pUMa317 (Becht et al., 2006). The resulting ligation product was integrated into the pCR2.1 TOPO vector (Invitrogen) generating plasmid pCR2.1 pUMa317-kpp2. For generation of the $f u z^{D D}-3 x H A$ fusion, the $f u z^{D D}$ gene was amplified from the $123 P_{\text {crgl }}: f u z 7^{D D}$ plasmid (Müller et al., 2003) adding a C-terminal HA-tag and two NdeI restriction sites. The resulting $1.3 \mathrm{~kb} N d e I f u z 7^{D D}-H A$ fragment was ligated to the

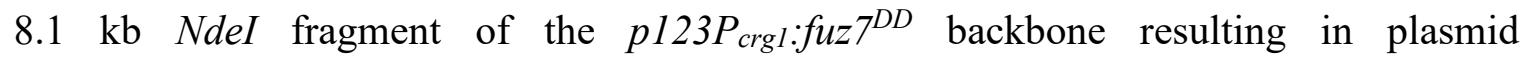
p123 fuz $7^{D D}-H A$. To generate the rokl-mCherry fusion, $1 \mathrm{~kb}$ upstream of the rokl startcodon and $1 \mathrm{~kb}$ of the rokl ORF were PCR amplified from genomic DNA and ligated to the $3.1 \mathrm{~kb}$ SfiI mCherry-G418 fragment of plasmid pUMa1723. The ligation product was integrated into the pCR2.1 TOPO vector (Invitrogen) generating plasmid pCR2.1 rokl-mCherry.

To generate rokl deletion construct, $1 \mathrm{~kb}$ upstream of the rokl start codon and $1 \mathrm{~kb}$ downstream of the rokl stop-codon were PCR amplified and ligated to the $2.4 \mathrm{~kb}$ SfiI $P_{\text {Phleo }}^{R}$ fragment of vector pUMa263. 
Chapter 3 | UPR-dependent inhibition of mating type signaling

To replace the carboxin resistance in vector $P_{c i b l}: c i b 1^{\text {s }}$ (Heimel et al., 2013) by nourseothricin resistance, the $4.2 \mathrm{~kb}$ HindIII Nat $t^{R}$ fragment of vector $\mathrm{pNEB}-\mathrm{Nat}^{\mathrm{R}}$ and the $4.1 \mathrm{~kb}$ HindIII fragment of vector $P_{c i b 1}: c i b 1^{\mathrm{s}}$ were ligated to generate the plasmid pNEB-NatR-cibl ${ }^{\text {s. }}$.

To obtain the promoter fusions/gene replacement constructs for plant infection, $1 \mathrm{~kb}$ flanking regions up- and downstream of the genes $U M A G_{-} 12184$ and $U M A G_{-} 03597$, the kpp2 ORF and the $H y g^{R}$ of vector pUMa1442 were PCR amplified. Thereby, SfiI restriction sites were added to the kpp2 gene, a SfiI restriction site was added to the 3'end of the upstream flanking region, SfiI (N-terminal) and a BamHI (3'end, for UMAG_12184) or KpnI (3'end, for $U M A G_{-}$03597) restriction site were added to the $H y g^{R}$, and a BamHI (5'end, for $U M A G_{-}$12184) or KpnI (5'end, for $U M A G \_03597$ ) restriction site was added for the downstream flanking region of the respective gene. The obtained fragments were ligated to obtain a $L B-k p p 2-H y g^{R}-R B$ fragment. The fragment was then integrated into the pCR2.1 TOPO vector (Invitrogen) generating plasmids pCR2.1 $\mathrm{P}_{U M A G_{-}}$12184:kpp2 ( $\left.\mathrm{Hyg}^{\mathrm{R}}\right)$

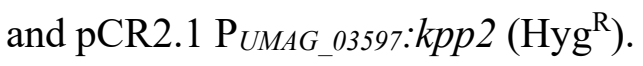

For generation of the yeast-two hybrid fusion proteins, the rok1, kpp2 and kpp6 were PCR amplified adding SfiI restriction sites and ligated to modified versions of the vectors pGBKT7 and pGADT7 (Clontech) containing SfiI restriction sites generating plasmids pGBKT7-Rok1, pGADT7-Kpp2 and pGADT7-Kpp6.

\section{Mating assay/ Filamentous growth assay}

Mating assays were performed as described in Brachmann et al., 2001. Cells were grown in $\mathrm{CM}$-glucose medium overnight, $\mathrm{OD}_{600}$ was measured and adjusted to 0.2 in fresh $\mathrm{CM}$-glucose medium. Cells were grown for $4 \mathrm{~h}$ at $28^{\circ} \mathrm{C}$. $\mathrm{OD}_{600}$ was then adjusted to $1.0 \mathrm{in}$ CM-glucose medium. For mating assay, compatible FB1 and FB2 strains and derivatives were mixed $1: 1$ and $5 \mu$ of the mixture was spotted on potato dextrose medium supplemented with 1\% charcoal (PD-CC) (Holliday, 1974). To test for filamentous growth, $5 \mu 1$ of SG200 and derivatives was spotted on PD-CC medium. Cells were grown for 2 days at $28^{\circ} \mathrm{C}$. 


\section{Pheromone and cAMP Treatment}

To test for conjugation tube formation of cells in liquid culture, cultures of $U$. maydis strains were grown for 6 hours at $28^{\circ} \mathrm{C}$ in $\mathrm{CM}$-glucose medium. $\mathrm{OD}_{600}$ was measured and adjusted in fresh CM-glucose medium to reach an $\mathrm{OD}_{600}$ of $0.25-0.4$ after 16 hours of growth at $28^{\circ} \mathrm{C}$. The following day, $2 \mu 1$ a 2 pheromone (f.c.: $2.5 \mu \mathrm{g} / \mathrm{mL}$ ) or DMSO as negative control was added to $2 \mathrm{~mL}$ of the over-night culture and cells were rotated in a $15 \mathrm{~mL}$ reaction tube on a rotating wheel with $6 \mathrm{rpm}$ for 6 hours. Cells were then microscopically analyzed using an Axio Imager.M2 equipped with an AxioCam MRm camera (ZEISS). Images were processed using ImageJ. Cells were harvested by centrifugation and flash frozen in liquid nitrogen for subsequent mRNA isolation.

For cAMP treatment, U. maydis strains were grown for $6 \mathrm{~h}$ at $28^{\circ} \mathrm{C}$ in $\mathrm{CM}$ liquid medium supplemented with $1 \%$ glucose (CM-glucose to reach an $\mathrm{OD}_{600}$ of 0.2 and treated with $6 \mathrm{mM}$ cAMP (f.c.) for $12 \mathrm{~h}$ at $28^{\circ} \mathrm{C}$, harvested by centrifugation and flash frozen in liquid nitrogen for subsequent mRNA isolation.

\section{Protein Procedures}

Protein isolation and Western hybridization experiments were performed as described in (Lanver et al., 2010). Commercially available $\alpha$-GFP (Sigma-Aldrich, 1:4000), $\alpha$-HA (Sigma-Aldrich, 1:4000) or $\alpha$-RFP [G6G] (Chromotek, 1:1000) antibodies were used to detect GFP-, HA- or mCherry-fusion proteins, respectively. Horseradish peroxidase-conjugated $\alpha$-mouse or $\alpha$-rabbit IgG (Promega, 1:4000) were used as secondary antibodies. The Luminata Crescendo Western HRP substrate (Merck Millipore) was used for chemiluminescence based detection of the fusion proteins.

For immunoprecipitation of proteins, over-night cultures of $U$. maydis were adjusted to an $\mathrm{OD}_{600}$ of 0.25 in CM supplemented with $1 \%$ glucose (non-inducing) or arabinose (induction of $\mathrm{crg} 1$-dependent gene expression) and grown for $6 \mathrm{~h}$ at $28^{\circ} \mathrm{C}$. Cells were then harvested by centrifugation, washed once with IP lysis buffer $(25 \mathrm{mM}$ Tris-HCl, pH7.4, $150 \mathrm{mM}$ $\mathrm{NaCl}, 1 \mathrm{mM}$ EDTA, 1\% NP-40, 5\% Glycerol) supplemented with 2x Complete protease inhibitor (PI, ROCHE). The pellet was frozen in liquid nitrogen and disrupted in a cell mill (Retsch MM400, 30Hz, 2 min, 2x). The disrupted cells were then resuspended in $750 \mu 1 \mathrm{IP}$ 
Chapter 3 | UPR-dependent inhibition of mating type signaling

lysis buffer $+2 \mathrm{x}$ PI and centrifuged at $22000 \mathrm{rpm}$ for $30 \mathrm{~min}$ at $4{ }^{\circ} \mathrm{C}$. The supernatant was added to $25 \mu 1$ of agarose GPF-Trap or RFP-Trap beads (Chromotek) and incubated for $3 \mathrm{~h}$ at $4^{\circ} \mathrm{C}$ on a rotating wheel. After incubation, beads were washed 3-5 times with IP lysis buffer +1 x PI. $50 \mu 1$ x Roti Load 1 (Carl-Roth) was added to the beads and boiled at $95^{\circ} \mathrm{C}$ for 5 mins. Samples were run on a 10\% (Kpp2-GFP/Fuz $7^{\mathrm{DD}}-\mathrm{HA}$ ) or $8 \%$ (Rok1-mCherry) SDS-PAGE and subsequently subjected to Western hybridization. All experiments were repeated three times.

\section{Yeast-Two-Hybrid Analysis}

Yeast-Two-Hybrid analysis was performed using the Matchmaker III system (Clontech). The S. cerevisiae strain AH109 was co-transformed with plasmids pGBKT7-Rok1 and pGADT7-Kpp2 or pGADT7-Kpp6, pGBKT7-p53 and pGADT7-T as negative control and pGBKT7-lam1 and pGADT7-T (Clontech) as positive control. Cells were grown on SD-Leu/-Trp solid media or liquid culture. To test for interaction, wells were spotted on $\mathrm{SD}-\mathrm{Ade} /-\mathrm{His} /-\mathrm{Leu} /-\mathrm{Trp}$ solid media and grown for 48 hours at $28^{\circ} \mathrm{C}$.

\section{Plant Infection Studies}

For infection studies of maize (Z. mays), the solopathogenic strain SG200 and derivatives or FB1 and FB2 and their respective derivatives were grown to an OD600 of 0.6-0.8 in YEPS light medium, adjusted to an OD600 of 1.0 in water, FB1 and FB2 strains and derivatives were mixed 1:1 with a compatible mating partner. $0.5 \mathrm{~mL}$ of the resulting suspension was used to inoculate 8-day-old maize seedlings of the variety Early Golden Bantam. Plants were grown in a CLF Plant Climatics GroBank with a $14 \mathrm{~h}\left(28^{\circ} \mathrm{C}\right)$ day and $10 \mathrm{~h}\left(22^{\circ} \mathrm{C}\right)$ night cycle. Symptoms were scored according to disease rating criteria reported by Kämper et al., 2006. Three independent clones were used for the plant infection experiment and the average scores for each symptom are shown. Photos from infected leaves were taken und represent the most common infection symptom for the respective mutant. 


\section{Statistical Analysis}

Statistical significance was calculated using students $t$ test. The statistical significance of plant infection phenotypes was calculated using the Mann-Whitney test as described previously (Freitag et al., 2011). Results were considered significant if the P value was $<0.05$.

\section{Accession numbers}

Sequence data from this article can be found in the National Center for Biotechnology Information database under the following accession numbers:

biz1, UMAG_02549, XP_011388956.1; hdp1, UMAG 12024,XP_011391576.1; hdp2, UMAG_04928,XP_011391247.1; hap2, UMAG_01597, XP_011387600.1; rop1, $U M A G \_12033, \quad$ XP_011392517.1; mfal, UMAG_02382, XP_011388682.1; pral, $U M A G \_02383, X P \_011388683.1 ; p r f 1, U M A G \_02713$, XP_011389082.1; UMAG_00306, XP_011386216.1; UMAG_05348, XP_011392050.1; UMAG_06190, XP_011392560.1; $U M A G \_10838, \quad$ XP_011389822.1; fuz7, UMAG_01514, XP_011387552.1; kpp2, UMAG_03305, XP_011389711.1; rok1, UMAG_03701, XP_011390174.1; kpp6, $U M A G \_02331, X P \_011388645.1 ; e I F 2 b, U M A G \_04869$, XP_011391708.1

\section{Supporting Information}

S1

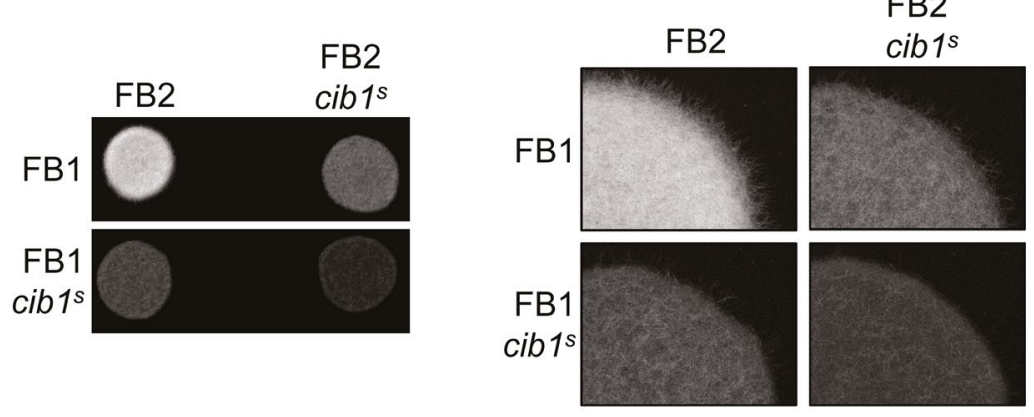

Suppl. Figure 3.1 Mating is reduced in strains expressing $c i b 1^{\mathrm{s}}$. Mating assay with of FB1, FB2, FB1 $c i b 1^{\mathrm{s}}$ and FB2 $c i b 1^{\text {s. }}$. Compatible mixtures of strains were spotted on PC-DD solid media as shown in the figure. Photos were taken after 24 hours at $28^{\circ} \mathrm{C}$. White fuzzy colonies indicate the formation of filaments. 
Chapter 3 | UPR-dependent inhibition of mating type signaling

S2

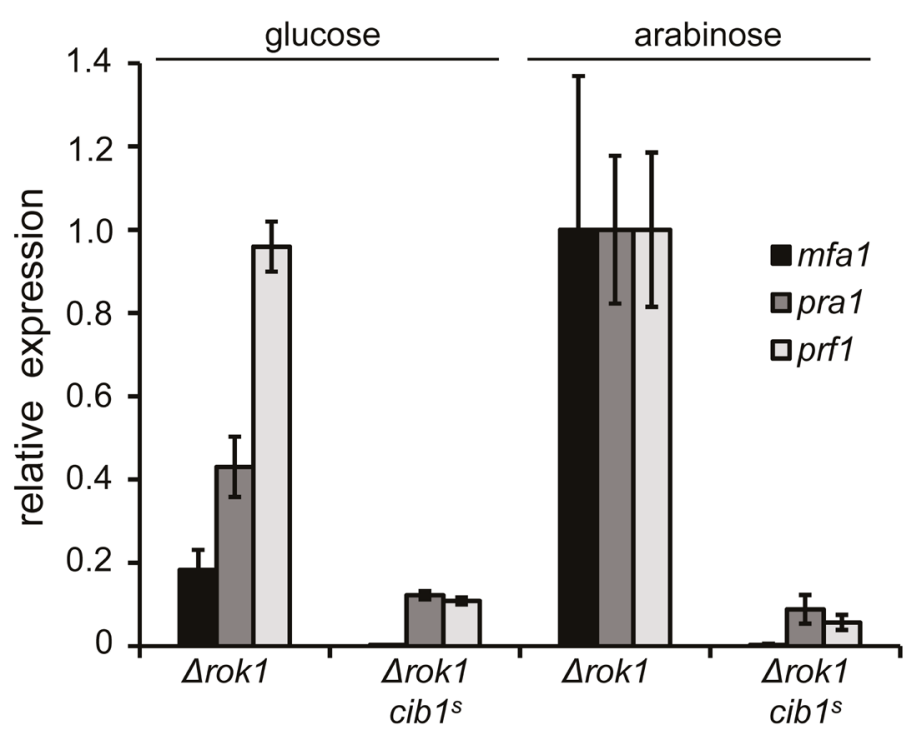

Suppl. Figure 3.2 rok1 deletion does not restore expression of the a-mating type genes and prf1. qRT-PCR analysis of $m f a 1$, pral and prfl gene expression. Derivatives of strains FB1 fuz $7^{D D}$ (WT) and FB1 $f u z 7^{D D} c i b 1^{\mathrm{s}}\left(c i b 1^{\mathrm{s}}\right)$ deleted for rokl were grown in CM-glucose (non-inducing) or CM-arabinose (inducing) liquid medium for 6 hours at $28^{\circ} \mathrm{C}$. eIF $2 b$ was used for normalization. Expression values represent the mean of three biological replicates with two technical duplicates each. Error bars represent the SEM.

S3

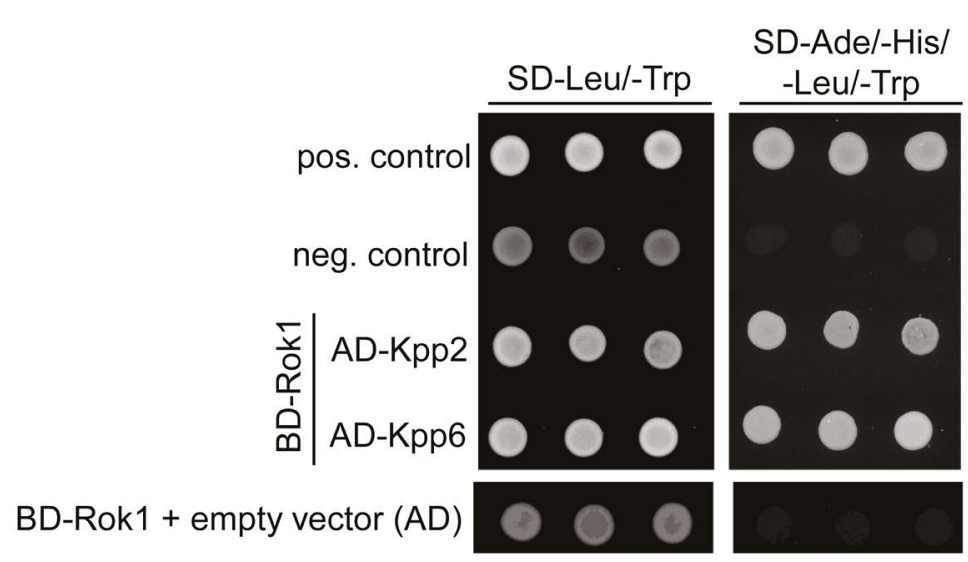

Suppl. Figure 3.3 Rok1 does physically interact with the MAPKs Kpp2 and Kpp6. Yeast strain AH109 was transformed with pGBKT7-Rok1 and pGADT7-Kpp2 or pGBKT7-Rok1 and pGADT7-Kpp6 and spotted on SD-Leu/-Trp and SD-Ade/-His/-Leu/-Trp solid media. AH109 transformed with pGBKT7-p53 and pGADT7-T or pGBKT7-lam1 and pGADT7-T (Clontech) served as positive and negative control, respectively. Yeast strain AH109 was transformed with pGBKT7-Rok1 and pGADT7 and spotted on SD-Leu/-Trp and SD-Ade/-His/-Leu/-Trp solid media to test for auto-activation of Rok1. 
Suppl. Table 3.1 Primers used in this study

\begin{tabular}{|c|c|c|c|}
\hline Purpose & Name & Sequence & Reference \\
\hline \multirow[t]{32}{*}{ qRT-PCR } & RT_eIF2b_f & ATCCCGAACAGCCCAAAC & Heimel et al., 2010a \\
\hline & RT_eIF2b_r & ATCGTCAACCGCAACCAC & Heimel et al., 2010a \\
\hline & RT-Biz1_f & CTGACCGAGGCTACTGGC & This study \\
\hline & RT-Biz1_r & ATCAATCGGCGGGTTGGATG & This study \\
\hline & RT_hdp1_f & CCGAAAGCGTCTGGGATGAG & Kellner et al., 2014 \\
\hline & RT_hdp1_r & GTCGTGCGTACATCGTACGG & Kellner et al., 2014 \\
\hline & RT_hdp2_f & GGCGCTTTGCATTGGAAC & Kellner et al., 2014 \\
\hline & RT_hdp2_r & AGCTTGAAGCCGATCGAC & Kellner et al., 2014 \\
\hline & RT_hap2-f & AGACTCGCGACAAGGCCC & This study \\
\hline & RT_hap2_r & CCTCCAGGACCTCTTGGC & This study \\
\hline & RT_rop1_f & ACTGGCTACACCACAATCGCC & This study \\
\hline & RT_rop1_r & TGACGCGGTGGCTGCTGTT & This study \\
\hline & RT_mfa1_f & ATGCTTTCGATCTTCGCTCAGAC & Heimel et al., 2010 \\
\hline & RT_mfal_r & TAGCCGATGGGAGAACCGTTG & Heimel et al., 2010 \\
\hline & RT_pral_f & AACCGAAGGCATCTGCACTGC & Heimel et al., 2010 \\
\hline & RT_pra1_r & CCCGCATGTCGATGTCAGACT & Heimel et al., 2010 \\
\hline & RT_prf1_f & TCGGTAGAACGAGCTGTGATG & Zarnack et al., 2008 \\
\hline & RT_prf1_r & CTGTTGGACGATGTTGGAGTTG & Zarnack et al., 2008 \\
\hline & RT-um05348-f & CGAGGGACGAAATCGTGGTC & This study \\
\hline & RT-um05348-r & CAGTCCATGAACGCTTTTGCTC & This study \\
\hline & RT-um10838-f & GGCTCAAGACGATCTCGTTCA & This study \\
\hline & RT-um10838-r & CCCGTTCCATCGATTCTGCAT & This study \\
\hline & RT-um06190-f & AGTATTGGGACGACTGCTCCG & This study \\
\hline & RT-um06190-r & ATGCTGATACCGGCGATGCC & This study \\
\hline & RT-um00306-f & GTGAGTGGATGGCGTTTAGCA & This study \\
\hline & RT-um00306-r & TTCGCTTCGTGAATGTACCATG & This study \\
\hline & $\mathrm{RT}$ rok1_r & GAGCAGGATATCCACCTTGC & This study \\
\hline & RT_rok1_f & CTGTCTTCTTCGTCGTCGTC & This study \\
\hline & RT_rbf1_f & AGTACGAGCTACGACGGATTC & Scherer et al., 2006 \\
\hline & RT_rbf1_r & GGGTAGGTGTTGGACACATTC & Scherer et al., 2006 \\
\hline & RT_dik6_f & TTGTTCCACCCATCCTTCACGC & Scherer et al., 2006 \\
\hline & RT_dik6_r & GGATCGAGCGTCGAAACACAGC & Scherer et al., 2006 \\
\hline \multirow[t]{5}{*}{ Kpp2-GFP } & kpp2_fus_lf & CGTACAAGGTCGTCGATGTG & This study \\
\hline & kpp2_fus_lr & TTTGGCCGCGTTGGCCGCACGCAT & This study \\
\hline & & GATCTCGTTATAAATCAAC & \\
\hline & kpp2_fus_rf & AAAGGCCTGAGTGGCCCGAATTCG & \\
\hline & & CTCCGCTCTAG & \\
\hline
\end{tabular}


Chapter 3 | UPR-dependent inhibition of mating type signaling

\begin{tabular}{|c|c|c|c|}
\hline Purpose & Name & Sequence & Reference \\
\hline & kpp2_fus_rr & GCACTGGCCTCAACTCTAAG & \\
\hline \multirow{5}{*}{$\begin{array}{l}\mathrm{Fuz} 7^{\mathrm{DD}}- \\
\mathrm{HA}\end{array}$} & Fuz7DD-HA_f & TATACATATGCTTTCGTCCGGTGC & This study \\
\hline & & & \\
\hline & Fuz7DD-HA_r & TATACATATGTTAGGCATAGTCGG & This study \\
\hline & & GCACGTCGTAGGGGTACTTCATCC & \\
\hline & & CATCGGCCCAT & \\
\hline \multirow{5}{*}{$\begin{array}{l}\text { Rok1- } \\
\text { mCherry }\end{array}$} & rok1_fus_rf & TTTGGCCTGAGTGGCCACTTGAAT & This study \\
\hline & & CAATATTTTTGAGGATGCG & \\
\hline & rok1_fus_lf & GCTCAGGATCGACTCGAGG & This study \\
\hline & rok1_fus_lr & TTTGGCCGCGTTGGCCGCCGAGAG & This study \\
\hline & & GCTGTCGCTGTC & \\
\hline \multirow{6}{*}{$\begin{array}{l}\text { rokl } \\
\text { deletion }\end{array}$} & rok1_KO_lf & CAGCTGGCACTTTGTCACTCACG & This study \\
\hline & rok1_KO_lr & GTGGGCCATCTAGGCCGTGTGTGG & This study \\
\hline & & TCGAGATTTGACAGC & \\
\hline & rok1_KO_rf & CACGGCCTGAGTGGCCGGATGCGA & This study \\
\hline & & TCAACACATACAGTGAAGG & \\
\hline & rok1_KO_rr & GTTGCCAACTCTGTCGGCTATCG & This study \\
\hline \multirow{16}{*}{$\begin{array}{l}\text { kpp2 } \\
\text { promoter } \\
\text { fusion }\end{array}$} & kpp2_SfiI_for & TATAGGCCTGAGTGGCCATGTCAC & This study \\
\hline & & ATGCCCACGGAC & \\
\hline & kpp2_SfiI_rev & TATAGGCCATCTAGGCCTCAACGC & This study \\
\hline & & ATGATCTCGTTATAAATC & \\
\hline & Hyg_f & TATAGGCCTAGATGGCCAGAAGTT & This study \\
\hline & & $\mathrm{C}$ & \\
\hline & Hyg_r_BamHI & TATAGGATCCGAAGTTCCTATACT & This study \\
\hline & & TTCTAGAGAATAG & \\
\hline & um12184_LB_f & CCAAGGCTAACGTGATGGATAC & This study \\
\hline & um12184_lr_SfiI & TATAGGCCACTCAGGCCGGTAACT & This study \\
\hline & & TTCTGTCTTGTACAAGGT & \\
\hline & um12184_rf_BamHI & TATAGGATCCAGCAGAGTAGGAA & This study \\
\hline & & CGAGTGGT & \\
\hline & um12184_RB_r & GACTCACAGACTCGACTGTG & This study \\
\hline & um03597_KO_lf & GGTGCCAGAGAGGATGCAAG & This study \\
\hline & um03597_KO_rr & GACGGTAACAACGCGTATACC & This study \\
\hline
\end{tabular}




\begin{tabular}{|c|c|c|c|}
\hline Purpose & Name & Sequence & Reference \\
\hline & um03597_lr_SfiI & TATAGGCCACTCAGGCCGGTGCAG & This study \\
\hline & & AGCTGACGGAAG & \\
\hline & um03597_rf_KpnI & TATAGGTACCCGTCAAGTCAACTT & This study \\
\hline & & TACCGGTC & \\
\hline \multirow[t]{12}{*}{$\mathrm{Y} 2 \mathrm{H}$} & kpp2_Y2H_for & TATAGGCCATTACGGCCATGTCAC & This study \\
\hline & & ATGCCCACGGAC & \\
\hline & Y2H_kpp2_rev & ATATGGCCGAGGCGGCCTCAACGC & This study \\
\hline & & ATGATCTCGTTATAAATC & \\
\hline & rok1_Y2H_for & TATAGGCCATTACGGCCATGGATT & This study \\
\hline & & TACGCAACGCTAACCT & \\
\hline & $\mathrm{Y} 2 \mathrm{H} \_r o k 1 \_r e v$ & ATATGGCCGAGGCGGCCCTACGAG & This study \\
\hline & & AGGCTGTCGCT & \\
\hline & kpp6_Y2H_for & TATAGGCCATTACGGCCATGTCGA & This study \\
\hline & & TTGCCAATGCCTCTT & \\
\hline & Y2H_kpp6_rev & ATATGGCCGAGGCGGCCTCAACGA & This study \\
\hline & & AGAAGCGGCTGAAAT & \\
\hline
\end{tabular}

Suppl. Table 3.2 Strains used in this study

\begin{tabular}{|c|c|c|}
\hline Strain & Relevant Genotype & Reference \\
\hline \multirow[t]{2}{*}{ FB1 } & $a l b 1$ & Banuett and \\
\hline & & Herskowitz, 1989 \\
\hline \multirow[t]{2}{*}{ FB2 } & $a 2 b 2$ & Banuett and \\
\hline & & Herskowitz, 1989 \\
\hline HA103 & $a 1 b W 2 b E 1^{c o n}$ & Hartmann et al., 1996 \\
\hline SG200 & $a 1 m f a 2 b E 1 b W 2$ & Kämper et al., 2006 \\
\hline SG200 cibl $^{\text {s }}$ & almfa2 bE1bW2 ip $p^{r} \mathrm{P}_{c i b l}: c i b 1^{s} i p^{s}$ & Heimel et al., 2013 \\
\hline SG200 $\operatorname{cib1} 1^{\mathrm{s}(\mathrm{x})}$ & almfa2 bE1bW2 mip ${ }^{r} \mathrm{P}_{c i b l}: c i b 1^{s} i p^{s}$ & Heimel et al., 2013 \\
\hline FB1 $\operatorname{cib1} 1^{\text {s }}$ (ULS1) & al b1 ip $p^{r} \mathrm{P}_{c i b l}: c i b 1^{s} i p^{s}$ & This study \\
\hline FB1 $\operatorname{cib1} 1^{\mathrm{s}(\mathrm{x})}(\mathrm{ULS} 2)$ & al bl mip ${ }^{r} \mathrm{P}_{c i b l}: c i b 1^{s} i p^{s}$ & This study \\
\hline FB2 $\operatorname{cib1} 1^{\mathrm{s}(\mathrm{x})}(\mathrm{ULS3})$ & a2 b2 mip ${ }^{r} \mathrm{P}_{c i b l}: c i b 1^{s} i p^{s}$ & This study \\
\hline HA103 $\operatorname{cibl}^{\mathrm{s}}$ (ULS5) & $a 1 b W 2 b E 1^{c o n} \mathrm{P}_{c i b 1}: c i b 1^{s}$ & This study \\
\hline HA103 $\operatorname{cib1} 1^{\mathrm{s}}$ (ULS7) & $a 1 b W 2 b E 1^{c o n} \mathrm{P}_{c i b l}: c i b 1^{s}$ & This study \\
\hline FB1 $f u z 7^{D D}($ ULS29) & al b1 ip ${ }^{r} \mathrm{P}_{c r g}: f u z 7^{D D} i p^{s}$ & This study \\
\hline FB1 $f u z 7^{D D} c i b 1^{\mathrm{s}}$ & al b1 ip ${ }^{r} \mathrm{P}_{c r g}: f u z 7^{D D} i p^{s} \mathrm{P}_{c i b l}: c i b 1^{s}$ & This study \\
\hline \multicolumn{3}{|l|}{ (ULS35) } \\
\hline ULS61 & al b1 ip ${ }^{r} \mathrm{P}_{c r g}: f u z 7^{D D} i p^{s} \mathrm{P}_{k p p 2}: k p p 2-G F P$ & This study \\
\hline ULS111 & al b1 ip $p^{r} \mathrm{P}_{c r g}: f u z 7^{D D} i p^{s} \mathrm{P}_{k p p 2}: k p p 2-G F P \mathrm{P}_{c i b 1}: c i b 1^{s}$ & This study \\
\hline
\end{tabular}


Chapter 3 | UPR-dependent inhibition of mating type signaling

\begin{tabular}{|c|c|c|}
\hline Strain & Relevant Genotype & Reference \\
\hline ULS139 & al b1 ip ${ }^{r} \mathrm{P}_{c r g}: f u z 7^{D D} i p^{s} \mathrm{P}_{k p p 2}: k p p 2-G F P \Delta r o k 1$ & This study \\
\hline ULS142 & $\begin{array}{l}\text { al b1 ip }{ }^{r} \mathrm{P}_{c r g}: f u z 7^{D D} i p^{s} \mathrm{P}_{k p p 2}: k p p 2-G F P \mathrm{P}_{c i b 1}: c i b 1^{s} \\
\text { srok1 }\end{array}$ & This study \\
\hline ULS147 & al b1 $\mathrm{P}_{k p p 2: k p p 2-G F P} i p^{r} \mathrm{P}_{c r g}: f u z 7^{D D}-3 x H A i p^{s}$ & This study \\
\hline ULS161 & $\begin{array}{l}\text { al b1 } \mathrm{P}_{k p p 2}: k p p 2-G F P \mathrm{P}_{c i b 1}: c i b 1^{s} i p^{r} \mathrm{P}_{c r g}: f u z 7^{D D_{-}} \\
3 x H A i p^{s}\end{array}$ & This study \\
\hline ULS162 & $\begin{array}{l}\text { al b1 ip }{ }^{r} \mathrm{P}_{c r g}: f u z 7^{D D} i p^{s} \mathrm{P}_{k p p 2}: k p p 2-G F P \mathrm{P}_{r o k 1}: \text { rok1- } \\
\text { mCherry }\end{array}$ & This study \\
\hline ULS165 & $\begin{array}{l}\text { al b1 ip }{ }^{r} \mathrm{P}_{c r g}: f u z 7^{D D} i p^{s} \mathrm{P}_{k p p 2}: k p p 2-G F P \mathrm{P}_{c i b 1}: c i b 1^{s} \\
\mathrm{P}_{\text {rokl }}: \text { rok1-mCherry }\end{array}$ & This study \\
\hline ULS169 & al b1 $\mathrm{P}_{c r g}: f u z 7^{D D}-3 x H A$ ip ${ }^{s} \mathrm{P}_{\text {otef }}: G F P$ & This study \\
\hline ULS302 & $a 2 b 2 \Delta k p p 2 \triangle U M A G_{-} 12184: \because k p p 2$ & This study \\
\hline ULS331 & al b1 $\Delta$ kpp2 $\triangle U M A G_{-} 12184: \because k p p 2$ & This study \\
\hline ULS343 & $a 2$ b2 $\Delta$ kpp2 $\triangle U M A G \_03597: \because k p p 2$ & This study \\
\hline ULS355 & al b1 $\Delta$ kpp2 $\triangle U M A G \_03597: \because k p p 2$ & This study \\
\hline
\end{tabular}

\section{Acknowledgements}

This work was supported by funding from the Deutsche Forschungsgemeinschaft (DFG) in the framework of the IRTG 2172 PRoTECT. We thank Regine Kahmann for strains, plasmids and the generous gift of synthetic a2 pheromone and Gerhard Braus and Ivo Feussner for generous support.

\section{References}

Andrews, D.L., Egan, J.D., Mayorga, M.E., and Gold, S.E. (2000) The Ustilago maydis $u b c 4$ and $u b c 5$ Genes Encode Members of a MAP Kinase Cascade Required for Filamentous Growth. Mol Plant-Microbe Interact 13: 781-786.

Banuett, F. (1992) Ustilago maydis, the delightful blight. Trends Genet 8: 174-180.

Banuett, F., and Herskowitz, I. (1994) Identification of Fuz7, a Ustilago maydis MEK/MAPKK homolog required steps in the fungal life cycle. Genes Dev 8: 1367-1378.

Becht, P., König, J., and Feldbrügge, M. (2006) The RNA-binding protein Rrm4 is essential for polarity in Ustilago maydis and shuttles along microtubules. J Cell Sci 119: 4964-4973. 
Bölker, M., Genin, S., Lehmler, C., and Kahmann, R. (1995) Genetic regulation of mating and dimorphism in Ustilago maydis. Can J Bot 73: 320-325.

Bölker, M., Urban, M., and Kahmann, R. (1992) The a mating type locus of U. maydis specifies cell signaling components. Cell 68: 441-450.

Brachmann, A., König, J., Julius, C., and Feldbrügge, M. (2004) A reverse genetic approach for generating gene replacement mutants in Ustilago maydis. Mol Genet Genomics 272: 216-226.

Brachmann, A., Schirawski, J., Müller, P., and Kahmann, R. (2003) An unusual MAP kinase is required for efficient penetration of the plant surface by Ustilago maydis. EMBO J 22: 2199-2210.

Brachmann, A., Weinzierl, G., Kämper, J., and Kahmann, R. (2001) Identification of genes in the bW/bE regulatory cascade in Ustilago maydis. Mol Microbiol 42: 1047-1063.

Brefort, T., Müller, P., and Kahmann, R. (2005) The High-Mobility-Group Domain Transcription Factor Rop1 Is a Direct Regulator of prf1 in Ustilago maydis. Eukaryot Cell 4: 379-391.

Cheon, S.A., Jung, K.W., Chen, Y.L., Heitman, J., Bahn, Y.S., and Kang, H.A. (2011) Unique evolution of the UPR pathway with a novel bZIP transcription factor, HxL1, for controlling pathogenicity of Cryptococcus neoformans. PLoS Pathog 7: 1-16.

Choi, K.-Y., Satterberg, B., Lyons, D.M., and Elion, E.A. (1994) Ste5 Tethers Multiple Protein Kinases in the MAP Kinase Cascade Required for Mating in S. cerevisiae. Cell 78: $499-512$.

Cox, J.S., Chapman, R.E., and Walter, P. (1997) The unfolded protein response coordinates the production of endoplasmic reticulum protein and endoplasmic reticulum membrane. Mol Biol Cell 8: 1805-1814.

Dard, N., and Peter, M. (2006) Scaffold proteins in MAP kinase signaling: more than simple passive activating platforms. BioEssays 28: 146-156.

Doi, K., Gartner, A., Ammerer, G., Errede, B., Shinkawa, H., Sugimoto, K., and Matsumoto, K. (1994) MSG5, a novel protein phosphatase promotes adaptation to pheromone response in S. cerevisiae. EMBO J 13: 61-70. 
Chapter 3 | UPR-dependent inhibition of mating type signaling

Feng, X., Krishnan, K., Richie, D.L., Aimanianda, V., Hartl, L., Grahl, N., et al. (2011) HacA-Independent Functions of the ER Stress Sensor IreA Synergize with the Canonical UPR to Influence Virulence Traits in Aspergillus fumigatus. PLoS Pathog 7: 1-18.

Flández, M., Cosano, I.C., Nombela, C., Martín, H., and Molina, M. (2004) Reciprocal Regulation between Slt2 MAPK and Isoforms of Msg5 Dual-specificity Protein Phosphatase Modulates the Yeast Cell Integrity Pathway. J Biol Chem 279: 11027-11034. Flor-Parra, I., Vranes, M., Kämper, J., and Perez-Martin, J. (2006) Biz1, a Zinc Finger Protein Required for Plant Invasion by Ustilago maydis, Regulates the Levels of a Mitotic Cyclin. Plant Cell Online 18: 2369-2387.

Freitag, J., Lanver, D., Böhmer, C., Schink, K.O., Bölker, M., and Sandrock, B. (2011) Septation of infectious hyphae is critical for appressoria formation and virulence in the smut fungus Ustilago maydis. PLoS Pathog 7: 1-15.

Gillissen, B., Bergemann, J., Sandmann, C., Schroeer, B., Bölker, M., and Kahmann, R. (1992) A two-component regulatory system for self/non-self recognition in Ustilago maydis. Cell 68: 647-657.

González-Rubio, G., Fernández-Acero, T., Martín, H.H., and Molina, M. (2019) MitogenActivated Protein Kinase Phosphatases (MKPs) in Fungal Signaling: Conservation, Function, and Regulation. Int J Mol Sci 20: 1-16.

Hampel, M., Jakobi, M., Schmitz, L., Meyer, U., Finkernagel, F., Doehlemann, G., and Heimel, K. (2016) Unfolded Protein Response (UPR) Regulator Cib1 Controls Expression of Genes Encoding Secreted Virulence Factors in Ustilago maydis. PLoS One 11: 1-16.

Hartmann, H.A., Kahmann, R., and Bölker, M. (1996) The pheromone response factor coordinates filamentous growth and pathogenicity in Ustilago maydis. EMBOJ 15: 16321641

Heimel, K., Freitag, J., Hampel, M., Ast, J., Bolker, M., and Kamper, J. (2013) Crosstalk between the Unfolded Protein Response and Pathways That Regulate Pathogenic Development in Ustilago maydis. Plant Cell 25: 4262-4277.

Heimel, K., Scherer, M., Schuler, D., Kamper, J., Kämper, J., Heimel, K., et al. (2010a) The Ustilago maydis Clp1 Protein Orchestrates Pheromone and $b$-Dependent Signaling 
Pathways to Coordinate the Cell Cycle and Pathogenic Development. Plant Cell 22: 29082922.

Heimel, K., Scherer, M., Vranes, M., Wahl, R., Pothiratana, C., Schuler, D., et al. (2010b)

The transcription factor Rbfl is the master regulator for $b$-mating type controlled pathogenic development in Ustilago maydis. PLoS Pathog 6: 1-13.

Hetz, C. (2012) The unfolded protein response: Controlling cell fate decisions under ER stress and beyond. Nat Rev Mol Cell Biol 13: 89-102.

Holliday, R. (1974) GENETICS Volume 1 Bacteria, Bacteriophages, and Fungi.

Jung, K.-W., So, Y.-S., and Bahn, Y.-S. (2016) Unique roles of the unfolded protein response pathway in fungal development and differentiation. Sci Rep 6: 1-14.

Kaffarnik, F., Müller, P., Leibundgut, M., Kahmann, R., and Feldbrügge, M. (2003) PKA and MAPK phosphorylation of Prf1 allows promoter discrimination in Ustilago maydis. EMBO J 22: 5817-5826.

Kämper, J. (2004) A PCR-based system for highly efficient generation of gene replacement mutants in Ustilago maydis. Mol Genet Genomics 271: 103-110.

Kämper, J., Kahmann, R., Bölker, M., Ma, L.-J., Brefort, T., Saville, B.J., et al. (2006) Insights from the genome of the biotrophic fungal plant pathogen Ustilago maydis. Nature 444: $97-101$.

Kämper, J., Reichmann, M., Romeis, T., Bölker, M., and Kahmann, R. (1995) Multiallelic recognition: Nonself-dependent dimerization of the $\mathrm{bE}$ and $\mathrm{bW}$ homeodomain proteins in Ustilago maydis. Cell 81: 73-83.

Kawahara, T., Yanagi, H., Yura, T., and Mori, K. (1998) Unconventional Splicing of HAC1/ERN4 mRNA Required for the Unfolded Protein Reponse. Biochemistry 273: 18021807.

Lanver, D., Mendoza-Mendoza, A., Brachmann, A., and Kahmann, R. (2010) Sho1 and Msb2-Related Proteins Regulate Appressorium Development in the Smut Fungus Ustilago maydis. Plant Cell 22: 2085-2101.

Lanver, D., Berndt, P., Tollot, M., Naik, V., Vranes, M., Warmann, T., et al. (2014) Plant Surface Cues Prime Ustilago maydis for Biotrophic Development. PLoS Pathog 10: 1-14. 
Chapter 3 | UPR-dependent inhibition of mating type signaling

Lanver, D., Müller, A.N., Happel, P., Schweizer, G., Haas, F.B., Franitza, M., et al. (2018) The Biotrophic Development of Ustilago maydis Studied by RNA-Seq Analysis. Plant Cell 30: $300-323$.

Lanver, D., Tollot, M., Schweizer, G., Presti, L. Lo, Reissmann, S., Ma, L.S., et al. (2017) Ustilago maydis effectors and their impact on virulence. Nat Rev Microbiol 15: 409-421.

Mahlert, M., Leveleki, L., Hlubek, A., Sandrock, B., and Bölker, M. (2006) Rac1 and $\mathrm{Cdc} 42$ regulate hyphal growth and cytokinesis in the dimorphic fungus Ustilago maydis. Mol Microbiol 59: 567-578.

Mayorga, M.E., and Gold, S.E. (1999) A MAP kinase encoded by the $u b c 3$ gene of Ustilago maydis is required for filamentous growth and full virulence. Mol Microbiol 34: 485-497.

Mendoza-Mendoza, A., Berndt, P., Djamei, A., Weise, C., Linne, U., Marahiel, M., et al. (2009a) Physical-chemical plant-derived signals induce differentiation in Ustilago maydis. Mol Microbiol 71: 895-911.

Mendoza-Mendoza, A., Eskova, A., Weise, C., Czajkowski, R., and Kahmann, R. (2009b) Hap2 regulates the pheromone response transcription factor prf1 in Ustilago maydis. Mol Microbiol 72: 683-698.

Müller, P., Aichinger, C., Feldbrügge, M., and Kahmann, R. (1999) The MAP kinase Kpp2 regulates mating and pathogenic development in Ustilago maydis. Mol Microbiol 34: 1007-1017.

Müller, P., Weinzierl, G., Brachmann, A., Feldbrügge, M., and Kahmann, R. (2003) Mating and Pathogenic Development of the Smut Fungus Ustilago maydis Are Regulated by One Mitogen-Activated Protein Kinase Cascade. Eukaryot Cell 2: 1187-1199.

Pincus, D., Aranda-Diaz, A., Zuleta, I.A., Walter, P., and El-Samad, H. (2014) Delayed Ras/PKA signaling augments the unfolded protein response. Proc Natl Acad Sci 111: $14800-14805$.

Pinter, N., Hach, C.A., Hampel, M., Rekhter, D., Zienkiewicz, K., Feussner, I., et al. (2019) Signal peptide peptidase activity connects the unfolded protein response to plant defense suppression by Ustilago maydis. PLOS Pathog 15: 1-40.

Presti, L. Lo, Lanver, D., Schweizer, G., Tanaka, S., Liang, L., Tollot, M., et al. (2015) Fungal Effectors and Plant Susceptibility. Annu Rev Plant Biol 66: 513-545. 
Richie, D.L., Hartl, L., Aimanianda, V., Winters, M.S., Fuller, K.K., Miley, M.D., et al. (2009) A role for the unfolded protein response (UPR) in virulence and antifungal susceptibility in Aspergillus fumigatus. PLoS Pathog 5: 1-17.

Ron, D., and Walter, P. (2007) Signal integration in the endoplasmic reticulum unfolded protein response. Nat Rev Mol Cell Biol 8: 519-529.

Rüegsegger, U., Leber, J.H., and Walter, P. (2001) Block of HAC1 mRNA translation by long-range base pairing is released by cytoplasmic splicing upon induction of the unfolded protein response. Cell 107: 103-114.

Sambrook, J., Frisch, E.F., and Maniatis, T. (1989) Molecular Cloning: A Laboratory Manual.

Scherer, M. (2006) The Clp1 Protein Is Required for Clamp Formation and Pathogenic Development of Ustilago maydis. Plant Cell Online 18: 2388-2401.

Schmitz, L., McCotter, S., Kretschmer, M., Kronstad, J.W., and Heimel, K. (2018) Transcripts and tumors: regulatory and metabolic programming during biotrophic phytopathogenesis. F1000 Res 7: 1-8.

Schulz, B., Banuett, F., Dahl, M., Schlesinger, R., Schäfer, W., Martin, T., et al. (1990) The $b$ alleles of $U$. maydis, whose combinations program pathogenic development, code for polypeptides containing a homeodomain-related motif. Cell 60: 295-306.

Scrimale, T., Didone, L., Mesy Bentley, K.L. de, and Krysan, D.J. (2009) The Unfolded Protein Response Is Induced by the Cell Wall Integrity Mitogen-activated Protein Kinase Signaling Cascade and Is Required for Cell Wall Integrity in Saccharomyces cerevisiae. Mol Biol Cell 20: 164-175.

Shore, G.C., Papa, F.R., and Oakes, S.A. (2011) Signaling Cell Death from the Endoplasmic Reticulum Stress Response. Curr Opin Cell Biol 34: 474-476.

Stasio, M. Di (2009) Regulation des Kpp2/Kpp6-MAPK-Moduls in Ustilago maydis durch Phosphatasen. PhD Thesis.

Stasio, M., Brefort, T., Mendoza-Mendoza, A., Münch, K., and Kahmann, R. (2009) The dual specificity phosphatase Rok1 negatively regulates mating and pathogenicity in Ustilago maydis. Mol Microbiol 73: 73-88. 
Chapter 3 | UPR-dependent inhibition of mating type signaling

Tabas, I., and Ron, D. (2011) Effects of nanomechanical bone tissue properties on bone tissue strain: Implications for osteocyte mechanotransduction. Nat Cell Biol 13: 184-190.

Tsukuda, T., Carleton, S., Fotheringham, S., and Holloman, W.K. (1988) Isolation and characterization of an autonomously replicating sequence from Ustilago maydis. Mol Cell Biol 8: 3703-3709.

Urban, M., Kahmann, R., and Bölker, M. (1996) Identification of the pheromone response element in Ustilago maydis. Mol Gen Genet 251: 31-37.

Wahl, R., Zahiri, A., and Kämper, J. (2010) The Ustilago maydis b mating type locus controls hyphal proliferation and expression of secreted virulence factors in planta. Mol Microbiol 75: 208-220.

Walter, P., and Ron, D. (2011) The Unfolded Protein Response: From Stress Pathway to Homeostatic Regulation. Science 334: 1081-1086.

Wang, R.-J., Peng, J., Li, Q.X., and Peng, Y.-L. (2017) Phosphorylation-mediated Regulatory Networks in Mycelia of Pyricularia oryzae Revealed by Phosphoproteomic Analyses. Mol Cell Proteomics 16: 1669-1682.

Zarnack, K., Eichhorn, H., Kahmann, R., and Feldbrügge, M. (2008) Pheromone-regulated target genes respond differentially to MAPK phosphorylation of transcription factor Prf1. Mol Microbiol 69: 1041-1053. 
4 Chapter 4 | Tool for conditional gene expression

\section{Novel tools for conditional gene expression enable the analysis}

of stage-specific protein functions in the Ustilago maydis/maize

\section{pathosystem}

Lara Schmitz $^{1}$, James Kronstad ${ }^{2}$, Kai Heimel ${ }^{1}$

${ }^{1}$ Georg-August-University-Göttingen, Institute for Microbiology and Genetics

Department of Molecular Microbiology and Genetics, Grisebachstr. 8

D-37077 Göttingen, Germany

${ }^{2}$ Michael Smith Laboratories, Department of Microbiology and Immunology, University of British Columbia, Vancouver, BC, Canada V6T 1 Z4

\section{Author contributions:}

LS and KH designed the study. LS carried out the experiments. LS designed the figures. LS wrote and $\mathrm{KH}$ edited the manuscript. JK provided tools and resources. 
Chapter 4 | Tool for conditional gene expression

\subsection{Abstract}

Ustilago maydis is a model organism to study biotrophic plant-pathogen interactions. Sexual and pathogenic development of the fungus are tightly connected, fusion of compatible haploid sporidia is prerequisite for infection the host plant Zea mays. After plant penetration, the unfolded protein response (UPR) is activated and required for biotrophic growth. The UPR is continuously active throughout all stages of pathogenic development in planta. However, since development of UPR deletion mutants stops directly after plant penetration, the role of an active UPR at later stages of development has/could not be examined, yet. Here, we establish a gene expression system for $U$. maydis that uses endogenous, conditionally active promoters to either induce or repress expression of a gene of interest at different stages of plant infection. Integration of the expression constructs into the native genomic locus and removal of resistance cassettes was required to obtain a wild type-like expression pattern. This indicates that genomic localization and chromatin structure are important for correct promoter activity and gene expression. By conditional expression of the central UPR regulator Cib1 in U. maydis, we show that a functional UPR is important for plant defense suppression at later stages of development and that U. maydis relies on a robust control system to prevent deleterious UPR hyperactivation.

\subsection{Introduction}

The phytopathogenic basidiomycete $U$. maydis causes the smut disease on Z. mays (maize) and is an important model organism to study basic cellular processes, such as DNA recombination and vesicular transport, but also sexual development and biotrophic fungal/plant interactions (Banuett, 1995; Kahmann and Kämper, 2004; Bakkeren et al., 2008; Dean et al., 2012; Lanver et al., 2018).

The available genome sequence and a broad range of molecular techniques and tools enable the efficient genetic manipulation of $U$. maydis (Brachmann et al., 2004; Kämper, 2004; Kämper et al., 2006; Terfrüchte et al., 2014; Schuster et al., 2016). Common and frequently used ways to characterize gene functions are deletion or overexpression of genes, as well as the generation of gene fusions for fluorescence microscopy or epitope tagging. PCR-based methods for gene replacement via homologous recombination as well as promoters for constitutive, inducible or titratable (over)expression of genes like the tef, otef, or narl, crgl or tet-Off promoter are available (Banks et al., 1993; Spellig et al., 1996; 
Brachmann, 2001; Bottin et al., 2002; Brachmann et al., 2004; Kämper, 2004; Zarnack et $a l ., 2008)$. These promoters can be fused to a gene of interest and either integrated in the native gene locus or into the locus of the succinate dehydrogenase-encoding gene (UMAG_00844) (ip locus) by homologous recombination (Keon et al., 1991). However, gene expression analysis using metabolism-dependent promoters may result in pleiotropic effects due to metabolic changes and unwanted overexpression of the gene of interest. Other conditional gene expression systems in fungi include for example estrogen-, orzearalenone-, or light-inducible expression systems for Aspergillus sp. (Pachlinger et al., 2005), Gibberella zeae (Lee et al., 2010), or Neurospora crassa (Salinas et al., 2018), respectively (for comprehensive overview see: Kluge et al., 2018). These systems are all suitable to control gene expression in axenic culture. However, tools to specifically address the function of genes during organismal interactions, such as fungal/plant interactions, are not well established, yet.

U. maydis is a dimorphic fungus, specifically infecting its host plant maize. Sexual and pathogenic development are interconnected processes, since plant infection requires cell/cell fusion of compatible haploid sporidia to generate the infectious, dikaryotic filament. Accordingly, mating, filamentous growth and plant penetration are controlled by a tetrapolar mating-type system (Hartmann et al., 1996b; Bölker, 2001; Feldbrügge et al., 2004). The $a$-mating type locus encoded pheromone-receptor system regulates cell-cell recognition and fusion (Bölker et al., 1992), whereas all subsequent steps of pathogenic development are controlled by the $b$-mating type locus encoded bE/bW-heterodimer (Schulz et al., 1990; Kämper et al., 1995; Heimel et al., 2010b; Wahl et al., 2010b). After penetration of the plant surface, $U$. maydis establishes a compatible biotrophic interaction with the host plant via secretion of effectors that suppress plant defense reactions (Lo Presti et al., 2015a; Lanver et al., 2018). Typically, expression of effector encoding genes is plant-specifically induced (Kämper et al., 2006; Lanver et al., 2018), resulting in increased stress imposed on the endoplasmic reticulum (ER). Activation of the unfolded protein response (UPR) is critical to counteract elevated ER stress levels and required for efficient secretion of effector proteins (Lo Presti et al., 2015b; Hampel et al., 2016; Pinter et al., 2019). ER stress is sensed by the ER-membrane spanning kinase/RNase Ire1. Ire1 activity enables unconventional cytoplasmic splicing and the subsequent translation of the mRNA encoding Hac1 in Saccharomyces cerevisiae, XBP1 in higher eukaryotes and Cib1 in U. maydis (Cox and Walter, 1996; Kawahara et al., 1998; Rüegsegger et al., 2001; Heimel 
Chapter 4 | Tool for conditional gene expression

et al., 2013). In U. maydis, the UPR is required for pathogenicity and active throughout all stages of biotrophic growth inside the host plant. However, since growth of cibl mutant strains is $2 q$

arrested early after plant infection, the importance of a functional UPR for later stages of biotrophic development in planta has not been addressed, yet.

Here, we describe a new approach for conditional and stage-specific gene expression during plant infection of $U$. maydis. Based on previously published transcriptome data of fungal gene expression during biotrophic growth, genes with desired in planta expression patterns were identified and their promoters were used for conditional gene expression. Importantly, maintenance of the genomic context and removal of resistance cassettes is prerequisite to facilitate correct promoter activity and expression pattern. As a proof of principle, we used different conditional promoters to address the function of the UPR regulator Cib1 and the effect of stage-specific overexpression at later stages of biotrophic development. We demonstrate that a functional UPR is not only essential for establishment of biotrophy, but also important at later stages of development in planta.

\subsection{Results}

\subsubsection{Identification of conditional promoters}

Aiming for a system that allows gene expression with a defined pattern, candidate genes expressed during early biotrophic development of $U$. maydis were identified. Using RNAseq data published by Lanver et al., 2018, we identified the genes $U M A G_{-} 12184$ and $U M A G \_03597$. Both genes are expressed in axenic culture and at early stages of pathogenic development, but considerably reduced in expression two (UMAG_12184) or four days post inoculation (dpi, UMAG_03597) (Figure 4.1A). To test if these genes are involved in virulence, the haploid, solopathogenic strain SG200 and derivatives deleted for either $U M A G \_12184$ or UMAG_03597 were tested in plant infection experiments. SG200 expresses a compatible $\mathrm{bE} / \mathrm{bW}$-heterodimer, and thus is capable of forming filaments and infecting its host plant, Z. mays, without the need of a compatible mating partner (Kämper et al., 2006). Deletion strains were not affected in virulence (Figure 4.1B), demonstrating that these genes are dispensable for pathogenic development. When spotted on charcoal containing solid media, SG200 forms white fuzzy colonies indicating filamentous growth. Consistent with results obtained in the plant infection experiment, SG200DUMAG_12184 


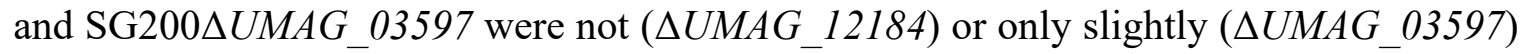
affected in filament formation (Suppl. Figure 4.1). Since SG200 $U U M A G \_03597$ showed full virulence, this phenotype does not impair the fungus' ability to infect its host. Additionally, none of the deletion strains showed increased sensitivity towards ER or cell wall stress (Suppl. Figure 4.1). Based on these results, the chosen genes were regarded as suitable candidates to facilitate conditional gene expression.

A
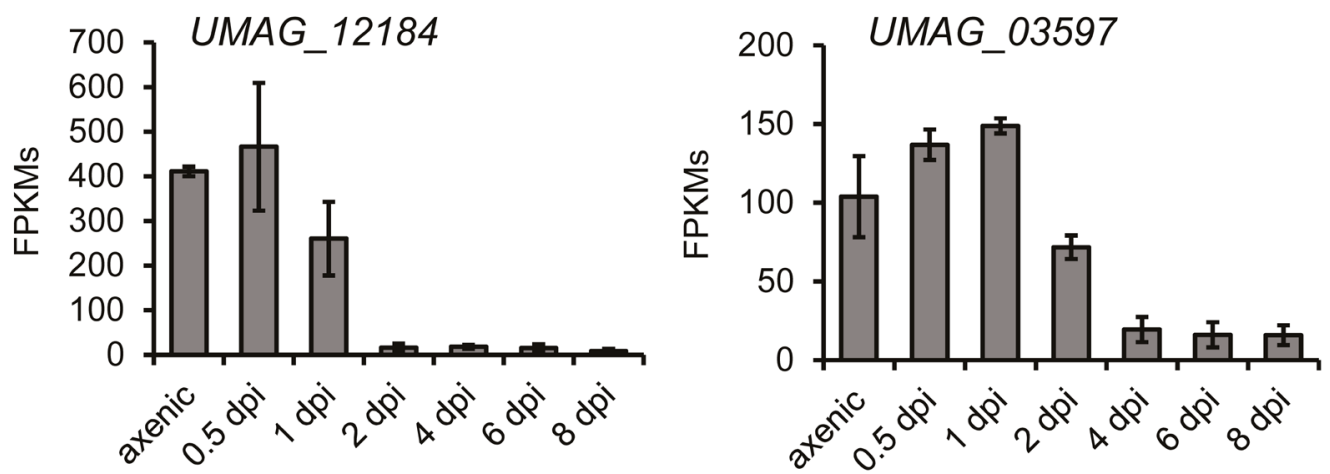

B

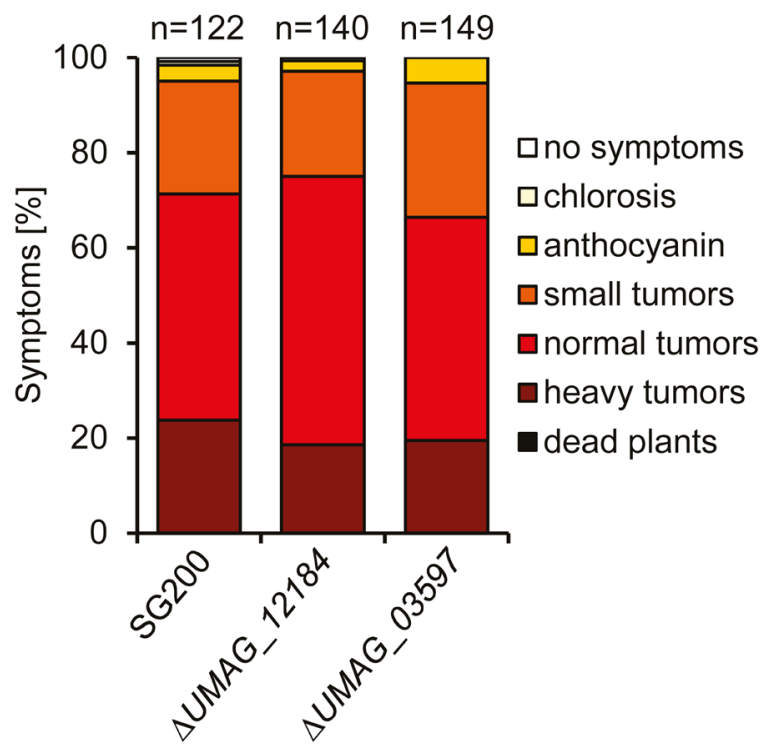

Figure 4.1 Identification and testing of promoters for conditional gene expression. (A) Fragments Per Kilobase Million (FPKMs) of the $U M A G_{-} 12184$ and $U M A G_{-} 03597$ genes up to 8 days post inoculation (dpi). 6 day-old maize seedlings were injected with a mixture of compatible haploid strains FB1 and FB2 and plant material was harvested at the indicated time points. Raw data was extracted from Lanver et al., 2018. (B) Plant infection assay with the solopathogenic strain SG200 and derivatives. SG200, SG200_UMAG_12184 and SG200AUMAG_03597 were inoculated into $8 \mathrm{~d}$-old maize seedlings. Disease symptoms were rated 8 days after inoculation (dpi) and grouped into categories as shown in the figure legend. $\mathrm{n}=$ number of infected plants. 
Chapter 4 | Tool for conditional gene expression

\subsubsection{Cib1 is required throughout biotrophic development in planta}

cibl encodes the central regulator of the UPR in U. maydis coordinating pathogenic development and efficient secretion of effectors to suppress the plant defense response (Heimel et al., 2013). In cibl deletion strains, fungal development stops directly after plant penetration and virulence is strongly attenuated, only leading to formation of chlorosis but not tumors (Heimel et al., 2013). To test if cibl is only important directly after plant penetration (release of the cell cycle block and establishment of the biotrophic interaction), or if it is necessary also at later stages of pathogenic development, we expressed cibl under control of promoters of $U M A G_{-} 12184$ and $U M A G_{-} 03597$ (shut off at 2 and 4 dpi, respectively). To this end, cibl was integrated into the native genomic loci of $U M A G \_12184$ and $U M A G \_03597$, respectively, in strain FB2 $\Delta$ cibl (Heimel et al., 2010a). Thereby $U M A G \_12184$ or $U M A G \_03597$ was replaced with the cibl open reading frame (ORF), generating strains FB2 $\triangle$ cibl $\triangle U M A G_{-} 12184: \because c i b 1$ and FB2 $\Delta c i b 1$ $\triangle U M A G \_03597:: c i b 1$.

The generated strains were tested for correct expression of cibl by analysis of ER stress resistance using Tunicamycin (TM) or dithiothreitol (DTT). Both mutants showed ER stress resistance similar to the WT (FB2) control, demonstrating that cibl expression driven by the conditional promoters is sufficient to suppress the ER-stress hypersensitivity of the FB2 $\Delta$ cib1 progenitor strain (Figure 4.2A) (Heimel et al., 2013). Additionally, when compatible mixtures of FB1, FB2, FB1 $\Delta c i b 1, \mathrm{FB} 2 \Delta c i b 1$ and derivatives FB2 $\Delta c i b 1$ $\triangle U M A G_{-} 12184:: c i b 1$ or FB2 $\triangle$ cibl $\triangle U M A G \_03597: \because c i b 1$ were spotted on charcoal containing media (Figure 4.2B), all tested combinations developed white fuzzy colonies (Banuett and Herskowitz, 1989), indicating that mating is not affected.

In plant infection assays, symptom development after inoculation of compatible mixtures of FB1 $\Delta c i b 1 \times$ FB2 were similar to the FB1 xFB2 (WT) control (Figure 4.2C), demonstrating that one functional copy of cibl is sufficient for full virulence of the fungus. However, when cibl was expressed under the control of $\mathrm{P}_{U M A G_{-}} 12184$ (FB2 $\Delta c i b 1$ $\left.\triangle U M A G_{-} 12184: \because c i b 1\right)$, virulence was strongly attenuated, resembling the $\Delta c i b 1$ phenotype. By contrast, cibl expression under the control of $\mathrm{P}_{U M A G_{-} 03597}$ (FB2 $\Delta c i b 1$ $\left.\triangle U M A G \_03597:: c i b 1\right)$ lead to formation of anthocyanin and small tumors, indicating further progression of fungal development in planta. 
A

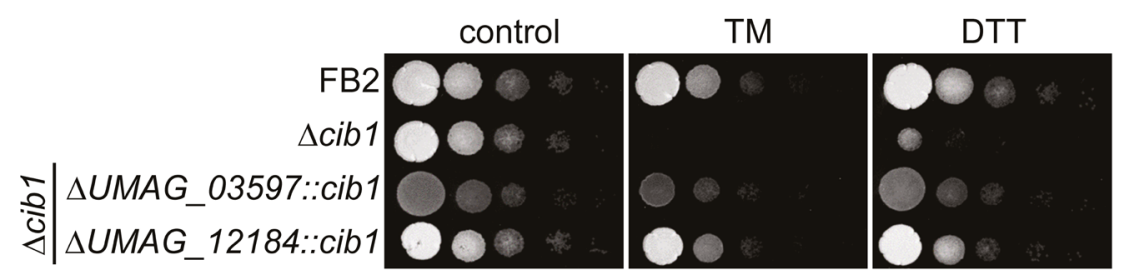

B

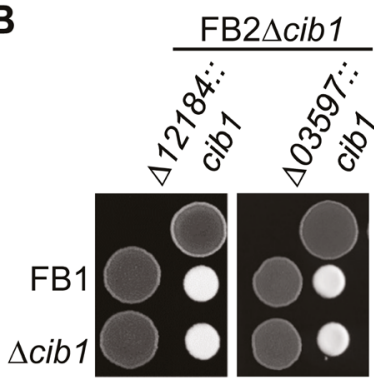

C
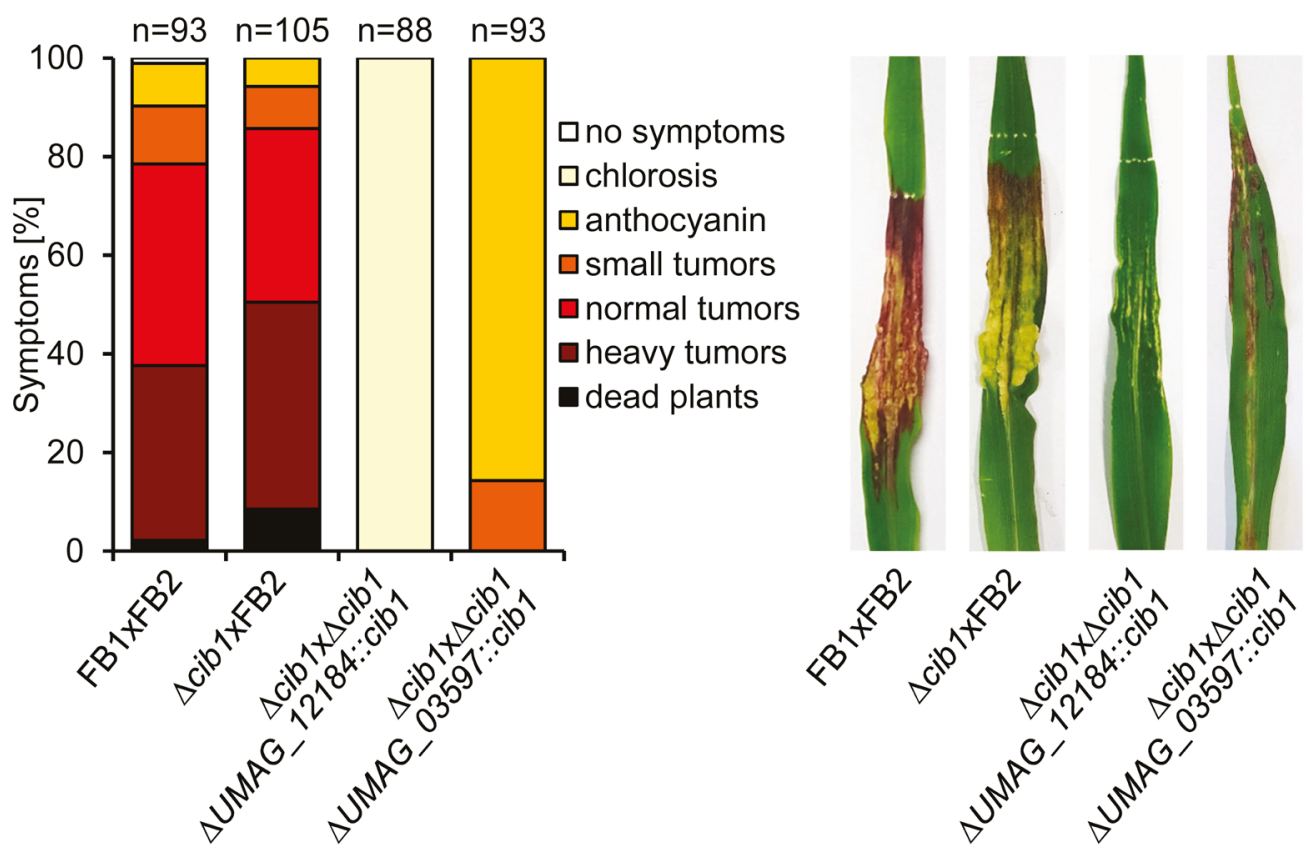

Figure 4.2 Conditional cib1 expression restores ER-stress resistance, but not pathogenicity. (A) ER stress assay of strains FB2 (WT), FB2 $\Delta c i b 1$, and derivatives. Serial 10-fold dilutions were spotted on YNBG solid medium supplemented with TM $(0.5 \mu \mathrm{g} / \mathrm{mL})$ or DTT $(0.5 \mathrm{mM})$. Pictures were taken after 48 hours of incubation at $28{ }^{\circ} \mathrm{C}$. (B) Mating assay with FB1, FB1 $\Delta c i b 1$ and FB2 $\Delta c i b 1 \Delta U M A G \_12184: \because c i b 1$ and FB2 $\triangle$ cibl $\triangle U M A G \quad 03597:: c i b 1$. Compatible mixtures of strains were spotted on potato dextrose solid media supplemented with $1 \%$ charcoal. Photos were taken after 24 hours at $28^{\circ} \mathrm{C}$. White fuzzy colonies indicate the formation of filaments. (C) Plant infection assay with FB1 and FB2, FB1 $\Delta c i b 1$ and FB2, FB2 $\Delta c i b 1$ $\triangle U M A G \_12184: \because c i b 1$ and FB2 $\triangle c i b 1 \triangle U M A G \_03597:: c i b 1.8$ d-old maize seedlings were co-inoculated with compatible strain mixtures as indicated in the figure. Disease symptoms were rated 8 dpi and grouped into categories as shown in the figure legend. $\mathrm{n}=$ number of inoculated plants. Pictures of leaves were taken at $8 \mathrm{dpi}$ and represent the most common infection symptom. Significance was calculated using the Mann-Whitney-test. ${ }^{*} \mathrm{P}$ value $<0.05,{ }^{* *} \mathrm{P}<0.01$, and ${ }^{* * * \mathrm{P}}<0.001$.

To assess at which step biotrophic development of the fungus stopped, infected leaves were harvested at 2, 4 and 6 dpi and stained with Chlorazol Black E (Figure 4.3A). Microscopic analysis revealed extensive proliferation and clamp cell formation when plants were inoculated with combinations of FB1xFB2 or FB1xFB2 $\triangle$ cibl WT strains. By contrast, cells expressing cibl under the control of $P_{U M A G_{-} 12184}$ (FB1 $\Delta$ ciblxFB2 $\Delta c i b 1$ $\left.\triangle U M A G_{-} 12184:: \mathrm{cibl}\right)$ formed filaments and penetrated the plant surface via appressoria (2 dpi). However, pathogenic development did not progress further. Subsequent steps of 
Chapter 4 | Tool for conditional gene expression

pathogenic development, including clamp cell formation and extended fungal proliferation was not observed. By contrast, $\mathrm{P}_{U M A G_{-} 03597: c i b 1}$ (FB2 $\triangle$ cibl $\triangle U M A G_{-}$03597::cib1) expression enabled the fungus to induce proliferation, hyphal branching and clamp cell formation in planta. Importantly, proliferation was still reduced compared to the controls (FB1xFB2 and FB1 $\Delta c i b 1 \times$ FB2).

Previous studies revealed that plants inoculated with $\Delta c i b 1$ mutant strains showed increased plant defense reactions as evident by elevated expression of pathogenesis related $(P R)$ genes at 2 dpi (Heimel et al., 2013). It is conceivable, that this observation is related to the requirement of a functional UPR for efficient processing and secretion of effectors (Lo Presti et al., 2015b; Hampel et al., 2016; Pinter et al., 2019). To test if Cib1 is also required to suppress the plant defense at later stages, we determined expression levels of $P R 1, P R 3$ and $P R 5$ at 2, 4 and 6 dpi in plants inoculated with strains conditionally expressing cibl. All three $P R$ genes are markers for salicylic acid (SA)-related defense responses that are typically suppressed by biotrophic plant pathogens like $U$. maydis (Glazebrook, 2005). Consistent with the results obtained in infection studies,

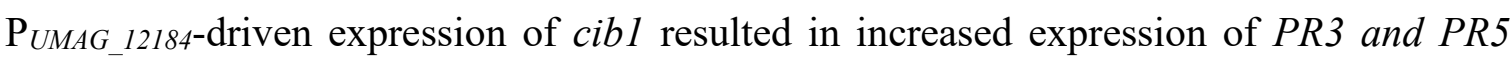
genes at 2 dpi, whereas expression of PRl was not induced (Figure 4.3B). By contrast, when cibl was expressed under the control of $\mathrm{P}_{U M A G_{-} 03597,} P R$ gene expression was significantly induced at $6 \mathrm{dpi}$, although $P R 3$ and $P R 5$ levels were already elevated at earlier time points. Hence, our data indicate that cibl expression under control of the promoter of $U M A G_{-} 12184$ is not sufficient to establish a compatible biotrophic interaction in planta leading to a block in pathogenic development. By contrast, when cibl is expressed for an extended time (PUMAG_03597), a compatible interaction appears to be established, allowing further proliferation. This suggests that $c i b 1$ is required for plant defense suppression not only at the onset, but also later stages of biotrophic development in planta. 
A

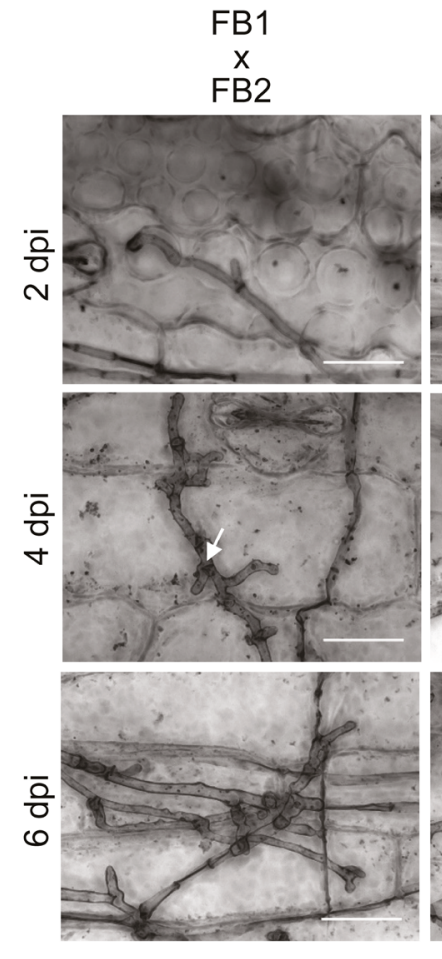

$\Delta c i b 1$
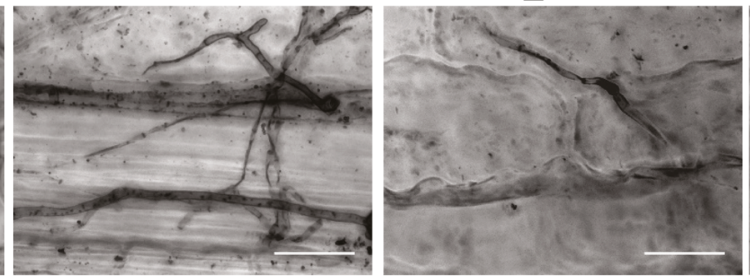

B
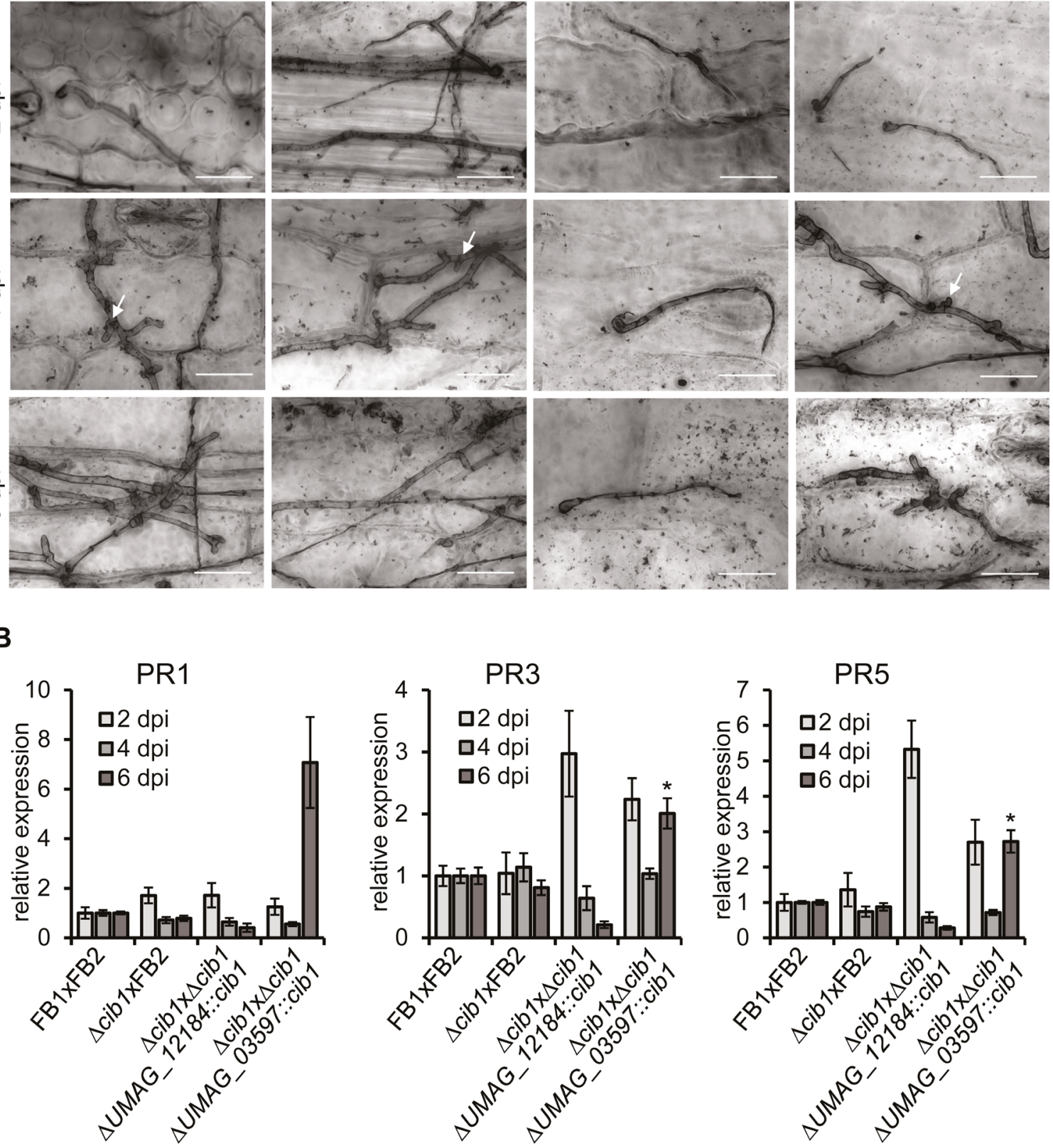

Figure 4.3 Analysis of fungal morphology and plant defense response of conditional cib1 mutant strains. (A) Fungal proliferation of compatible mixtures of FB1 and FB2, FB1 $\Delta c i b 1$ and FB2, FB2 $\Delta c i b 1$ $\triangle U M A G_{-} 12184: \because c i b 1$ or FB2 $\Delta c i b 1 \quad \triangle U M A G_{-} 03597: \because c i b l$ investigated by Chlorazol Black E staining of infected leaf samples at 2, 4 and $6 \mathrm{dpi}$. Arrows mark clamp cells indicative of fungal proliferation in planta. Scale bar $=20 \mu \mathrm{m}$. (B) qRT-PCR analysis of $P R 1, P R 3$ and $P R 5$ gene expression of infected maize leaves at 2, 4 and 6 dpi. Maize seedlings were inoculated with the indicated strains. GAPDH was used for normalization. Expression values represent the mean of three biological replicates with two technical duplicates each. Error bars represent the SEM. Statistical significance was calculated using the students $t$ test. $* \mathrm{P}$ value $<0.05, * * \mathrm{P}<0.01$, and $* * * \mathrm{P}<0.001$ 
Chapter 4 | Tool for conditional gene expression

\subsubsection{Genomic localization and presence of a resistance cassette affects gene expression levels}

The mig2 gene cluster contains five highly homologous genes which are plant-specifically induced but not involved in virulence of $U$. maydis (Basse et al., 2002). The five mig2 genes (mig2_1, mig2_2, mig2_3, mig2_4 and mig2_5) differ in their expression strength and time of activation. Thus, their promoters represent suitable targets for controlled and plant-specific expression of a gene of interest. To assess the effect of overexpressing the spliced version of the $c i b 1 \mathrm{mRNA}$ ( $c i b 1^{\mathrm{s}}$ in the following), encoding the UPR regulator Cib1, during pathogenic development in planta, we integrated a $\mathrm{P}_{\text {mig2 } 1:}: \mathrm{Cibl}^{\mathrm{s}}$ promoter fusion into the ip locus of SG200. The ip or $c b x$ locus is commonly used for integration of linear DNA in $U$. maydis by homologous recombination, conferring resistance against carboxin (Brachmann, 2001). Since virulence of strain SG200P mig2_l $_{2}: \mathrm{cibl}^{\mathrm{S}}$ was severely attenuated in plant infection experiments (Figure 4.4A), we investigated at which stage pathogenic development was blocked. This revealed the inability of $\mathrm{SG}_{200 \mathrm{P}_{\text {mig2 }} \text { : }}: \mathrm{cibl}^{\mathrm{s}}$ to induce filamentous growth on charcoal containing solid media and on the leaf surface (Figure 4.4B).

Since previous studies showed that constitutive expression of $c i b 1^{\mathrm{s}}$ inhibits the formation of infectious filaments (Heimel et al., 2013), we checked expression levels of $c i b 1^{\mathrm{s}}$ in SG200P $P_{\text {mig2 }}{ }_{1}: c i b 1^{\mathrm{s}}$ in axenic culture. This revealed increased levels of $c i b 1^{\mathrm{s}}$ in strain SG200P mig2 $_{2}:$ cibl $^{s}$, although the mig2_1 promoter is considered to be "plant-specific". Since elevated $c i b 1^{\mathrm{s}}$ levels might result from increased activity of the $c i b 1$ WT ORF that is

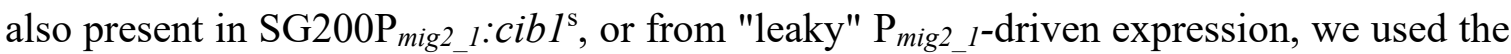
$\Delta c i b 1$ background for further analyses. To study if this effect might be specific for the ip locus, we integrated $c i b 1^{\mathrm{s}}$ into the mig2_l locus, replacing the mig2 1 ORF, generating U. maydis strain FB1 $\Delta c i b 1 \Delta m i g 2 \_1:: c i b 1^{\mathrm{s}}\left(\right.$ mig2 $_{1} 1$ locus $\left.\left(+\mathrm{Nat}^{\mathrm{R}}\right)\right)$. To exclude potential effects of the resistance cassette used for integration, the nourseothricin $\left(\mathrm{Nat}^{\mathrm{R}}\right)$ resistance cassette was removed by FLP/FRT recombination (Khrunyk et al., 2010). This revealed that elevated $c i b 1^{\text {s}}$-levels indeed resulted from $\mathrm{P}_{m i g 2}{ }_{2}$ activity and only strains in which the nourseothricin resistance marker (mig2_l locus $\left(-\mathrm{Nat}^{\mathrm{R}}\right)$ ) was removed, were devoid of any detectable $c i b 1^{\text {s }}$ expression (Figure 4.4C). In summary, our data strongly suggest that both, the place of integration and the presence of a resistance marker contribute to the increased activity of the mig2_1 promoter in axenic culture. 
To pinpoint if this effect is specific for $c i b 1^{\text {s }}$, we performed an analogous experiment with the pit 1 and pit 2 genes, both of which are divergently transcribed from the same promoter. Under normal conditions, expression of both genes is barely detectable in axenic culture, but highly induced during biotrophic growth in planta (Doehlemann et al., 2011; Lanver et al., 2018). We determined expression levels of both genes when 1) (re-)integrated in the ip locus or their native locus, using either 2) $\mathrm{Nat}^{\mathrm{R}}$ or 3 ) hygromycin resistance $\left(\mathrm{Hyg}^{\mathrm{R}}\right)$ cassettes and 4) after removal of the resistance marker (Figure 4.4D). Transcript levels of both, pit1 and pit2, were drastically increased (approximately 400-fold and 800-fold, respectively) compared to the SG200 (WT) control when integrated in the ip locus. When re-integrated into the native locus, expression was still significantly increased (pit1: 49-fold ( $\mathrm{Nat}^{\mathrm{R}}$ ) and 10-fold $\left(\mathrm{Hyg}^{\mathrm{R}}\right)$; pit2: 134-fold $\left(\mathrm{Nat}^{\mathrm{R}}\right)$ and 13-fold $\left.\left(\mathrm{Hyg}^{\mathrm{R}}\right)\right)$ and only after recycling of the resistance marker (pit1/2 locus $\left(-\mathrm{Hyg}^{\mathrm{R}}\right)$ ) expression was similar to the WT control. Taken together, these data show that the locus of integration and presence of a resistance cassette can drastically influence gene expression levels. 
Chapter 4 | Tool for conditional gene expression

A

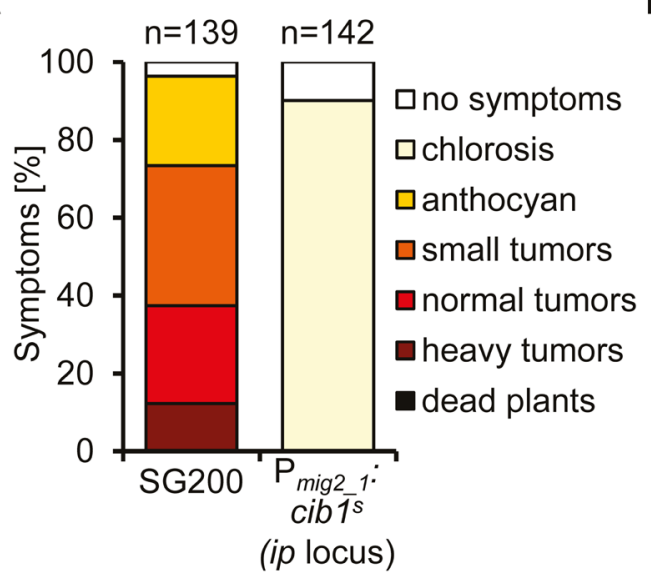

B

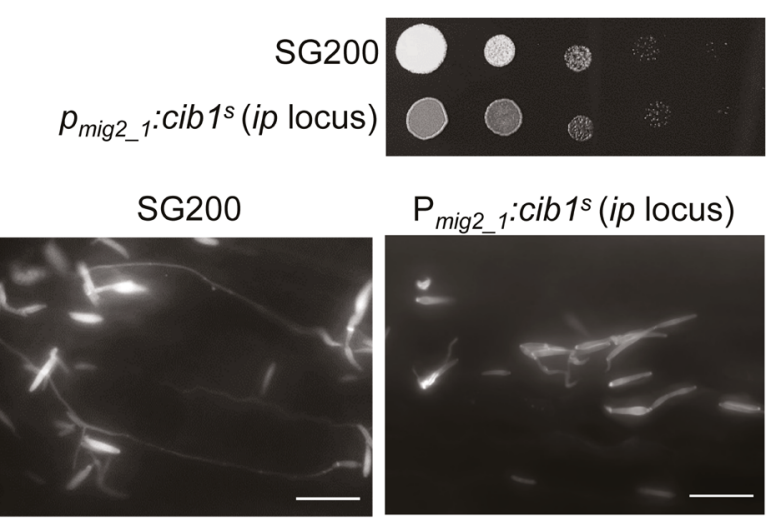

C
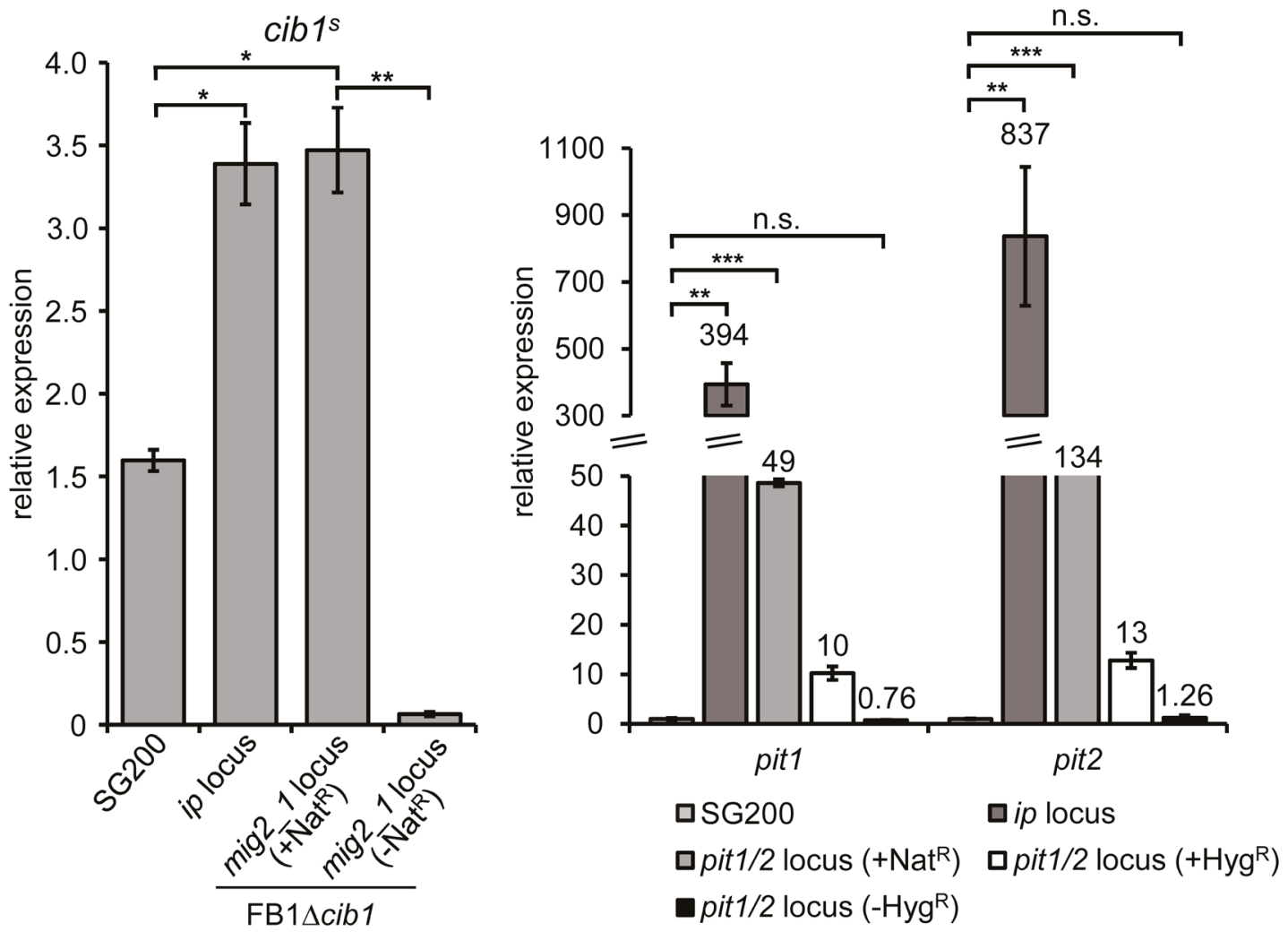
Figure 4.4 The locus of integration and presence of a resistance cassette influence gene expression. (A) Plant infection assay with the solopathogenic strain SG200 and derivative. SG200 and SG200 $\mathrm{P}_{\text {mig2 }_{-}}$:cib1 $^{\mathrm{s}}$ (ip locus) were inoculated into $8 \mathrm{~d}$-old maize seedlings. Disease symptoms were rated $8 \mathrm{dpi}$ and grouped into categories as shown in the figure legend. $\mathrm{n}=$ number of infected plants. (B) Analysis of $b$-dependent filament formation on PD-CC solid media and on the leaf surface. SG200 and SG200 $\mathrm{P}_{\text {mig2 }}$ : cibl $^{\text {s }}$ (ip locus) were spotted on PD-CC solid media. Photos were taken after 24 hours at $28^{\circ} \mathrm{C}$. White fuzzy colonies indicate the formation of filaments. Fungal hyphae were stained 24 hours after inoculation with calcofluor to visualize the fungal hyphae. Scale bar $=10 \mu \mathrm{m}$. (C) qRT-PCR analysis of $c i b 1^{\text {s }}$ gene expression when integrated in different loci and after removal of the resistance cassette. Primers specifically detecting the spliced cibl transcript were used. RNA was isolated from exponentially growing U. maydis strains SG200, SG200 $\mathrm{P}_{\text {mig2_l }}:$ cibl $^{\mathrm{s}}$ (ip locus integration), FB1 $\Delta c i b 1 \Delta m i g 2 \_1:: \mathrm{Pcibl}^{\mathrm{s}}$ (mig2_1 locus, +Nat ${ }^{\mathrm{R}}$ ) and FB1 cibl $\Delta m i g 2 \_1:: c i b 1^{\mathrm{s}}\left(\right.$ mig2 $_{-} 1$ locus, $\left.-\mathrm{Nat}{ }^{\mathrm{R}}\right) . e I F 2 b$ was used for normalization. Expression values represent the mean of three biological replicates with two technical duplicates each. Error bars represent the SEM. Statistical significance was calculated using the students $t$ test. $* \mathrm{P}$ value $<0.05, * * \mathrm{P}<0.01$, and $* * * \mathrm{P}<0.001$. (D) qRT-PCR analysis of pit1 and pit 2 gene expression when integrated in different loci and after removal of the resistance cassette. RNA was isolated from exponentially growing U. maydis strains SG200, SG200

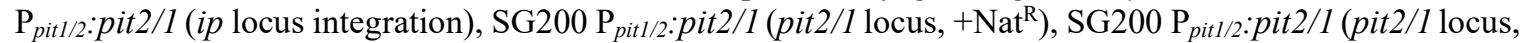
$+\mathrm{Hyg}^{\mathrm{R}}$ ) and $\mathrm{P}_{\text {pit } 1 / 2}:$ pit2/1 (pit2/1 locus, $-\mathrm{Hyg}^{\mathrm{R}}$ ). eIF2b was used for normalization. Expression values represent the mean of three biological replicates with two technical duplicates each. Error bars represent the SEM. Statistical significance was calculated using the students $t$ test. $* \mathrm{P}$ value $<0.05, * * \mathrm{P}<0.01$, and $* * * \mathrm{P}<0.001$.

\subsubsection{Overexpression of $c i b 1^{\text {s }}$ does not disturb pathogenic development in planta}

The UPR is specifically induced after plant penetration and remains active throughout development in planta (Heimel et al., 2010a; Heimel et al., 2013). Activity of the UPR is controlled on posttranscriptional level by Ire1-mediated unconventional splicing (Heimel et al., 2013). However, it is conceivable that long-term UPR activity in planta requires efficient mechanisms to modulate the UPR and counteract deleterious hyperactivation (Heimel et al., 2013; Pinter et al., 2019). To specifically increase $c i b l^{\mathrm{s}}$ levels in planta and address the effect of UPR hyperactivation on pathogenic development, we expressed $c i b 1^{\mathrm{s}}$ under control of the migl and mig2_l promoter. To this end, the mig1 or mig2_l ORFs were replaced by $c i b 1^{\text {s }}$, followed by the removal of the resistance cassette in U. maydis strain FB1 $\Delta c i b 1$ (Heimel et al., 2010a). When spotted on solid media supplemented with TM or DTT, the hyper-susceptibility of the FB1 $1 \Delta c i b 1$ progenitor strain was not suppressed, demonstrating that both, $\mathrm{P}_{\text {migl }}$ and $\mathrm{P}_{\text {migz_l }}$, are not active in axenic culture (Figure 4.5A). Consistently, mating was not affected (Figure 4.5B) in any of the tested combinations.

Compatible mixtures of strains FB1, FB2, FB1 $\Delta c i b 1, \mathrm{FB} 2 \Delta c i b 1$, and the derivatives $\Delta m i g 1:: c i b 1^{\mathrm{s}}$ and $\Delta m i g 2 \_1:: c i b 1^{\mathrm{s}}$ were used for plant infection studies (Figure 4.5C). $\mathrm{P}_{\text {migl }}$-or $\mathrm{P}_{\text {migz_l }}$-mediated overexpression of $c i b 1^{\mathrm{s}}$ did not affect pathogenicity when strains were combined with the compatible FB2 WT strain. This indicates that the mechanisms to prevent UPR hyperactivation in planta are robust and efficient in U. maydis. This is consistent with previous results, demonstrating that constitutive expression of $c i b 1^{\mathrm{s}}$ negatively affects pathogenic development before plant penetration, but not in planta 
Chapter 4 | Tool for conditional gene expression

(Heimel et al., 2013). Interestingly, when $\Delta m i g 1:: c i b 1^{\mathrm{s}}$ and $\Delta m i g 2 \_1:: c i b 1^{\mathrm{s}}$ strains were co-inoculated with the compatible FB2 $\Delta c i b 1$ strain, virulence was reduced, but significantly higher in comparison to the FB1 $\Delta c i b 1 \times \mathrm{xB} 2 \Delta c i b 1$ control. This is in line with previous investigations, confirming the assumption that the UPR is specifically required during biotrophic development in planta (Heimel et al., 2010a; Heimel et al., 2013). 
A
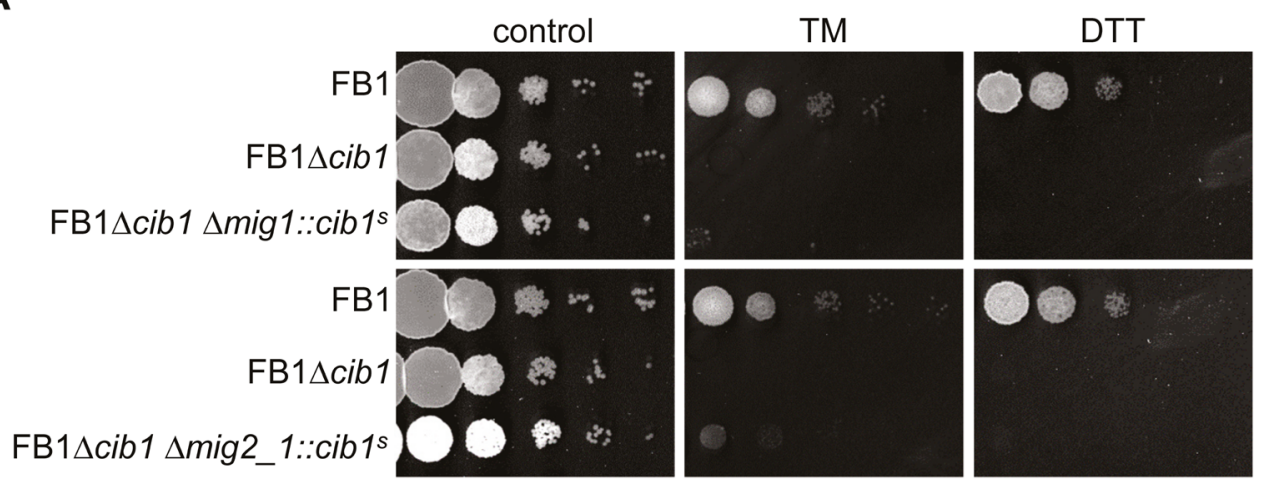

B

FB2

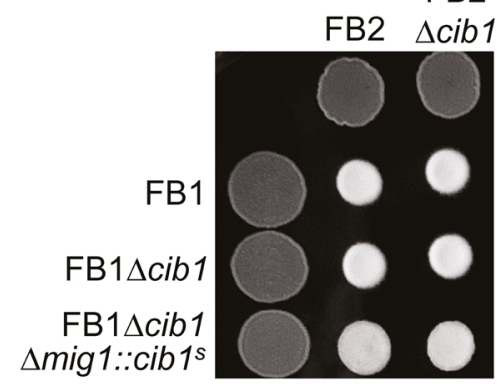

FB2

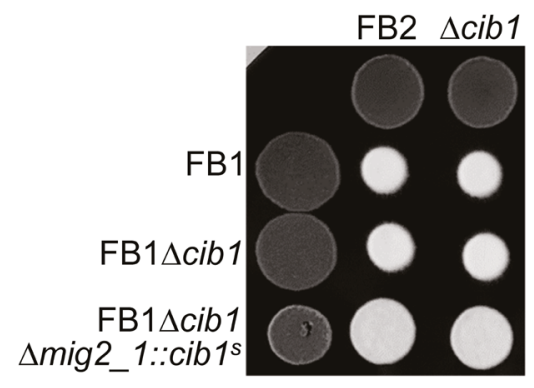

C

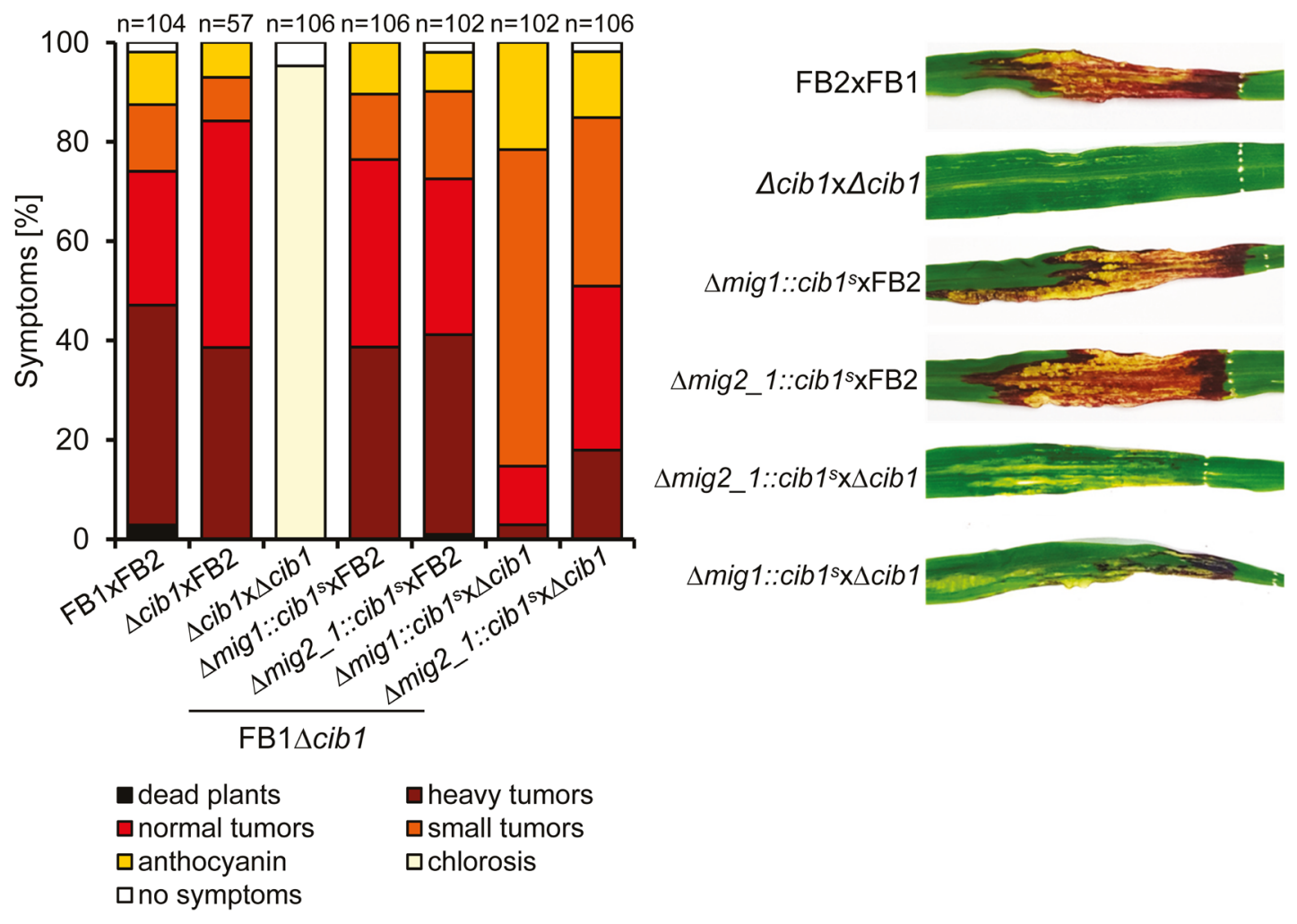


Chapter 4 | Tool for conditional gene expression

Figure 4.5 Overexpression of $\boldsymbol{c i b 1}^{\mathrm{s}}$ in planta does not affect pathogenicity of $\boldsymbol{U}$. maydis. (A) ER stress

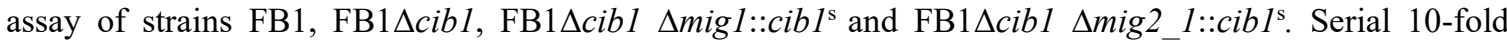
dilutions were spotted on YNBG solid medium supplemented with TM $(2.0 \mu \mathrm{g} / \mathrm{mL})$ or DTT $(1 \mathrm{mM})$. Pictures were taken after 48 hours of incubation at $28{ }^{\circ} \mathrm{C}$. (B) Mating assay with compatible mixtures of FB1, FB2, FB1 $\Delta c i b 1$, FB2 $\Delta c i b 1$, FB1 $\Delta c i b 1 \Delta m i g 1:: c i b 1^{\text {s }}$ and FB1 1 cibl $\Delta m i g 2 \_1:: c i b 1^{\text {s. }}$. Mixtures were spotted on PC$\mathrm{CC}$ solid media as shown in the figure. Photos were taken after 24 hours at $28^{\circ} \mathrm{C}$. White fuzzy colonies indicate the formation of filaments. (C) Plant infection assay with compatible mixtures of FB1 and FB2, FB1 $\Delta c i b 1$, FB2 $\Delta c i b 1$, FB1 $\Delta c i b 1 \Delta m i g 1:: c i b 1^{\text {s }}$ and FB1 $\Delta c i b 1 \Delta m i g 2 \_1:: c i b 1^{\text {s }} .8$ d-old maize seedlings were co-inoculated with the indicated strain mixtures. Disease symptoms were rated $8 \mathrm{dpi}$ and grouped into categories as shown in the figure legend. $n=$ number of inoculated plants. Pictures of leaves were taken at 8 dpi and represent the most common infection symptom.

\subsection{Discussion}

Analysis of gene functions typically involves the generation of gene deletion and overexpression strains. To test for functions related to the virulence of plant pathogenic fungi, deletion strains are inoculated into the host plant and scored for development of disease symptoms (Dean et al., 2012). However, the analysis of virulence factors that are essential for pathogenic development relies on the description of the first phenotype that is observed, e.g. the stage when pathogenic development is blocked. Hence, potential functions of these factors important at later stages of pathogenic development have not been addressed and remain elusive. Suitable tools to approach this have, to the best of our knowledge, not been developed, yet.

In this study, we report a conditional gene expression system for $U$. maydis that enables the study of gene functions at different stages of pathogenic development in the plant. We identified suitable promoters that are active during axenic growth and repressed during pathogenic growth in planta. We furthermore demonstrate that promoters (e.g. $\mathrm{P}_{\text {mig2_l }}$ or

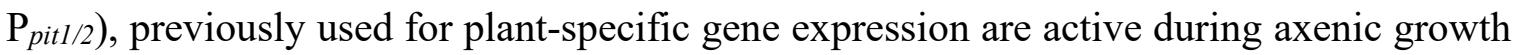
and produce considerable amounts of transcripts (up to 800-fold induced expression for pit2) when integrated into the ip locus or resistance cassettes are located in the vicinity. Only integration into the native genomic locus of mig $2 \_1$ or pit1/2, respectively, and removal of the resistance marker restored gene expression to a basal level. Similar to the mig2 gene cluster, the virulence factors pit1 and pit2 are part of a gene cluster that is specifically upregulated in planta (Basse et al., 2002; Doehlemann et al., 2011). Interestingly, gene expression of both clusters is induced in strains deleted for the histone deacetylase hdal (Reichmann et al., 2002a; Treutlein, 2007). Since Hdal regulates gene expression via deacetylation of histones (Reichmann et al., 2002b), we speculate that both gene clusters are subject to epigenetic regulation. Chromatin-based regulation of effector 
genes appears to be a common feature in plant pathogenic fungi (Soyer et al., 2014). Although the underlying molecular details remain to be addressed, it is tempting to speculate that de-repression results from alterations of the chromatin structure. It is well established that the RNA polymerase II complex is closely interacting with histone modifying enzymes, including the SWI/SNF complex and histone acetyltransferases (Wittschieben et al., 1999; Wittschieben et al., 2000). This complex is supposed to function as a chromatin snowplow leading to increased accessibility of the genomic neighborhood (Barton and Crowe, 2001). Hence, high expression of the sdh2 gene (ip locus), or of resistance marker genes might de-repress silent promoters in their vicinity.

The conditional overexpression of $\mathrm{cibl}^{\mathrm{s}}$ using the migl or mig2_l promoter did not result in alterations of disease symptoms. Since especially the migl promoter is highly active in planta (Basse et al., 2000; Lanver et al., 2018), it is remarkable that high levels of $c i b 1^{\mathrm{s}}$ are not detrimental for fungal proliferation in planta. This suggests that $U$. maydis established effective control mechanisms to prevent UPR hyperactivation, one of which is based on the functional modification of the UPR by the Cib1/Clp1 interaction, providing ER stress hyper-resistance of Clp1 expressing strains (Heimel et al., 2013; Pinter et al., 2019). A potential second mechanism might be reminiscent to UPR regulation in higher eukaryotes and involves the unspliced cibl transcript, or the encoded $\mathrm{Cib} 1^{\mathrm{u}}$ protein (Heimel et al., 2013). In higher eukaryotes, the U-isoform of the Hac1-like UPR regulator XBP1 functions as a repressor of the UPR (Yoshida et al., 2006). Hence, a similar mode of action would allow to counteract increased $c i b 1^{\mathrm{s}}$ levels, as expression of the unspliced $c i b 1$ transcript itself is subject to Cib1-dependent gene regulation.

The increasing body of transcriptomic data provides a highly valuable treasure box to identify promoters with desired expression dynamics. In theory, this enables establishment of tailor-made expression systems and address gene specific functions in a sophisticated manner. However, our attempt to identify promoters that are constantly active during axenic growth, but strongly repressed at different stages of pathogenic development in planta revealed only a low number of candidates. Moreover, we observed that it is desirable to maintain the genomic context for correct function. Using Cib1, an essential virulence factor in U. maydis, we provide a proof-of-principle and demonstrate that a functional UPR is not only required directly after penetration of the leaf surface (Heimel et al., 2010a; Heimel et al., 2013), but also at later stages of pathogenic development. The increased 
Chapter 4 | Tool for conditional gene expression

expression of $P R$ genes strongly suggests that continuous suppression of the salicylic acid related plant defense depends on sustained UPR activity. This is consistent with the observation that not only early but also late effectors require the UPR for efficient secretion and/or processing (Lo Presti et al., 2015b; Hampel et al., 2016; Pinter et al., 2019).

We provide a plasmid system for the community that allows the generation of constructs to be integrated into the genome in an efficient one step cloning procedure. Plasmids for conditional induction or repression of genes during biotrophic development in planta are cross compatible and harbor identical SfiI restriction sites for easy exchange of genes. Since the constructs can either be integrated in solopathogenic or in compatible haploid strains, future studies using combinations of conditionally expressed constructs will allow to address scientific questions (i.e. on phosphorylation/activity of a protein) related to fungal biotrophic growth in an efficient and sophisticated manner.

\subsection{Material and Methods}

\section{Strains and Growth Conditions}

Escherichia coli TOP10 strain was used for cloning purposes and amplification of plasmid DNA. U. maydis cells were grown at $28^{\circ} \mathrm{C}$ in YEPS light medium (Tsukuda et al., 1988), complete medium (CM) (Holliday, 1974) or yeast nitrogen base (YNB) medium (Mahlert et al., 2006; Freitag et al., 2011). Mating assays were performed das described before (Brachmann et al., 2001). ER-stress assays were carried out on YNB or CM media containing the indicated concentrations of DTT or TM (Sigma-Aldrich). Sensitivity towards Calcofluor White or Congo red was tested by drop-assay on YNB solid media containing the indicated concentration of the respective compound. Filamentous growth assays were carried out using potato-dextrose media supplemented with $1 \%$ charcoal (PD-CC) (Holliday, 1974). Strains used in this study are listed in Suppl. Table 4.1.

\section{DNA and RNA procedures}

Molecular methods followed described protocols (Sambrook et al., 1989). For gene deletions, a PCR-based approach was used (Kämper, 2004). Isolation of genomic DNA from $U$. maydis and transformation procedures were performed according to Schulz et al., 1990. Homologous integration was performed using linearized plasmid DNA or PCR amplified DNA. Integration was verified by Southern hybridization. Total RNA was 
extracted from exponentially growing cells in axenic culture using Trizol reagent (Invitrogen) according to the manufacturer's instructions. RNA integrity was checked by agarose-gel-electrophoresis. Residual DNA was removed from total RNA samples using the TURBO DNA-free ${ }^{\mathrm{TM}} \mathrm{Kit}$ (Ambion/Lifetechnologies). cDNA was synthesized using the iScript $^{\mathrm{TM}}$ cDNA Synthesis Kit. Primers used in this study are listed in Suppl. Table 4.2.

\section{Quantitative RT-PCR}

qRT-PCR analysis was performed as described before (Hampel et al., 2016). For all qRT-PCR experiments, three independent biological replicates and two technical replicates were analyzed using the MESA GREEN qPCR MasterMix plus for SYBR Assay with fluorescein (Eurogentech). qRT-PCR was performed using the CFX Connect Real-Time PCR Detection System and analyzed with the CFX Manager Maestro Software (BioRad).

\section{Plasmid construction}

For gene deletions, a PCR-based approach and the SfiI insertion cassette system was used (Brachmann et al., 2004; Kämper, 2004).

For construction of plasmids for conditional gene expression, $0.5-1 \mathrm{~kb}$ flanking regions of chosen genes (UMAG_03597, UMAG_12184, migl, mig2_1) were PCR amplified from genomic DNA, adding a SfiI restriction site to the 5' of the left border (LB) and a BamHI (for $U M A G_{-} 12184$, migl and mig2_l) or KpnI (for $U M A G_{-}$03597) restriction site to the 3 'end of the right border (RB). The gene of interest (GOI; GFP, mCherry, cibl or cibls) was also PCR amplified from genomic DNA or plasmid $\mathrm{P}_{c i b 1}: c i b 1^{\mathrm{s}}$ adding SfiI restriction sites to the 5' and 3'end. The resistance cassette was amplified from plasmid pUMa1442 adding a BamHI (for $U M A G_{-}$12184) or KpnI restriction site (for $U M A G \_03597$ ) to the 3 'end and a SfiI restriction site to the 5'end. The resulting DNA fragments were ligated to obtain $L B-G O I-H y g^{R}-R B$ or $L B-G O I-N a t^{R}-R B$. The final fragment was then integrated into the pCR2.1 TOPO vector (Invitrogen) or the pJet1.2 vector according to the manufacturer's instructions to generate plasmids pCR2.1 $\mathrm{P}_{U M A G_{-} 12184: C i b 1(\mathrm{NatR}),}$ pCR2.1

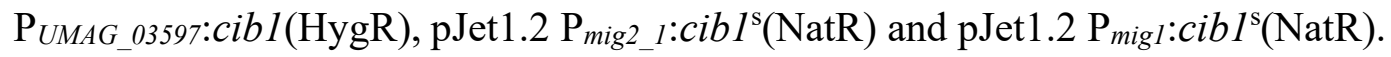

To construct the $\mathrm{P}_{\text {mig2_ }}$ : $:$ cibl $1^{\mathrm{s}}$ construct for $i p$ locus integration, the vectors Mig2_1:clp1 and pRU11-cib1s were cut with NdeI and EcoRI. The resulting $2.0 \mathrm{~kb} \mathrm{cibl}$ s fragment of 
Chapter 4 | Tool for conditional gene expression

pRU11-cib1s (Heimel et al., 2013) and the $5.2 \mathrm{~kb}$ backbone of Mig2_1:clp1 were ligated to obtain plasmid $\mathrm{P}_{\text {mig2_l }}$ : $c i b 1^{\mathrm{s}}$.

\section{Plant Infections}

The haploid, solopathogenic strain SG200 and its derivatives or FB1 and FB2 and their respective derivatives were grown to an OD600 of 0.6-0.8 in YEPS light medium, adjusted to an OD600 of 1.0 in water and mixed 1:1 with a compatible mating partner. $0.5 \mathrm{~mL}$ of the resulting suspension was used to inoculate 8-day-old maize seedlings of the variety Early Golden Bantam. Plants were grown in a CLF Plant Climatics GroBank with a $14 \mathrm{~h}$ $\left(28^{\circ} \mathrm{C}\right)$ day and $10 \mathrm{~h}\left(22^{\circ} \mathrm{C}\right)$ night cycle. Symptoms were scored according to disease rating criteria reported by Kämper et al., 2006. Three independent clones were used for each plant infection experiment and the average scores for each symptom are shown in the respective diagrams. Photos from infected leaves were taken und represent the most common infection symptom for the respective mutant.

\section{Chlorazole Black E staining and microscopy}

Infected leaf tissue was harvested at 2, 4 and 6 dpi and kept in 100\% ethanol until further processing. Chlorazole Black E staining was performed as described in Brachmann et al., 2001. Microscopic analysis was performed using an Axio Imager.M2 equipped with an AxioCam MRm camera (ZEISS). All images were processed using ImageJ.

\section{Quantification of $U$. maydis gene expression in planta and PR gene expression}

Infected leaf tissue was harvested at the indicated time points and samples of five infected maize seedlings were harvested and frozen in liquid nitrogen according to Lanver et al., 2018. Total RNA was extracted using Trizol reagent (Invitrogen) and used for qRT-PCR analysis as described above. For expression analysis of $U$. maydis genes, $e I F 2 b$ expression levels were used for normalization. Expression of $P R 1, P R 3$ and $P R 5$ from Zea mays were determined and normalized to GAPDH expression levels. 
Chapter 4 | Tool for conditional gene expression

\section{Statistical Analysis}

Statistical significance was calculated using Student's $t$ test. Results were considered significant if the $\mathrm{P}$ value was $<0.05$.

\section{Accession numbers}

Sequence data from this article can be found in the National Center for Biotechnology Information database under the following accession numbers:

UMAG_12184, XP_011388913.1; UMAG_03597, XP_011390022.1; cib1, UMAG_11782, XP_011390112.1; mig2_1, UMAG_06178, XP_011392548.1; mig1, UMAG_03223, XP_011389652.1; pit1, UMAG_01374, XP_011387263.1, pit2, UMAG_01375, XP_011387264.1; PR1 (Zm.15280.1), BM351351; PR3 (Zm.1085.1), BM339391; PR5 (Zm.6659.1), BM075306; GAPDH (NM001111943) 
Chapter 4 | Tool for conditional gene expression

\section{Supporting Information}

\section{s1}
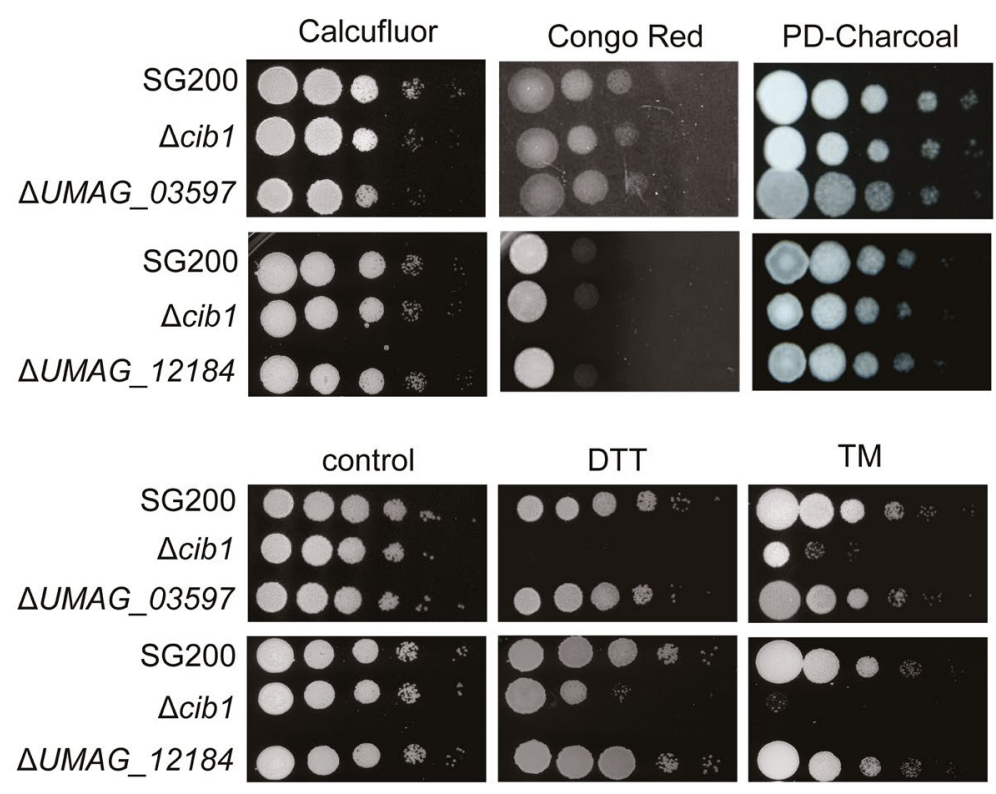

Suppl. Figure 4.1 $\triangle U M A G_{-} 12184$ and $\triangle U M A G_{-} 03597$ strains do not show increased sensitivity towards cell wall- or ER-stress. Cell wall-, ER-stress assay and test for filamentous growth of strains SG200,

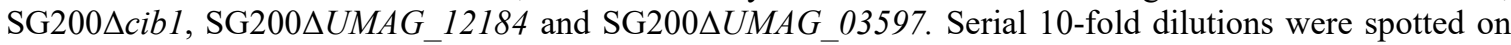
YNBG solid media supplemented with Congo Red $(100 \mu \mathrm{g} / \mathrm{mL})$ or Calcofluor White $(50 \mu \mathrm{M})$ to induce cell wall stress, and on YNBG solid media supplemented with TM $(2.0 \mu \mathrm{g} / \mathrm{mL})$ or DTT (1 mM) to induce ER-stress. Cells were spotted on PD-CC solid media to induce filamentous growth. Pictures were taken after 48 hours of incubation at $28^{\circ} \mathrm{C}$.

Suppl. Table 4.1 Strains used in this study

\begin{tabular}{|c|c|c|}
\hline Strain & Relevant Genotype & Reference \\
\hline FB1 & al bl & Banuett and Herskowitz, 1989 \\
\hline FB2 & $a 2 b 2$ & Banuett and Herskowitz, 1989 \\
\hline SG200 & al mfa2 bE1bW2 & Kämper et al., 2006 \\
\hline FB1 $1 \Delta c i b 1$ & al bl $\Delta c i b 1$ & Heimel et al., 2010 \\
\hline FB2 $\Delta c i b 1$ & $a 2 b 2 \Delta c i b 1$ & Heimel et al., 2010 \\
\hline UMH168 & almfa2 bE1bW2 $\Delta p i t 1 / 2 i p^{r} \mathrm{P}_{p i t 1 / 2}: p i t 1 / 2 i p^{s}$ & Hampel, 2016 \\
\hline UMH172 & almfa2 bE1bW2 $\Delta$ pit1/2:pit1/2 $\left(\mathrm{Nat}^{\mathrm{R}}\right)$ & Hampel, 2016 \\
\hline UMH247 & almfa2 bE1bW2 $\Delta$ pit1/2::pit1/2 $\left(\mathrm{Hyg}^{\mathrm{R}}\right)$ & Hampel, 2016 \\
\hline UMH268 & almfa2 bE1bW2 $\Delta$ pit1/2::pit1/2 (-Hyg $\left.{ }^{\mathrm{R}}\right)$ & Hampel 2016 \\
\hline SG200_umag_12184 & almfa2 bE1bW2 $\triangle U M A G \_12184$ & This study \\
\hline SG2004umag_03597 & almfa2 bE1bW2 $\triangle U M A G \_03597$ & This study \\
\hline ULS62 & al mfa2 bE1bW2 mip $\mathrm{P}_{\text {mig2_l }}: c i b 1^{s} i p^{s}$ & This study \\
\hline ULS251 & al b1 $\Delta c i b 1 \Delta m i g 2 \_1: \because c i b 1^{s}\left(+N a t^{R}\right)$ & This study \\
\hline ULS264 & al b1 $\Delta c i b 1 \Delta m i g 1:: c i b 1^{s}\left(-N a t^{R}\right)$ & This study \\
\hline
\end{tabular}




\begin{tabular}{lll} 
Strain & Relevant Genotype & Reference \\
\hline ULS270 & $a 1 b 1 \Delta c i b 1 \Delta m i g 2 \_1:: c i b 1^{s}\left(-N a t^{R}\right)$ & This study \\
ULS282 & $a 2 b 2 \Delta c i b 1 \Delta U M A G_{-} 12184: \because c i b 1$ & This study \\
ULS299 & $a 2 b 2 \Delta c i b 1 \Delta U M A G_{-} 03597:: c i b 1$ & This study \\
ULS352 & $a 1$ mfa2 bE1bW2 $p^{r} P_{\text {mig2_l }}: c i b 1^{s} i p^{s}$ & This study
\end{tabular}

Suppl. Table 4.2 Primers used in this study

\begin{tabular}{|c|c|c|c|}
\hline Purpose & Name & Sequence & Reference \\
\hline \multirow[t]{13}{*}{ qRT-PCR } & RT_cib1_rev & CATCGACGTTGTTTCCGGCCT & $\begin{array}{l}\text { Heimel et al., } \\
\text { 2010a }\end{array}$ \\
\hline & RT_cib1_spliced_f & GCCTCCCTGCAGCGGATGC & $\begin{array}{l}\text { Heimel et al., } \\
\text { 2010a }\end{array}$ \\
\hline & RT_eIF2b_f & ATCCCGAACAGCCCAAAC & $\begin{array}{l}\text { Heimel et al., } \\
\text { 2010a }\end{array}$ \\
\hline & RT_eIF2b_r & ATCGTCAACCGCAACCAC & $\begin{array}{l}\text { Heimel et al., } \\
\text { 2010a }\end{array}$ \\
\hline & RT_pr1_f & ACTACGTGGACCCGCACAAC & $\begin{array}{l}\text { Doehlemann et } \\
\text { al., } 2008\end{array}$ \\
\hline & RT_pr1_r & CGGAGTGGATCAGCTTGCAGTC & $\begin{array}{l}\text { (Doehlemann et } \\
\text { al., 2008) }\end{array}$ \\
\hline & RT_pr3_f & GAACAACTACAGCAGCCAGGTG & $\begin{array}{l}\text { Doehlemann et } \\
\text { al., } 2008\end{array}$ \\
\hline & RT_pr3_r & GAGACAATAGCTGACATGCGTC & $\begin{array}{l}\text { Doehlemann et } \\
\text { al., } 2008\end{array}$ \\
\hline & RT_pr5_f & TATCGGCCGGAATAGGCTCTG & $\begin{array}{l}\text { Doehlemann et } \\
\text { al., } 2008\end{array}$ \\
\hline & RT_pr5_r & CGCGTACATACAAATGCGTGC & $\begin{array}{l}\text { Doehlemann et } \\
\text { al., } 2008\end{array}$ \\
\hline & Pit1-RT-fw & GCCCAGTCCCAGTCTATC & $\begin{array}{l}\text { Doehlemann et } \\
\text { al., 2008, } \\
\text { modified }\end{array}$ \\
\hline & Pit1-RT-rev & GAAGGGGAGCAGGAGATG & $\begin{array}{l}\text { Doehlemann et } \\
\text { al., 2011, } \\
\text { modified }\end{array}$ \\
\hline & Pit2-RT-fw & CAAGAATCCGCCTGCCAACA & $\begin{array}{l}\text { Doehlemann et } \\
\text { al., 2011, } \\
\text { modified }\end{array}$ \\
\hline
\end{tabular}


Chapter 4 | Tool for conditional gene expression

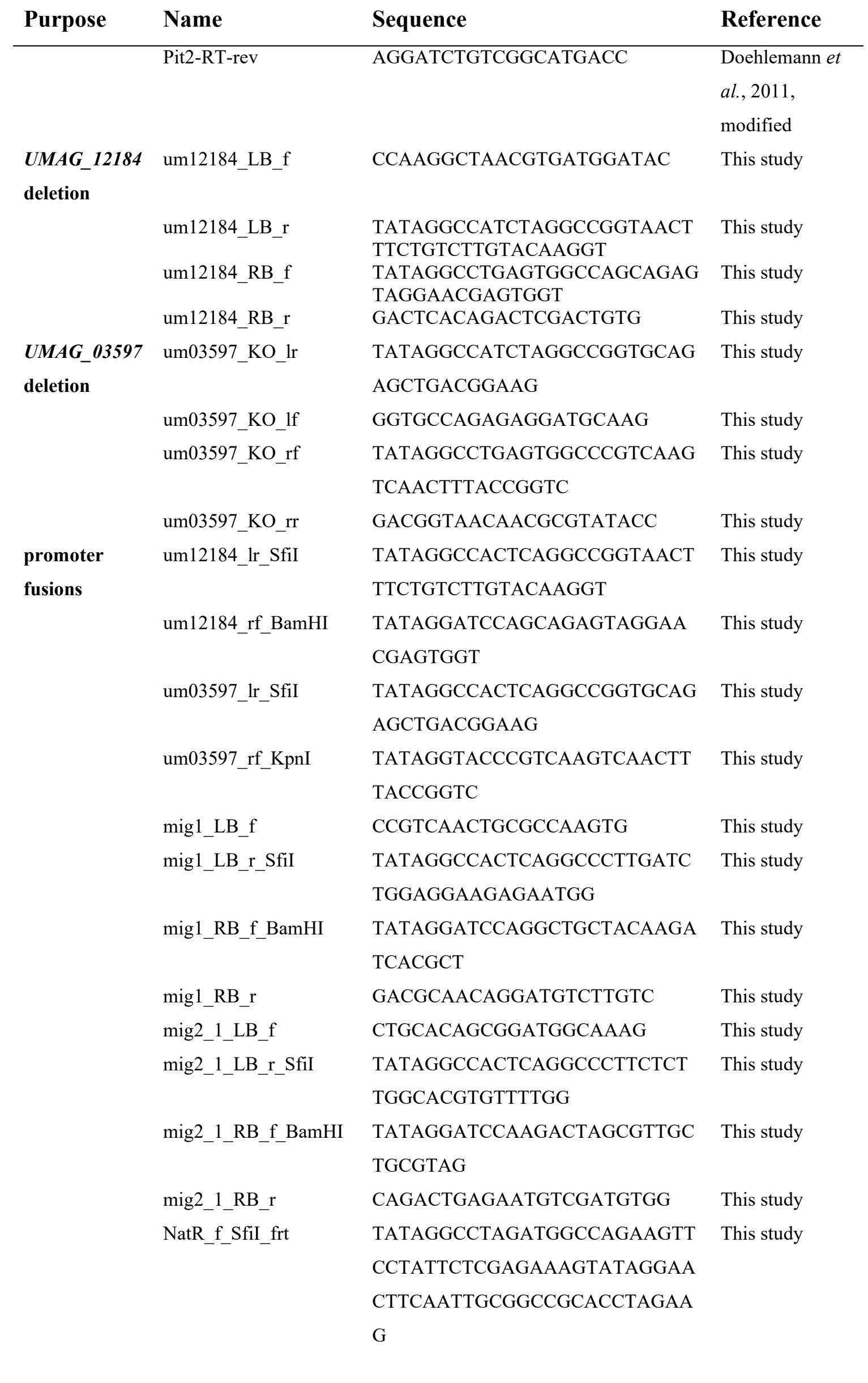


Chapter 4 | Tool for conditional gene expression

\begin{tabular}{llll} 
Purpose & Name & Sequence & Reference \\
\hline NatR_r_frt_BamHI & TATAGGATCCAGAAGTTCCTATAC & This study \\
& TTTCTCGAGAATAGGAACTTCGCG & \\
& GCCGCACTCCTACA & \\
Hyg_f & TATAGGCCTAGATGGCCAGAAGTT & This study \\
& C & \\
Hyg_r_KpnI & TATAGGTACCGAAGTTCCTATACT & This study \\
& TTCTAGAGAATAG & \\
cib1s_SfiI_f & TATAGGCCTGAGTGGCCATGACTA & This study \\
& GCACCACCACGTCA & \\
& TATAGGCCATCTAGGCCTCAAGCG & This study \\
& ACGATTGAGGCCAT &
\end{tabular}

\section{Acknowledgements}

We thank Daniel Lanver and Regine Kahmann for sharing expression data prior to publication. All authors acknowledge funding by the Deutsche Forschungsgemeinschaft (DFG), and the Natural Sciences Engingeering Council of Canada (NSERC) through the International Research Training Group 2172 PRoTECT and a Discovery Grant (to JWK).

\section{References}

Bakkeren, G., Kämper, J., and Schirawski, J. (2008) Sex in smut fungi: Structure, function and evolution of mating-type complexes. Fungal Genet Biol 45: 15-21.

Banks, G.R., Shelton, P.A., Kanuga, N., Holden, D.W., and Spanos, A. (1993) The Ustilago maydis narl gene encoding nitrate reductase activity: sequence and transcriptional regulation. Gene 131: 69-78.

Banuett, F. (1995) Genetics of Ustilago maydis, a Fungal Pathogen That Induces Tumors in Maize. Ann Rev Genet 29: 179-208.

Banuett, F., and Herskowitz, I. (1989) Different a alleles of Ustilago maydis are necessary for maintenance of filamentous growth but not for meiosis. Proc Natl Acad Sci 86: 58785882.

Barton, M.C., and Crowe, A.J. (2001) Chromatin alteration, transcription and replication: 
Chapter 4 | Tool for conditional gene expression

What's the opening line to the story? Oncogene 20: 3094-3099.

Basse, C.W., Kolb, S., and Kahmann, R. (2002) A maize-specifically expressed gene cluster in Ustilago maydis. Mol M 43: 75-93.

Basse, C.W., Stumpferl, S., and Kahmann, R. (2000) Characterization of a Ustilago maydis Gene Specifically Induced during the Biotrophic Phase: Evidence for Negative as Well as Positive Regulation. Mol Cell Proteomics 20: 329-339.

Bölker, M. (2001) Ustilago maydis - A valuable model system for the study of fungal dimorphism and virulence. Microbiology 1435: 24-1395.

Bölker, M., Urban, M., and Kahmann, R. (1992) The a mating type locus of $U$. maydis specifies cell signaling components. Cell 68: 441-450.

Bottin, A., Kämper, J., and Kahmann, R. (2002) Isolation of a carbon source-regulated gene from Ustilago maydis. MGG Mol Gen Genet 253: 342.

Brachmann, A. (2001) Die frühe Infektionsphase von Ustilago maydis: Genregulation durch das bW/bE-Heterodimer. München, Ludwig-Maximilians-Universität. Doktorarbeit.

Brachmann, A., König, J., Julius, C., and Feldbrügge, M. (2004) A reverse genetic approach for generating gene replacement mutants in Ustilago maydis. Mol Genet Genomics 272: 216-226.

Brachmann, A., Weinzierl, G., Kämper, J., and Kahmann, R. (2001) Identification of genes in the bW/bE regulatory cascade in Ustilago maydis. Mol Microbiol 42: 1047-1063.

Cox, J.S., and Walter, P. (1996) A Novel Mechanism for Regulating Activity of a Transcription Factor That Controls the Unfolded Protein Response. Cell 87: 391-404.

Dean, R., Kan, J.A.L. Van, Pretorius, Z.A., Hammond-Kosack, K.E., Pietro, A. Di, Spanu, P.D., et al. (2012) The Top 10 fungal pathogens in molecular plant pathology. Mol Plant Pathol 13: 414-30.

Doehlemann, G., Reissmann, S., Aßmann, D., Fleckenstein, M., and Kahmann, R. (2011) Two linked genes encoding a secreted effector and a membrane protein are essential for Ustilago maydis-induced tumour formation. Mol Microbiol 81: 751-766.

Feldbrügge, M., Kämper, J., Steinberg, G., and Kahmann, R. (2004) Regulation of mating and pathogenic development in Ustilago maydis. Curr Opin Microbiol 7: 666-672. 
Freitag, J., Lanver, D., Böhmer, C., Schink, K.O., Bölker, M., and Sandrock, B. (2011) Septation of infectious hyphae is critical for appressoria formation and virulence in the smut fungus Ustilago maydis. PLoS Pathog 7: 1-15.

Glazebrook, J. (2005) Contrasting Mechanisms of Defense Against Biotrophic and Necrotrophic Pathogens. Annu Rev Phytopathol 43: 205-227.

Hampel, M., Jakobi, M., Schmitz, L., Meyer, U., Finkernagel, F., Doehlemann, G., and Heimel, K. (2016) Unfolded Protein Response (UPR) Regulator Cib1 Controls Expression of Genes Encoding Secreted Virulence Factors in Ustilago maydis. PLoS One 11: 1-16.

Hartmann, H.A., Kahmann, R., and Bölker, M. (1996) The pheromone response factor coordinates filamentous growth and pathogenicity in Ustilago maydis. EMBOJ 15: 16321641.

Heimel, K., Freitag, J., Hampel, M., Ast, J., Bolker, M., and Kämper, J. (2013) Crosstalk between the Unfolded Protein Response and Pathways That Regulate Pathogenic Development in Ustilago maydis. Plant Cell 25: 4262-4277.

Heimel, K., Scherer, M., Schuler, D., Kämper, J. (2010a) The Ustilago maydis Clp1 Protein Orchestrates Pheromone and $b$-Dependent Signaling Pathways to Coordinate the Cell Cycle and Pathogenic Development. Plant Cell 22: 2908-2922.

Heimel, K., Scherer, M., Vranes, M., Wahl, R., Pothiratana, C., Schuler, D., et al. (2010b) The transcription factor Rbfl is the master regulator for b-mating type controlled pathogenic development in Ustilago maydis. PLoS Pathog 6: 17-18.

Holliday, R. (1974) GENETICS Volume 1 Bacteria, Bacteriophages, and Fungi.

Kahmann, R., and Kämper, J. (2004) Ustilago maydis: How its biology relates to pathogenic development. New Phytol 164: 31-42.

Kämper, J. (2004) A PCR-based system for highly efficient generation of gene replacement mutants in Ustilago maydis. Mol Genet Genomics 271: 103-110.

Kämper, J., Kahmann, R., Bölker, M., Ma, L.-J., Brefort, T., Saville, B.J., et al. (2006) Insights from the genome of the biotrophic fungal plant pathogen Ustilago maydis. Nature 444: $97-101$.

Kämper, J., Reichmann, M., Romeis, T., Bölker, M., and Kahmann, R. (1995) Multiallelic 
Chapter 4 | Tool for conditional gene expression

recognition: Nonself-dependent dimerization of the $\mathrm{bE}$ and $\mathrm{bW}$ homeodomain proteins in Ustilago maydis. Cell 81: 73-83.

Kawahara, T., Yanagi, H., Yura, T., and Mori, K. (1998) Unconventional Splicing of HAC1/ERN4 mRNA Required for the Unfolded Protein Reponse. Biochemistry 273: 18021807.

Keon, J.P.R., White, G.A., and Hargreaves, J.A. (1991) Isolation, characterization and sequence of a gene conferring resistance to the systemic fungicide carboxin from the maize smut pathogen, Ustilago maydis. Curr Genet 19: 475-481.

Khrunyk, Y., Münch, K., Schipper, K., Lupas, A.N., and Kahmann, R. (2010) The use of FLP-mediated recombination for the functional analysis of an effector gene family in the biotrophic smut fungus Ustilago maydis. New Phytol 957-968.

Kluge, J., Terfehr, D., and Kück, U. (2018) Inducible promoters and functional genomic approaches for the genetic engineering of filamentous fungi. Appl Microbiol Biotechnol 102: 6357-6372.

Lanver, D., Müller, A.N., Happel, P., Schweizer, G., Haas, F.B., Franitza, M., et al. (2018) The Biotrophic Development of Ustilago maydis Studied by RNA-Seq Analysis. Plant Cell 30: $300-323$.

Lee, J., Son, H., Lee, S., Park, A.R., and Lee, Y.W. (2010) Development of a Conditional Gene Expression System Using a Zearalenone-Inducible Promoter for the Ascomycete Fungus Gibberella zeae. Appl Environ Microbiol 76: 3089-3096.

Mahlert, M., Leveleki, L., Hlubek, A., Sandrock, B., and Bölker, M. (2006) Rac1 and Cdc42 regulate hyphal growth and cytokinesis in the dimorphic fungus Ustilago maydis. Mol Microbiol 59: 567-578.

Pachlinger, R., Mitterbauer, R., Adam, G., and Strauss, J. (2005) Metabolically Independent and Accurately Adjustable Aspergillus sp. Expression System. Appl Environ Microbiol 71: 672-678.

Pinter, N., Hach, C.A., Hampel, M., Rekhter, D., Zienkiewicz, K., Feussner, I., et al. (2019) Signal peptide peptidase activity connects the unfolded protein response to plant defense suppression by Ustilago maydis. PLOS Pathog 15: 1-40.

Presti, L., Díaz, L., Turrà, D., Di Pietro, A., Hampel, M., Heimel, K., and Kahmann, R. 
(2015) A conserved co-chaperone is required for virulence in fungal plant pathogens. New Phytol 208: 1-14.

Presti, L., Lanver, D., Schweizer, G., Tanaka, S., Liang, L., Tollot, M., et al. (2015) Fungal Effectors and Plant Susceptibility. Annu Rev Plant Biol 66: 513-545.

Reichmann, M., Jamnischek, A., Weinzierl, G., Ladendorf, O., Huber, S., Kahmann, R., and Kämper, J. (2002) The histone deacetylase Hda1 from Ustilago maydis is essential for teliospore development. Mol Microbiol 46: 1169-1182.

Rüegsegger, U., Leber, J.H., and Walter, P. (2001) Block of HAC1 mRNA translation by long-range base pairing is released by cytoplasmic splicing upon induction of the unfolded protein response. Cell 107: 103-114.

Salinas, F., Rojas, V., Delgado, V., López, J., Agosin, E., and Larrondo, L.F. (2018) Fungal Light-Oxygen-Voltage Domains for Optogenetic Control of Gene Expression and Flocculation in Yeast. MBio 9: 1-14.

Sambrook, J., Frisch, E.F., and Maniatis, T. (1989) Molecular Cloning: A Laboratory Manual.

Schulz, B., Banuett, F., Dahl, M., Schlesinger, R., Schäfer, W., Martin, T., et al. (1990) The $b$ alleles of $U$. maydis, whose combinations program pathogenic development, code for polypeptides containing a homeodomain-related motif. Cell 60: 295-306.

Schuster, M., Schweizer, G., Reissmann, S., and Kahmann, R. (2016) Genome editing in Ustilago maydis using the CRISPR-Cas system. Fungal Genet Biol 89: 3-9.

Soyer, J.L., Ghalid, M. El, Glaser, N., Ollivier, B., Linglin, J., Grandaubert, J., et al. (2014) Epigenetic Control of Effector Gene Expression in the Plant Pathogenic Fungus Leptosphaeria maculans. PLoS Genet 10: 1-19.

Spellig, T., Bottin, A., and Kahmann, R. (1996) Green fluorescent protein (GFP) as a new vital marker in the phytopathogenic fungus Ustilago maydis. Mol Gen Genet 252: 503509.

Terfrüchte, M., Joehnk, B., Fajardo-Somera, R., Braus, G., Riquelme, M., Schipper, K., and Feldbrügge, M. (2014) Establishing a versatile Golden Gate cloning system for genetic engineering in fungi. Fungal Genet Biol 62: 1-10. 
Chapter 4 | Tool for conditional gene expression

Treutlein, M. (2007) Funktionelle Charakterisierung des Rum1-Proteins aus dem phytopathogenen Pilz Ustilago maydis. PhD Thesis.

Tsukuda, T., Carleton, S., Fotheringham, S., and Holloman, W.K. (1988) Isolation and characterization of an autonomously replicating sequence from Ustilago maydis. Mol Cell Biol 8: 3703-3709.

Wahl, R., Zahiri, A., and Kämper, J. (2010) The Ustilago maydis b mating type locus controls hyphal proliferation and expression of secreted virulence factors in planta. Mol Microbiol 75: 208-220.

Wittschieben, B., Fellows, J., Du, W., Stillman, D.J., and Svejstrup, J.Q. (2000) Overlapping roles for the histone acetyltransferase activities of SAGA and Elongator in vivo. EMBO J 19: 3060-3068.

Wittschieben, B., Otero, G., Bizemont, T. De, Fellows, J., Erdjument-Bromage, H., Ohba, R., et al. (1999) A novel histone acetyltransferase is an integral subunit of elongating RNA polymerase II holoenzyme. Mol Cell 4: 123-128.

Yoshida, H., Oku, M., Suzuki, M., and Mori, K. (2006) pXBP1(U) encoded in XBP1 premRNA negatively regulates unfolded protein response activator pXBP1(S) in mammalian ER stress response. J Cell Biol 172: 565-575.

Zarnack, K., Eichhorn, H., Kahmann, R., and Feldbrügge, M. (2008) Pheromone-regulated target genes respond differentially to MAPK phosphorylation of transcription factor Prf1. Mol Microbiol 69: 1041-1053. 


\section{Chapter 5 | Discussion}

This study provides new insights into the crosstalk between the UPR and the mating type signaling cascades and addressed the role of a functional UPR during biotrophic development of $U$. maydis in planta. Previous studies demonstrated an intricate connection between the UPR and the $b$-dependent regulatory network that is established via the physical interaction between the central UPR regulator Cib1 and Clp1, the decisive factor for fungal proliferation in planta. Here, we identified that crosstalk between these signaling-cascades is realized on a second level. An active UPR pathway inhibits the mating-type regulated transcriptional response and morphological transitions prior to plant infection. This effect depends on the dual specificity phosphatase (DSP) Rok1, which dephosphorylates the MAPK Kpp2, reducing Kpp2 activity and likely phosphorylation-dependent activation of the pheromone response factor (Prfl). In this manner, active UPR signaling inhibits expression of the $a$ - and $b$-mating type genes and ultimately suppresses pheromone-induced conjugation tube formation and b-dependent filamentous growth. We postulate that this interaction constitutes a regulatory feedback mechanism to fine-tune the mating type activity in planta to maintain biotrophy. By using a novel approach for conditional gene expression, we further show that UPR is not only essential to establish a biotrophic interaction after plant penetration, but is also important for the continuous suppression of plant defense responses at later stages of pathogenic development. Our study suggests that $U$. maydis utilizes robust control mechanisms to prevent deleterious effects from UPR hyperactivation, most likely established via negative feedback regulation of the Cib1 protein function. 


\subsection{UPR antagonizes MAPK dependent signaling}

\subsubsection{Active UPR signaling interferes with $a$ - and $b$-mating type dependent gene expression and b-dependent filament formation}

Fusion of two compatible haploid sporidia is regulated by the $a$-mating type locus, while all subsequent steps of pathogenic development are under control of the $b$-mating type locus. The $\mathrm{bE} / \mathrm{bW}$ heterodimer encoded by the $b$-mating-type locus facilitates maintenance of the G2 cell cycle arrest, filamentous growth, formation of appressoria and penetration of the plant surface (Bölker et al., 1995; Brachmann et al., 2001; Wahl et al., 2010b). However, fungal proliferation in planta requires modulation of the $b$-dependent pathway by the Clp1 protein. Clp1 is stabilized via the Cib1/Clp1 interaction and specifically accumulates after successful plant penetration suppressing the mating type dependent signaling pathways to release the cell cycle block and induce fungal proliferation (Heimel et al., 2010a; Heimel et al., 2013). Hence, the plant-specific activation of the UPR serves as a trigger for biotrophic growth. Consistently, Clp1 and Cib1 deletion strains are arrested in pathogenic development rapidly after plant penetration (Heimel et al., 2010a), demonstrating that both proteins are essential for pathogenic development.

Conversely, premature activation of the UPR inhibits b-dependent filament formation and expression of $b E, b W$ and $r b f 1$ is dose-dependently reduced (Heimel et al., 2013). Expression of the Rbf1-target genes bizl, $h d p 1$ and $h d p 2$ is also suppressed by the UPR, suggesting extensive inhibitory effects of the UPR on the transcription factor network. Rbf1 is required and sufficient to induce filamentous growth and controls expression of more than $90 \%$ of the $b$-dependently regulated genes (Heimel et al., 2010b). The transcription factors bizl, hdpl and $h d p 2$ have distinct functions during filamentous growth and penetration of the plant surface. The $\mathrm{C} 2 \mathrm{H} 2$ zinc finger transcription factor $\mathrm{Biz} 1$ is required for the G2 cell cycle arrest and induction of appressoria formation to penetrate the plant surface (Flor-Parra et al., 2006). Deletion of $h d p 1$ or $h d p 2$ results in impaired filament formation and the loss of pathogenicity, respectively (Heimel et al., 2010b). Thus, reduced filament formation likely depends on the inhibition of $r b f 1$ and of the Rbfl-regulated transcription factors $b i z l, h d p l$ and $h d p 2$.

Interestingly, inhibition of filament formation was also observed in strains overexpressing $b E$ and $b W$ (Heimel et al., 2013). Thus, UPR-dependent inhibition of filamentous growth 
is achieved by at least two distinct mechanisms, presumably involving factors operating upstream and downstream of $\mathrm{bE} / \mathrm{bW}$. Expression of $b E$ and $b W$ is controlled by the transcription factor Prf1. Consistently, transcription of prfl and the Prf1-regulated genes $m f a l$ and pral (encoding the pheromone/pheromone receptor, respectively) was inhibited by an active UPR. prfl gene expression is regulated by at least four different factors. In addition to autoregulation of Prfl and an unknown, Crk1-dependent factor integrating environmental cues, the transcription factors Rop1 and Hap2 are required for prf1 expression under axenic conditions (Brefort et al., 2005; Mendoza-Mendoza et al., 2009b). Interestingly, expression of both genes, although less pronounced for hap2, was significantly suppressed by an active UPR. Since the upstream MAPK Kpp2 regulates Hap2 activity via phosphorylation and is likely involved in the transcriptional regulation of ropl (Brefort et al., 2005; Mendoza-Mendoza et al., 2009b), reduced expression of both genes might be an indirect effect of Kpp2 inhibition. In conjunction with the observation that genes whose transcription depend on phosphorylation of MAPK sites in Prf1 (Zarnack et al., 2008) showed differential expression, phosphorylation and thereby activity of Prf1 is presumably reduced by the UPR.

\subsubsection{UPR-dependent dephosphorylation of the MAPK Kpp2 represses the morphological and transcriptional response to a pheromone stimulus}

The haploid U. maydis wild type strain FB1 was used to analyze UPR-dependent effects that act specifically on the pheromone-induced signaling pathways. Pheromone treatment activates cAMP- and a MAPK-signaling cascades that converge on Prfl through site-specific phosphorylation (Krüger et al., 1998; Hartmann et al., 1999; Müller et al., 1999). Phosphorylation of Prf1 by the cAMP-dependent PKA Adr1 triggers $a$-mating type gene expression, while conjugation tube formation depends on the MAPK Kpp2 in a Prf1-independent process (Kaffarnik et al., 2003; Müller et al., 2003). Sensing of a compatible pheromone increases/induces both, a-gene expression and formation of conjugation tubes (Urban et al., 1996). By contrast, active UPR signaling suppressed the transcriptional and the morphological response to pheromone. In the human pathogen Cryptococcus neoformans, sexual differentiation is regulated by the UPR via the kinase/endoribonuclease Ire1, but independent from Hxl1 (HACl and XBP1-Like gene 1) 
(Cheon et al., 2011). In contrast to U. maydis, IRE1 deletion strongly induces expression of the $M F \alpha 1$ pheromone gene, but inhibits pheromone-induced formation of conjugation structures and cell-cell fusion (Jung et al., 2016).

Expression analysis revealed that an active UPR only has minor effects on cAMP-induced transcription of $m f a l$ and pral, indicating that the inhibitory effect of the UPR acts mainly on the MAPK-signaling module. In S. cerevisiae, a MAPK module consisting of the MAPKKK Ste11, the MAPKK Ste7 and the MAPK Fus3 (Kpp2 ortholog) or Kss1 regulates mating and filamentous growth in response to a pheromone stimulus and nitrogen/carbon starvation (Liu et al., 1993; Zhou et al., 1993). Activation of the MAPK Fus3p induces the pheromone (mating) pathway, but inhibits the filamentation pathway, while activity of the MAPK Kss1p activates both pathways (Cook et al., 1997; Madhani et al., 1997). Interestingly, nitrogen starvation-induced pseudohyphal growth is suppressed by the UPR (Schröder et al., 2000). Further results show that Haclp interacts with the RPD3-SIN3 histone deacetylase complex to suppress expression of early meiotic genes, thereby repressing entry into meiosis and growth arrest (Schröder et al., 2004). However, the molecular mechanisms underlying UPR-dependent inhibition of pseudohyphal growth in yeast have not been fully resolved, yet.

In U. maydis, genetic activation of the pheromone-responsive MAPK module by induced expression of the constitutive active MAPKK Fuz7 $\left(f u z 7^{D D}\right)$ leads to increased expression of the $a$-mating type genes and formation of conjugation tube-like structures (Müller et al., 2003). In strains with an active UPR, the transcriptional but not the morphological response to genetic activation of the pheromone pathway was inhibited. Consistently, it has been reported that the morphological and transcriptional pheromone response bifurcate downstream of Kpp2 (Müller et al., 2003). Induced expression $f u z 7^{D D}$ resulted in Kpp2 phosphorylation, however, Kpp2 phosphorylation was almost completely abolished in strains with an active UPR. Since phosphorylation is essential for catalytic activity of MAPKs (Cargnello and Roux, 2011), these results suggest that activity of Kpp2 is not required for conjugation tube formation. This is contrasting previous reports showing that strains expressing the non-phosphorylatable $\mathrm{Kpp} 2^{\mathrm{AEF}}$ or the kinase-dead $\mathrm{Kpp} 2^{\mathrm{K} 50 \mathrm{R}}$ mutant protein do not form conjugation tubes upon pheromone stimulation (Müller et al., 2003). However, genetic activation of the MAPK module via $f u z 7^{D D}$ might result in different signaling outputs based on the interference with feedback loops and signaling dynamics. Alternatively, Kpp2 might function independent of its phosphorylation status/catalytic 
activity. In S. cerevisiae, the non-phosphorylated MAPK Kss1 binds to and thereby represses the transcription factor Ste12. By contrast, signals inducing the filamentation pathway lead to phosphorylation of Kss1, thereby weakening the interaction with Ste12 which is in turn derepressed and promotes transcription of target genes required for filamentous growth (Cook et al., 1997; Bardwell et al., 2008).

Crosstalk between the UPR and MAPK signaling cascades appears not to be restricted to U. maydis, but to be common in fungi. In Aspergillus fumigatus and C. neoformans, deletion of the genes encoding Hacl or Irel homologs increased sensitivity towards cell wall stress (Richie et al., 2009; Cheon et al., 2011; Feng et al., 2011). In S. cerevisiae, $\Delta$ irel mutants have defects in cell wall integrity and upon cell wall stress, the UPR is induced via the CWI MAPK Mpk1p (Scrimale et al., 2009). However, in U. maydis, sensitivity towards cell wall stress-inducing agents was not increased in cibl deletion strains (Pinter et al., 2019), indicating that the UPR is not required for the cell wall stress response.

\subsubsection{UPR-dependent suppression of Kpp2 phosphorylation depends on the DSP Rok1}

Signal transduction in MAPK modules is promoted by scaffold proteins, gathering individual module components, bringing them in close proximity to each other, thereby fostering and stabilizing protein interactions. Hence, scaffolds can influence/control the activity of MAPK modules (Bardwell, 2006; Dard and Peter, 2006; Good et al., 2011). In S. cerevisiae, the scaffold protein STE5 is essential for the mating process by providing an interaction platform for kinases of the pheromone-dependent MAPK module (Elion, 1995). Moreover, scaffolds can induce conformational changes of kinases or recruit phosphatases to inactivate MAPKs (Willoughby et al., 2003; Mapes and Ota, 2004; Good et al., 2009). The scaffold protein Nbp2p binds the MAPK Hog1p, which is part of the osmoregulatory signal transduction cascade, and mediates Hog $1 \mathrm{p}$ dephosphorylation by recruiting the phosphatases Ptc1p and Pbs2p (Mapes and Ota, 2004). However, no such scaffold protein has been identified in $U$. maydis, yet. In addition, the interaction between Fuz $7^{\mathrm{DD}}$ and Kpp2 is not affected by UPR activity, suggesting that reduced Kpp2 phosphorylation is not connected to scaffold proteins or an altered interaction between MAPKK and MAPK. 
Another level of regulation of kinase activity is mediated by phosphatases that serve as suppressors of kinase-dependent signaling by dephosphorylation of their target kinases (González-Rubio et al., 2019). In U. maydis, Kpp2 is dephosphorylated by the DSP Rok1. Deletion of rok1 leads to increased and overexpression to strongly reduced Kpp2 phosphorylation, respectively. This correlates with enhanced or reduced activity of the pheromone pathway, as evidenced by the regulation of target genes, including $b E$ and $b W$ (Di Stasio et al., 2009). Importantly, Rok1 activity appears to be increased by the UPR, leading to Kpp2 dephosphorylation and inactivation of the MAPK signaling module.

Mitogen activated kinase phosphatases (MKP) are regulated on multiple levels to ensure tight control of the corresponding signaling pathway. This includes transcriptional as well as post-translational regulation. Commonly, expression of MKPs is transcriptionally induced upon activation of the corresponding signaling cascade placing them in a negative feedback loop. Consistently, expression of rokl and the rokl ortholog in S. cerevisiae, MSG5, is upregulated upon pheromone stimulation (Doi et al., 1994; Di Stasio et al., 2009). Hence, elevated Rok1 protein levels are likely linked to the increased rok1 expression but might as well be connected to altered protein stability. Since UPR activity neither affected transcription nor protein levels of Rok1, it appears likely that the UPR regulates Rok1 activity.

Post-translational modifications of Rok1-like MKPs include phosphorylation and ubiquitination and can alter localization, stability and activity of the phosphatase (Lawan et al., 2013). Two potential Rok1 phosphorylation sites have been predicted by in silico analysis. However, their role in regulation of Rok1 is unknown (Di Stasio, 2009b), and although phosphorylation of Rok1 could be confirmed by LC-MS analysis, no effects of UPR activity on Rok1 phosphorylation were detectable (data not shown). In the hemibiotrophic blast fungus Magnapothe oryzae (Pyricularia oryzae), the tyrosine-protein phosphatase Pmp1 is phosphorylated at a conserved serine residue and subsequently dephosphorylates the MAPKs Pmk1 (Fus3/Kpp2 ortholog) and Mps1 (Slt2 ortholog) (Wang et al., 2017). In yeast, phosphorylation of the MKP Msg5p by the target kinases Fus3 and Slt2 results in increased Msg5p activity (Doi et al., 1994; Flández et al., 2004). This establishes a negative feedback loop in which Msg5p is phosphorylated by Slt2 upon cell wall stress and in turn dephosphorylates Slt2. In mammals, phosphorylation of MKP-1 on serine 359 and 364 by ERK1/2 enhances its stability (Brondello et al., 1999), whereas phosphorylation of serine 296 mediates ubiquitination and proteasomal degradation of 
MKP-1 (Lin et al., 2003; Lin and Yang, 2006). Moreover, acetylation of MKP-1 in macrophages promotes the interaction and dephosphorylation of the MAPK p38 (Cao et al., 2008).

While Kpp2 is localized in the cytoplasm, it is translocated to the nucleus upon pheromone stimulation (Müller, 2003). Rok1 is presumably localized to the plasma membrane and septa and re-localizes to the ER upon pheromone stimulus (Di Stasio, 2009a). However, due to low rok1 expression levels, the subcellular localization of Rok1 in strains with active UPR could not be addressed, yet. In S. cerevisiae, Msg5 is evenly distributed in the cytoplasm and the nucleus independent of pheromone stimulus, while Fus $3 p$ is predominantly found in the nucleus under inducing conditions. However, during recovery from the pheromone stimulus, Fus3p is dephosphorylated by Msg5p resulting in a decrease of nuclear Fus3p (Blackwell et al., 2003; Blackwell et al., 2007). Localization studies of Kpp2 and Rok1 in strains with and without active UPR could provide important insights into the underlying regulatory mechanisms.

Deletion of rok1 restored phosphorylation of Kpp2 upon UPR activity, demonstrating dephosphorylation of Kpp2 by Rok1 in response to UPR activation. However, expression of pheromone pathway genes was still suppressed, strongly suggesting that additional UPR-mediated mechanisms affect Kpp2 activity. In S. cerevisiae, Msg5p and the two tyrosine-specific phosphatases Ptp2 and Ptp3 coordinately dephosphorylate Fus3 (Zhan et al., 1997). Overexpression of PTP3 inactivates Fus3p, thereby reducing expression of pheromone-responsive genes and promoting recovery from cell cycle arrest. An orthologous gene to PTP3, UMAG_10534, has been identified in U. maydis. However, attempts to delete $U M A G_{-} 10534$ failed, indicating essential functions of the gene (Di Stasio, 2009b). Interestingly, ChIPseq data suggest that Cib1 directly binds to the promoter region of $U M A G_{-} 10534$ (Pinter et al., 2019). However, no effects on gene expression were observed upon UPR activation.

Alternatively, an additional layer of crosstalk between the UPR and the MAPK signaling cascade might exist that affects transcription or activity of $\mathrm{prfl}$. Hence, the regulators of prf1 expression, Rop1 and Hap2, or a mechanism acting via the MAPK Crk1 (Garrido and Pérez-Martín, 2003; Garrido et al., 2004; Brefort et al., 2005; Mendoza-Mendoza et al., $2009 \mathrm{~b}$ ), might account for reduced expression of $p r f 1$ and $a$-mating type genes under these conditions. It appears unlikely that the cAMP-dependent PKA pathway is involved since 
Chapter 5 | Discussion

transcriptional profiling did not reveal major alterations of regulatory genes or target gene expression by the UPR.

\subsubsection{The UPR and mating type-dependent signaling cascades interact on multiple levels}

Crosstalk between the UPR and the $a$-mating type pathway inhibits $a$ - and $b$-mating type gene expression and $b$-dependent filament formation. Surprisingly, constitutive expression of the $b E / b W$ heterodimer did neither restore expression of the $b$-dependent transcription factors nor filament formation in strains with active UPR. This suggests that the pheromone-dependent signaling cascade and the $b$-dependent transcription factor network are indeed regulated by two distinct, UPR-dependent mechanisms as previously hypothesized (Figure 5.1) (Heimel et al., 2013). The inhibition of bW and Rbfl by binding to the developmental regulator Clp1 (Scherer et al., 2006; Heimel et al., 2010a) could account for reduced expression of $r b f l$, Rbfl target genes, and inhibition of filamentous growth. UPR activation results in stabilization and accumulation of $\mathrm{Clp} 1$ and therefore is expected to enhance the inhibitory effects of Clp1 on bW and Rbfl (Heimel et al., 2010a; Heimel et al., 2013). In conclusion, deletion of rokl and clp1 would be expected to disrupt the UPR-dependent inhibition and restore filament formation and gene expression.

An alternative explanation for reduced $r b f l$ levels in strains constitutively expressing $b E / b W$ would be a post-translational modification of the $\mathrm{bE} / \mathrm{bW}$-complex, potentially caused by inhibition of the MAPK pathway and Prf1. In addition to the b-dependent regulation of gene expression, $r b f l$ is also induced by the pheromone pathway (Zarnack et $a l ., 2008)$. Thus, the UPR-dependent inhibition of $p r f l$ and the $a$-mating type genes might result in reduced $r b f 1$ expression independent of $\mathrm{bE} / \mathrm{bW}$. However, constitutive expression of $b E / b W$ under these conditions is expected to prevent negative feedback regulation.

\subsubsection{Role of the Kpp2-Rok1 interaction during pathogenic development in planta}

The UPR is specifically activated after penetration of the plant surface, while the MAPK Kpp2 is mainly active during the mating process, filamentous growth on the plant surface and appressoria formation (Müller et al., 1999). We observed that, although Kpp2 is constitutively expressed during plant infection (Lanver et al., 2018), it is dispensable for pathogenic development in planta. Thus, the relevance of the UPR-Kpp2 crosstalk via the phosphatase Rok1 during pathogenic development remains elusive. Interestingly, the 
related MAPK Kpp6 acts in the same MAPK module and is partially redundant to Kpp2 (Brachmann et al., 2003; Müller et al., 2003). Thus, Kpp6 might substitute Kpp2 during later stages of development. Kpp6 is also phosphorylated by the MAPK Fuz7 and controlled by the DSP Rok1 (Di Stasio et al., 2009). Yeast-2-Hybrid analysis confirmed a direct interaction with Prf1 (Mendoza-Mendoza et al., 2009a) and Rok1 (this study). Although Kpp6 is rbfl-dependently expressed, two PRE motifs in the promoter have been identified indicating additional regulation by Prf1 (Brachmann et al., 2003). Besides being partially redundant to Kpp2 during mating and development on the plant surface, Kpp6 is specifically required for appressorial function (Brachmann et al., 2003). Furthermore, Kpp6 contains a unique N-terminal extension, suggesting additional functions/regulation of Kpp6. Since U. maydis forms appressoria-like structures during cell-to-cell growth (Doehlemann et al., 2009), Kpp6 is potentially also required after plant penetration. The surface sensing protein Sho1 acts upstream of Kpp6 and regulates formation of appressoria together with Msb2. Sho1 interacts with the N-terminal extension of Kpp6 and thereby destabilizes Kpp6 (Lanver et al., 2010a). It was hypothesized that Sho1 and Msb2 have additional, Fuz7-dependent functions during biotrophic development in planta, which potentially involve fine-tuning of Kpp6 levels. However, the possibility that Kpp6 replaces Kpp2 during biotrophic growth in planta and a potential UPR-dependent regulation of Kpp6 has to be further elucidated.

Biotrophic pathogens like U. maydis rely on a living host to complete their life cycle. Hence, control mechanisms are required to ensure efficient host-colonization and to maintain the biotrophic interaction. It is conceivable that the UPR-mediated inhibition of the mating type signaling pathways after plant penetration provides a regulatory feedback control to balance the activity of the signaling cascades. In rokl deletion strains, phosphorylation of the MAPK Kpp2 and Kpp6 is strongly enhanced leading to increased $b$-gene expression and hypervirulence (Di Stasio et al., 2009). Regulation of Rok1 by UPR activity might constitute a mechanism to dampen the $a$ - and $b$-mating type signaling pathways and prevent hypervirulence. Moreover, effector gene expression is largely controlled by the b-heterodimer and $b$-dependent transcription factors, such as Rbf1, Hdp2 and Biz1 (Flor-Parra et al., 2006; Heimel et al., 2010b; Lanver et al., 2014; Lanver et al., 2018; Schmitz et al., 2018). Hence, inhibition of the signaling cascades by increased UPR activity would reduce effector gene expression and thereby reduce ER stress levels. 
Chapter 5 | Discussion
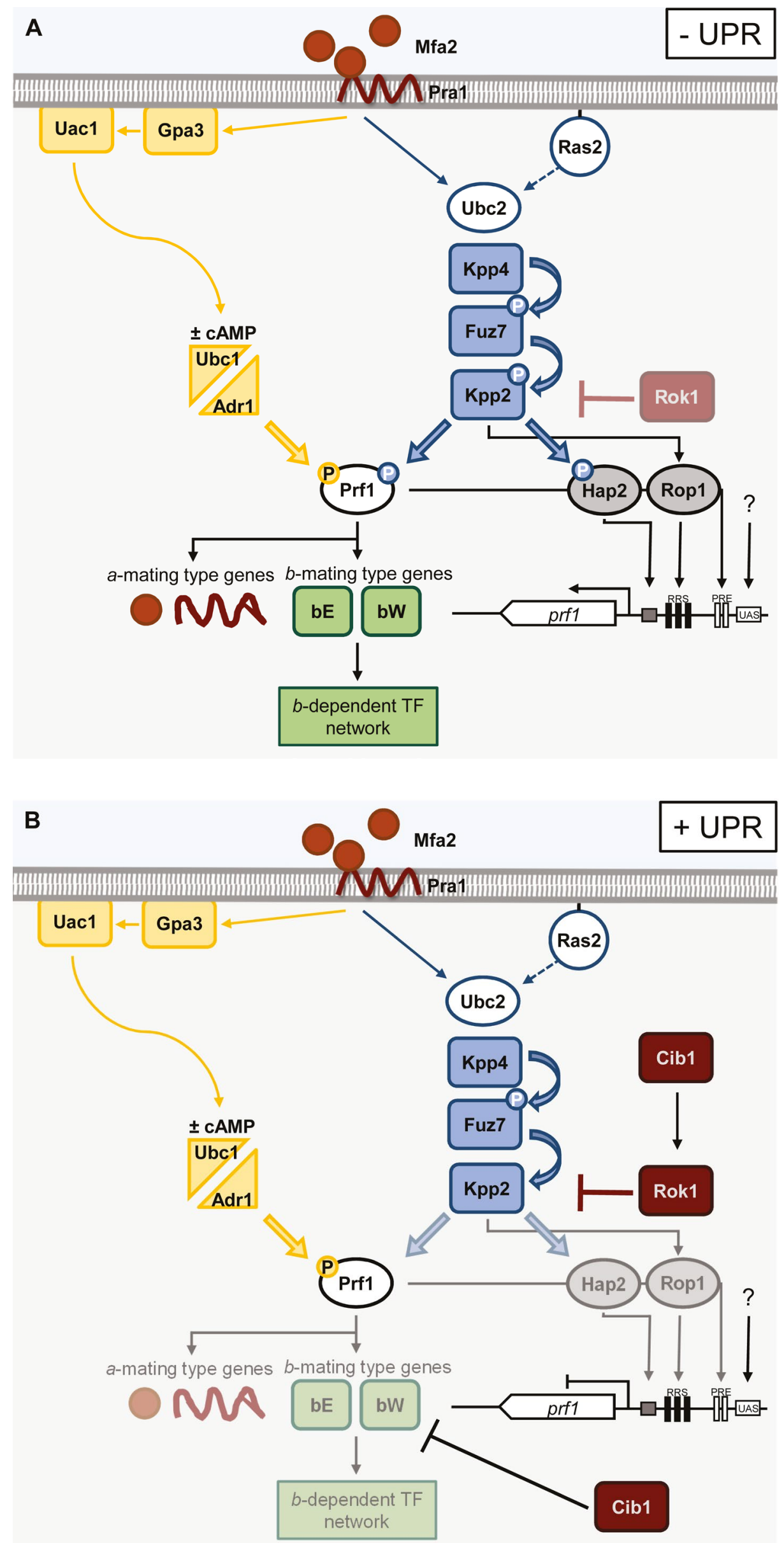
Figure 5.1 Model of the crosstalk between the UPR and mating type signaling pathways. (A) Pheromone sensing activates a MAPK (blue) and a PKA (yellow) signaling cascade, resulting in phosphorylation and increased expression of the central transcriptional regulator Prf1. This induces $a$ - and $b$-gene expression and $a$-mediated formation of conjugation tubes and mating. After fusion of two cells, the transcription factors bE and bW (green) form a heterodimer and regulate further pathogenic development. (B) After penetration of the plant surface, the UPR (dark red) is activated and remains active throughout pathogenic development in planta. An active UPR is required to suppress the plant defense response and to establish the biotrophic interaction. The UPR regulator Cib1 helps to trigger release of the cell cycle block and proliferation in planta. During further pathogenic development, crosstalk of the UPR and the DSP Rok1 constitutes a regulatory feedback mechanism that leads to inhibition of the $a$ - and $b$-dependent signaling pathways, thereby lowering effector gene expression and reducing ER-stress levels.

\subsection{A new approach for conditional gene expression}

\subsubsection{Promoters with conditional activity enable stage-specific gene expression}

Gene deletion or overexpression are common ways to characterize gene function. However, both methods only display an on/off situation and are thus limited with respect to the conclusions that can be drawn from these studies. Moreover, genes with essential cellular or developmental functions cannot be analyzed by conventional gene deletion approaches. Overexpression or induced expression on the other side, facilitated by constitutive promoters like the $U$. maydis otef promoter, or the nutrient-dependent crg (arabinoseinducible) and nar promoters (nitrate inducible) (Spellig et al., 1996; Brachmann et al., 2001), may affect cellular metabolism and result in pleiotropic effects. Therefore, systems that are metabolism independent and titratable have been established. The widespread tetracycline-based expression system has been adapted for U. maydis (Zarnack et al., 2006). This system is based on the E. coli TetR repressor that binds to the tet $O$ operator. Tetracycline binds TetR and prevents DNA binding, thereby gene expression is induced. In variations of the system, such as the Tet-Off system, TetR is fused to an activator domain and induces gene expression when bound to the tet $O$ element. Other inducible expression systems like the estrogen-induced system for Aspergillus sp. (Pachlinger et al., 2005) or the orzearalenone-induced system in Gibberella zeae (Lee et al., 2010) also require addition of an external compound to induce transcription. However, all these systems do not allow to manipulate gene expression during fungal/plant interactions.

Our approach aimed for a system that allows controlled/specific gene expression during plant infection. We used promoters that are either inactivated or activated after plant penetration enabling a conditional expression pattern for any given gene of interest. To obtain promoters facilitating a gene 'shut off', genes were chosen based on the following criteria: constitutive expression until 2 or 4 dpi, respectively, followed by a clear decrease 
in gene expression. Candidate promoters were identified based on recent RNAseq-based transcriptome analyses during various stages of biotrophic growth (Figure 5.2) (Lanver et al., 2018).

The previously established, plant-induced expression system based on the maize-induced (mig) genes was adapted for this purpose. The migl gene and the mig2 gene cluster are not expressed during yeast-like growth of U. maydis, but specifically induced in planta (Basse et al., 2000; Basse et al., 2002). However, the exact time of activation and expression strength differ within the genes (Figure 5.2). The parallel deletion of all mig genes did not affect pathogenic development of the fungus. The promoters of genes migl and mig2_1 were chosen due to the differences in their expression strength, facilitating high or average expression levels, respectively (Basse et al., 2000; Basse et al., 2002; Lanver et al., 2018).

Pilot experiments fusing the mig2_l promoter to the intronless $c i b 1^{\mathrm{s}}$ transcript followed by integration in the ip locus of $U$. maydis revealed de-regulation and high expression levels in axenic culture (see 5.2.2), suggesting that the genomic context is important for proper activity and regulation of the respective promoters.

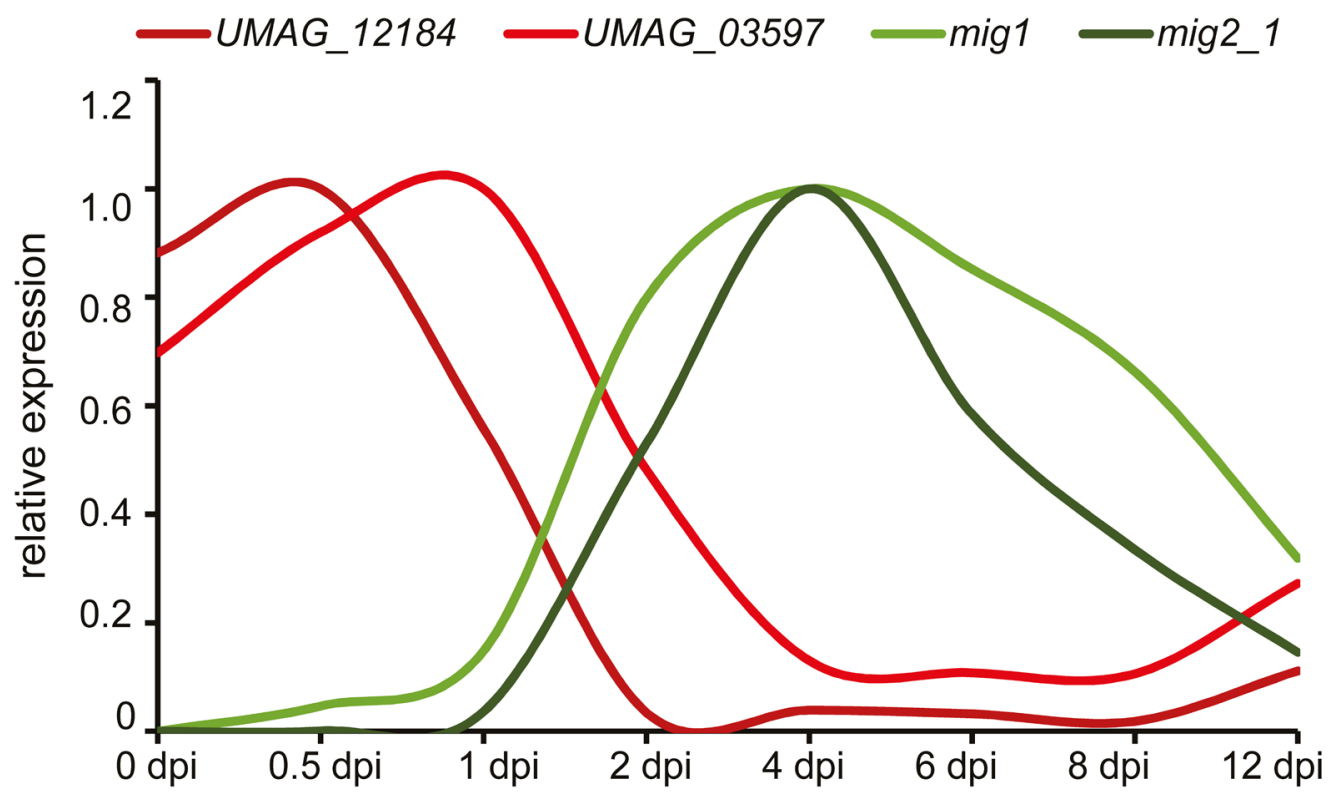

Figure 5.2 Expression pattern of conditionally expressed genes. Expression pattern of the $U M A G_{-}$12184, $U M A G \_03597, \operatorname{mig} 1$ (UMAG_03223) and mig2_1 (UMAG_06178) genes up to 12 days post inoculation (dpi). 6-day-old maize seedlings were injected with a mixture of compatible haploid strains FB1 and FB2 and plant material was harvested at the indicated time points. Normalized read counts of the genes were normalized to the exon length and the highest expression value for each gene was set to 1 . Raw data was extracted from Lanver et al., 2018 


\subsubsection{The locus of gene integration and the presence of resistance cassettes affect gene expression}

Since integration of expression constructs into the ip locus (Figure 5.3A) failed to produce the desired expression pattern, we tested if this was related to the genomic context. Indeed, only when the construct was integrated into the native genomic locus of the conditionally expressed genes and the resistance marker was removed by FLP/FRT recombination (Figure 5.3B), correct and conditional expression of $c i b 1^{\mathrm{s}}$ was achieved.

This effect is not specific for $c i b 1^{\mathrm{s}}$ since similar results were obtained for the pit $1 / 2$ genes. When integrated into the ip locus, expression of pit1 and pit2 (under control of their native promoter) was 400- and 800-fold higher than in WT cells, respectively. When integrated into the native pit1/2 locus, gene expression was still elevated, and only after removal of the resistance marker with the FLP/FRT recombinase system (Figure 5.3B) (Khrunyk et al., 2010), pit1/2 expression was restored to wild type-like levels.

pit1 and pit2 are virulence factors and part of a gene cluster that is, similar to the mig2 gene cluster, plant-specifically upregulated (Basse et al., 2002; Doehlemann et al., 2011). Position specific effects are potentially caused by alterations of the chromatin structure that might affect promoter activity and gene expression. Chromatin is a dynamic structure, and histone modifications like acetylation and methylation determine accessibility of DNA for a variety of factors including the transcription machinery (Seto and Yoshida, 2014; Freitag, 2017; Elías-Villalobos et al., 2019). Acetylation and deacetylation of histones affect their positive charge, thereby loosening or tightening the interaction between histone and DNA, respectively (Bannister and Kouzarides, 2011). In U. maydis, the histone deacetylases Hda1 is required for sporogenesis. Microarray analysis of $h d a l$ deletion strains showed a total of 21 regulated gene clusters (Treutlein, 2007). Interestingly, expression of the mig2 as well as of the pit1/2 gene cluster were induced in hdal deletion strains, suggesting that these gene clusters are subject to epigenetic regulation. Control of effector genes by influencing the chromatin structure appears to be a common feature of plant pathogenic fungi (Soyer et al., 2014).

Differences in gene expression when expression constructs were integrated into the native genomic were also related to the presence of the resistance cassette. Marker genes conferring resistance to a fungicide are normally under control of strong, constitutive promoters and could potentially also affect gene expression. Interestingly, in case of pit1/2, 
the choice of resistance cassette also affected expression level. The nourseothricin resistance cassette $\left(\mathrm{Nat}^{\mathrm{R}}\right)$ increased expression of pit1 and pit2 49- and 134-fold, respectively, whereas the hygromycin cassette increased expression 10- (pit1) and 13-fold (pit2). Hence, the application of conditional expression systems requires control experiments and the preservation of the genomic context.

A

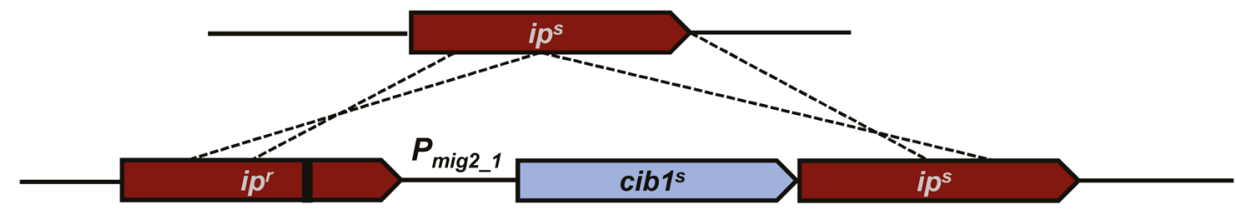

B
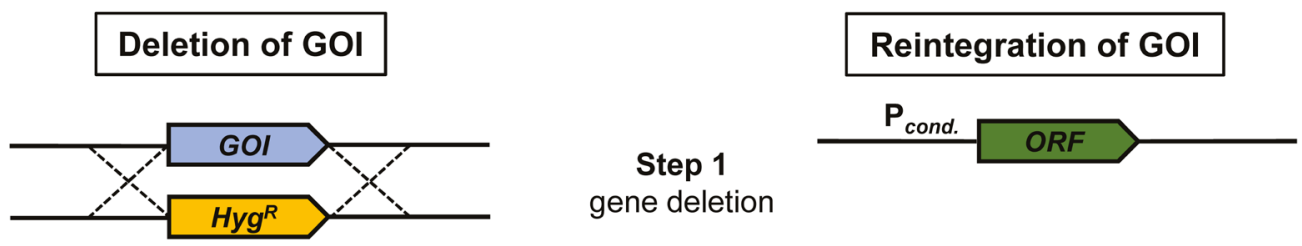

gene deletion
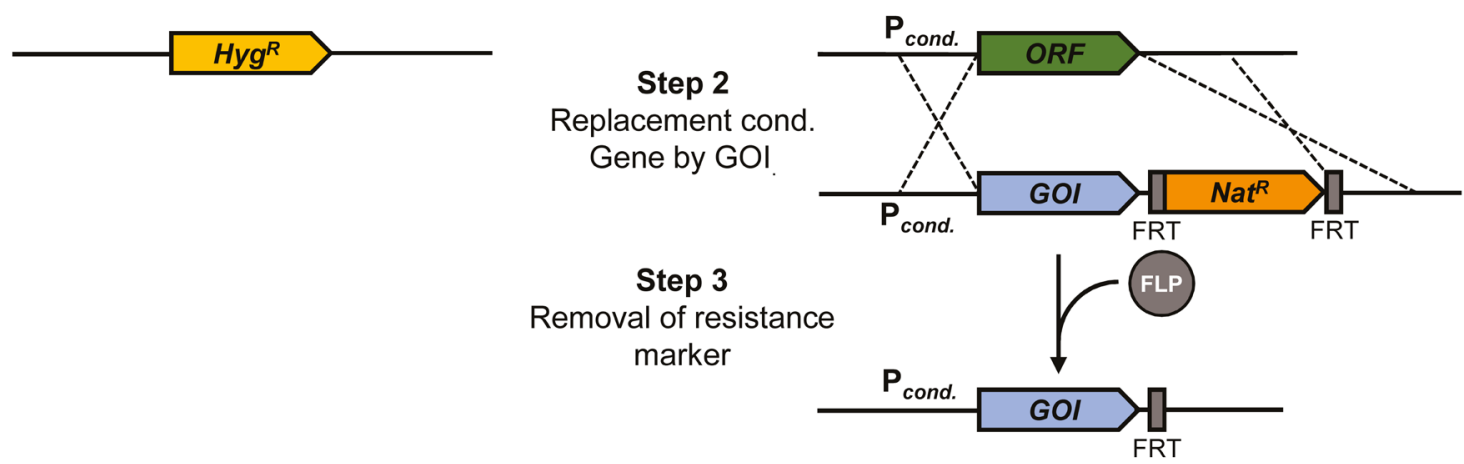

Figure 5.3 Cloning strategies for conditional gene expression. (A) Integration of $\mathrm{cibl}^{\mathrm{s}}$ under control of the mig2_l promoter into the $i p$ locus of $U$. maydis. (B) Integration of a gene of interest (GOI) into the locus of a conditionally expressed gene. 1) GOI is deleted from its native genomic locus. 2) The GOI is integrated into the genomic locus of the conditionally expressed gene, thereby replacing the native gene. 3) The resistance marker (here: $\mathrm{Nat}^{\mathrm{R}}$ ) is removed using the FLP/FRT recombination system.

\subsubsection{UPR activity is important at later stages of biotrophic development in planta}

It has been shown that correct timing of UPR activation is crucial for pathogenic development (Heimel et al., 2013). During early stages of pathogenic development, the unprocessed $c i b 1$ transcript is approximately 20 -fold more abundant than the spliced $c i b 1^{\mathrm{s}}$ transcript, the latter mediating Cib1 expression and UPR activity. After plant penetration, a large number of effectors is secreted to suppress the plant defense response and establish the biotrophic interaction. This results in increased ER-stress levels activating the UPR by 
unconventional splicing and increased expression of the cibl transcript. UPR activation stabilizes Clp1, thereby promoting the release of the cell cycle block and proliferation in planta. UPR activity is detectable throughout all stages of pathogenic development (Heimel et al., 2010b, Heimel et al., 2013), suggesting that the UPR is not only necessary to establish the biotrophic interaction, but also important for further pathogenic development in planta. Deletion of cibl leads to strongly attenuated virulence (Heimel et al., 2013) that was not suppressed by conditional expression of cibl using the $U M A G_{-} 12184$ promoter. By contrast, expression of cibl by the $U M A G \_03597$ promoter at least partially restored pathogenic development. This correlates with the extended activity of the UMAG_03597 promoter compared to the promoter of $U M A G_{-} 12184$ (Lanver et al., 2018). Additionally, microscopic analysis revealed that the strain expressing cibl under control of $\mathrm{P}_{U M A G_{-} 12184}$ formed filaments and appressoria on the plant surface, whereas proliferation in planta was blocked.

On the plant surface, U. maydis is recognized via pathogen-associated molecular patterns (PAMP) by the plant immune system, leading to transient upregulation of plant defense genes (Doehlemann et al., 2008). During establishment of the biotrophic interaction after plant penetration, these plant defense genes are suppressed in wild type strains. High $P R$-gene expression in strains expressing cibl under control of the $U M A G_{-} 12184$ promoter at 2 dpi indicate that the fungus was detected by the plant immune system on the plant surface and could not counteract the defense response. Consistent with previous data (Doehlemann et al., 2008), PRl is not induced during this early defense response. Thus, cibl gene expression under control of $\mathrm{P}_{U M A G_{-} 12184}$ was not sufficient to establish a compatible biotrophic interaction.

Strains expressing cibl under control of the $U M A G \_03597$ promoter were able to penetrate the plant surface, to induce hyphal branching and, to some extent, proliferation. Formation of clamp cells indicate release of the cell cycle block (Scherer et al., 2006). In comparison

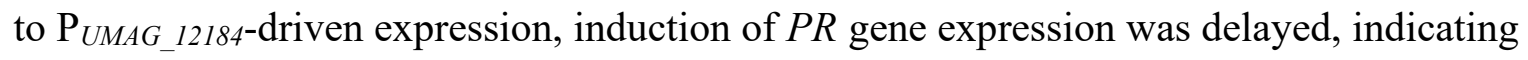
that the fungus was recognized by the plant immune system at later stages.

In $U$. maydis, deletion of $c i b 1$ leads to expression of the $P R 1, P R 3$ and $P R 5$ genes at 2 dpi (Heimel et al., 2013). This shows, together with our observations, that an active UPR is required for sustained suppression of the plant defense. Consistently, it has been observed that the UPR is important for efficient processing and secretion as well as for regulation of 
effectors (Lo Presti et al., 2015b; Hampel et al., 2016; Pinter et al., 2019). Cib1 binds to UPRE elements in the promoter of the pit1/2 genes, deletion of the UPRE element abolished expression of pit2 and significantly affected virulence (Hampel et al., 2016). Additionally, the signal peptide peptidase Spp1 has been identified as part of an UPR-regulated core gene set (Pinter et al., 2019). Spp1 is a key factor for fungal virulence and required to suppress the plant defense, deletion or mutation of the catalytic domain lead to a complete loss of virulence.

\subsubsection{Overexpression of cibl $^{\mathrm{s}}$ in planta does not lead to UPR hyperactivation}

After plant penetration, unconventional cytoplasmic splicing of the cibl transcript leads to expression of $c i b 1^{\mathrm{s}}$ and activation of the UPR. UPR activity is detected at all stages of pathogenic development in planta (Heimel et al., 2010a). Although premature expression of $c i b 1^{\mathrm{s}}$ negatively affected pathogenic development before plant infection, increased levels of $c i b 1^{\mathrm{s}}$ did not affect pathogenic growth in planta.

We used our conditional expression system to investigate if high-level expression of $c i b 1^{\mathrm{s}}$ results in UPR hyperactivation and disturbs the pathogenic development in planta. Interestingly, infection symptoms of $\mathrm{P}_{\text {mig2 }} 1: c i b 1^{\mathrm{s}}$ or $\mathrm{P}_{\text {migl }}: c i b 1^{\mathrm{s}}$ strains when used in combination with a compatible wild type strains were both similar to the WT control. This suggests, that robust control mechanisms efficiently prevent UPR hyperactivation by $c i b 1^{\text {s }}$.

One of these mechanisms is based on Clp1-dependent modulation of the UPR, conferring hyperresistance towards ER stress (Heimel et al., 2013). Interestingly, this correlates with reduced expression of conserved UPR marker genes including bip1, pdil, cne1, erol and cibl itself (Pinter et al., 2019). Moreover, strains lacking the unspliced cibl transcript were highly susceptible towards $c i b 1^{\text {s}}$-dependent UPR hyperactivation (Heimel et al., 2013). Thus, it is conceivable that in addition to Clp1, the unspliced cibl transcript (and thereby the $\mathrm{Cib} 1^{\mathrm{u}}$ protein) contributes to efficient pathogenic development during phases of high ER-stress/ high UPR activity. This idea is supported by the inability to express $c i b 1^{\mathrm{s}}$ under control of its native promoter in $\Delta c i b 1$ strains (Heimel et al., 2013). Under these conditions, the UPR is hyperactivated due to autoregulation of $c i b 1^{\mathrm{s}}$. In higher eukaryotes, it has been shown that $\mathrm{XBP} 1^{\mathrm{s}}$ and $\mathrm{XPB} 1^{\mathrm{u}}$, the spliced and unspliced version of the UPR regulator, can form homo- and heterodimers with $\mathrm{XBP} 1^{\mathrm{u}}$ acting as a negative regulator of $\mathrm{XBP} 1^{\mathrm{s}}$ (Yoshida et al., 2006). XBP $1^{\mathrm{u}}$ shuttles between the nucleus and the cytoplasm while XBP $1^{\mathrm{s}}$ acts in 
the nucleus and induces transcription of target genes. Interaction of XBP $1^{\mathrm{u}}$ and $\mathrm{XBP} 1^{\mathrm{s}}$ leads to nuclear export of the heterodimer and degradation via the proteasome (Tirosh et al., 2006; Yoshida et al., 2006). Consistently, $\mathrm{Cib} 1^{\mathrm{s}}$ and $\mathrm{Cib} 1^{\mathrm{u}}$ form homo- and heterodimers and overexpression of $\mathrm{Cib} 1^{\mathrm{u}}$ suppresses $\mathrm{Cib} 1^{\mathrm{s}}$-dependent gene regulation. Hence, it is conceivable that $\mathrm{Cib} 1^{\mathrm{u}}$ acts as a negative regulator to control $\mathrm{Cib} 1^{\mathrm{s}}$ activity (Hampel, 2016). Taken together, our results illustrate that a well-timed activation of the UPR is important for full virulence and efficient host colonization by $U$. maydis and that mechanisms to prevent UPR hyperactivation in planta are highly effective and most likely rely on the function of Clp1 and $\mathrm{Cib}^{\mathrm{u}}$ proteins. 


\section{References}

Andrews, D.L., Egan, J.D., Mayorga, M.E., and Gold, S.E. (2000) The Ustilago maydis $u b c 4$ and $u b c 5$ Genes Encode Members of a MAP Kinase Cascade Required for Filamentous Growth. Mol Plant-Microbe Interact 13: 781-786.

Bakkeren, G., Kämper, J., and Schirawski, J. (2008) Sex in smut fungi: Structure, function and evolution of mating-type complexes. Fungal Genet Biol 45: 15-21.

Bannister, A.J., and Kouzarides, T. (2011) Regulation of chromatin by histone modifications. Cell Res 21: 381-395.

Banuett, F. (1992) Ustilago maydis, the delightful blight. Trends Genet 8: 174-180.

Banuett, F., and Herskowitz, I. (1994) Identification of Fuz7, a Ustilago maydis MEK/MAPKK homolog required steps in the fungal life cycle. Genes Dev 8: 1367-1378.

Banuett, F., and Herskowitz, I. (1996) Discrete developmental stages during teliospore formation in the corn smut fungus, Ustilago maydis. Development 122: 2965-2976.

Banuett, F., and Herskowitz, I. (2006) Different a alleles of Ustilago maydis are necessary for maintenance of filamentous growth but not for meiosis. Proc Natl Acad Sci 86: 58785882.

Bardwell, L. (2006) Mechanisms of MAPK signalling specificity. Biochem Soc Trans 34: 837-841.

Bardwell, L., Martinez, A.R., Voora, D., Baggott, D.M., Thorner, J., and Cook, J.G. (2008) Repression of yeast Ste12 transcription factor by direct binding of unphosphorylated Kss1 MAPK and its regulation by the Ste7 MEK. Genes Dev 12: 2887-2898.

Basse, C.W., Kolb, S., and Kahmann, R. (2002) A maize-specifically expressed gene cluster in Ustilago maydis. Mol M 43: 75-93.

Basse, C.W., Stumpferl, S., and Kahmann, R. (2000) Characterization of a Ustilago maydis Gene Specifically Induced during the Biotrophic Phase: Evidence for Negative as Well as Positive Regulation. Mol Cell Proteomics 20: 329-339.

Bertolotti, A., Zhang, Y., Hendershot, L.M., Harding, H.P., and Ron, D. (2000) Dynamic interaction of BiP and ER stress transducers in the unfolded protein response. 2: 1-7. 
Blackwell, E., Halatek, I.M., Kim, H.N., Ellicott, A.T., Obukhov, A.A., and Stone, D.E. (2003) Effect of the Pheromone-Responsive $\mathrm{Ga}$ and Phosphatase Proteins of Saccharomyces cerevisiae on the Subcellular Localization of the Fus3 Mitogen-Activated Protein Kinase. Mol Cell Biol 23: 1135-1150.

Blackwell, E., Kim, H.-J.N., and Stone, D.E. (2007) The pheromone-induced nuclear accumulation of the Fus3 MAPK in yeast depends on its phosphorylation state and on Dig1 and Dig2. BMC Cell Biol 8: 1-15.

Bölker, M. (2001) Ustilago maydis - A valuable model system for the study of fungal dimorphism and virulence. Microbiology 147: 1395-1401.

Bölker, M., Genin, S., Lehmler, C., and Kahmann, R. (1995) Genetic regulation of mating and dimorphism in Ustilago maydis. Can J Bot 73: 320-325.

Bölker, M., Urban, M., and Kahmann, R. (1992) The a mating type locus of U. maydis specifies cell signaling components. Cell 68: 441-450.

Braakman, I., and Hebert, D. (2013) Protein Folding in the Endoplasmic Reticulum. Cold Spring Harb Perspect Biol 5:a013201.

Brachmann, A. (2001) Die frühe Infektionsphase von Ustilago maydis: Genregulation durch das bW/bE-Heterodimer. München, Ludwig-Maximilians-Universität. Doktorarbeit.

Brachmann, A., König, J., Julius, C., and Feldbrügge, M. (2004) A reverse genetic approach for generating gene replacement mutants in Ustilago maydis. Mol Genet Genomics 272: 216-226.

Brachmann, A., Schirawski, J., Müller, P., and Kahmann, R. (2003) An unusual MAP kinase is required for efficient penetration of the plant surface by Ustilago maydis. EMBO J 22: 2199-2210.

Brachmann, A., Weinzierl, G., Kämper, J., and Kahmann, R. (2001) Identification of genes in the bW/bE regulatory cascade in Ustilago maydis. Mol Microbiol 42: 1047-1063.

Brefort, T., Doehlemann, G., Mendoza-Mendoza, A., Reissmann, S., Djamei, A., and Kahmann, R. (2009) Ustilago maydis as a Pathogen. Annu Rev Phytopathol 47: 423-445.

Brefort, T., Müller, P., and Kahmann, R. (2005) The High-Mobility-Group Domain 
Transcription Factor Rop1 Is a Direct Regulator of prf1 in Ustilago maydis. Eukaryot Cell 4: 379-391.

Brondello, J.-M., Pouysségur, J., and McKenzie, F.R. (1999) Reduced MAP Kinase Phosphatase-1 Degradation After p42/p44MAPK-Dependent Phosphorylation. 286: 25142518.

Camps, M., Nichols, A., Gillieron, C., Antonsson, B., Muda, M., Chabert, C., et al. (1998) Catalytic Activation of the Phosphatase MKP-3 by ERK2 Mitogen-Activated Protein Kinase. Science (80- ) 280: 1262-1266.

Cao, W., Bao, C., Padalko, E., and Lowenstein, C.J. (2008) Acetylation of mitogenactivated protein kinase phosphatase-1 inhibits Toll-like receptor signaling. $J$ Exp Med 205: 1491-1503.

Cargnello, M., and Roux, P.P. (2011) Activation and Function of the MAPKs and Their Substrates, the MAPK-Activated Protein Kinases. Microbiol Mol Biol Rev 75: 50-83.

Cheon, S.A., Jung, K.W., Chen, Y.L., Heitman, J., Bahn, Y.S., and Kang, H.A. (2011) Unique evolution of the UPR pathway with a novel bZIP transcription factor, HxL1, for controlling pathogenicity of Cryptococcus neoformans. PLoS Pathog 7: 1-16.

Christensen, T.T. (1963) Corn Smut caused by Ustilago Maydis.

Cook, J., Bardwell, L., and Thorner, J. (1997) Inhibitory and activating functions for MAPK Kss1 in the S. cerevisiae filamentous-growth signalling pathway. Nature 390: 98101.

Cox, J.S., Chapman, R.E., and Walter, P. (1997) The unfolded protein response coordinates the production of endoplasmic reticulum protein and endoplasmic reticulum membrane. Mol Biol Cell 8: 1805-1814.

Cox, J.S., Shamu, C.E., and Walter, P. (1993) Transcriptional induction of genes encoding endoplasmic reticulum resident proteins requires a transmembrane protein kinase. Cell 73: $1197-1206$.

Cox, J.S., and Walter, P. (1996) A Novel Mechanism for Regulating Activity of a Transcription Factor That Controls the Unfolded Protein Response. Cell 87: 391-404.

Dard, N., and Peter, M. (2006) Scaffold proteins in MAP kinase signaling: more than 
simple passive activating platforms. BioEssays 28: 146-156.

Denu, J.M., Lohse, D.L., Vijayalakshmi, J., Saper, M.A., and Dixon, J.E. (1996) Visualization of intermediate and transition-state structures in protein-tyrosine phosphatase catalysis. Proc Natl Acad Sci 93: 2493-2498.

Djamei, A., and Kahmann, R. (2012) Ustilago maydis: Dissecting the Molecular Interface between Pathogen and Plant. PLoS Pathog 8: e1002955.

Dobson, C.M. (2003) Protein folding and misfolding. Nature 426: 884-890.

Doebley, J. (1992) Mapping the genes that made maize. Trends Genet 8: 302-307.

Doehlemann, G., Linde, K. van der, Aßmann, D., Schwammbach, D., Hof, A., Mohanty, A., et al. (2009) Pep1, a Secreted Effector Protein of Ustilago maydis, Is Required for Successful Invasion of Plant Cells. PLoS Pathog 5: 1-16.

Doehlemann, G., Reissmann, S., Aßmann, D., Fleckenstein, M., and Kahmann, R. (2011) Two linked genes encoding a secreted effector and a membrane protein are essential for Ustilago maydis-induced tumour formation. Mol Microbiol 81: 751-766.

Doehlemann, G., Wahl, R., Horst, R.J., Voll, L.M., Poree, F., Stitt, M., et al. (2008) Reprogramming a maize plant: transcriptional and metabolic changes induced by the fungal biotroph Ustilago maydis. Plant J 56: 181-195.

Doi, K., Gartner, A., Ammerer, G., Errede, B., Shinkawa, H., Sugimoto, K., and Matsumoto, K. (1994) MSG5, a novel protein phosphatase promotes adaptation to pheromone response in S. cerevisiae. EMBO J 13: 61-70.

Elías-Villalobos, A., Barrales, R.R., and Ibeas, J.I. (2019) Chromatin modification factors in plant pathogenic fungi: insights from Ustilago maydis. Fungal Genet Biol 129: 52-64.

Elion, E.A. (1995) SteS : a meeting place for MAP kinases and their associates. Trends Cell Biol 5: 3-8.

Fassio, A., and Sitia, R. (2002) Formation, isomerisation and reduction of disulphide bonds during protein quality control in the endoplasmic reticulum. Histochem Cell Biol 117: 151157.

Feldbrügge, M., Kämper, J., Steinberg, G., and Kahmann, R. (2004) Regulation of mating and pathogenic development in Ustilago maydis. Curr Opin Microbiol 7: 666-672. 
Feng, X., Krishnan, K., Richie, D.L., Aimanianda, V., Hartl, L., Grahl, N., et al. (2011) HacA-Independent functions of the ER stress sensor IreA Synergize with the canonical UPR to influence virulence traits in Aspergillus fumigatus. PLoS Pathog 7: 1-18.

Fjeld, C.C., Rice, A.E., Kim, Y., Gee, K.R., and Denu, J.M. (2000) Mechanistic basis for catalytic activation of mitogen-activated protein kinase phosphatase 3 by extracellular signal-regulated kinase. J Biol Chem 275: 6749-6757.

Flández, M., Cosano, I.C., Nombela, C., Martín, H., and Molina, M. (2004) Reciprocal Regulation between Slt2 MAPK and Isoforms of Msg5 Dual-specificity Protein Phosphatase Modulates the Yeast Cell Integrity Pathway. J Biol Chem 279: 11027-11034.

Flor-Parra, I., Vranes, M., Kämper, J., and Perez-Martin, J. (2006) Biz1, a Zinc Finger Protein Required for Plant Invasion by Ustilago maydis, Regulates the Levels of a Mitotic Cyclin. Plant Cell Online 18: 2369-2387.

Freitag, M. (2017) Histone Methylation by SET Domain Proteins in Fungi. Annu Rev Microbiol 71: 413-439.

García-Muse, T., Steinberg, G., and Pérez-Martín, J. (2003) Pheromone-Induced G2 Arrest in the Phytopathogenic Fungus Ustilago maydis . Eukaryot Cell 2: 494-500.

Garrido, E., and Pérez-Martín, J. (2003) The crk1 gene encodes an ime2-related protein that is required for morphogenesis in the plant pathogen Ustilago maydis. Mol Microbiol 47: $729-743$.

Garrido, E., Voß, U., Müller, P., Castillo-Lluva, S., Kahmann, R., and Pérez-Martín, J. (2004) The induction of sexual development and virulence in the smut fungus Ustilago maydis depends on Crk1, a novel MAPK protein. Genes Dev 7: 3117-3130.

Gillissen, B., Bergemann, J., Sandmann, C., Schroeer, B., Bölker, M., and Kahmann, R. (1992) A two-component regulatory system for self/non-self recognition in Ustilago maydis. Cell 68: 647-657.

Gold, S., Duncan, G., Barrett, K., and Kronstad, J. (1994) cAMP regulates morphogenesis in the fungal pathogen Ustilago maydis. Genes Dev 8: 2805-2816.

Gold, S.E., Brogdon, S.M., Mayorga, M.E., and Kronstad, J.W. (1997) The Ustilago maydis Regulatory Subunit of a cAMP-Dependent Protein Kinase Is Required for Gall Formation in Maize. Plant Cell 9: 1585. 
González-Rubio, G., Fernández-Acero, T., Martín, H.H., and Molina, M. (2019) MitogenActivated Protein Kinase Phosphatases (MKPs) in Fungal Signaling: Conservation, Function, and Regulation. Int J Mol Sci 20: 1709.

Good, M., Tang, G., Singleton, J., Reményi, A., and Lim, W.A. (2009) The Ste5 Scaffold Directs Mating Signaling by Catalytically Unlocking the Fus3 MAP Kinase for Activation. Cell 136: 1085-1097.

Good, M.C., Zalatan, J.G., and Lim, W.A. (2011) Scaffold proteins: Hubs for controlling the flow of cellular information. Science 332: 680-686.

Hampel, M. (2016) Analyse der UPR vermittelten Stressantwort und ihrer Funktion während der biotrophen Entwicklung von Ustilago maydis. Doktorarbeit.

Hampel, M., Jakobi, M., Schmitz, L., Meyer, U., Finkernagel, F., Doehlemann, G., and Heimel, K. (2016) Unfolded Protein Response (UPR) Regulator Cib1 Controls Expression of Genes Encoding Secreted Virulence Factors in Ustilago maydis. PLoS One 11: 1-16.

Hanson, S.R., Culyba, E.K., Hsu, T.-L., Wong, C.-H., Kelly, J.W., and Powers, E.T. (2009) The core trisaccharide of an N-linked glycoprotein intrinsically accelerates folding and enhances stability. Proc Natl Acad Sci 106: 3131-3136.

Harding, H.P., Zhang, Y., and Ron, D. (1999) Protein translation and folding are coupled by an resident kinase. Nature 398: 271-274.

Harding, H.P., Zhang, Y., Zeng, H., Novoa, I., Lu, P.D., Calfon, M., et al. (2003) An Integrated Stress Response Regulates Amino Acid Metabolism and Resistance to Oxidative Stress National Institute of Environmental Health Sciences. Mol Cell 11: 619-633.

Hartl, F.U., and Hayer-Hartl, M. (2009) Converging concepts of protein folding in vitro and in vivo. Nat Struct Mol Biol 16: 574-581.

Hartmann, H.A., Kahmann, R., and Bölker, M. (1996) The pheromone response factor coordinates filamentous growth and pathogenicity in Ustilago maydis. EMBO J 15: 16321641.

Hartmann, H.A., Kruger, J., Lottspeich, F., and Kahmann, R. (1999) Environmental Signals Controlling Sexual Development of the Corn Smut Fungus Ustilago maydis through the Transcriptional Regulator Prf1. Plant Cell 11: 1293-1305. 
Haze, K., Yoshida, H., Yanagi, H., Yura, T., and Mori, K. (1999) Mammalian Transcription Factor ATF6 Is Synthesized as a Transmembrane Protein and Activated by Proteolysis in Response to Endoplasmic Reticulum Stress. Mol Biol Cell 10: 3787-3799.

Heimel, K. (2015) Unfolded protein response in filamentous fungi - implications in biotechnology. Appl Microbiol Biotechnol 99: 121-132.

Heimel, K., Freitag, J., Hampel, M., Ast, J., Bölker, M., and Kamper, J. (2013) Crosstalk between the Unfolded Protein Response and Pathways That Regulate Pathogenic Development in Ustilago maydis. Plant Cell 25: 4262-4277.

Heimel, K., Scherer, M., Schuler, D., Kamper, J., Kämper, J., Heimel, K., et al. (2010a) The Ustilago maydis Clp1 Protein Orchestrates Pheromone and $b$-Dependent Signaling Pathways to Coordinate the Cell Cycle and Pathogenic Development. Plant Cell 22: 29082922.

Heimel, K., Scherer, M., Vranes, M., Wahl, R., Pothiratana, C., Schuler, D., et al. (2010b) The transcription factor Rbfl is the master regulator for $b$-mating type controlled pathogenic development in Ustilago maydis. PLoS Pathog 6: 1-13.

Heitman, J. (2015) Evolution of sexual reproduction: a view from the Fungal Kingdom supports an evolutionary epoch with sex before sexes. Fungal Biol Rev 118: 6072-6078.

Helenius, A. (2002) Intracellular Functions of N-Linked Glycans. Science 291: 2364-2369.

Hetz, C. (2012) The unfolded protein response: Controlling cell fate decisions under ER stress and beyond. Nat Rev Mol Cell Biol 13: 89-102.

Jacobs, D., Glossip, D., Xing, H., Muslin, A.J., and Kornfeld, K. (1999) Multiple docking sites on substrate proteins form a modular system that mediates recognition by ERK MAP kinase. Genes Dev 13: 163-175.

Joubert, A., Simoneau, P., Campion, C., Bataillé-Simoneau, N., Iacomi-Vasilescu, B., Poupard, P., et al. (2011) Impact of the unfolded protein response on the pathogenicity of the necrotrophic fungus Alternaria brassicicola. Mol Microbiol 79: 1305-1324.

Jung, K.-W., So, Y.-S., and Bahn, Y.-S. (2016) Unique roles of the unfolded protein response pathway in fungal development and differentiation. Sci Rep 6: 1-14.

Kaffarnik, F., Müller, P., Leibundgut, M., Kahmann, R., and Feldbrügge, M. (2003) PKA 
and MAPK phosphorylation of Prf1 allows promoter discrimination in Ustilago maydis. EMBO J 22: 5817-5826.

Kahmann, R., and Kämper, J. (2004) Ustilago maydis: How its biology relates to pathogenic development. New Phytol 164: 31-42.

Kamata, H., Honda, S.I., Maeda, S., Chang, L., Hirata, H., and Karin, M. (2005) Reactive oxygen species promote TNF $\alpha$-induced death and sustained JNK activation by inhibiting MAP kinase phosphatases. Cell 120: 649-661.

Kämper, J., Kahmann, R., Bölker, M., Ma, L.-J., Brefort, T., Saville, B.J., et al. (2006) Insights from the genome of the biotrophic fungal plant pathogen Ustilago maydis. Nature 444: 97-101.

Kämper, J., Reichmann, M., Romeis, T., Bölker, M., and Kahmann, R. (1995) Multiallelic recognition: Nonself-dependent dimerization of the $\mathrm{bE}$ and $\mathrm{bW}$ homeodomain proteins in ustilago maydis. Cell 81: 73-83.

Kawahara, T., Yanagi, H., Yura, T., and Mori, K. (1998) Unconventional Splicing of HAC1/ERN4 mRNA Required for the Unfolded Protein Reponse. Biochemistry 273: 18021807.

Khrunyk, Y., Münch, K., Schipper, K., Lupas, A.N., and Kahmann, R. (2010) The use of FLP-mediated recombination for the functional analysis of an effector gene family in the biotrophic smut fungus Ustilago maydis. New Phytol 957-968.

Kimmig, P., Diaz, M., Zheng, J., Williams, C.C., Lang, A., Aragón, T., et al. (2012) The unfolded protein response in fission yeast modulates stability of select mRNAs to maintain protein homeostasis. Elife 1: 1-20.

Klosterman, S.J., Martinez-Espinoza, A.D., Andrews, D.L., Seay, J.R., and Gold, S.E. (2008) Ubc2, an Ortholog of the Yeast Ste50p Adaptor, Possesses a BasidiomyceteSpecific Carboxy Terminal Extension Essential for Pathogenicity Independent of Pheromone Response. Mol Plant-Microbe Interact 21: 110-121.

Korennykh, A. V., Egea, P.F., Korostelev, A.A., Finer-Moore, J., Zhang, C., Shokat, K.M., et al. (2009) The unfolded protein response signals through high-order assembly of Ire1. Nature 457: 687-693.

Kronstad, J.W., and Leong, S.A. (1990) The $b$ mating-type locus of Ustilago maydis 
References

contains variable and constant regions. Genes Dev 4: 1384-1395.

Krüger, J., Loubradou, G., Regenfelder, E., Hartmann, A., and Kahmann, R. (1998) Crosstalk between cAMP and pheromone signalling pathways in Ustilago maydis. Mol Gen Genet 260: 193-198.

Kültz, D., and Burg, M. (1998) Evolution of osmotic stress signaling via MAP kinase cascades. J Exp Biol 201: 3015-21.

Lanver, D., Berndt, P., Tollot, M., Naik, V., Vranes, M., Warmann, T., et al. (2014) Plant Surface Cues Prime Ustilago maydis for Biotrophic Development. PLoS Pathog 10.

Lanver, D., Mendoza-Mendoza, A., Brachmann, A., and Kahmann, R. (2010) Sho1 and Msb2-Related Proteins Regulate Appressorium Development in the Smut Fungus Ustilago maydis. Plant Cell 22: 2085-2101.

Lanver, D., Müller, A.N., Happel, P., Schweizer, G., Haas, F.B., Franitza, M., et al. (2018) The Biotrophic Development of Ustilago maydis Studied by RNA-Seq Analysis. Plant Cell 30: $300-323$.

Lawan, A., Shi, H., Gatzke, F., and Bennett, A.M. (2013) Diversity and specificity of the mitrogen-acivated protein kinase phosphatase-1 functions. Cell Mol Life Sci 70: 223-237.

Lee, J., Son, H., Lee, S., Park, A.R., and Lee, Y.W. (2010) Development of a conditional gene expression system using a zearalenone-inducible promoter for the ascomycete fungus Gibberella zeae. Appl Environ Microbiol 76: 3089-3096.

Lee, N., and Kronstad, J.W. (2002) ras 2 Controls Morphogenesis, Pheromone Response, and Pathogenicity in the Fungal Pathogen Ustilago maydis. Eukaryot Cell 1: 954-966.

Lin, Y.-W., Chuang, S.-M., and Yng, J.-L. (2003) ERK1/2 Achieves Sustained Activation by Stimulating MAPK Phosphatase-1 Degradation via the Ubiquitin-Proteasome Pathway*. J Biol Chem 278: 21534-21541.

Lin, Y., and Yang, J. (2006) Cooperation of ERK and SCFSkp2 for MKP-1 Destruction Provides a Positive Feedback Regulation of Proliferating Signaling*. J Biol Chem 281: 915-926.

Liu, H., Styles, C.A., and Fink, G.R. (1993) Elements of the yeast pheromone response pathway required for filamentous growth of diploids. Science 262: 1741-1744. 
Ma, Y., Brewer, J.W., Alan Diehl, J., and Hendershot, L.M. (2002) Two distinct stress signaling pathways converge upon the CHOP promoter during the mammalian unfolded protein response. J Mol Biol 318: 1351-1365.

Madhani, H.D., Styles, C.A., and Fink, G.R. (1997) MAP kinases with Distinct Inhibitory Functions Impart Signaling Specificity during Yeast Differentiation. Cell 91: 673-684.

Mapes, J., and Ota, I.M. (2004) Nbp2 targets the Ptc1-type 2C Ser/Thr phosphatase to the HOG MAPK pathway. EMBO J 23: 302-311.

Mayorga, M.E., and Gold, S.E. (1998) Characterization and molecular genetic complementation of mutants affecting dimorphism in the fungus Ustilago maydis. Fungal Genet Biol 24: 364-376.

Mayorga, M.E., and Gold, S.E. (1999) A MAP kinase encoded by the $u b c 3$ gene of Ustilago maydis is required for filamentous growth and full virulence. Mol Microbiol 34: 485-497. Mayorga, M.E., and Gold, S.E. (2001) The $u b c 2$ gene of Ustilago maydis encodes a putative novel adaptor protein required for filamentous growth, pheromone response and virulence. Mol Microbiol 41: 1365-1379.

Mendoza-Mendoza, A., Berndt, P., Djamei, A., Weise, C., Linne, U., Marahiel, M., et al. (2009a) Physical-chemical plant-derived signals induce differentiation in Ustilago maydis. Mol Microbiol 71: 895-911.

Mendoza-Mendoza, A., Eskova, A., Weise, C., Czajkowski, R., and Kahmann, R. (2009b) Hap2 regulates the pheromone response transcription factor prf1 in Ustilago maydis. Mol Microbiol 72: 683-698.

Mitra, S., and Ryoo, H.D. (2019) The unfolded protein response in metazoan development. J Cell Sci 132: 1-9.

Miyazaki, T., Nakayama, H., Nagayoshi, Y., Kakeya, H., and Kohno, S. (2013) Dissection of Ire1 Functions Reveals Stress Response Mechanisms Uniquely Evolved in Candida glabrata. PLoS Pathog 9: 1-20.

Mori, K., Ma, W., Gething, M.J., and Sambrook, J. (1993) A Transmembrane Protein with a $\operatorname{cdc} 2^{+} / \mathrm{CDC} 28$-Related Kinase Activity Is Required for Signaling from the ER to the Nucleus. Cell 74: 743-756. 
Mori, K., Sant, A., Kohnol, K., Normington, K., Gething, M., and Sambrook, J.F. (1992) A $22 \mathrm{bp}$ cis-acting element is necessary and sufficient for the induction of the yeast KAR2 (BiP) gene by unfolded proteins. EMBO J 1: 2583-2593.

Morrow, C.A., and Fraser, J.A. (2009) Sexual reproduction and dimorphism in the pathogenic basidiomycetes. FEMS Yeast Res 9: 161-177.

Müller, O., Kahmann, R., Aguilar, G., Trejo-Aguilar, B., Wu, A., and Vries, R.P. de (2008) The secretome of the maize pathogen Ustilago maydis. Fungal Genet Biol 45: 63-70.

Müller, P. (2003) Signalweiterleitung in Ustilago maydis: kontrolliert Pheromonantwort und pathogene Entwicklung. Doktorarbeit.

Müller, P., Aichinger, C., Feldbrügge, M., and Kahmann, R. (1999) The MAP kinase Kpp2 regulates mating and pathogenic development in Ustilago maydis. Mol Microbiol 34: $1007-1017$.

Müller, P., Weinzierl, G., Brachmann, A., Feldbrügge, M., and Kahmann, R. (2003) Mating and Pathogenic Development of the Smut Fungus Ustilago maydis Are Regulated by One Mitogen-Activated Protein Kinase Cascade. Eukaryot Cell 2: 1187-1199.

Murray, J.I., Whitfield, M.L., Trinklein, N.D., Myers, R.M., Brown, P.O., and Botstein, D. (2004) Diverse and Specific Gene Expression Responses to Stresses in Cultured Human Cells. Mol Biol Cell 15: 2876-2889.

Nilsson, I.M., and Heijne, G. von (1993) Determination of the distance between the oligosaccharyltransferase active site and the endoplasmic reticulum membrane. $J$ Biol Chem 268: 5798-801.

Pachlinger, R., Mitterbauer, R., Adam, G., and Strauss, J. (2005) Metabolically independent and accurately adjustable Aspergillus sp. expression system. Appl Environ Microbiol 71: 672-678.

Patterson, K.I., Brummer, T., O’brien, P.M., and Daly, R.J. (2009) Dual-specificity phosphatases: critical regulators with diverse cellular targets. Biochem J 418: 475-489.

Pearson, G., Robinson, F., Gibson, T.B., Xu, B., Karandikar, M., Berman, K., and Cobb, M.H. (2001) Mitogen-Activated Protein (MAP) Kinase Pathways: Regulation and Physiological Functions. Endocr Rev 22: 153-183. 
Pinter, N., Hach, C.A., Hampel, M., Rekhter, D., Zienkiewicz, K., Feussner, I., et al. (2019) Signal peptide peptidase activity connects the unfolded protein response to plant defense suppression by Ustilago maydis. PLOS Pathog 15: 1-40.

Presti, L. Lo, López Díaz, C., Turrà, D., Pietro, A. Di, Hampel, M., Heimel, K., and Kahmann, R. (2015b) A conserved co-chaperone is required for virulence in fungal plant pathogens. New Phytol 208: 1-14.

Rechsteiner, M. (1996) PEST sequences and regulation by proteolysis. Trends Biochem Sci 21: $267-271$.

Richie, D.L., Hartl, L., Aimanianda, V., Winters, M.S., Fuller, K.K., Miley, M.D., et al. (2009) A role for the unfolded protein response (UPR) in virulence and antifungal susceptibility in Aspergillus fumigatus. PLoS Pathog 5: 1-17.

Robbins, D.J., Zhen, E., Owaki, H., Vanderbilt, C.A., Ebert, D., Geppert, T.D., and Cobb, M.H. (1993) Regulation and properties of extracellular signal-regulated protein kinases 1 and 2 in vitro. $J$ Biol Chem 268: 5097-5106.

Rogers, S., Wells, R., and Rechsteiner, M. (1986) Amino acid sequences common to rapidly degraded proteins: The PEST hypothesis. Science 234: 364-368.

Romeis, T., Brachmann, A., Kahmann, R., and Kämper, J. (2000) Identification of a target gene for the bE-bW homeodomain protein complex in Ustilago maydis. Mol Microbiol 37: $54-66$.

Ron, D., and Walter, P. (2007) Signal integration in the endoplasmic reticulum unfolded protein response. Nat Rev Mol Cell Biol 8: 519-529.

Rüegsegger, U., Leber, J.H., and Walter, P. (2001) Block of HAC1 mRNA translation by long-range base pairing is released by cytoplasmic splicing upon induction of the unfolded protein response. Cell 107: 103-114.

Ruggiano, A., Foresti, O., and Carvalho, P. (2014) ER-associated degradation: Protein quality control and beyond. J Cell Biol 204: 869-879.

Saito, H. (2010) Regulation of cross-talk in yeast MAPK signaling pathways. Curr Opin Microbiol 13: 677-683.

Sano, R., and Reed, J.C. (2013) ER stress-induced cell death mechnisms. Biochim Biophys 
Acta 34: 474-476.

Santo, R. Di, Aboulhouda, S., Weinberg, D.E., and Green, R. (2016) The fail-safe mechanism of post-transcriptional silencing of unspliced HAC1 mRNA. Elife 5: 1-23.

Saxena, M., and Mustelin, T. (2000) Extracellular signals and scores of phosphatases: All roads lead to MAP kinase. Semin Immunol 12: 387-396.

Scherer, M., Heimel, K., Starke, V., and Kämper, J. (2006) The Clp1 Protein Is Required for Clamp Formation and Pathogenic Development of Ustilago maydis. Plant Cell Online 18: $2388-2401$.

Schmitz, L., McCotter, S., Kretschmer, M., Kronstad, J.W., and Heimel, K. (2018) Transcripts and tumors: regulatory and metabolic programming during biotrophic phytopathogenesis. F1000 Res 7: 1-8.

Schröder, M., Chang, J.S., and Kaufman, R.J. (2000) The unfolded protein response represses nitrogen-starvation induced developmental differentiation in yeast. Genes Dev 14: $2962-2975$.

Schröder, M., Clark, R., Liu, C.Y., and Kaufman, R.J. (2004) The unfolded protein response represses differentiation through the RPD3-SIN3 histone deacetylase. EMBO J 23: 2281-2292.

Schuck, S., Prinz, W.A., Thorn, K.S., Voss, C., and Walter, P. (2009) Membrane expansion alleviates endoplasmic reticulum stress independently of the unfolded protein response. $J$ Cell Biol 187: 525-536.

Schulz, B., Banuett, F., Dahl, M., Schlesinger, R., Schäfer, W., Martin, T., et al. (1990) The $b$ alleles of $U$. maydis, whose combinations program pathogenic development, code for polypeptides containing a homeodomain-related motif. Cell 60: 295-306.

Scrimale, T., Didone, L., Mesy Bentley, K.L. de, and Krysan, D.J. (2009) The Unfolded Protein Reponse Is Induced by the Cell Wall Integrity Mitogen-activated Protein Kinase Signaling Cascade and Is Required for Cell Wall Integrity in Saccharomyces cerevisiae. Seikagaku 20: 164-175.

Sellam, A., Dongo, A., Guillemette, T., Hudhomme, P., and Simoneau, P. (2007) Transcriptional responses to exposure to the brassicaceous defence metabolites camalexin and allyl-isothiocyanate in the necrotrophic fungus Alternaria brassicicola. Mol Plant 
Pathol 8: 195-208.

Seto, E., and Yoshida, M. (2014) Erasers of histone acetylation: The histone deacetylase enzymes. Cold Spring Harb Perspect Biol 6.

Shamu, C.E., and Walter, P. (1996) Oligomerization and phosphorylation of the Ire1p kinase during intracellular signaling from the endoplasmic reticulum to the nucleus. $E M B O$ J 15: 3028-39.

Shore, G.C., Papa, F.R., and Oakes, S.A. (2011) Signaling Cell Death from the Endoplasmic Reticulum Stress Response. Curr Opin Cell Biol 34: 474-476.

Siberstein, S., and Gilmore, R. (1996) Biochemistry, molecular biology, and genetics of the oligosaccharyltransferase. Faseb $J$ 10: 849-858.

Sidrauski, C., and Walter, P. (1997) The transmembrane kinase Irelp is a site-specific endonuclease that initiates mRNA splicing in the unfolded protein response. Cell 90: 10311039.

Skibbe, S.D., Doehlemann, G., Fernandes, J., and Walbot, V. (2010) Maize Tumors Caused by Ustilago Genes in Host and Pathogen. Science (80-) 67: 89-93.

Smith, M.H., Ploegh, H.L., and Weissman, J.S. (2011) Road to ruin: Targeting proteins for degradation in the endoplasmic reticulum. Science 334: 1086-1090.

Snetselaar, K.M., Bölker, M., and Kahmann, R. (1996) Ustilago maydis mating hyphae orient their growth toward pheromone sources. Fungal Genet Biol 20: 299-312.

Snetselaar, K.M., and Mims, C.W. (1992) Sporidial Fusion and Infection of Maize Seedlings by the Smut Fungus Ustilago Maydis. Mycologia 84: 193-203.

Snetselaar, K.M., and Mims, C.W. (1993) Infection of Maize Stigmas by Ustilago maydis: Light and Electron Microscopy. Phytopathology 83: 843-850.

Soyer, J.L., Ghalid, M. El, Glaser, N., Ollivier, B., Linglin, J., Grandaubert, J., et al. (2014) Epigenetic Control of Effector Gene Expression in the Plant Pathogenic Fungus Leptosphaeria maculans. PLoS Genet 10: 1-19.

Spellig, T., Bölker, M., Lottspeich, F., Frank, R.W., and Kahmann, R. (1994) Pheromones trigger filamentous growth in Ustilago maydis. EMBO J 13: 1620-1627.

Spellig, T., Bottin, A., and Kahmann, R. (1996) Green fluorescent protein (GFP) as a new 
References

vital marker in the phytopathogenic fungus Ustilago maydis. Mol Gen Genet 252: 503509.

Stasio, M. Di (2009) Regulation des Kpp2/Kpp6-MAPK-Moduls in Ustilago maydis durch Phosphatasen. Doktorarbeit.

Stasio, M. Di, Brefort, T., Mendoza-Mendoza, A., Münch, K., and Kahmann, R. (2009) The dual specificity phosphatase Rok1 negatively regulates mating and pathogenicity in Ustilago maydis. Mol Microbiol 73: 73-88.

Steinberg, G., and Perez-Martin, J. (2008) Ustilago maydis, a new fungal model system for cell biology. Trends Cell Biol 18: 61-67.

Tabas, I., and Ron, D. (2011) Effects of nanomechanical bone tissue properties on bone tissue strain: Implications for osteocyte mechanotransduction. J Musculoskelet Neuronal Interact 8: $330-331$.

Tang, W., Yanyan, R., Hong, L., Zhu, Q., Zuo, R., Guo, X., et al. (2015) System-wide characterization of bZIP transcription factor proteins involved in infection-related morphogenesis of Magnaporthe oryzae. Env Microbiol 17: 1377-1396.

Tirosh, B., Iwakoshi, N.N., Glimcher, L.H., and Ploegh, H.L. (2006) Rapid turnover of unspliced Xbp-1 as a factor that modulates the unfolded protein response. J Biol Chem 281: $5852-5860$.

Travers, K.J., Lockhart, D.J., Wodicka, L., Patil, C.K., Walter, P., and Weissman, J.S. (2000) Functional and Genomic Analyses Reveal an Essential Coordination between the Unfolded Protein Response and ER-Associated Degradation. Cell 101: 249-258.

Treutlein, M. (2007) Funktionelle Charakterisierung des Ruml-Proteins aus dem phytopathogenen Pilz Ustilago maydis. Doktorarbeit.

Urban, M., Kahmann, R., and Bölker, M. (1996) Identification of the pheromone response element in Ustilago maydis. Mol Gen Genet 251: 31-37.

Wahl, R., Zahiri, A., and Kämper, J. (2010) The Ustilago maydis b mating type locus controls hyphal proliferation and expression of secreted virulence factors in planta. Mol Microbiol 75: 208-220.

Wallis, A.K., and Freedman, R.B. (2011) Assisting Oxidative Protein Folding: How Do 
Protein Disulphide-Isomerases Couple Conformational and Chemical Processes in Protein Folding? In Molecular Chaperones. Jackson, S. (ed.). Springer Berlin Heidelberg, Berlin, Heidelberg. 1-34.

Walter, P., and Gardner, B.M. (2011) Unfolded Proteins Are Ire1-Activating Ligands That Directly Induce the Unfolded Protein Response. Science 333: 1891-1894.

Walter, P., and Ron, D. (2011) The Unfolded Protein Reponse: From Stress Pathway to Homeostatic Regulation. Science 334: 1081-1086.

Wang, R.-J., Peng, J., Li, Q.X., and Peng, Y.-L. (2017) Phosphorylation-mediated Regulatory Networks in Mycelia of Pyricularia oryzae Revealed by Phosphoproteomic Analyses. Mol Cell Proteomics 16: 1669-1682.

Widmann, C., Gibson, S., Jarpe, M.B., and Johnson, G.L. (1999) Mitogen-Activated Protein Kinase: Conservation of a Three-Kinase Module From Yeast to Human. Physiol Rev 79: 143-180.

Willoughby, E.A., Perkins, G.R., Collins, M.K., and Whitmarsh, A.J. (2003) The JNKinteracting protein-1 scaffold protein targets MAPK phosphatase-7 to dephosphorylate JNK. J Biol Chem 278: 10731-10736.

Yoshida, H., Matsui, T., Yamamoto, A., Okada, T., and Mori, K. (2001) XBP1 mRNA is induced by ATF6 and spliced by IRE1 in response to ER stress to produce a highly active transcription factor. Cell 107: 881-891.

Yoshida, H., Oku, M., Suzuki, M., and Mori, K. (2006) pXBP1(U) encoded in XBP1 premRNA negatively regulates unfolded protein response activator $\mathrm{pXBP} 1(\mathrm{~S})$ in mammalian ER stress response. J Cell Biol 172: 565-575.

Yoshida, H., Uemura, A., and Mori, K. (2009) pXBP1(U), a Negative Regulator of the Unfolded Protein Response Activator pXBP1(S), Targets ATF6 but not ATF4 in Proteasome-mediated Degradation. Cell Struct Funct 34: 1-10.

Zarnack, K., Eichhorn, H., Kahmann, R., and Feldbrügge, M. (2008) Pheromone-regulated target genes respond differentially to MAPK phosphorylation of transcription factor Prfl. Mol Microbiol 69: 1041-1053.

Zarnack, K., Maurer, S., Kaffarnik, F., Ladendorf, O., Brachmann, A., Kämper, J., and Feldbrügge, M. (2006) Tetracycline-regulated gene expression in the pathogen Ustilago 
References

maydis. Fungal Genet Biol 43: 727-738.

Zhan, X.-L., Deschenes, R.J., and Guan, K.-L. (1997) Differential regulation of FUS3 MAP kinase by tyrosine-specific phosphatases PTP2/PTP3 and dual-specificity phospatase MSG5. Genes Dev 11: 1690-1702.

Zhou, Z., Gartner, A., Cade, R., Ammerer, G., and Errede, B. (1993) Pheromone-induced signal transduction in Saccharomyces cerevisiae requires the sequential function of three protein kinases. Mol Cell Biol 13: 2069-80.

Zinszner, H., Kuroda, M., Wang, X.Z., Batchvarova, N., Lightfoot, R.T., Remotti, H., et al. (1998) CHOP is implicated in programmed cell death in response to impaired function of the endoplasmic reticulum. Genes Dev 12: 982-9 